\title{
Identification of Regions Responsible for the Open Conformation of S100A10 Using Chimaeric S100A11/S100A10 Proteins
}

\author{
Liliana Santamaria-Kisiel, The University of Western Ontario \\ Supervisor: Dr. Gary Shaw, The University of Western Ontario \\ A thesis submitted in partial fulfillment of the requirements for the Doctor of Philosophy degree \\ in Biochemistry \\ (C) Liliana Santamaria-Kisiel 2010
}

Follow this and additional works at: https://ir.lib.uwo.ca/etd

Part of the Biochemistry Commons, Molecular Biology Commons, Other Biochemistry, Biophysics, and Structural Biology Commons, and the Structural Biology Commons

\section{Recommended Citation}

Santamaria-Kisiel, Liliana, "Identification of Regions Responsible for the Open Conformation of S100A10 Using Chimaeric S100A11/S100A10 Proteins" (2010). Electronic Thesis and Dissertation Repository. 105. https://ir.lib.uwo.ca/etd/105

This Dissertation/Thesis is brought to you for free and open access by Scholarship@Western. It has been accepted for inclusion in Electronic Thesis and Dissertation Repository by an authorized administrator of Scholarship@Western. For more information, please contact wlswadmin@uwo.ca. 


\title{
IDENTIFICATION OF REGIONS RESPONSIBLE FOR THE OPEN CONFORMATION OF S100A10 USING CHIMAERIC S100A11/S100A10 PROTEINS
}

\author{
(Spine title: Chimaeric S100A11/S100A10 proteins) \\ (Thesis format: Integrated-Article)
}

by

\author{
Liliana Santamaria-Kisiel
}

Graduate Program in Biochemistry

\begin{abstract}
A thesis submitted in partial fulfilment
of the requirements for the degree of

Doctor of Philosophy
\end{abstract}

The School of Graduate and Postdoctoral Studies

The University of Western Ontario

London, Ontario, Canada

(C) Liliana Santamaria-Kisiel 2011 
THE UNIVERSITY OF WESTERN ONTARIO

SCHOOL OF GRADUATE AND POSTDOCTORAL STUDIES

CERTIFICATE OF EXAMINATION

$\underline{\text { Supervisor }}$

Examiners

Dr. Gary Shaw

Dr. Wing-Yiu-Choy

Dr. Eric Ball

Dr. Dale Laird

Dr. Walter Chazin

The thesis by

\title{
Liliana Santamaria-Kisiel
}

entitled:

\section{Identification Of Regions Responsible For The Open Conformation Of S100A10 Using Chimaeric S100A11/S100A10 Proteins}

\author{
is accepted in partial fulfilment of the \\ requirements for the degree of \\ Doctor of Philosophy
}

Date 


\begin{abstract}
S100A11 is a dimeric, EF-hand calcium-binding protein. Calcium binding to S100A11 results in a large conformational change that uncovers a broad hydrophobic surface used to interact with phospholipid-binding proteins (annexins A1 and A2), and facilitate membrane vesiculation events. In contrast to other S100 proteins, S100A10 is unable to bind calcium due to deletion and substitution of calcium-ligating residues. Despite this, calcium-free S100A10 assumes an "open" conformation that is very similar to S100A11 in its calcium-bound state $\left(\mathrm{Ca}^{2+}-\mathrm{S} 100 \mathrm{~A} 11\right)$. To understand how S100A10 is able to adopt an open conformation in the absence of calcium, seven chimaeric proteins were constructed where regions from calcium binding sites I and II, and helices II-IV in S100A11 were replaced with the corresponding regions of S100A10. The chimaeric proteins having substitutions in calcium-binding site II displayed increased hydrophobic surface exposure as assessed by ANS fluorescence and phenyl Sepharose binding in the absence of calcium. This response is similar to that observed for $\mathrm{Ca}^{2+}-\mathrm{S} 100 \mathrm{~A} 11$ and calcium-free S100A10. Further, this substitution resulted in calcium-insensitive binding to annexin A2 for one chimaeric protein. The results indicate that residues within site II are important in stabilizing the open conformation of S100A10 and presentation of its target-binding site. In contrast, S100A11 chimaeric proteins with helical substitutions displayed poorer hydrophobic surface exposure and consequently, unobservable annexin A2 binding. This work represents a first attempt to systematically understand the molecular basis for the calcium-insensitive open conformation of S100A10.
\end{abstract}


Keywords: EF-hand, calcium-binding protein, hydrophobicity, conformational change,

fluorescence, NMR spectroscopy, Protein design. 


\section{CO-AUTHORSHIP}

\section{Chapter 1:}

A version of this chapter has been published. Reproduced with permission from Santamaria-Kisiel, L., Rintala-Dempsey, A.C. and Shaw, G.S. 2006. Calcium-dependent and independent- interactions of the S100 protein family. Biochem J 396, 201-214. The manuscript was written by all three authors and final revisions were made by Dr. G.S. Shaw.

\section{Chapter 2 and 3}

Sections of these chapters are excerpt from the published manuscript SantamariaKisiel, L. and Shaw, G.S. (2011). Identification of Regions Responsible for the Open Conformation of S100A10 using Chimaeric S100A11-S100A10 Proteins. Biochem J. $434,37-48$. I wrote the first draft of the manuscript and made a number of revisions based on recommendations made by Dr. G.S. Shaw

\section{Chapter 4}

This chapter contains an expanded version of the published manuscript Santamaria-Kisiel, L. and Shaw, G.S. (2011). Identification of Regions Responsible for the Open Conformation of S100A10 using Chimaeric S100A11-S100A10 Proteins. Biochem J. 434, 37-48. Additional experiments, figures and written portions of the chapter were generated by L. Santamaria-Kisiel. 


\section{Chapter 5}

This chapters contains parts from the published manuscript Rintala-Dempsey, A.C., Santamaria-Kisiel, L., Liao, Y., Lajoie, G., Shaw, G.S. (2006). Insights into S100 target specificity examined by a new interaction between S100A11 and annexin A2. Biochemistry 45(49): 14695-705. Y.Liao (Dr. G. Lajoie laboratory. University of Western Ontario) synthesized and purified annexin A2 peptide. A.C. Rintala-Dempsey carried out the peptide array experiments and generated Figure 5. L. Santamaria-Kisiel conducted the remaining experiments, wrote the additional sections of the chapter and generated the figures. 


\section{DEDICATION}

A las dos mujeres de mi vida.

La que me enseñó a siempre seguir mis sueños: $\mathcal{M}$ i Mami

La que me enseña a soñar cada dia: Mi Hija adorada Isabella

To two special women in my life

The one who taught me to always follow my dreams: My Mom

The one who teaches me to dream everyday: My beloved daughter Isabella 


\section{ACKNOWLEDGEMENTS}

First and foremost, I would like to thank my supervisor Dr. Gary Shaw for his encouragement, his support and guidance. I could have not asked for a better supervisor. He has been more than a mentor; he had taught me lessons beyond science that would last forever. Thank you Gary!

To my advisory committee Dr. Hong Ling and Dr. Eric Ball for useful discussions regarding the project.

To Dr. Stan Dunn, for the opportunity of working in the BICF as a Facility Manager while completing my Ph.D. I really enjoy it.

To Dr. Walter Chazin (Vanderbilt University) for critical discussions and valuable insights in this project.

Thanks to Dr. Moises Wassermman, in Colombia, for introducing me to research and science, for giving me the foundation to be a good scientist.

Thanks to Kathy for all her help through the years. From numerous proofreads to making baby blankets. You are a wonderful person and I truly enjoyed working with you. I am going to miss you.

I have no words to thank my friends Paola, Diana and my mother in-law Lila for all the support and encouragement during the Grad school years, specially the last few months.

Thanks to the members of the Shaw lab for all the memories.

To Barb, Melita and Debbie in the Biochemistry office. Barb you are amazing and your help has been invaluable. 
A big THANK YOU to Don, Brian, Kathy and Anne for their tremendous assistance during my last stages of writing. Your encouragement, your support and your help made it all happen. Thank you all!

To the best part of grad school, my friends Heidi and Sue for keeping me right on track.

Thanks to my family back home in Colombia, for all the love and support despite the distance. I love you so much and I can't wait to go back and be together again. To my mom for being a great friend, and teacher. For her love, support and encouragement.

To my husband, my TKO, for staying by my side. Tomasz if we can survive grad school we can survive anything.

And at last, but not least, many many many thanks to the most important person in my life: Isabella. You, my little princess have changed my life, you make anything possible. I absolutely adore you. Nothing better than coming back home after a long day of endless writing, to see you smile and ask me why I have pictures of hair ribbons drawn on my papers while looking at protein structures. You are simply the best! 


\section{TABLE OF CONTENTS}

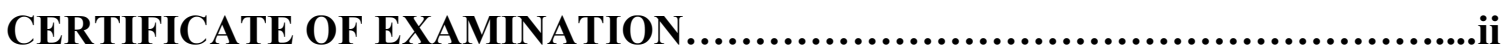

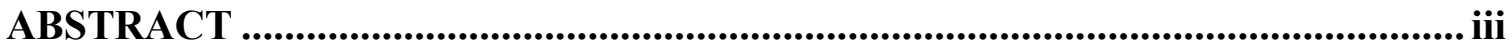

CO-AUTHORSHIP ................................................................................................ v

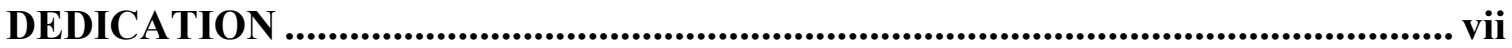

ACKNOWLEDGEMENTS ............................................................................... viii

TABLE OF CONTENTS ......................................................................................... $\mathrm{x}$

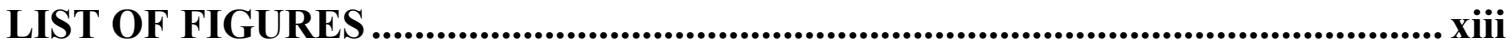

LIST OF TABLES .............................................................................................. xvi

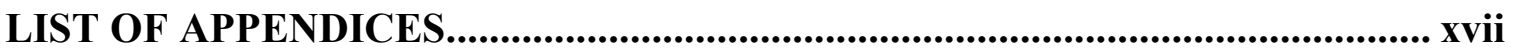

LIST OF ABBREVIATIONS, SYMBOLS AND NOMENCLATURE ................ xviii

Chapter 1 INTRODUCTION ............................................................................... 1

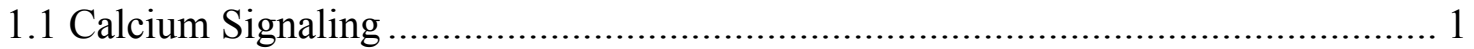

1.2 EF-hand motif ........................................................................................ 3

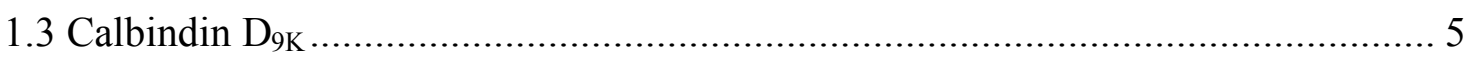

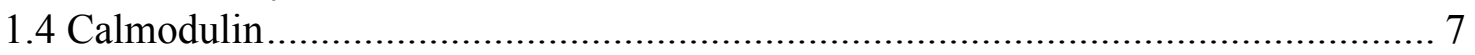

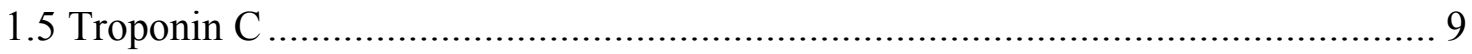

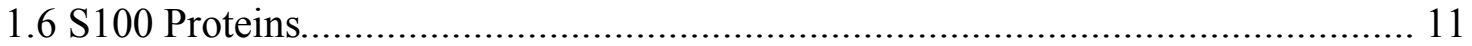

1.6.1 Structures of S100 proteins ............................................................... 14

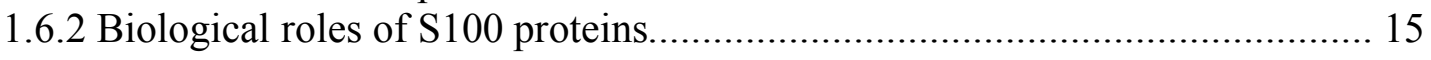

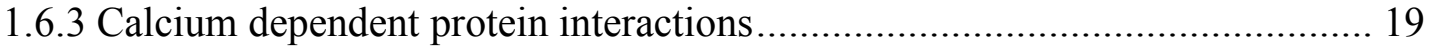

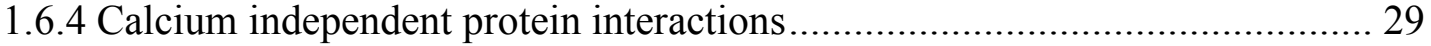

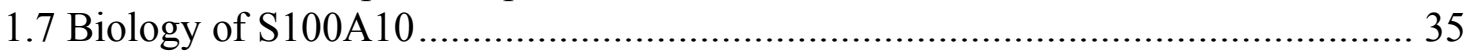

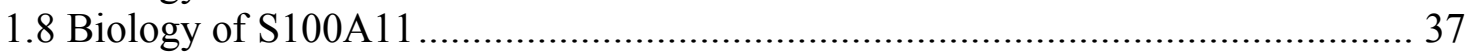

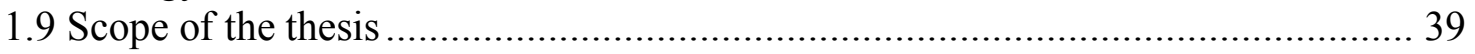

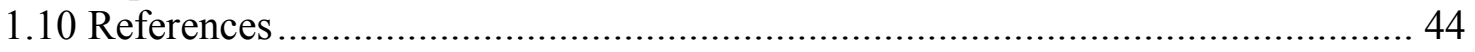

\section{Chapter 2 COMPARATIVE ANALYSIS OF S100A11 AND}

S100A10 PROTEINS...................................................................... 56

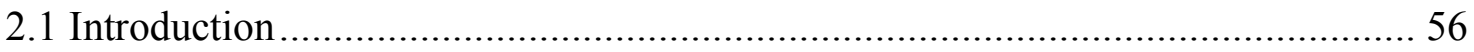

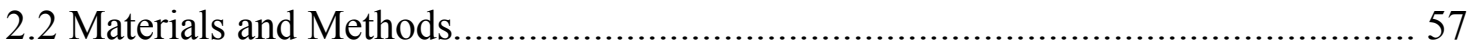

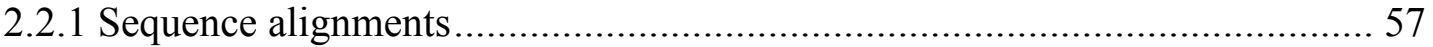

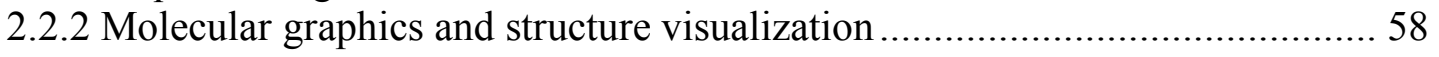

2.2.3 Interhelical angle measurements.................................................... 59

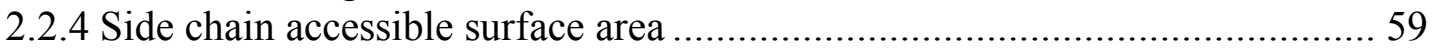

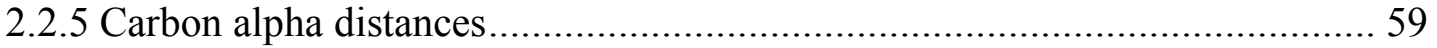

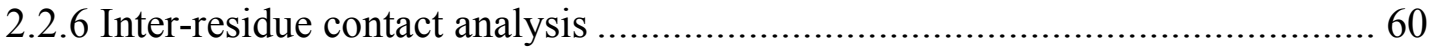

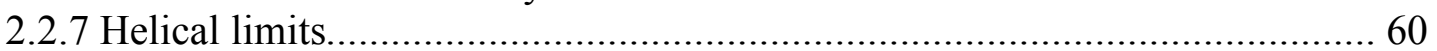

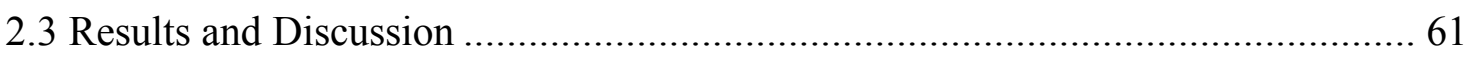

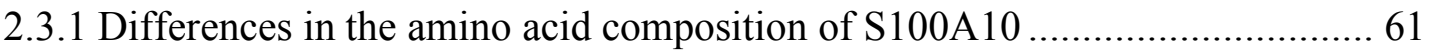

2.3.1.1 Sequence similarity between S100A10 and S100A11 ..........................6 65

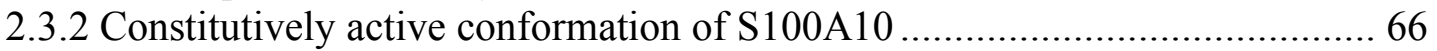


2.3.2.1 Interhelical packing …....................................................................... 72

2.3.2.2 Surfaces of S100A11 and S100 proteins ............................................... 77

2.3.3 Structural effects of substitutions in S100A10 flexible regions..................... 84

2.3.3.1 Shortening of the N-terminal loop in S100A10 affects backbone folding 86

2.3.3.2 "Calcium-ready" folding of loop II in S100A10 ……............................. 89

2.3.3.3 Characteristics of the linker region in the open form of S100A10............ 93

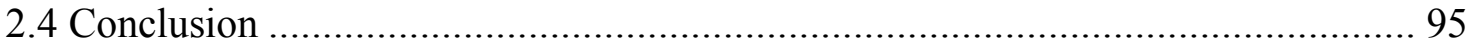

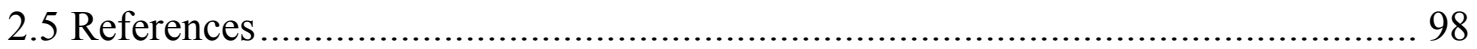

\section{Chapter 3 DESIGN, SYNTHESIS AND CHARACTERIZATION OF} CHIMAERIC S100A11/S100A10 PROTEINS ....................................101

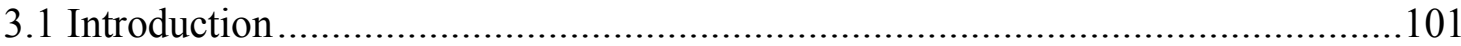

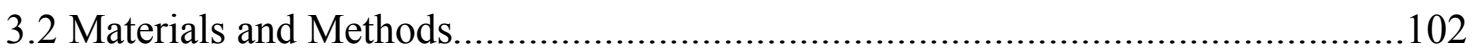

3.2.1 Cloning of Chimaeric S100A11/S100A10 proteins ……..............................102

3.2.2 Protein Expression and Purification.............................................................104

3.2.3 Oligomeric nature of chimaeric S100A11 proteins ......................................107

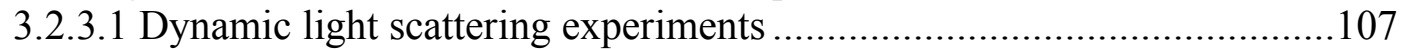

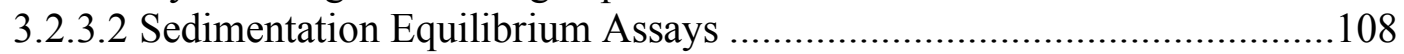

3.2.4 Characterization of chimaeric S100A11 proteins ........................................109

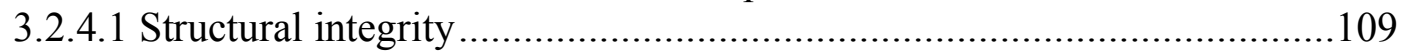

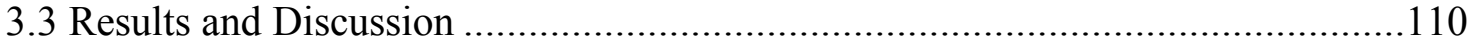

3.3.1 Design of chimaeric S100A11/S100A10 proteins.......................................110

3.3.2 Engineering of chimaeric S100A1 1 proteins …….......................................116

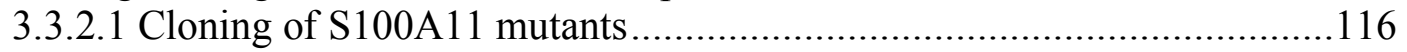

3.3.2.2 Expression and purification of S100A11 proteins...................................119

3.3.3 Characterization of chimaeric S100A11/S100A10 proteins ..........................128

3.3.3.1 Oligomerization states of chimaeric proteins..........................................128

3.3.3.2 Folded state of S100A11/S100A10 proteins........................................134

3.3.3.3 Most chimaeric $\mathrm{Ca}^{2+}$-S100A11 proteins can adopt an open

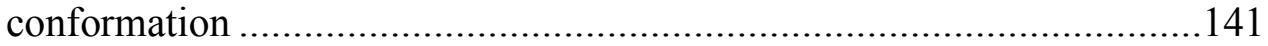

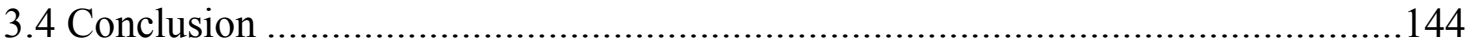

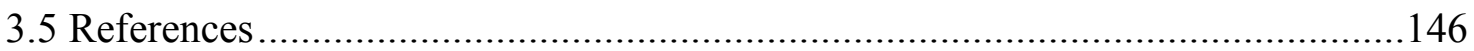

\section{Chapter 4 STRUCTURAL EFFECTS OF SUBSTITUTED \\ REGIONS IN S100A11 ……................................................................148}

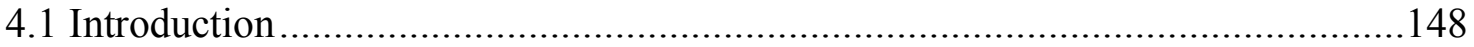

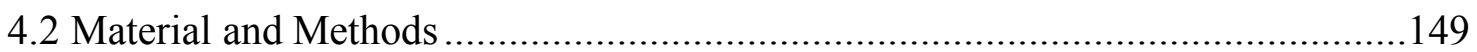

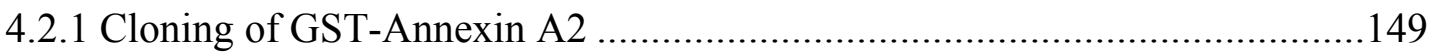

4.2.2 Protein Expression and Purification.........................................................149

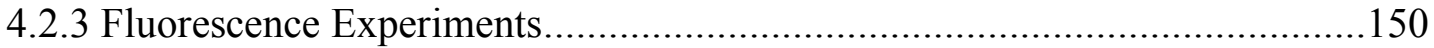

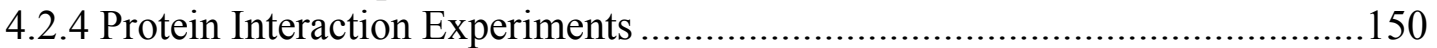

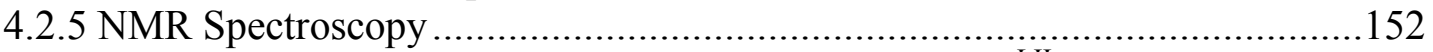

4.2.5.1 Backbone Chemical Shift Assignment of S100A11 ${ }^{\mathrm{LII}}$...........................152

4.2.5.2 Residual Dipolar Coupling.................................................................152 
4.3 Results and Discussion

4.3.1 Evidence for a $\mathrm{Ca}^{2+}$-independent hydrophobic surface in site II chimaeric proteins

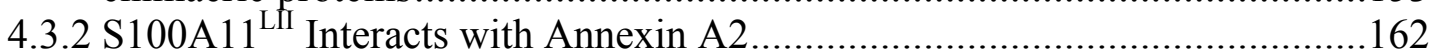

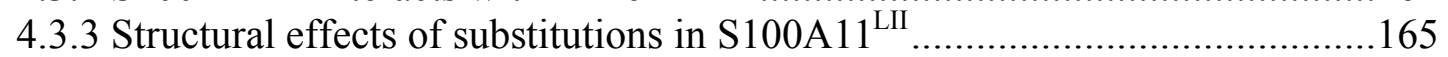

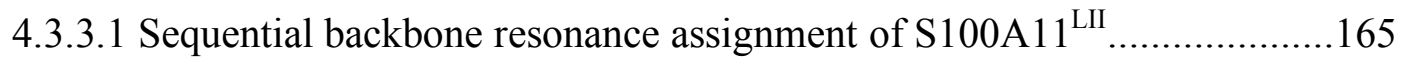

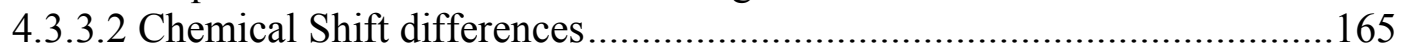

4.3.3.3 Residual Dipolar Coupling..............................................................170

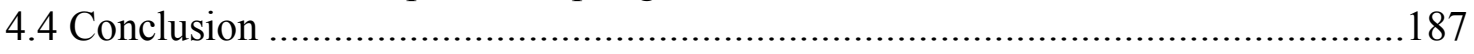

Chapter 5 ENERGETICS OF S100-PEPTIDE INTERACTIONS............................191

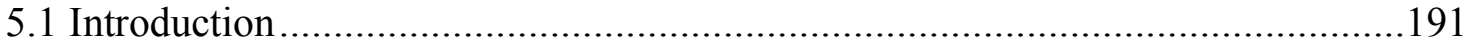

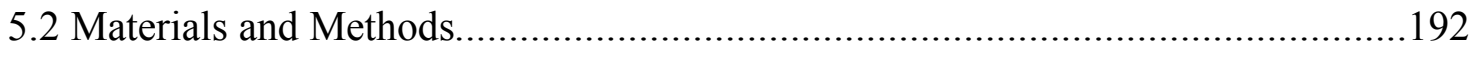

5.2.1 Protein Expression and Purification:...........................................................192

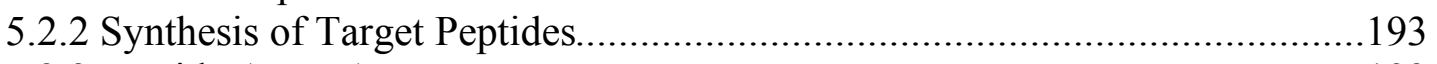

5.2.3 Peptide Array Assays …………………………......................................193

5.2.4 Isothermal Titration Calorimetry ……………….....................................195

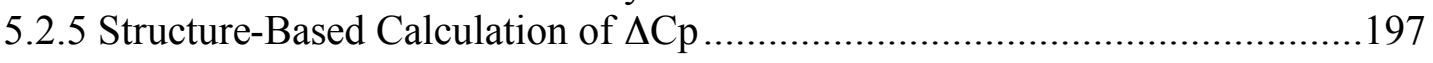

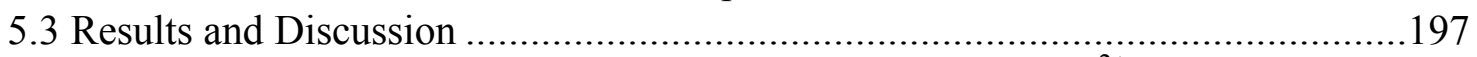

5.3.1 Binding specificity of annexins A1 and annexin A2 to $\mathrm{Ca}^{2+}$-S100A11..........197

5.3.2 Annexin $\mathrm{A} 1$ and $\mathrm{A} 2$ interactions with $\mathrm{Ca}^{2+}-\mathrm{S} 100 \mathrm{~A} 11$ are

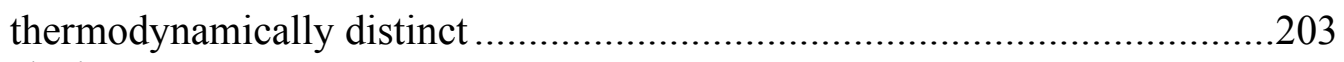

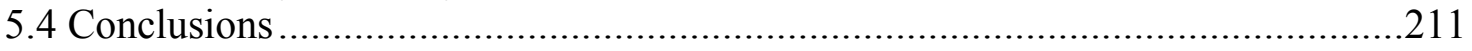

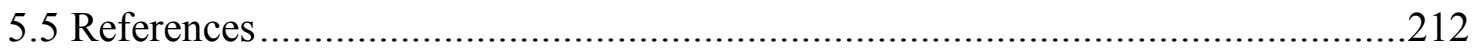

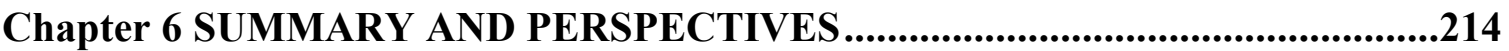

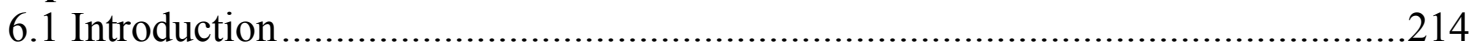

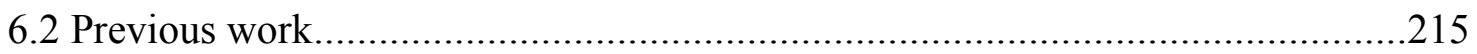

6.3 Comparative studies of S100A10 and S100A11 ….........................................216

6.4 Engineering of chimaeric S100A11/S100A10 proteins .....................................217

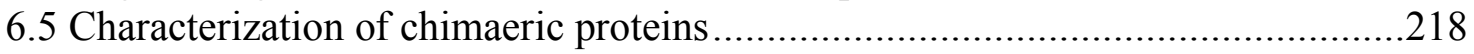

6.6 Evidence for a calcium-independent hydrophobic surface in loop II

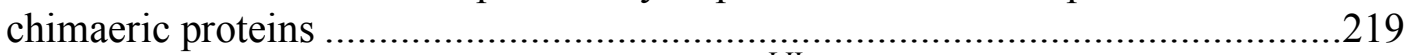

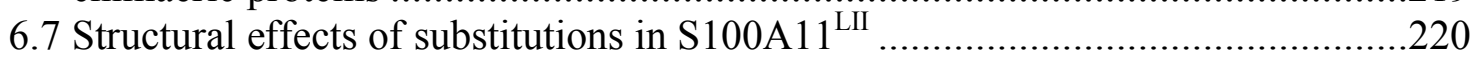

6.8 Importance of calcium-binding site II to the open conformation of S100A10 _....220

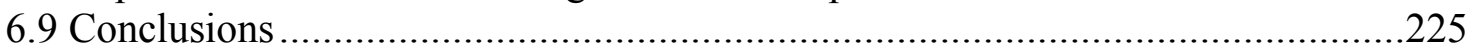

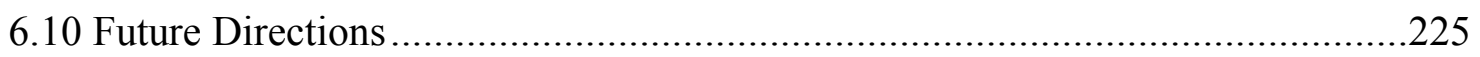

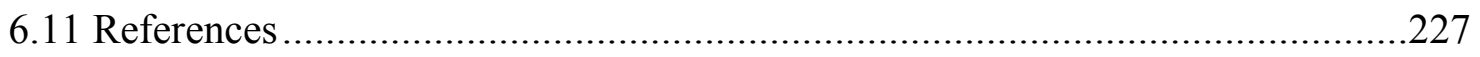

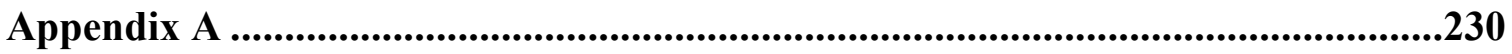

Appendix B............................................................................................................231

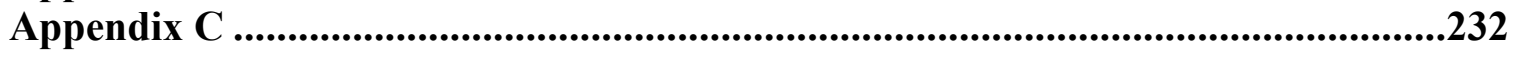

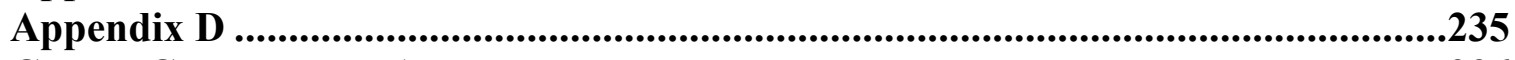

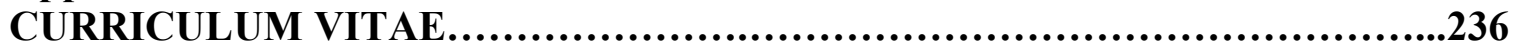




\section{LIST OF FIGURES}

Figure 1.1 Calcium homeostasis........................................................................ 2

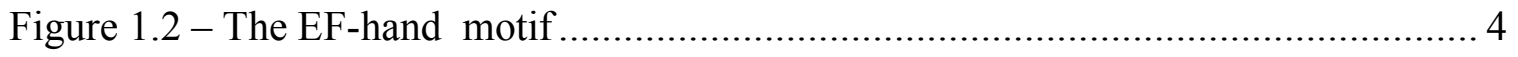

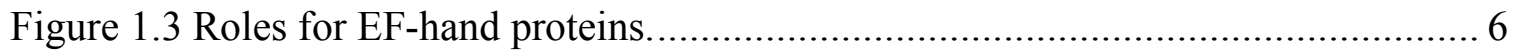

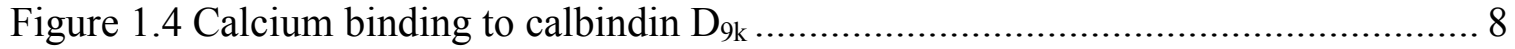

Figure 1.5 Calcium-induced conformational change in calmodulin ............................ 10

Figure 1.6 Calcium dependent and independent interactions of the S100 family........... 12

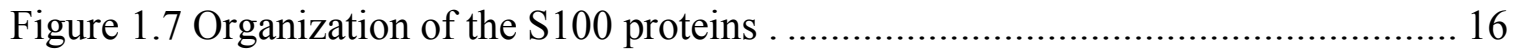

Figure 1.8 Calcium induced conformational change in the S100 proteins .................... 17

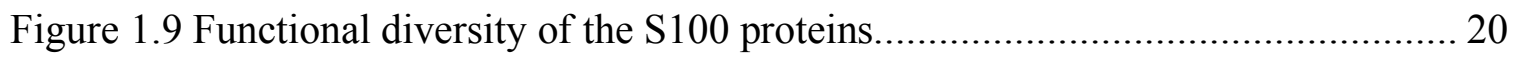

Figure 1.10 Target protein orientation for S100 proteins. ................................... 27

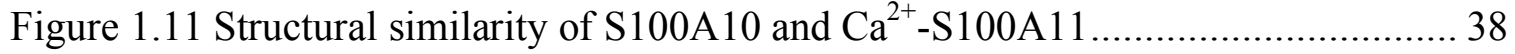

Figure 1.12 Iterative process to design S100A11/S100A10 chimaeric proteins............. 42

Figure 1.13 Sequence similarity between S100A10 and S100A11 ............................. 43

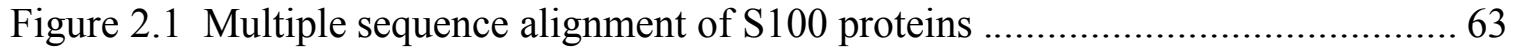

Figure 2.2 Helical wheel representations for S100A10 and S100A11_........................ 67

Figure 2.3 Structural comparison between S100A10 and S100A11........................... 69

Figure $2.4 \alpha$-Carbon distances between interhelical faces in S100A11 and S100A10_... 73

Figure 2.5 Side-chain packing in helix III interfaces from S100A10 and apo-S100A11. 76

Figure 2.6 Electrostatic surface representation of S100A11 and S100A10 proteins. ...... 78

Figure 2.7 Fractional accessible surface are for side chains in S100A10 and S100A11.. 80

Figure 2.8 Changes in fractional accessible surface area for side chains in S100A10

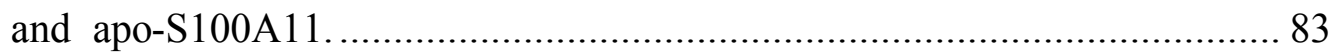

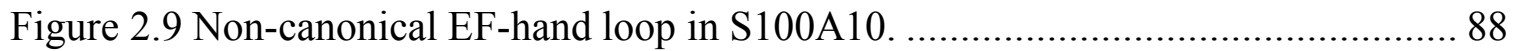

Figure 2.10 Hydrogen-bond network in loop I of S100A10 and S100A11................. 91

Figure 2.11 Inversion of the canonical loop in S100 proteins................................... 92

Figure 2.12 Stabilizing hydrogen bond network in loop II of S100A10 ..................... 94

Figure 2.13 Interactions in the linker region of S100A10 and apo-S100A11............... 96

Figure 3.1 Design of chimaeric S100A11 proteins ............................................. 111 
Figure 3.2 Incorporation of endonuclease cleavage sites in pAED4 encoding

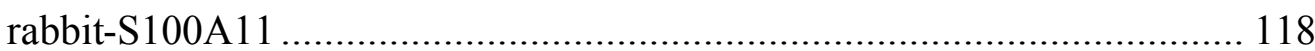

Figure 3.3 PCR product for the synthesis of chimaeric S100A11 proteins. ................. 120

Figure 3.4 Expression of recombinant S100A11 proteins. .................................... 122

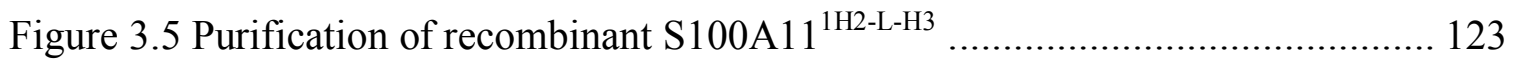

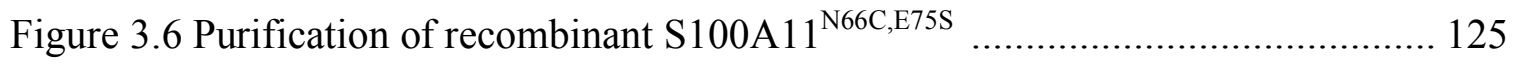

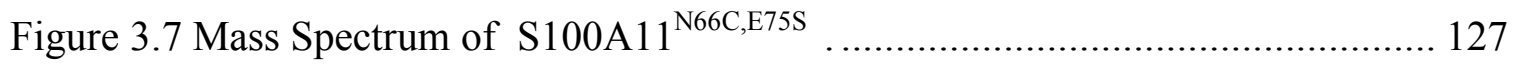

Figure 3.8 Purification of chimaeric S100A11/S100A10 proteins............................ 129

Figure 3.9 Size distribution histogram for S100A11 proteins ............................... 132

Figure 3.10 Sedimentation equilibrium curves for apo-S100A11 ......................... 135

Figure 3.11 Sedimentation equilibrium curves for chimaeric S100A11 proteins ......... 136

Figure 3.12 Secondary structure of chimaeric apo-S100A11 proteins measured by far UV-CD spectropolarimetry........................................... 138

Figure 3.13 Structural changes of chimaeric S100A11 proteins as determined using

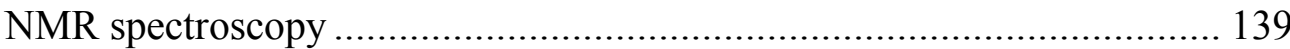

Figure 3.14 Calcium-induced conformational changes in chimaeric proteins with modified helices................................................................ 142

Figure 3.15 Calcium-induced conformational changes in chimaeric proteins with substituted calcium binding loops.

Figure 4.1 Exposed hydrophobic surface exposure of S100A10 and S100A11 proteins monitored by enhancement of ANS fluorescence.................................. 155

Figure 4.2 Observed changes in ANS fluorescence intensity of apo-S100A11 proteins 156

Figure 4.3 Exposed hydrophobic surface area of S100A10 and S100A11 proteins evaluated by their binding capacities to a phenyl Sepharose matrix. 160

Figure 4.4 Degree of apo-S100A11 proteins interaction with a phenyl Sepharose matrix 161

Figure 4.5 Interaction of S100A11 and S100A10 proteins with annexin A2 .............. 164

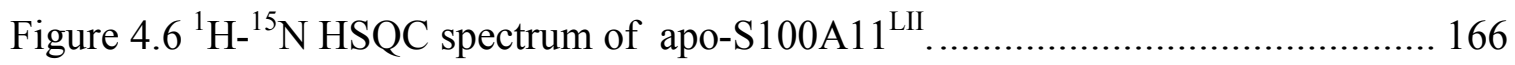

Figure 4.7 Sequential backbone assignment of S100A11 ${ }^{\text {LII }}$. 167 
Figure 4.8 Structural changes in S100A11 ${ }^{\mathrm{LII}}$ compared to apo-S100A11 ................... 169

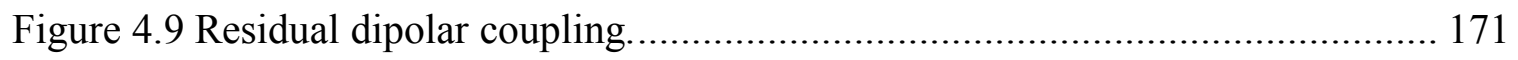

Figure 4.10 Magnetic alignment of Pf1 filamentous phage..................................... 174

Figure 4.11 Examples of the changes in ${ }^{1} \mathrm{H}^{15}{ }^{15}$ heteronuclear splittings of

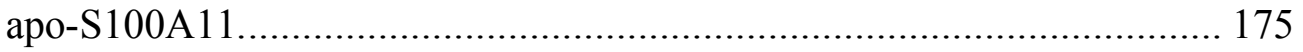

Figure 4.12 Experimental ${ }^{1} \mathrm{H}^{15} \mathrm{~N}$ dipolar coupling for apo-S100A11 ..................... 177

Figure 4.13 Apo-S100A11 structure refinement using RDCs. ................................ 178

Figure 4.14 Measurement of residual dipolar couplings for apo-S100A1 $1^{\mathrm{LII}} \ldots \ldots \ldots \ldots . . . . .180$

Figure 4.15 Experimental ${ }^{1} \mathrm{H}_{-}{ }^{15} \mathrm{~N}$ dipolar couplings for apo-S100A1 $1{ }^{\mathrm{LII}}$................ 181

Figure 4.16 Structural effects of substitution in loop II in apo-S100A11 as evidenced by RDCs.................................................................. 183

Figure 4.17 Correlation between measured and calculated ${ }^{1} \mathrm{H}-{ }^{15} \mathrm{~N}$ dipolar

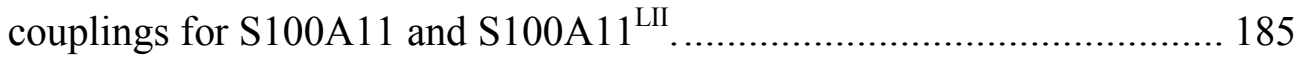

Figure 5.1 Amino acid sequences used for peptide array experiments....................... 194

Figure 5.2 Sequence alignments of the N-terminal sequences of annexin A1 and A2 ... 199

Figure $5.3 \mathrm{Ca}^{2+}$-S100A11 binding to annexin peptides shown by peptide arrays......... 201

Figure 5.4 ITC experiments for annexin peptide binding to $\mathrm{Ca}^{2+}-\mathrm{S} 100 \mathrm{~A} 11 \ldots \ldots \ldots \ldots . . . . .205$

Figure 5.5 ITC experiments for annexin A1 and A2 binding to $\mathrm{Ca}^{2+}-\mathrm{S} 100 \mathrm{~A} 11$

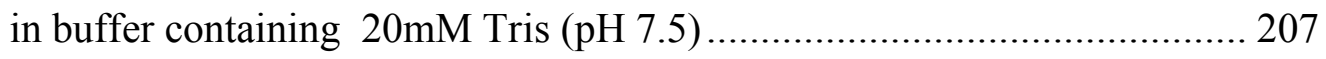

Figure 5.6 Comparison of thermodynamic parameters for S100-target interactions ..... 210 


\section{LIST OF TABLES}

Table 1.1 Calcium-dependent interactions of the S100 proteins................................ 22

Table 1.2 Calcium-independent interactions of the $\mathrm{S} 100$ proteins............................. 31

Table 1.3 Homo- and heterodimer formation of the S100 proteins.............................. 33

Table 2.1 Comparison of Interhelical angles in S100A10 and S100A11_.................... 70

Table 2.2 RMSD ${ }^{\mathrm{a}}$ on $\mathrm{C} \alpha$ positions from S100A10 and S100A11............................ 71

Table 3.1 Design of chimaeric S100A11/S100A10 proteins ................................... 115

Table 3.2 Mass determination of chimaeric proteins by electrospray

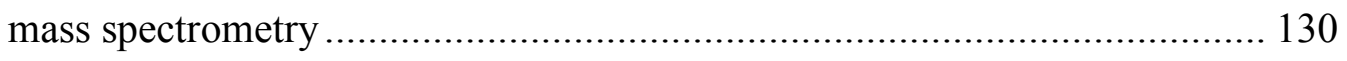

Table 3.3 Summary of oligomerization analyses for S100A11 proteins ..................... 133

Table 4.1. Correlation of experimental RDCs of S100A11 $1^{\mathrm{LII}}$ and calculated S100A11. 186

Table 5.1Thermodynamic parameters for Titrations of $\mathrm{Ca}^{2+}-\mathrm{S} 100 \mathrm{~A} 11$ with annexin A1 and $\mathrm{A} 2$ peptides 206 


\section{LIST OF APPENDICES}

Appendix A Primers used for engineering chimaeric S100A11/S100A10_..........231

Appendix B Chimaeric S100A11/S100A10 proteins designed.....................232

Appendix C Differences in surface area exposure for side chains in

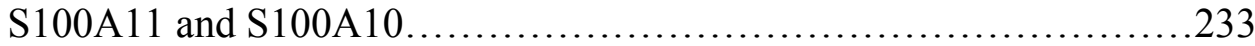

Appendix D $\alpha$-Carbon distances between interhelical faces in S100A11 and

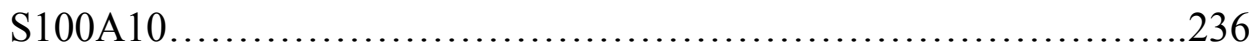




\section{LIST OF ABBREVIATIONS, SYMBOLS AND NOMENCLATURE}

\begin{tabular}{|c|c|}
\hline$\gamma$ & gyromagnetic ratio \\
\hline$\delta$ & chemical shit \\
\hline$v$ & freequency \\
\hline$\Omega$ & interhelical angle \\
\hline$\phi$ & phi angle \\
\hline$\psi$ & psi angle \\
\hline ANS & 4,4'-dianilino-1,1'-binaphthyl-5,5'disulfonic acid, dipotassium salt \\
\hline ATP & adenosine triphosphate \\
\hline Bo & magnetic field \\
\hline bp & base pair \\
\hline $\mathrm{Ca}^{2+}$ & calcium \\
\hline $\mathrm{Ca}^{2+}-\mathrm{CaM}$ & calcium-bound calmodulin \\
\hline $\mathrm{CaM}$ & calmodulin \\
\hline $\mathrm{Ca}^{2+}-\mathrm{S} 100 \mathrm{~A} 11$ & calcium-bound S100A11 \\
\hline CacyBP/SIP & calcyclin-binding protein/Siah1-interacting protein \\
\hline CapZ & actin capping protein \\
\hline $\mathrm{CD}$ & circular dichroism \\
\hline $\mathrm{D} 2 \mathrm{O}$ & deuterium oxide \\
\hline $\mathrm{Da}$ & daltons \\
\hline DLS & dynamic light scattering \\
\hline DSS & 2,2'-dimethyl-2-silapentane-5-sulfonate \\
\hline DTT & dithiothreitol \\
\hline E. coli. & Escherichia coli \\
\hline EDTA & ethylenediaminetetracetic acid \\
\hline EGTA & ethylene glycol bis(2-aminoethyl ether)-N,N,N',N' -tetraacetic acid \\
\hline ER & endoplasmic reticulum \\
\hline ESI & electrospray ionization \\
\hline GAPDH & glyceraldehydes-3-phosphate dehydrogenase \\
\hline
\end{tabular}




$\begin{array}{ll}\text { GFAP } & \text { glial fibrillary acidic protein } \\ \text { GST } & \text { glutathione S-transferase } \\ \text { HEPES } & \text { 4-(2-hydroxyethyl)-1-piperazineethanesulfonic acid } \\ \text { HPLC } & \text { high performance liquid chromatography } \\ \text { HSQC } & \text { heteronuclear single quantum coherence } \\ \text { Hz } & \text { hertz } \\ \text { IPTG } & \text { Isopropyl } \beta \text {-D-1-thiogalactopyranoside } \\ \text { IPAP } & \text { in phase, antiphase } \\ \text { ITC } & \text { isothermal titration Calorimetry } \\ \text { Kd } & \text { dissociation constant } \\ \text { kDa } & \text { kilodaltons } \\ \text { MES } & \text { 2-(N-morpholino)ethanesulfonic acid } \\ \text { MW } & \text { molecular weight } \\ \text { NDR } & \text { nuclear Dbf2-related protein kinase } \\ \text { NMR } & \text { nuclear magnetic resonance } \\ \text { OD } & \text { optical density } \\ \text { PAGE } & \text { polyacrylamide gel electrophoresis } \\ \text { PCR } & \text { polymerase chain reaction } \\ \text { RDC } & \text { residual dipolar coupling } \\ \text { rmsd } & \text { root mean square deviation } \\ \text { RyR } & \text { ryanodine receptor } \\ \text { SDS } & \text { sodium dodecyl sulfate } \\ \text { TCEP } & \text { (tris (2-carboxyethyl)phosphine) } \\ \text { Tris } & \text { tris(hydroxymethyl)aminomethane } \\ \text { Ala (A) } & \text { aspartic acid } \\ \text { Arg (R) } & \text { alanine } \\ \text { Asn (N } & \text { asparinine } \\ \text { Asp (D) } & \text { Cys (C) }\end{array}$




$\begin{array}{ll}\text { Gln }(\mathrm{Q}) & \text { glutamine } \\ \text { Glu (E) } & \text { glutamic acid } \\ \text { Gly (G) } & \text { glycine } \\ \text { His (H) } & \text { histidine } \\ \text { Ile (I) } & \text { isoleucine } \\ \text { Leu (L) } & \text { leucine } \\ \text { Lys (K) } & \text { lysine } \\ \text { Met (M) } & \text { methinonine } \\ \text { Phe (F) } & \text { phenylalanine } \\ \text { Pro (P) } & \text { propline } \\ \text { Ser (S) } & \text { serine } \\ \text { Thr (T) } & \text { threonine } \\ \text { Trp (W) } & \text { tryptophan } \\ \text { Tyr (Y) } & \text { tyrosine } \\ \text { Val (V) } & \text { valine }\end{array}$




\section{Chapter 1 \\ INTRODUCTION ${ }^{1}$}

\subsection{Calcium Signaling}

Variations of calcium $\left(\mathrm{Ca}^{2+}\right)$ levels in the cell constitute a key signal that regulates almost every aspect of cellular life, ranging from fertilization, cell growth and muscle contraction to cell death in apoptosis. The role of calcium ions in these processes is to act as "second messengers", which are molecules that transmit signals from receptors at the plasma membrane to target molecules in the cell, and thereby, leading to a desired physiological response. Due to the importance of the $\mathrm{Ca}^{2+}$ signaling system, levels of the ion need to be tightly controlled. As illustrated in Figure 1.1 in a resting cell the cytosolic calcium levels are near $10^{-7} \mathrm{M}$ while the levels in the extraceullar matrix and the intracellular organelles are close to $10^{-3} \mathrm{M}[1]$ resulting in an large $\mathrm{Ca}^{2+}$ gradient across the plasma membrane. This gradient is used during signal transduction when external stimuli allow calcium ions to flow into the cytosol leading to an increase in calcium concentration of about a hundred-fold relative to the resting state (Figure 1.1B). The influx of calcium from the extracellular matrix is controlled by voltage-gated or receptor operated channels that respond to changes in membrane potential or activation via ligand binding. Calcium can also be released from the endoplasmic reticulum (ER) where the ion is passed to the cytoplasm by the ryanodine (RyR) or inositol $(1,4,5)$-trisphosphate

\footnotetext{
${ }^{1}$ A version of this chapter has been published. Santamaria-Kisiel, L., Rintala-Dempsey, A.C. and Shaw, G.S. 2006. Calcium dependent and -independent interactions of the S100 protein family. Biochem J. 396, 201-214.
} 
A

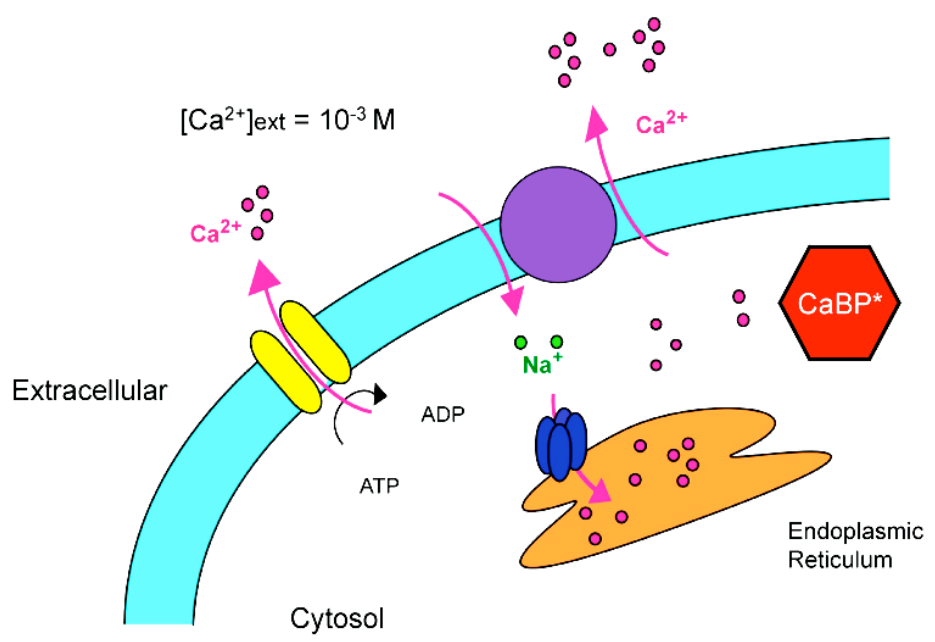

$\left[\mathrm{Ca}^{2+}\right]$ int $=10^{-7} \mathrm{M}$

B

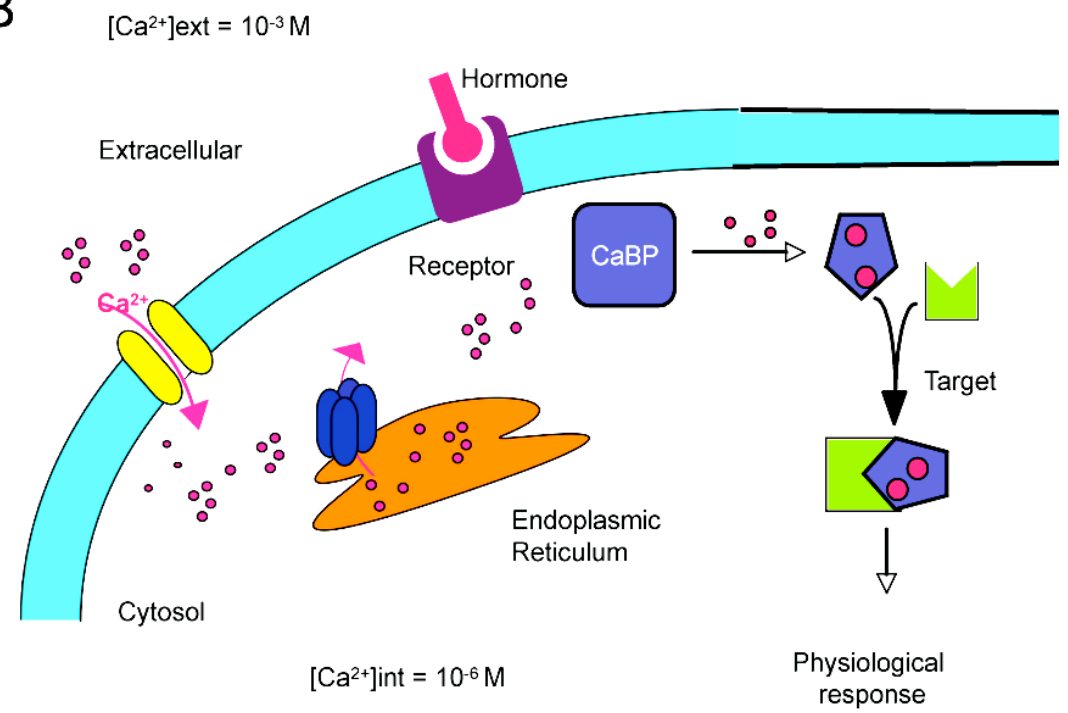

\section{Figure 1.1 Calcium homeostasis.}

(A) In the resting cell, calcium is exported to the extracellular environment via $\mathrm{Ca}^{2+}$ ATPase or $\mathrm{Na}^{+} / \mathrm{Ca}^{+}$exchange pump, or transported to intracellular compartments such as the endoplasmic reticulum where it binds to buffer calcium binding proteins $\left(\mathrm{CaBP}^{*}\right)(\mathrm{B})$ The cell is activated through hormones binding to cell surface receptors or imported through $\mathrm{Ca}^{2+}$-channels, causing a $\sim 100$ fold increase in calcium concentration. Calcium signaling proteins bind the ion allowing interaction with target molecules leading to a physiological response. 
receptors $\left(\mathrm{IP}_{3} \mathrm{R}\right)$ [2,3]. Resting calcium levels are re-established by reciprocal mechanisms such as plasma membrane pumps or exchangers or through re-entry to the endoplasmic reticulum via $\mathrm{Ca}^{2+}$-ATPases [4] once the signal is completed.

The intermediary calcium pulse that results from the transport of $\mathrm{Ca}^{2+}$ in and out of the cytoplasm stimulates a variety of cellular activities. The increased calcium level can act as a feedback inhibitor to switch the calcium import machinery off. Further, high concentrations of free intracellular $\mathrm{Ca}^{2+}$ are lowered by calcium-buffering proteins, such as calbindin $\mathrm{D}_{9 \mathrm{k}}$, parvalbumin and oncomodulin, that have high capacities for calcium, tight binding affinities (in the nanomolar range) or unique kinetic properties that act to fine tune the levels and availability of free cytosolic calcium (Figure 1.2). However, the most important events arising from the calcium signal are the triggering of biological events modulated through binding of calcium to a large number of calcium sensor proteins (Figure 1.2), including calmodulin, troponin-C and the $\mathrm{S} 100$ proteins. By far, the largest group of sensors is the EF-hand calcium-binding proteins of which more than 1000 have been identified from the human genome [5].

\subsection{EF-hand motif}

Kretsinger first identified the EF-hand motif, two $\alpha$-helices with an intervening 12-residue calcium-binding loop, more than 30 years ago [6]. The EF-hand nomenclature is adapted from the two respective helices of parvalbumin ( $\mathrm{E}$ and $\mathrm{F}$ ), which flank the calcium-binding loop (Figure 1.2A) and the fact that this motif resembles a right hand where the extended forefinger and thumb represents the helices and the closed middle finger the calcium-coordinating sphere (Figure 1.2B). 

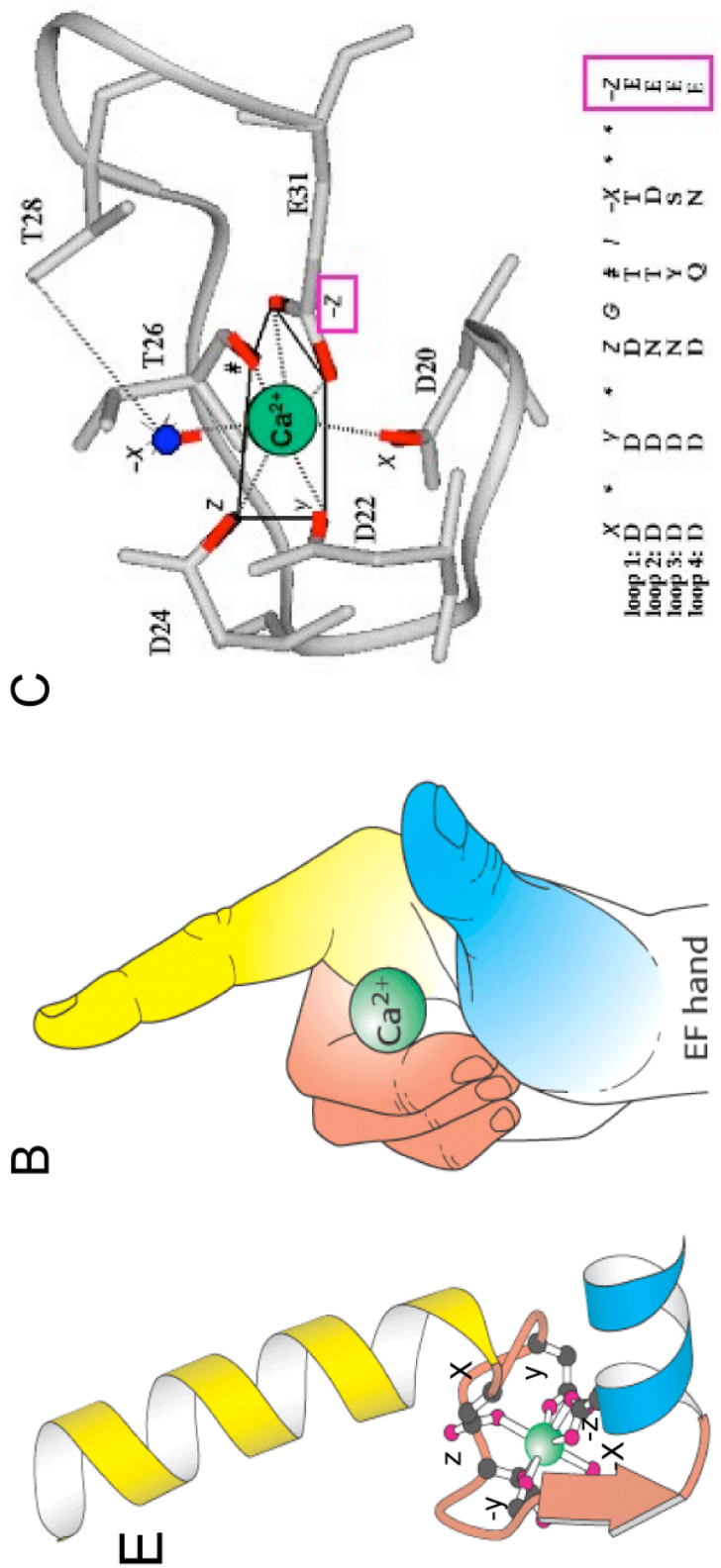

$\varangle$

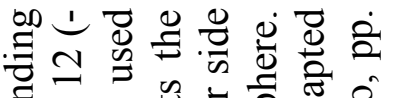

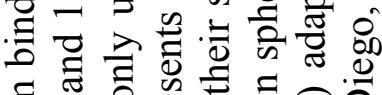

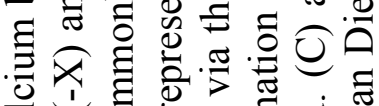

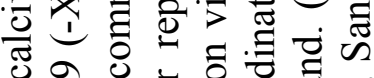
¿

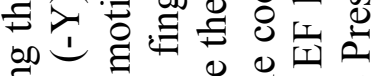
.尹口

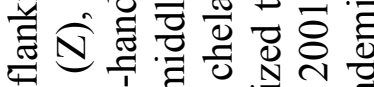
红岌

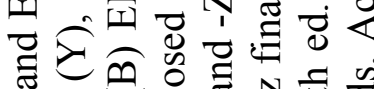

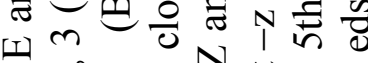

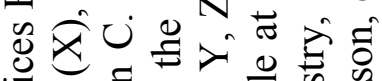

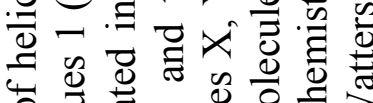
ᄂ

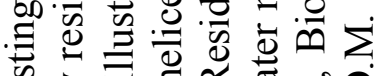

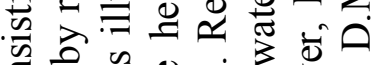

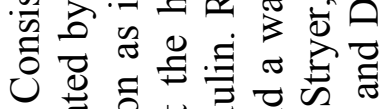
渮.

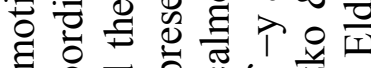

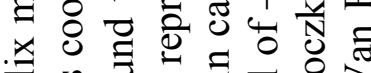
侊.

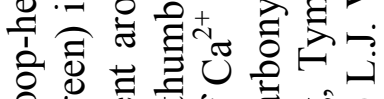

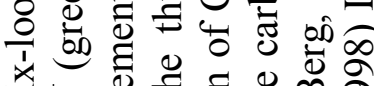

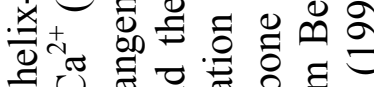
चु 氜 규 空

Ш

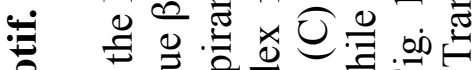

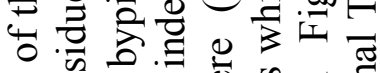
ธี

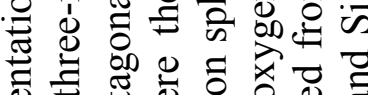
司至

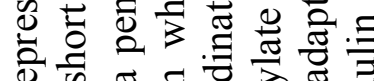

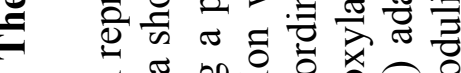

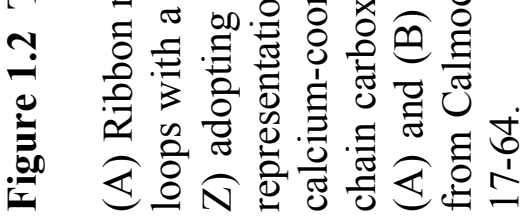


Structural analysis indicates the chelating residues in the canonical (12 residues) calcium-binding loop form a conserved pentagonal bipyramidal arrangement of seven oxygen ligands around the ion. Amino acids forming the ligands to the cation utilize the side chains at positions $1(\mathrm{X}), 3(\mathrm{Y}), 5(\mathrm{Z})$, and $12(-\mathrm{Z})$, the backbone carbonyl of position $7(-\mathrm{Y})$, and a bridged water molecule at position $9(-\mathrm{X})$ [7] (Figure 1.2C). Strong preferences exist for aspartate and glutamate in the 1 and 12 coordinating positions respectively, and glycine at the non-coordinating position 6 [8]. A $\beta$-sheet strand formed by residues 7, 8 and 9 is also a common structural element of the EF-hand motif [7].

Functional EF-hands are found in pairs [9], required for the correct folding of the proteins and unique variations of calcium binding co-operativity. Despite high similarity in their sequences, the EF-hand proteins are involved in a variety of functions. In general, two distinct roles for the EF-hand proteins can be described: as sensor or buffers (Figure 1.3). Calcium-sensor proteins, such as calmodulin, troponin- $\mathrm{C}$ and the $\mathrm{S} 100$ family [1012], undergo structural changes exposing hydrophobic target-recognition regions upon calcium binding. These proteins have calcium affinities within the micromolar range in order to keep the proteins in their calcium-free (apo-) form in a resting cell [13]. Conversely, buffer proteins such us calbindin $\mathrm{D}_{9 \mathrm{k}}$, have high affinity for calcium (in the nanomolar range), do not experience significant calcium-induced rearrangements and are responsible for maintaining the low intracellular levels of calcium [14] (Figure 1.3).

\subsection{Calbindin $D_{9 K}$}

Calbindin $\mathrm{D}_{9 \mathrm{~K}}(\mathrm{~S} 100 \mathrm{G})$ is a small $(75$ residues, $75 \mathrm{KDa})$ monomeric intestinal calcium-binding protein suggested to play roles in the uptake and transport of $\mathrm{Ca}^{2+}$. 


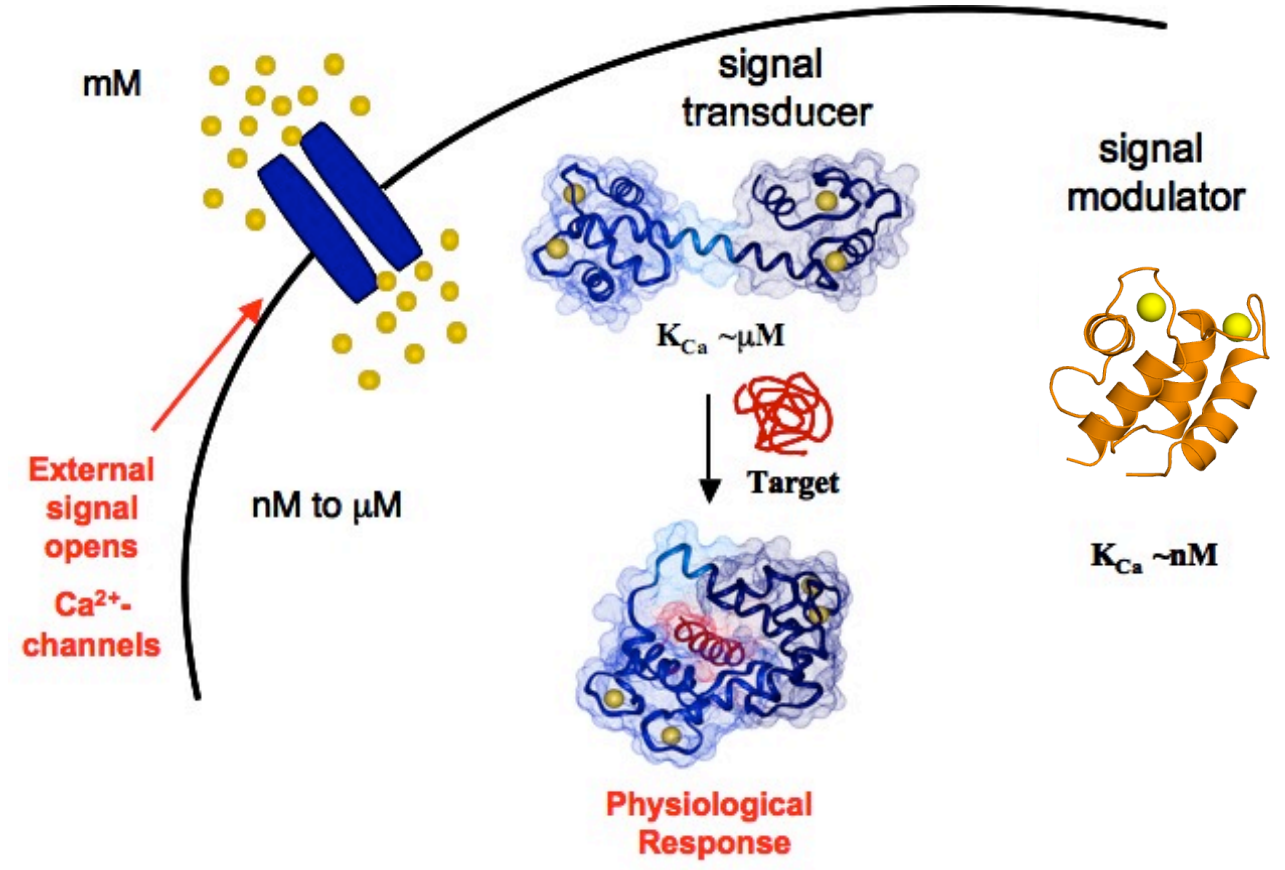

\section{Figure 1.3 Roles for EF-hand proteins.}

$\mathrm{Ca}^{2+}$-signal causes opening of the calcium channels on the cell surface causing an increase in the concentration of the ion in the cell. Calcium binding proteins such as calmodulin (structure shown in blue) bind to the cation undergoing a large conformational change leading to exposure of a hydrophobic patch that forms the target recognition surface. Calcium signaling molecules such as Calbindin $D_{9 k}$ (structure shown in orange) have affinities for calcium in the micromolar range and are activated briefly while the signal is completed. In contrast calcium-binding proteins have affinities in the nanomolar range and have a role in buffering and transporting the calcium ions. (Adapted from Akke. M and Chazin, W.J. 2001 [16]. 
It contains two high-affinity $\mathrm{Ca}^{2+}$-binding motifs $\left(10^{-9} \mathrm{M}\right)$ separated by a flexible linker [15]. The C-terminal loop is a typical, 12 residue canonical EF-hand, whereas the Nterminal loop, termed a non-canonical EF hand, contains two additional amino acids and coordinates the ion through backbone oxygens atoms [17] with calcium affinity similar to that of the C-terminal canonical site [18].

Three-dimensional structures of Calbindin $\mathrm{D}_{9 \mathrm{~K}}$ in the presence and absence of calcium $[19,20]$ show an excellent resemblance between the two forms (Figure 1.4). Binding of calcium to Calbindin $\mathrm{D}_{9 \mathrm{~K}}$ causes only minor structural rearrangements [19] involving adjustment of the backbone conformation in helices III and IV, and subtle reorganization of helix II/IV and helix III/IV interfaces but not opening of the domain nor exposure of hydrophobic surfaces as in the calcium signaling proteins calmodulin, troponin $\mathrm{C}$ and the S100 proteins (Figure 1.4) [19, 20].

\subsection{Calmodulin}

Calmodulin $(\mathrm{CaM})$ is a small (148 amino acids, $16.7 \mathrm{kDa})$, acidic protein ubiquitously expressed in all eukaryotic organism. It regulates a wide range of cellular processes including cell motility, glycogen metabolism, calcium transport, protein phosphoritalion and dephosphorilation and cell progression.

Three-dimensional structural studies of CaM [21-24] revealed a dumbbell shaped structure (Figure 1.5) formed by two lobe ( $\mathrm{N}$ - and $\mathrm{C}$ - terminal) of similar size and structure, separated by a central helix. Each lobe consists of two EF-hand motifs, binding a total of four $\mathrm{Ca}^{2+}$ ions with high affinity and cooperativity $(\mathrm{Kd} \sim 0.1-1 \mu \mathrm{M})$ [25]. Further, NMR [26, 27] and small angle X-ray scattering analyses [28] have revealed that 

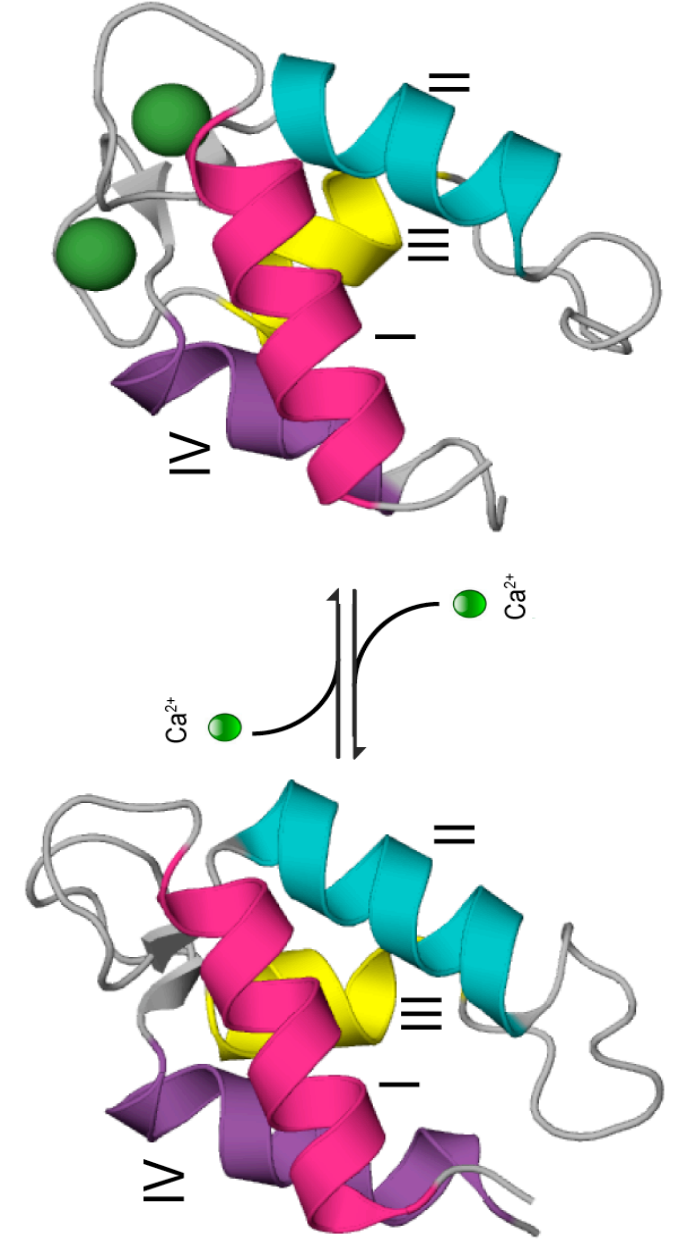

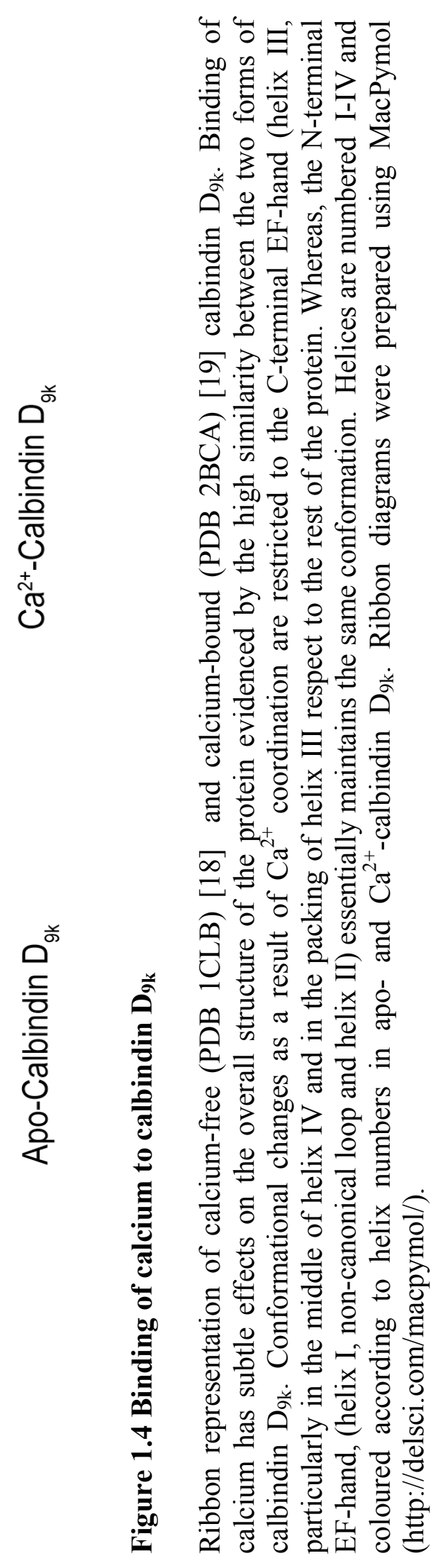

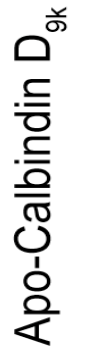


the middle part of the linking helix (residues 77-81) is unstructured and highly flexible in solution allowing the two domains to tumble independently in solution and move towards each other. The structural basis for calmodulin activation showed a calcium dependent rearrangement of its helices, resulting in the exposure of a hydrophobic surface used to recruit target proteins [21, 27, 29]. Each of the helix pairs in the globular domains (I/II; III/IV; V/VI; VII/VIII) move from a nearly antiparallel orientation (or closed conformation) in calcium-free $\mathrm{CaM}$ to a more perpendicular arrangement (open), while the orientation of adjacent helices remain similar [24] (Figure 1.5). This "opening" of the domains result in the unmasking of a previously buried hydrophobic site that is used for target and peptides recognition.

The calcium signaling role for EF-hand proteins was first established for calmodulin through its activation of 3',5'-cyclic nucleotide phosphodiesterase [30] and its ability to bind calcium [31]. Subsequently, CaM has been found to regulate numerous proteins including myosin light chain kinase [32], calcineurin [33] and calmodulin kinases [34]. Binding of the target takes place in a distinctive "wrap-around" mode of binding, where the dumbbell shape of $\mathrm{Ca}^{2+}-\mathrm{CaM}$ engulfs the target molecule, forming a globular domain facilitated by the flexible central helix $[11,16]$.

\subsection{Troponin C}

A calcium-sensitive mechanism similar to that of CaM exists for the muscle contractile EF-hand protein troponin $\mathrm{C}[35,36]$ where calcium binding modulates its interactions with troponin-I within the muscle complex. Binding of calcium to troponin $\mathrm{C}$ leads to a conformational change exposing hydrophobic residues involved in the 

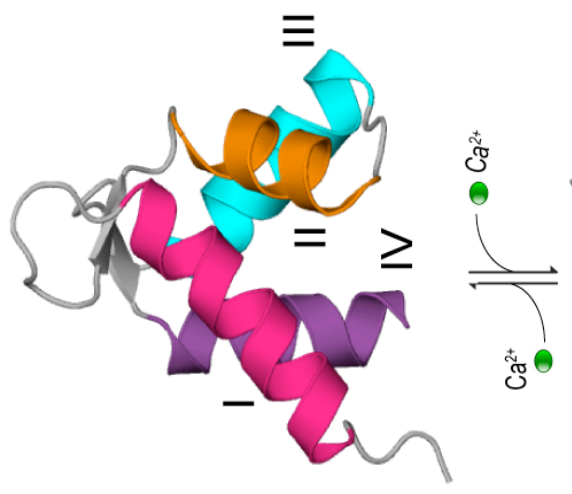

$\infty$

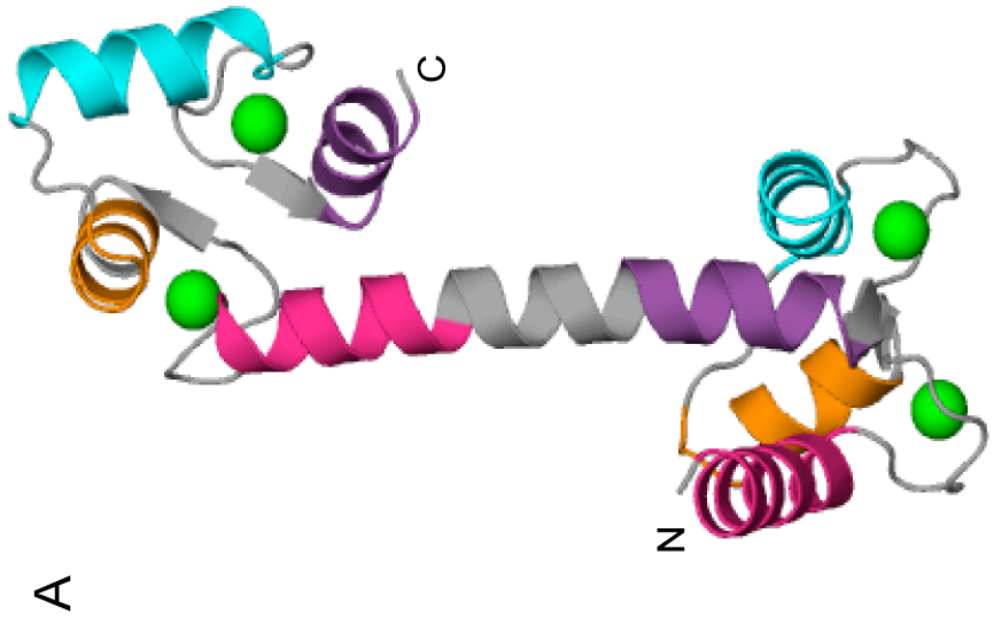

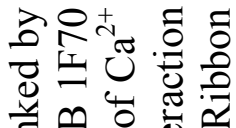

音造

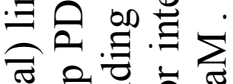

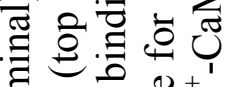

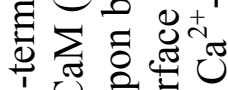

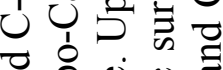
웧

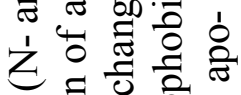

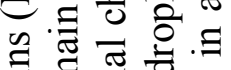

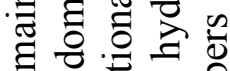
흥 뜸

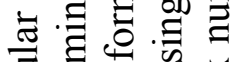

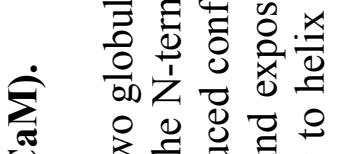
已

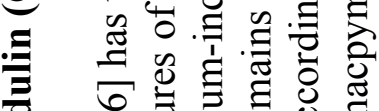

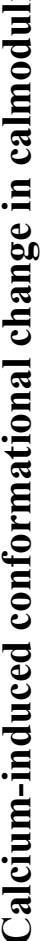
을 엉 웡 Э㤩 U듸

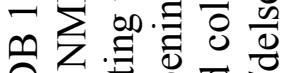

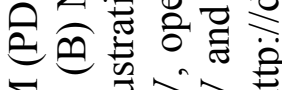

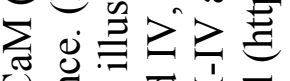
U $\sigma \overrightarrow{0}$

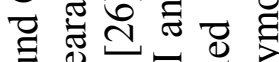

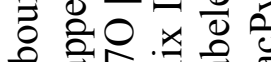

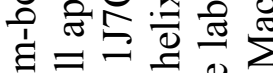

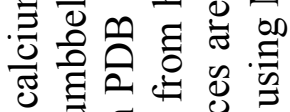
넝 5 형

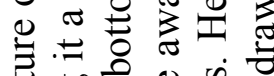
कo 0

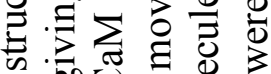
焉.

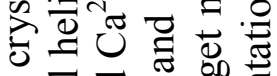

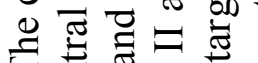

氙 还远要 
interaction with the inhibitory protein troponin I. This calcium-dependent interaction of troponin $\mathrm{C}$ with troponin I removes the troponin I inhibition of myosin ATPase, thereby modulating muscle contraction [37].

The structure of troponin $\mathrm{C}$ shows a significant resemblance with the dumbbell shaped structure of calmodulin $[38,39]$. It comprises two domains, each containing two EF-hand motifs (N-terminal helices I, II, III and IV and C-terminal helices VI, VII, VIII and IX) separated but a flexible linker. In addition troponin $\mathrm{C}$ has a 14-residue helix at its N-terminus, believed to aid with stabilization of the loop. The C-terminal domains are structural high affinity sites $\left(10^{-7} \mathrm{M}\right)$ referred as $\mathrm{Ca}^{2+}$ and $\mathrm{Mg}^{2+}$ sites, whereas the $\mathrm{N}-$ terminal loops are regulatory $\mathrm{Ca}^{2+}$ specific binding sites of low affinity $\left(10^{-5} \mathrm{M}\right)[40]$. Both structural sites are occupied during muscle contraction and relaxation, while regulatory sites bind calcium as result of increases in the concentration of the ion during contraction. Upon calcium binding helices II and III move away from I and IV [35], opening the structure and exposing a hydrophobic target recognition patch where TnI binds, thereby regulating muscle contraction.

\subsection{S100 Proteins}

In the 1990s it was established that the S100 proteins comprise a complex grouping of EF-hand calcium-sensors. These proteins have diverse tissue distributions and many protein interactions that result in multiple physiological responses [41] (Figure 1.6). Interest in the $\mathrm{S} 100$ proteins has been sparked by their involvement in several human diseases such as Alzheimer's, cancer and rheumatoid arthritis, usually due to modified levels of expression of the S100 members $[42,43]$. The S100 proteins are 


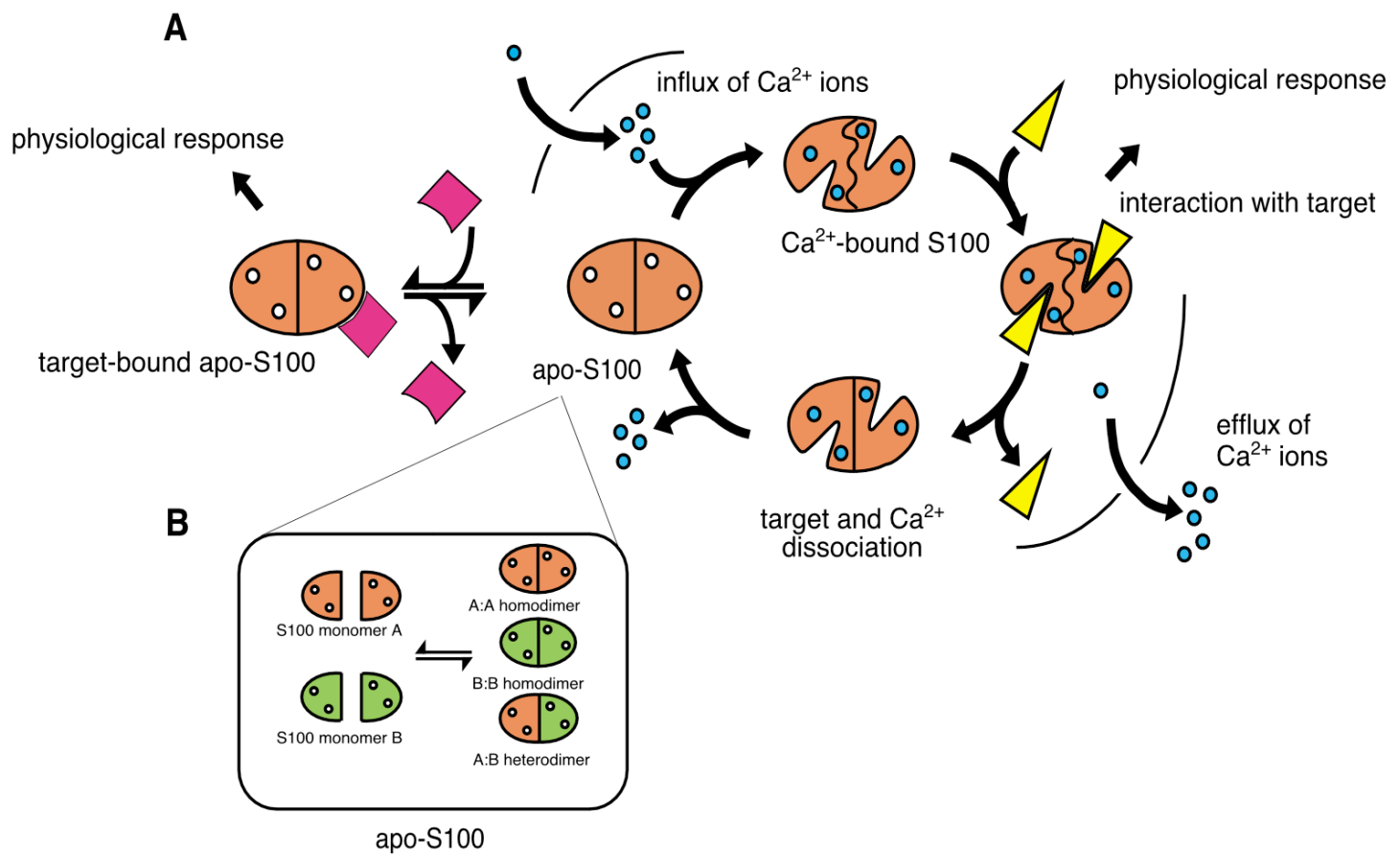

Figure 1.6 Calcium dependent and independent interactions of the S100 family.

S100 proteins generate diverse physiological responses by interacting with target molecules (pink and yellow). The majority of S100-target interactions are calciumdependent (yellow target). Only a few members have shown $\mathrm{Ca}^{2+}$-independent interactions (with pink target). Influx of calcium ions in the cytoplasm causes a conformational change in the S100 proteins, which allows interaction with $\mathrm{Ca}^{2+}$ dependent target molecules. Binding surfaces on both subunits allow the binding of two target molecules per dimer. (B) Dimerization of the S100 protein is essential for target binding and hence their physiological functions. In-vitro and in-vivo studies have shown the ability of the S100s to exist as homo- and heterodimers. 
small acidic proteins (10-12 kDa) found exclusively in vertebrates [44].

With at least 25 members found to date in humans, the S100 proteins constitute the largest subfamily of the EF-hand proteins. Of these, 21 family members (S100A1S100A18, trichohylin, filaggrin, repetin) have genes clustered on chromosome 1q21, while other S100 proteins are found on chromosomes 4p16 (S100P), 5q14 (S100Z), $21 \mathrm{q} 22$ (S100B) and Xp22 (S100G). First identified by Moore in 1965 [45], the S100 proteins have $25-65 \%$ identity at the amino acid level and contain two EF-hand motifs flanked by conserved hydrophobic residues and separated by a linker region [44]. The sequences of the linker region and the $\mathrm{C}$-terminal extension are the most variable amongst the S100 proteins.

Three features are unique to the S100 proteins when compared to other EF-hand proteins. First, the two EF-hands in each protomer differ in sequence and mechanisms of calcium coordination. The 12-residue C-terminal EF-hand ligates calcium in a similar manner to calmodulin and troponin-C resulting in a higher calcium affinity site with $\mathrm{K}_{\mathrm{d}} \sim$ $10-50 \mu \mathrm{M}[46]$. The N-terminal or non-canonical (also S100 specific) EF-hand is formed by 14 residues and binds calcium mostly through main chain carbonyls, except for the bidentate side chain of glutamate at the last position of the loop. This results in a weaker calcium affinity with $K_{d} \sim 200-500 \mu \mathrm{M}$ [46]. This presents an intriguing scenario for the S100 proteins in the cell whereby the C-terminal EF-hand has an affinity that would allow it to be populated during calcium influx while the N-terminal site affinity is likely too weak to bind calcium at any appreciable level in the absence of a target protein. However, it has been shown that several S100 proteins can increase their affinities for 
calcium up to $\sim 400$ fold $[47,48]$ upon binding of targets, and could probably clarify the functionality of the low calcium-affinity site in the S100 proteins. The second unique characteristic of the $\mathrm{S} 100$ proteins is their dimeric nature. In vivo and in vitro experiments have shown that the S100 proteins can form non-covalent homo- and heterodimers (Figure 1.6) and higher order oligomers. This indicates that dynamic exchange of the S100 subunits may occur, depending on the populations of the individual S100 protein members in a cellular compartment (Figure 1.6). Finally, S100 proteins are expressed in a tissue and cell specific fashion [41]. For example S100A1 and S100A2 are found in the cytoplasm and nucleus respectively of smooth muscle cells [49], while S100P is located in the cytoplasm of placental tissue $[50,51]$. Together this results in a complex picture of calcium signaling by the S100 proteins governed by the interchange of homo- and heterodimer protein species, calcium binding to the proteins, interaction with target proteins, cell specificity and regulation of biological function (Figure 1.6).

\subsubsection{Structures of $S 100$ proteins}

In the past 15 years three-dimensional structures of S100 proteins have been determined in the calcium-free (apo) [52-63], calcium-bound [12, 56, 64-73] and targetbound states [57, 73-78]. These structures have revealed that the S100 proteins undergo a significant calcium-induced conformational change that results in the exposure of a hydrophobic surface allowing interaction with a target protein, much like calmodulin and troponin C. In all S100 structures determined to date, with the exception of S100G (calbindin $\mathrm{D}_{9 \mathrm{~K}}$ ) the $\mathrm{S} 100$ protein exists as a symmetric dimer, each protomer containing two EF-hand motifs (Figure 1.7). The N-terminal EF-hand comprises helix I, pseudo 
calcium-binding site I and helix II, separated by a flexible linker from the C-terminal EF-hand that includes helix III, calcium-binding site II, and helix IV. The dimer interface consists of helices I (I') and IV (IV') of each subunit arranged in a bicornate or X-type four-helix bundle (Figure 1.7). The interhelical relationship between these helices is strictly maintained in both the apo- and calcium-bound states. Calcium binding to site I results in only minor alterations of its backbone conformation, consistent with the site adopting a "calcium ready" state identified many years earlier for the EF-hand protein S100G (calbindin D9k [19]. The apo-S100 proteins have a more "closed" arrangement of helices III and IV. Calcium binding causes helix III to reorient and repack itself forming a more "open" structure (Figure 1.8). For example, in apo-S100A11 helix III is nearly antiparallel to helix IV $\left(154^{\circ}\right)$ but opens by approximately $40^{\circ}$ upon calcium binding with respect to both helices II and IV $[52,73]$. The degree of opening is similar to that observed for the $\mathrm{C} / \mathrm{D}$ helices in the N-terminal domains of both troponin- $\mathrm{C}\left(60-70^{\circ}\right)$ [35] and calmodulin $\left(35-40^{\circ}\right)$ [29], although these latter proteins have additional helix movements (i.e. helix B in troponin-C) that do not occur in the S100 proteins. Nevertheless, the calcium-induced structural change in the S100 proteins results in an exposure of residues from helices III and IV in the C-terminal EF-hand, and linker region that facilitates target protein interaction.

\subsubsection{Biological roles of S100 proteins}

S100 proteins are proposed to have intracellular and extracellular roles in the regulation of many diverse processes such as protein phosphorylation, cell growth and 
A Helix I Sitel Helix II Linker HelixIII Sitell Helix IV
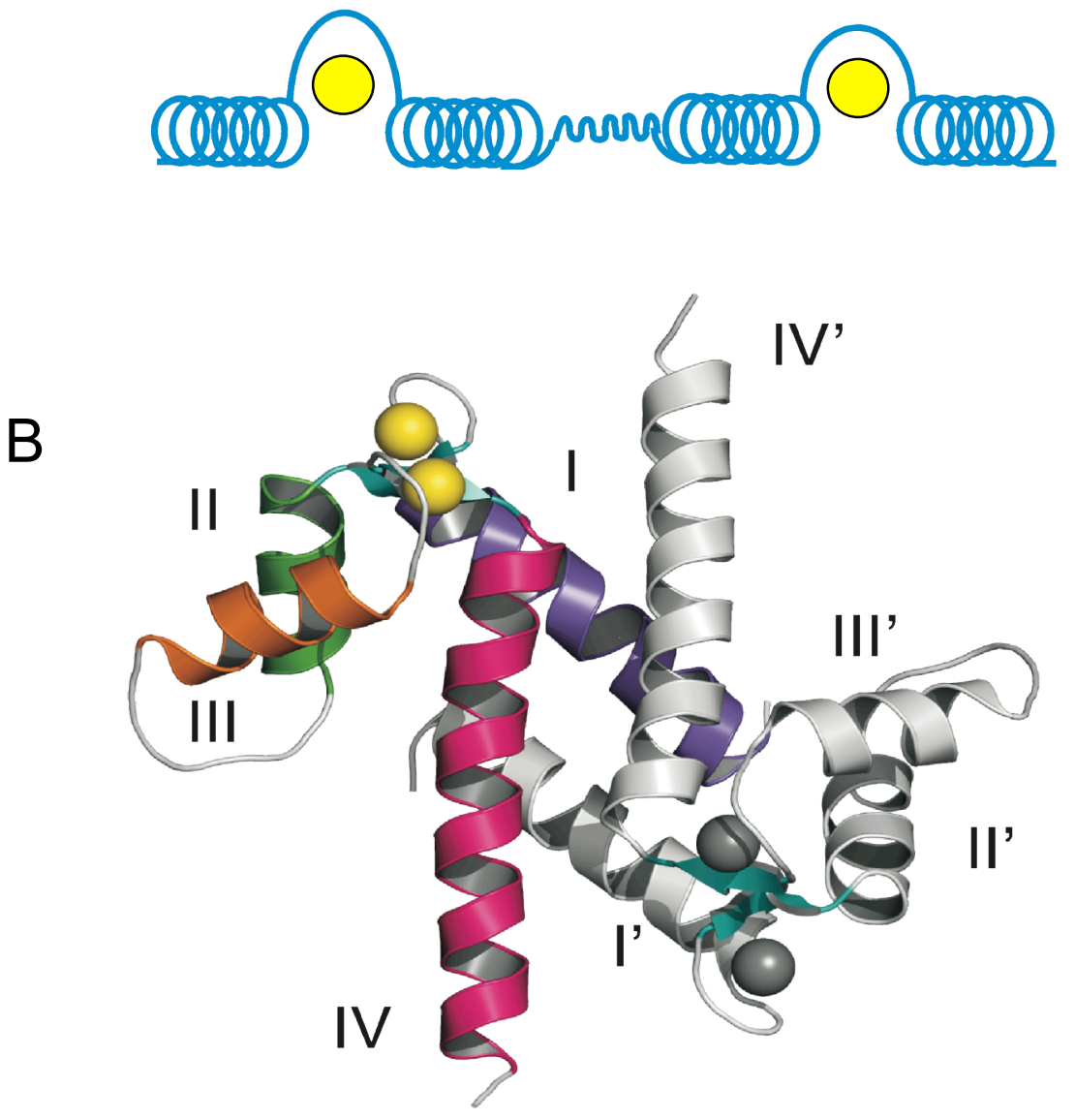

Figure 1.7 Organization of the S100 proteins .

(A) Schematic representation of a subunit organization in an S100 protein. Two EF-hand domains connected by a flexible linker. The N-terminal EF-hand is composed of helix I, non-canonical (14 residues) calcium-binding site I and helix II. The C-terminal EF-hand is composed of helix III, a canonical EF-hand loop site and helix IV. (B) Ribbon diagram of calcium-bound S100A11 ( $\mathrm{Ca}^{2+}$-S100A11) (PDB 1QLS) [73]. Helices are numbered I IV and I'-IV'. One protomer is shaded grey and the other is colored according to helices numbers. Note the X-type bundle formed by helices I, I', IV and IV' from symmetrical protomers. Ribbon representation was prepared using MacPymol (http://delsci.com/macpymol/). 

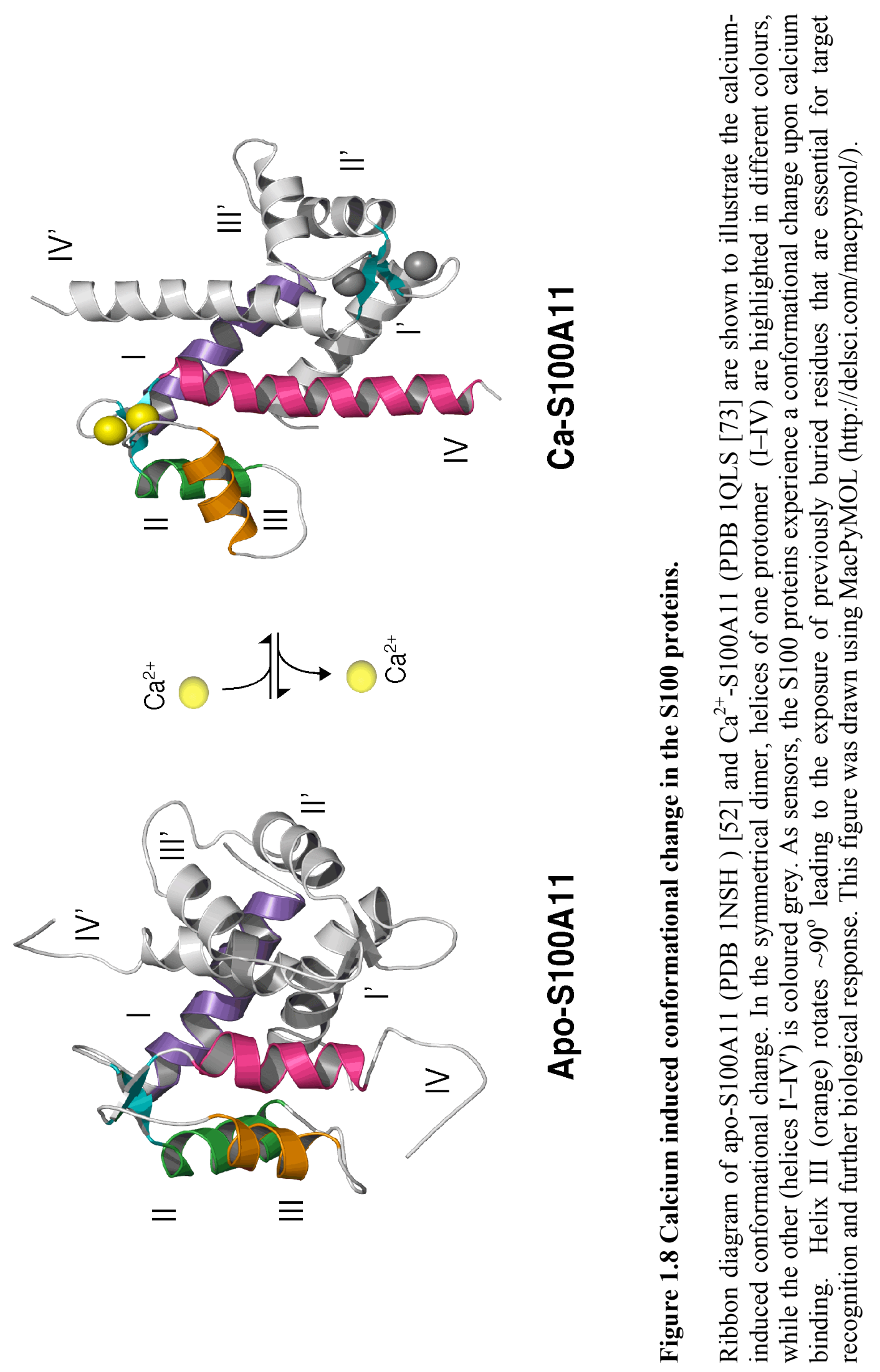
motility, cell cycle regulation, transcription, differentiation and cell survival [41]. In the past few years a wealth of information has become available supporting these diverse biological roles, and concentrating on quantifying direct interactions between an S100 protein and its biological target. Further, in vivo methods such as the yeast two-hybrid and co-immunoprecipitation have uncovered a wide spectrum of new biological targets and roles for the S100 proteins raising many interesting questions about the calciumdependent and independent functions of some S100 family members, Despite the extensive summary of S100 protein interactions, biological targets have not yet been identified for S100A3, S100A5, S100A14-S100A18, S100G (calbindin D9k), S100Z and the epidermal proteins filaggrin, trichohyalin and repetin.

In general the biological roles of the $\mathrm{S} 100$ proteins can be divided into 5 major functional groupings; (i) regulation of phosphorylation mediated by protein kinases, (ii) modulation of enzymatic activity, (iii) maintenance of cell shape and motility, (iv) influence of some signal transduction pathways and (v) promotion of calcium homeostasis. For example, S100B has been implicated in the phosphorylation of tau protein [79], and the modulation of kinase activity by NDR and protein kinase II [74, 7981]. The mechanism of this inhibition is through direct interaction with the kinase rather than recruitment of substrate. The regulation of the yeast ubiquitination pathway protein Sgt1 by S100A6 appears to operate by a similar mechanism [82]. The enzymatic activity of aldolase (isoforms A and C) and phosphoglucomutase, occurs via interactions with S100A1 [83, 84] or S100B [83, 84] while MetAP2 interacts with S100A4 [85]. It is surprising that different S100 proteins can promote opposite biological function. For 
example, the interaction of S100A1 with phosphoglucomutase inhibits enzyme activity whereas S100B interaction seems to stimulate this enzyme's function [84]. Further, $\mathrm{S} 100 \mathrm{~A} 1$ binding to aldolase $\mathrm{C}$ requires calcium, while the interaction with aldolase $\mathrm{A}$ is calcium insensitive [83]. Figure 1.9 summarizes some of the biological functions proposed for the S100 proteins.

\subsubsection{Calcium dependent protein interactions}

The calcium dependent signaling roles of the S100 proteins arise because their affinities for calcium are comparable to the free calcium concentration in the cytoplasm during a calcium wave $(\sim 1 \mu \mathrm{M})$. Thus, the binding of S100 proteins to their targets in the presence of calcium and their release, or lack of binding with EDTA, have been used to provide evidence of a calcium-dependent interaction using in vitro techniques such as affinity chromatography, optical biosensor and gel overlay, amongst others. Despite more than 90 potential protein complexes for the S100 proteins (Table 1.1), only two examples are available that use a yeast two-hybrid screen to identify calcium dependent interactions of S100A4 with the cell growth regulator protein CCN3 [86], and methionine aminopeptidase, MetAP2 [85]). The lack of calcium-dependent interactions observed in yeast is likely a function of their tightly controlled intracellular calcium levels $(\sim 200$ $\mathrm{nM}$ ) [87] that are well below the calcium dissociation constants of most S100 proteins $(\sim 10-50 \mu \mathrm{M})[46]$ and resulting in very low populations of the $\mathrm{Ca}^{2+}$-bound $\mathrm{S} 100$ protein during these experiments. S100 proteins have been found to interact with biological targets using co-immunoprecipitation or co-localization experiments. For instance, S100A2 and its interacting partner p53 co-localize in the nucleus and the cytoplasm in 


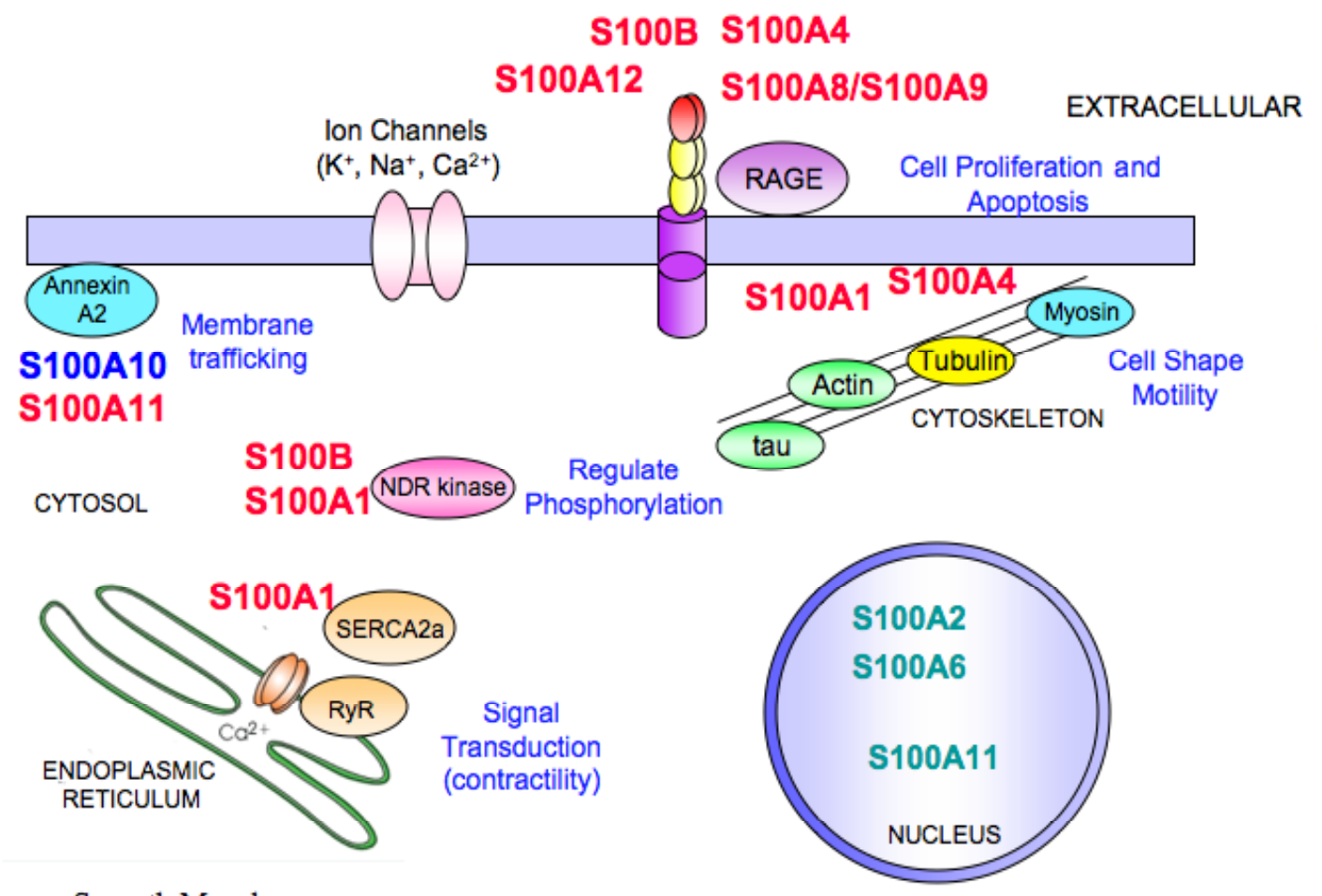

Tissue $=$ Smooth Muscle

\section{Figure 1.9 Functional diversity of the S100 proteins.}

S100 proteins act as calcium-signaling molecules (except for S100A10) transducing $\mathrm{Ca}^{2+}$ signals into biological responses. S100 proteins are distributed in cell specific manner and regulate a wide variety of intra- and extracellular processes as via interaction with target. Figure adapted from a calmodulin version of the figure courtesy of Dr. Danton H O’Day at University of Toronto. 
oral carcinoma cell lines, suggesting a relevant interaction of these two proteins [88]. Furthermore, binding of p53 to S100A2 was confirmed using pull down assays and electrophoretic shift assays [88].In contrast, S100B interactions with p53 [78], MARCKS [107] and caldesmon [108] have been observed in vitro, but no evidence of colocalization has been demonstrated [41]. This lack of co-localization evidence not only for $\mathrm{S} 100 \mathrm{~B}$, but other $\mathrm{S} 100$ proteins and their targets, has generated some confusion regarding the biological relevance of some in vitro findings.

The most significant number of protein interactions for the S100 proteins are with components of the cytoskeleton including the tubulins, intermediate filaments, actin, myosin and tropomyosin. For example, S100B controls the assembly of microtubules [109] and GFAP [110], interacts with the tubulin homolog FtsZ [111] and is proposed to regulate actin filament extension through interaction with CapZ [112]. Other S100 proteins including S100A1 S100A2, S100A4, S100A6 and S100A11 have been shown to impact similar components of the cytoskeleton (Table 1.1). In particular elegant mechanisms for calcium-dependent membrane aggregation, important for cell vesiculation have been proposed for the interaction of S100A11 with annexin A1 [113]. Several S100 proteins have been implicated in a variety of signal transduction pathways. For example, S100B, S100A1, S100A12, S100A8/S100a9 and S100P can bind to the RAGE receptor [114-116], activating an intracellular signal cascade that contributes to cell proliferation and survival. Finally, the involvement of the S100 proteins on calcium homeostasis has been suggested through interactions of S100A1 with the ryanodine receptor $[117,118]$. 
Table 1.1 Calcium-dependent interactions of the S100 proteins.

\begin{tabular}{|c|c|c|c|c|c|}
\hline PROTEIN & TARGET & SUPPORT $^{\mathrm{a}}$ & REGION $^{b}$ & $\mathrm{~K}_{\mathrm{d}}(\mathrm{nM})^{\mathrm{C}}$ & FUNCTION $^{\mathrm{d}}$ \\
\hline \multirow[t]{21}{*}{ S100A1 } & Aldolase C & GO & & & Stimulation of aldolase $\mathrm{C}$ activity \\
\hline & Annexin A5 & $\mathrm{F}, \mathrm{CC}$ & & & \\
\hline & Annexin A6 & $\mathrm{CL}, \mathrm{Cl}, \mathrm{F}, \mathrm{CC}$ & $\square \square$ صи & sub $\mu \mathrm{M}$ & Regulation of calcium flux and IF assembly \\
\hline & CacyBP/SIP1 & $\mathrm{GO}, \mathrm{AC}, \mathrm{F}, \mathrm{Cl}$ & & & Regulation of CacyBP/SIP \\
\hline & Caldesmon & $\mathrm{E}, \mathrm{F}, \mathrm{CC}$ & & 170 & Decrease in inhibition of actomyosin by caldesmon \\
\hline & CapZ (TRTK-12) & $\mathrm{GO}, \mathrm{M}, \mathrm{CC}, \mathrm{F}, \mathrm{CS}, \mathrm{O}$ & $\rightarrow \square \square \operatorname{mb} \square$ & 5550 & Modulation of actin organization \\
\hline & Desmin & $\mathrm{F}, \mathrm{O}$ & & & Inhibition of desmin intermediate filament assembly \\
\hline & F-actin & $\mathrm{CL}, \mathrm{CS}$ & & & Regulation of actin filament polymerization \\
\hline & GFAP & $\mathrm{F}, \mathrm{CC}, \mathrm{CS}$ & $\rightarrow \square \square$ пи $\square$ & 500 & Inhibition of GFAP assembly \\
\hline & Hsp70, Hsp90 [89] & $\mathrm{Cl}$ & & & Chaperone Activity \\
\hline & Microtubules/tubulin & $\mathrm{F}, \mathrm{CC}, \mathrm{O}$ & $\rightarrow \square \square$ पि口 & 6000 & Regulation of MT dynamics \\
\hline & MyoD & $\mathrm{AC}, \mathrm{CC}, \mathrm{E}, \mathrm{F}, \mathrm{Cl}$ & & 2 & Inhibition of MyoD phosphorylation and DNA binding \\
\hline & NDR & $\mathrm{O}$ & & & Activation of NDR kinase activity \\
\hline & p53 & $\mathrm{F}, \mathrm{CS}$ & $\rightarrow \square \square$ 口и & & Disruption of tubulin-S100A1 complex formation \\
\hline & Phosphoglucomutase & $\mathrm{GO}, \mathrm{AC}, \mathrm{O}$ & & & Inhibition of phosphoglucomutase activity \\
\hline & RAGE & $\mathrm{O}$ & & & Promotion of cell survival \\
\hline & Ryanodine receptor & $\mathrm{Cl}, \mathrm{OB}, \mathrm{AC}, \mathrm{O}$ & man & 214 & Regulation of RyR and cardiac contractility \\
\hline & SERCA2a and PLB & $\mathrm{AC}, \mathrm{Cl}, \mathrm{CL}, \mathrm{CE}$ & & & Regulation of EC-coupling in the heart \\
\hline & Synapsin I & $\mathrm{CL}, \mathrm{C}, \mathrm{AC}, \mathrm{OB}$ & & 245 & Regulation of catalytic activity of synapsins \\
\hline & Titin (PEVK domain) & $\mathrm{GO}, \mathrm{OB}, \mathrm{O}$ & & & Inhibition of the actin- or nebulin-PEVK interaction \\
\hline & Twitchin Kinase & $\mathrm{OB}, \mathrm{O}$ & & & Activation of twitchin kinase \\
\hline \multirow[t]{5}{*}{ S100A2 } & $\Delta \mathrm{Np63}$ & $\mathrm{O}$ & & & Downstream mediation of $\Delta \mathrm{Np} 63$ \\
\hline & Hop [90] & $\mathrm{AC}, \mathrm{Cl}, \mathrm{O}$ & & & Participation in protein folding \\
\hline & p53 & $\mathrm{CL}, \mathrm{Cl}, \mathrm{AC}, \mathrm{O}$ & & & Activation of p53 transcriptional activity \\
\hline & Tropomyosin & $\mathrm{CC}, \mathrm{Cl}, \mathrm{CL}, \mathrm{AC}, \mathrm{O}$ & & & Modulation of the actin cytoskeleton organization \\
\hline & RAGE [91] & $\mathrm{OB}, \mathrm{O}$ & & 89.5 & \\
\hline \multirow[t]{10}{*}{ S100A4 } & CCN3 & $\mathrm{Y} 2 \mathrm{H}, \mathrm{AC}$ & & & Modulation of S100A4 affinity to calcium and function \\
\hline & F-actin & CS & & 34000 & \\
\hline & MetAP2 & $\mathrm{AC}, \mathrm{CL}, \mathrm{Cl}, \mathrm{Y} 2 \mathrm{H}$ & & & Regulation of MetAP2 \\
\hline & Myosin heavy chain II -A & $\begin{array}{l}\mathrm{AC}, \mathrm{M}, \mathrm{Cl}, \mathrm{FT}, \mathrm{GO}, \mathrm{CS} \\
\mathrm{CL}, \mathrm{C}, \mathrm{OB}, \mathrm{O}\end{array}$ & $\neg \square \square$ & $220-600$ & Regulation of cytoskeletal dynamics \\
\hline & Myosin heavy chain II -B & $\mathrm{CS}, \mathrm{O}$ & & 23100 & \\
\hline & RAGE [92] & $\mathrm{OB}, \mathrm{O}$ & & $110-166$ & Induction of S100A4 neurite outgrowth function \\
\hline & Tropomyosin isoform2 & $\mathrm{AC}, \mathrm{CL}$ & & & Regulation of Tropomyosin-actin association \\
\hline & p37 & $\mathrm{F}, \mathrm{AC}$ & & & Increase affinity of S100A4 to calcium \\
\hline & p53 & $\mathrm{OB}, \mathrm{Cl}, \mathrm{CE}, \mathrm{AC}, \mathrm{F}, \mathrm{O}$ & & $8.6-14$ & Enhancement of p53-dependent apoptosis in tumors \\
\hline & Sept2, Sept6 and Sept7 & $A C$ & & & \\
\hline S100A5 & RAGE [93] & OB & & 6590 & \\
\hline \multirow[t]{18}{*}{ S100A6 } & Annexin A1 [94] & $\mathrm{F}, \mathrm{O}$ & & 17000 & \\
\hline & Annexin A11 & $\mathrm{AC}, \mathrm{GO}, \mathrm{CC}, \mathrm{Cl}, \mathrm{CL}, \mathrm{M}, \mathrm{O}$ & $\rightarrow \square$ वим & 1610 & Regulation of Annexin A11 function \\
\hline & Annexin A2 & $\mathrm{AC}, \mathrm{GO}$ & & Very weak & \\
\hline & Annexin A5 & GO & & Very weak & \\
\hline & Annexin A6 & $A C, O$ & & & \\
\hline & СасуBP & $\mathrm{F}, \mathrm{M}, \mathrm{NMR}, \mathrm{AC}, \mathrm{GO}, \mathrm{O}$ & & 960 & \\
\hline & CacyBP/SIP & $\mathrm{AC}, \mathrm{Cl}, \mathrm{O}$ & & & Regulation of CacyBP/SP ubiquitinylation complex \\
\hline & Caldesmon & $\mathrm{F}$ & & & \\
\hline & Fetuin (biotinylated) & $\mathrm{O}$ & & & \\
\hline & GADPH & $A C, G O, F, C S, O$ & $\rightarrow \square$ विय $\square$ & 100 & \\
\hline & Нор[90] & $\mathrm{AC}, \mathrm{Cl}, \mathrm{O}$ & & & Modulation of Hsp70-Hop-90 complex formation \\
\hline & Lysozyme & GO & & & \\
\hline & Kinesin light chain (KLC & $\mathrm{Cl}, \mathrm{AC}, \mathrm{O}$ & & & Inhibition of KLC interactions with cargo proteins \\
\hline & p53 [95] & $\mathrm{F}$ & & 12000 & \\
\hline & RAGE [96] & $\mathrm{OB}, \mathrm{O}$ & & 13500 & Regulation of cell survival, proliferation and apoptosis \\
\hline & Tropomyosin & $\mathrm{F}, \mathrm{O}$ & & $0.28-1$ & Regulation of smooth muscle contraction \\
\hline & Sgt1 & $\mathrm{AC}, \mathrm{CC}, \mathrm{Cl}$ & & & Regulation of protein ubiquitination via Stg1 \\
\hline & Jab-1 [97] & $\mathrm{Y} 2 \mathrm{H}, \mathrm{Cl}$ & & & Progression of breast cancer \\
\hline S100A7 & RAGE [98] & $\mathrm{OB}$ & & & Regulation of chemotaxis \\
\hline
\end{tabular}


Table 1.1 Calcium-dependent interactions of the $\mathrm{S100}$ proteins

\begin{tabular}{|c|c|c|c|c|c|}
\hline PROTEIN & TARGET & SUPPORT $^{a}$ & REGION $^{b}$ & $\mathrm{~K}_{\mathrm{d}}(\mathrm{nM})^{\mathrm{C}}$ & FUNCTION $^{d}$ \\
\hline \multirow{5}{*}{$\begin{array}{l}\text { S100A8/ } \\
\text { S100A9 }\end{array}$} & Cytochrome b558 & 0 & & & Activation of cytochrome b558 \\
\hline & Glycoaminoglycans & O & & 6.1 & Localization of MRPs to endothelium \\
\hline & RAGE [99] & $\mathrm{Cl}, \mathrm{O}$ & & & \\
\hline & Toll-like receptor 4 [100] & $\mathrm{O}$ & & & Promotes endotoxin induced shock \\
\hline & S100A8/S100A9 (tetramer) & MS, O & & & Increasing affinity for calcium \\
\hline \multirow[t]{6}{*}{ S100A11 } & Actin & CS & & 1000 & Regulation of actin activated myosin ATPase \\
\hline & Annexin A1 & X-Ray, $\mathrm{F}, \mathrm{M}, \mathrm{CL}, \mathrm{AC}, \mathrm{Cl}$ & ¿ & 15000 & Targeting and membrane cross linking \\
\hline & Annexin A2 $[94,101]$ & NMR, O & $=$ & 3000 & Targeting and membrane trafficking \\
\hline & p53 [95] & $\mathrm{F}$ & & 61000 & \\
\hline & Rad54B [102] & $\mathrm{O}$ & & & Regulations of DNA double strand repair \\
\hline & RAGE[103, 104] & $\mathrm{OB}, \mathrm{O}$ & & & Modulation of osteoarthritis \\
\hline \multirow[t]{5}{*}{$\mathrm{S} 100 \mathrm{~A} 12$} & Annexin A5 & $A C, O B$ & & 621 & \\
\hline & CaCyBP/SIP & $A C, O$ & & & Degradation of $\alpha \beta$ catenin \\
\hline & Paramyosin & $\mathrm{E}, \mathrm{O}$ & & & Development of keratitis \\
\hline & RAGE [92] & $\mathrm{CD}, \mathrm{E}, \mathrm{OB}, \mathrm{O}$ & & & Inflammatory processes \\
\hline & S100A12 (hexamer) & X-Ray & & & Receptor signaling \\
\hline \multirow[t]{22}{*}{ S100B } & AHNAK & $\mathrm{AC}, \mathrm{Cl}, \mathrm{OB}$ & & 50 & Regulation of calcium homeostasis \\
\hline & Aldolase C & GO & & & Stimulation of aldolase $\mathrm{C}$ activity \\
\hline & Annexin A6 & $\mathrm{CL}, \mathrm{Cl}, \mathrm{F}, \mathrm{CC}$ & Jum & sub $\mu \mathrm{M}$ & Regulation of calcium flux and IF assembly \\
\hline & ATAD3A & NMR, M, MS, AC, O & & $600-800$ & Modulation of folding and localization of ATAD3A \\
\hline & CacyBP/SIP & $\mathrm{GO}, \mathrm{AC}, \mathrm{Cl}, \mathrm{F}$ & & & Regulation of ubiquitination \\
\hline & Caldesmon & $\mathrm{E}, \mathrm{F}, \mathrm{CC}$ & & 500 & Decrease in inhibition of actomyosin by caldesmon \\
\hline & CapZ (TRTK-12) & NMR, F, AC, CC, O & & $150-1000$ & Regulation of actin filament extension \\
\hline & FtsZ & $\mathrm{CL}, \mathrm{AC}$ & & & \\
\hline & GFAP & o & & & Assembly of intermediate filaments \\
\hline & Guanylate cyclase & $\mathrm{O}$ & & 2000 & Activation of guanylate cyclase \\
\hline & Intermediate filaments & $\mathrm{CC}, \mathrm{CL}, \mathrm{O}$ & & & Regulation of IF assembly and disassembly \\
\hline & $\mathrm{Hdm} 4[105]$ & $\mathrm{O}$ & & 400 & Lowering levels of p53 \\
\hline & & $\mathrm{O}$ & & 310 & Down regulation of $p 53$ \\
\hline & IQGAP1 & $\mathrm{CL}, \mathrm{Cl}, \mathrm{AC}$ & & & Membrane rearrangement \\
\hline & MAG & $\mathrm{AC}, \mathrm{CC}, \mathrm{O}$ & & 7000 & Regulation of glial cell cytoskeleton \\
\hline & Microtubules & $\mathrm{Cl}, \mathrm{O}$ & $\square \square m$ & & Regulation of MT dynamics \\
\hline & NDR & NMR, AC, O & & 500 & Modulation of NDR kinase activity \\
\hline & Neuromodulin & $\mathrm{CC}$ & & & Inhibition of neuromodulin phosphorylation by PKC \\
\hline & p53 & NMR, AC, F, OB, O & & $24-23500$ & Inhibition of p53 function \\
\hline & Phosphoglucomutase & $\mathrm{GO}, \mathrm{AC}, \mathrm{M}, \mathrm{O}$ & & & Stimulation of phosphoglucomutase activity \\
\hline & RAGE & $\mathrm{O}$ & & & Promotion of cell survival \\
\hline & Tau & $A C, C C$ & & $100-1000$ & Inhibition of tau phosphorylation by protein kinase II \\
\hline \multirow[t]{6}{*}{ S100P } & CacyBP & O & $\Omega$ & & \\
\hline & Dormant Ezrin & $A C, C L$ & & & Regulation of Ezrin ability to bind actin \\
\hline & Melittin & $\mathrm{F}, \mathrm{CD}, \mathrm{O}$ & & 5000 & \\
\hline & RAGE & $\mathrm{Cl}$ & & & Stimulation of cell proliferation and survival via RAGE \\
\hline & S100PBPR & $\mathrm{AC}, \mathrm{Cl}, \mathrm{O}$ & & & Involvement in early pancreatic cancer \\
\hline & Sgt1 & $A C$ & & & \\
\hline
\end{tabular}

a Support refers to the techniques used to study interactions. Abbreviations used: $A B=A b$ epitope mapping; $A C=A$ ffinity chromatography; $C=C$ ompetition

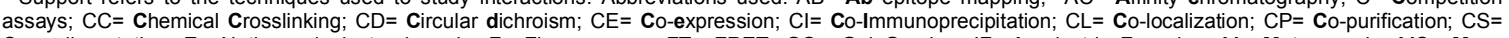
Co-sedimentation; E= Native gel electrophoresis; F= Fluorescence; FT= FRET; GO= Gel Overlay; IF= Isoelectric Focusing; M= Mutagenesis; MS= Mass spectrometry NMR= Nuclear Magnetic Resonance O= Others; OB= Optical biosensor; Ph= Phage display; $\mathrm{X}-$ Ray= Crystallography; $\mathrm{Y} 2 \mathrm{H}=\mathrm{Yeast}$ two-hybrid

${ }^{\mathrm{b}}$ The regions involved in protein interaction are shaded in black or have a heavier line in the schematic of EF-hand motifs in S100 members.

${ }^{c} \mathrm{~K}_{\mathrm{d}}$ is the dissociation constant for $\mathrm{S} 100$-target interaction. When more than one measurement was found a range of $\mathrm{K}_{\mathrm{d}}$ is reported.

${ }^{d}$ Only references reported after 2006 were added in the table. For complete references refer to Santamaria-Kisiel et al., 2006 [106]. 
A few general observations can be made from Table 1.1. Clearly, some S100 members are proposed to regulate the activity of the same target molecule. For example, S100A1, S100A6 and S100B bind annexin A6 [119-121]; S100A1, S100A2, S100A4 and S100B interact with the tumor suppressor p53 [78, 88, 122-125]; and S100B, S100A6, S100A12 and S100A1 form complexes with the ubiquitination protein CacyBP/SIP [108, 126]. This is perhaps not surprising given the significant sequence similarities of many of the S100 proteins. The apparent multi-S100 protein interactions could result from differential expression in tissues such that two different S100 proteins might control similar processes but in different tissues. It will be important to link these interactions with the expression and availability of both S100 protein and target in the same cell type and tissue. This has been elegantly shown for S100A4 and the non-muscle myosin A heavy chain implicated to have a role in metastasis [127].

For most of the S100-target protein complexes, the dissociation constant of the $\mathrm{S} 100$ member for the target is approximately $1 \mu \mathrm{M}$ (Table 1.1). This affinity is similar to that obtained for other calcium-dependent EF-hand protein complexes such as troponin-C with troponin I $(0.1-30 \mu \mathrm{M})[128,129]$. In some cases, affinity measurements may provide evidence for target selectivity amongst the S100 proteins. For example, the CapZ peptide TRTK-12 binds 10-times more tightly to S100B than S100A1, indicative of a strong preference for S100B [130, 131]. It will be important to extend these studies to off-rate measurements to determine if the release rates are consistent with the lifetimes of the biological function as recently shown for S100A4 [127]. Table 1.1 also reveals there are some disagreements regarding some S100 complexes. For example, co-sedimentation 
experiments show an interaction of F-actin with S100A4 [132], but this binding could not be observed using optical biosensor assays [124]. In the same manner, the S100A4tropomyosin (TM2) complex was observed by affinity chromatography [133] but not using surface plasmon resonance detection [124] and the binding of annexin A2 to S100A6 was reported with affinity chromatography techniques but was not detected by fluorescence or chemical crosslinking [121]. These results emphasize the need to use several methods and/or sample conditions to monitor the in vitro S100-target interactions.

Several three-dimensional structures of calcium-bound S100 proteins bound to fragments of target molecules, have been solved by either NMR or x-ray crystallography methods (Figure 1.10). The structures of S100A11 bound to the N-terminal peptide of annexin A1 [73], and S100B in complex with peptides derived from CapZ (TRTK-12) $[75,77]$, p53 [78] and NDR kinase [74] and more recently S100A6 with the C-terminal domain of Siah-1 interacting protein [76], provide atomic details about the proteinprotein interface that can be used to rationalize the specificity of the interactions. The first structure of a calcium-bound S100 protein in complex with a region of its biological target was the crystal structure of S100A11 bound to the N-terminal 14 residues of annexin A1 [73]. This structure reveals that the S100A11-annexin A1 complex remains symmetric, binding two annexin molecules per S100 dimer on the periphery of the protein. The annexin peptide lies across the linker, helix III and helix IV of one protomer and contacts helix I of the partner protomer, thus bridging the two S100 subunits (Figure 1.10). Residues near the C-terminus of helix IV in S100A11 are necessary for the annexin interaction. This region is not helical in apo-S100A11 (Figure 1.8) indicating that 
induction of an $\alpha$-helix is important for target protein binding. The annexin peptide forms an amphipathic $\alpha$-helix with its hydrophobic surface (A1, M2, V3, F6, L7, A10, W11) facing the S100 protein therefore identifying some of the amino acid determinants for the S100 specificity.

Three-dimensional structures of calcium-bound S100B with peptides derived from p53, CapZ (TRTK-12) and NDR kinase have been determined by NMR spectroscopy (Figure 1.10). These structures provide initial details for the specificity of different targets for the same S100 protein. All four of these structures utilize a similar binding region of the S100B protein, namely the surface formed by the linker and helices III and IV. The p53 and NDR targets adopt $\alpha$-helical conformations that are 12-14 residues in length. Both peptides have their $\mathrm{N}$-termini positioned towards the $\mathrm{N}$-terminus of helix III, but the orientation of the two peptides diverges by more than $8 \AA$ as they approach helix IV. Structures of calcium-bound S100B with TRTK-12 show these peptides have little regular secondary structure and are arranged roughly at $90^{\circ}$ to the orientations of the p53 or NDR peptides. These four structures point towards a malleable flat binding surface in S100B that could accommodate many different target proteins, and is shallower than that found in calmodulin [74]. This is consistent with observations in Table 1.1 showing that $\mathrm{S} 100 \mathrm{~B}$ can interact with at least 20 different proteins in a calciumdependent manner. The possibility does exist that the peptide segments being used to map S100 target interactions are void of secondary, or supplemental binding sites. Evidence for this arises from the calcium-dependent interaction of S100B with p53 where 


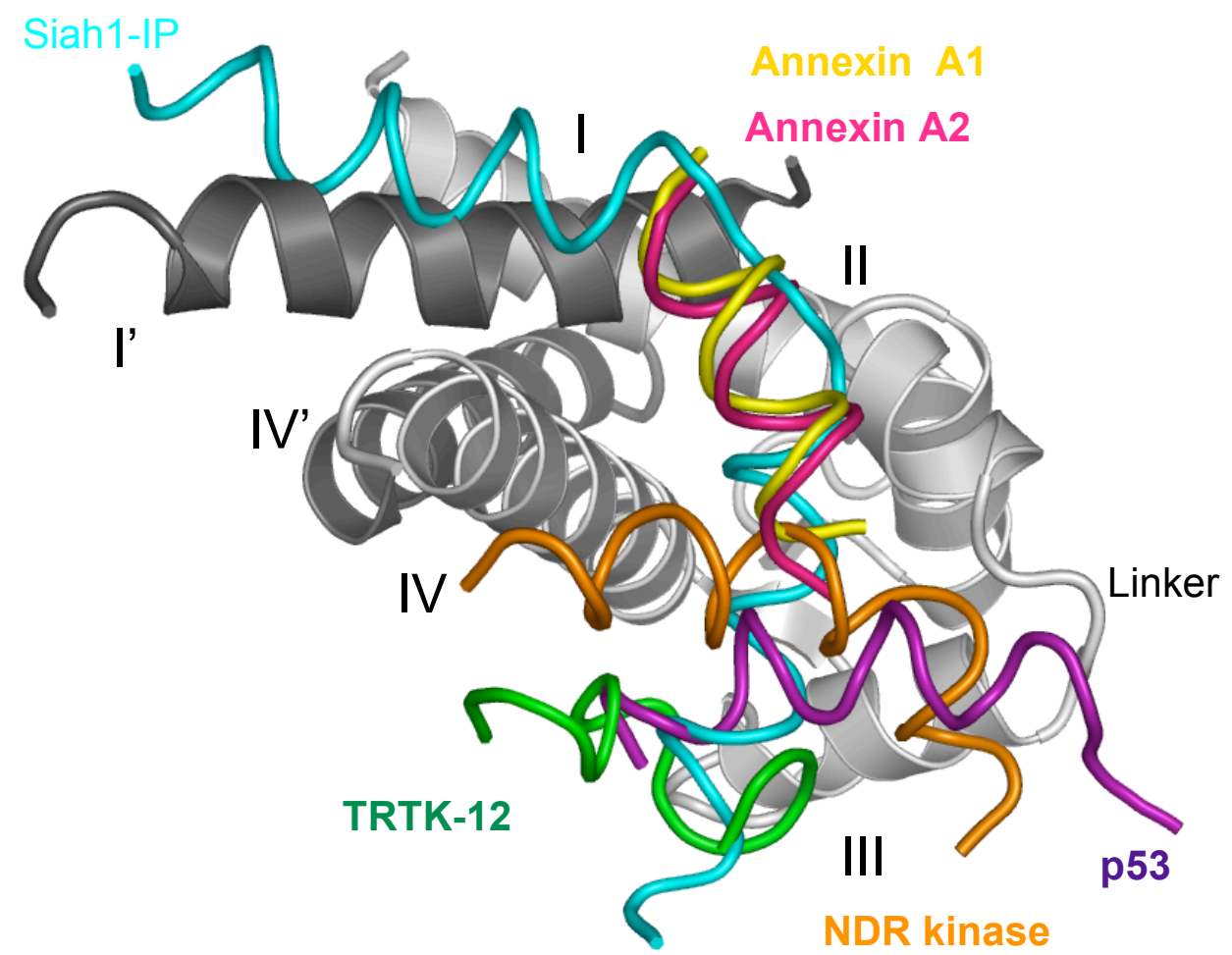

Figure 1.10 Target protein orientation for S100 proteins.

A ribbon representation of $\mathrm{Ca}^{2+}-\mathrm{S} 100 \mathrm{~A} 11$ is presented, showing one of the subunits in light grey (helices labeled as I, II, III, IV) and the other subunit is dark grey (helices labeled I' to IV'). The S100 portion from the three-dimensional complexes of S100A10annexin A2, S100B-p53, S100B-TRTK-12, S100B-NDR kinase and S100A6-Siah1 interacting protein (Siah1-IP) was superimposed with S100A11 to give relative orientation of each target peptide. This figure was drawn using MacPymol (http://delsci.com/macpymol/). 
peptides comprising residues $319-393$ possess very tight binding to $\mathrm{S} 100 \mathrm{~B}\left(\mathrm{~K}_{\mathrm{d}}=24\right.$ $\mathrm{nM}$ ) [134], but this is weakened more than 100-fold with shorter constructs (367-381 or 367-393) [122]. In order to further define the binding surfaces on the S100 proteins with specific binding partners it will be important to expand the number of structures of calcium-bound S100 family members with targets, including interactions with intact target proteins or much larger peptide sequences. Further, the precision of the structures may need to be improved, especially for side chain interactions involving the bound peptides, in order to develop better rationales for binding specificity.

The recent structure of the C-terminal domain from the Siah-1 interacting protein (SIP) with $\mathrm{Ca}^{2+}$-S100A6 [76] shows a novel mode of target interaction with the peptide binding to two different regions of the protein (Figure 1.10 in cyan). In the structure the SIP peptide (182-219) forms two non-equivalent 3-turn helices. The first of the helices utilizes the hydrophobic patch between helix III and IV, much like S100A1 and S100B complexes, while the second helix lies over the dimer interface of $\mathrm{Ca}^{2+}-\mathrm{S} 100 \mathrm{~A} 6$ bridging the two subunits, similar to the binding of annexin peptides to S100A10 and S100A11. Interesting questions result from this new mode of interaction, including the possibility of two different target molecules interacting with a single S100 protein, each occupying one of the regions described. Future structural information including longer peptides or fulllength targets will provide insi3ghts into the specificity of the regions preferred for S100target interactions. All of the calcium-bound S100A11 and S100B complexes utilize helix IV for their target protein interaction. The TRTK-12 and p53 sequences interact with regions near V80-C84, while the NDR peptide interacts mostly downstream of this (C84- 
F88). In contrast, annexin A1 utilizes the opposite face of helix IV in S100A11 and SIP uses both regions in S100A6. This shows the central adaptive role that helix IV plays to modify S100 target protein interactions. Examination of Table 1.1 corroborates this idea as more than 25 calcium-dependent binding partners with different S100 proteins utilize helix IV or the subsequent C-terminus. For example, deletion of the S100A1 C-terminus abolishes its interaction with TRTK-12, GFAP, p53, SERCA2a and PBL [135]. However, not all S100 target proteins follow this trend. For instance, the N-terminal region of S100A6 is essential for its interaction with GADPH and Annexin A2 [136]. These observations and the variations in the three-dimensional structures of calcium-bound S100-target complexes provide some insight into the level of target specificity among the S100 family members. It is clear that although several target proteins appear to "cross react" with more than one S100 protein and most S100 proteins interact with several targets, there remains a fine level of discrimination that does not allow random interaction of targets with all S100 proteins. The broad selection of the S100 targets such as TRTK-12, NDR and p53 is likely a reflection of the moderate hydrophobicity in the short target peptide sequence and the extensive hydrophobic patch that exists on the S100 structures. Nevertheless, this exposed region is comprised of most divergent portions of the S100 sequences (the linker and C-terminal regions) and this must be enough to discriminate against different target proteins.

\subsubsection{Calcium independent protein interactions}

Although the majority of S100 protein interactions are calcium dependent, several calcium independent interactions have been reported (Table 1.2). The most common 
binding partners for the apo-S100 proteins are enzymes. For example S100B and S100A1 bind with glycogen phosphorylase [137] while S100A10 and S100A11 show interactions with transglutaminase [138]. Some discrepancies for the calcium sensitivity of S100-target interactions are encountered. For example, optical biosensor techniques show a calcium insensitive complex between S100A4 and non-muscle myosin (heavy chain II, isoform A), and between S100A4 and p53 [124], while strict calcium dependence was observed by other techniques [124, 127, 132, 140-144]. In some cases such as the calcium insensitive interaction of S100A4 with F-actin the dissociation constant is very weak $\left(\mathrm{K}_{\mathrm{d}} \sim 500 \mu \mathrm{M}\right)$ but strengthens significantly upon calcium binding to the $\mathrm{S} 100$ protein $\left(\mathrm{K}_{\mathrm{d}} \sim 30 \mu \mathrm{M}\right)$ [132]. Similar observations have been noted for the interaction of melittin with S100P [145]. These poor binding affinities in the absence of calcium likely indicate the in vivo interaction is insignificant in the absence of calcium.

The most important calcium independent interactions of the S100 proteins are their abilities to form homo- and heterodimers, as well as some higher order complexes (Table 1.3). This property results in a dynamic interplay between formation of the dimeric species, calcium binding and interactions with a biological target protein (Figure 1.1). Traditionally, homodimeric interactions have been the focus of the S100 proteins. However the use of yeast two-hybrid experiments has uncovered a large number of heterodimer complexes.

Further, optical biosensor experiments have been used to quantify the strength of the homo- and heterodimeric interactions in vitro and so that the importance of these can be assessed in vivo. 
Table 1.2 Calcium-independent interactions of the S100 proteins.

\begin{tabular}{|c|c|c|c|c|c|}
\hline PROTEIN & TARGET & SUPPORT $^{\mathrm{a}}$ & REGION $^{b}$ & $K_{d}(n M)^{c}$ & FUNCTION $^{d}$ \\
\hline \multirow[t]{4}{*}{ S100A1 } & Adenylate cyclase & $\mathrm{O}$ & & 200 & Stimulation of adenylate cyclase \\
\hline & Aldolase A & $\mathrm{O}$ & & & \\
\hline & Glycogen phosphorylase & $\mathrm{GO}, \mathrm{AC}, \mathrm{CL}$ & & & Inhibition of glycogen phosphorylase activity \\
\hline & Microtubules & $\mathrm{O}$ & & & Microtubule assembly \\
\hline \multirow[t]{6}{*}{ S100A4 } & Annexin A2 & $\mathrm{Cl}, \mathrm{NMR}, \mathrm{O}$ & & & Mediation of plasmin production from plasminogen \\
\hline & F-actin & OB & & 543000 & \\
\hline & Liprin $\beta 1$ & $\mathrm{Cl}, \mathrm{CL}, \mathrm{MS}, \mathrm{O}$ & & & Inhibition of Liprin $\beta 1$ phosphorylation \\
\hline & Myosin heavy chain II -A & $\mathrm{OB}$ & $\neg \curvearrowleft$ & $220-600$ & Regulation of cytoskeletal dynamics \\
\hline & p53 & $\mathrm{OB}$ & & $8.6-14$ & Enhancement of p53-dependent apoptosis in tumors \\
\hline & S100A4 (tetramer) & MS & & & Stimulation of angiogenesis and neurite growth \\
\hline \multirow[t]{4}{*}{ S100A7 } & E-FABP & $\mathrm{CL}, \mathrm{Cl}, \mathrm{CP}, \mathrm{GO}$ & & & Shuttle E-FABP to membrane or ligands \\
\hline & jab-1 & $\mathrm{Y} 2 \mathrm{H}, \mathrm{Cl}, \mathrm{O}$ & $\rightarrow \square m$ & & Pro-survival pathway \\
\hline & RanBPM & $\mathrm{Y} 2 \mathrm{H}, \mathrm{Cl}$ & & & Cytoskeletal functions, adhesion and migration \\
\hline & transglutaminase & $\mathrm{O}$ & $\rightarrow \Omega$ 口и & & Regulation of S100A7 function \\
\hline S100A8/ & Carboxylated glycans & $\mathrm{AC}$ & & & Inflammation \\
\hline \multirow{2}{*}{ S100A9 } & CD36 & $A C$ & & & Fatty acid uptaking \\
\hline & Phox proteins & $\mathrm{CP}, \mathrm{Cl}$ & & & Scaffold for phox and NADPH oxidase activation \\
\hline \multirow[t]{12}{*}{ S100A10 } & $5-\mathrm{HT}_{1 \mathrm{~B}}$ receptor & $\mathrm{Y} 2 \mathrm{H}, \mathrm{Cl}, \mathrm{CL}, \mathrm{O}$ & & & Localization of $5-\mathrm{HT}_{1 \mathrm{~B}}$ receptors to the cell surface \\
\hline & ASIC1a[139] & $\mathrm{Y} 2 \mathrm{H}, \mathrm{Cl}$ & & & Enhancement of cell surface expression of ASIC1a \\
\hline & Annexin A2 & X-Ray, CD, F, M, CC, CL, O & $\rightarrow-\Omega$ & $\sim 30$ & Signal transduction \\
\hline & NS3 & $\mathrm{Y} 2 \mathrm{H}$ & & & Mediation of virus release \\
\hline & Connexin 31 & $\mathrm{Y} 2 \mathrm{H}$ & & & \\
\hline & Phospholipase A2 & $\mathrm{Cl}, \mathrm{O}$ & & & Regulation of PLA2 activation \\
\hline & HBV Pol & $\mathrm{Y} 2 \mathrm{H}, \mathrm{AC}, \mathrm{Cl}, \mathrm{CL}, \mathrm{O}$ & & & Inhibition of the DNA polymerase activity of HBV Pol \\
\hline & TASK-1 $\mathrm{K}^{+}$channel & $\mathrm{Y} 2 \mathrm{H}, \mathrm{AC}, \mathrm{Cl}, \mathrm{M}, \mathrm{O}$ & & & Trafficking of TASK- 1 to the plasma membrane \\
\hline & Plasminogen activator & $\mathrm{AC}, \mathrm{O}$ & $\rightarrow \square \square$ & & \\
\hline & Sodium channel $\mathrm{Na}_{\mathrm{v}} 1.8$ & $\mathrm{Y} 2 \mathrm{H}, \mathrm{AC}, \mathrm{O}$ & $\rightarrow \square \square$ сиц & & Translocation of $\mathrm{Na}_{v} 1.8$ to the plasma membrane \\
\hline & Transglutaminase & $\mathrm{O}$ & $\rightarrow \square \square \operatorname{man} \square-$ & & Regulation of S100A10 function \\
\hline & TRPV5/TRPV6 & $\mathrm{Y} 2 \mathrm{H}, \mathrm{AC}, \mathrm{Cl}$ & $\rightarrow \curvearrowleft \square$ mи $\square$ & & Translocation TRPV5/TRPV6 to plasma membranes \\
\hline \multirow[t]{4}{*}{ S100A11 } & Transglutaminase & $\mathrm{O}$ & & & Regulation of S100A11 function \\
\hline & Isocitrate dehydrogenase & $\mathrm{AC}, \mathrm{OB}$ & & & \\
\hline & Aldolase A & & & & \\
\hline & GAPDH & $\mathrm{AC}, \mathrm{OB}$ & & 82.7 & \\
\hline \multirow[t]{2}{*}{$\mathrm{S} 100 \mathrm{~A} 13$} & FGF-1, p40 Syt-1 & $\mathrm{CP}, \mathrm{E}, \mathrm{CL}, \mathrm{O}$ & & & Regulation of FGF-1 release \\
\hline & IL-1alpha & $\mathrm{CL}, \mathrm{Cl}$ & $\rightarrow \square \square$ mи $\square$ & & Stress induced release of IL-1alpha \\
\hline \multirow[t]{2}{*}{ S100B } & Aldolase A & $\mathrm{O}$ & & & Stimulation of aldolase $A$ activity \\
\hline & Glycogen phosphorylase & $\mathrm{GO}, \mathrm{AC}$ & & & \\
\hline S100P & Melittin & $\mathrm{F}, \mathrm{CD}, \mathrm{O}$ & & 200000 & \\
\hline
\end{tabular}

${ }^{a}$ Support refers to the techniques used to study interactions. Abbreviations used: $A B=A b$ epitope mapping; $A C=A$ ffinity chromatography; $C=C o m p e t i t i o n$

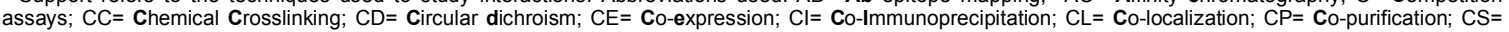
Co-sedimentation; E= Native gel electrophoresis; F= Fluorescence; FT= FRET; GO= Gel Overlay; IF= Isoelectric Focusing; M= Mutagenesis; MS= Mass spectrometry NMR= Nuclear Magnetic Resonance O= Others; OB= Optical biosensor; Ph= Phage display; X-Ray= Crystallography; $\mathrm{Y} 2 \mathrm{H}=\mathrm{Yeast}$ two-hybrid

${ }^{\mathrm{b}}$ The regions involved in protein interaction are shaded in black or have a heavier line in the schematic of EF-hand motifs in S100 members.

${ }^{c} \mathrm{~K}_{d}$ is the dissociation constant for $\mathrm{S} 100$-target interaction. When more than one measurement was found a range of $\mathrm{K}_{d}$ is reported.

${ }^{d}$ For complete references refer to Santamaria-Kisiel, L. et al., 2006. [106] 
The association of several homodimeric S100 proteins has been quantified providing an indication of the overall stability of S100B, S100A4, S100A12 and S100P. These studies show a wide range of dissociation constants for the protomer-dimer equilibrium. For example, S100B forms the tightest dimer $\left(\mathrm{K}_{\mathrm{d}}<500 \mathrm{pM}\right)$ in the calciumfree state [146] and remarkably is nearly 5000-fold more stable than either the S100A4 or S100P homodimers $\left(\mathrm{K}_{\mathrm{d}} \sim 1-2 \mathrm{mM}\right)$. In some cases (S100P and S100A12), dimerization is enhanced by more than 100-fold in the presence of calcium. In other cases (S100B, S100A4) calcium binding has a negligible effect. In vivo, the extent of homodimerization will be dependent on the concentration of the S100 protein and the dissociation constant for dimer formation. For example, S100B has been found at high concentrations (nearly $10 \mu \mathrm{M})$ in glial cells [147], indicating that this protein would be completely in the dimeric form. On the contrary, proteins such as S100A4 and S100P, if found in similar or lower concentrations than S100B would have a significant population of monomeric protein in the cell. This would facilitate formation of heterodimers with other S100 proteins. The observation that dynamic exchange occurs for the S100 subunits indicates that heterodimeric proteins likely exist in vivo. Consistent with this, at least 10 different heterodimeric S100 species have been identified. The S100A8/S100A9 heterodimer is probably the best characterized of these. Originally isolated from synovial fluid [148], Xray structures are available for the S100A8 and S100A9 homodimers [68, 149] However, other experiments have shown there is a strong preference for the S100A8/S100A9 heterodimer, especially in the presence of calcium and it has been suggested that the S100A8/S100A9 heterodimeric species is the only relevant biological 
Table 1.3 Homo- and heterodimer formation of the S100 proteins.

\begin{tabular}{|c|c|c|c|c|}
\hline PROTOMER I & PROTOMER II & SUPPORT $^{\mathrm{a}}$ & REGION $^{\mathrm{b}}$ & FUNCTION $^{d}$ \\
\hline \multirow[t]{3}{*}{ S100A1 } & S100A4 & $\begin{array}{l}\text { Y2H, F, FT, MS, OB, } \\
\mathrm{AC}, \mathrm{GO}, \mathrm{CP}, \mathrm{CE}, \mathrm{M}\end{array}$ & $\neg \square \square \operatorname{man} \square$ 300-500 (+Ca) & Modulation of metastasis in cancer cells \\
\hline & S100B & $\mathrm{Y} 2 \mathrm{H}, \mathrm{O}$ & & \\
\hline & S100P & $\mathrm{Y} 2 \mathrm{H}, \mathrm{OB}, \mathrm{FT}, \mathrm{CE}, \mathrm{M}$ & $\square \square \square-\begin{array}{c}1 \\
100-1800(\mathrm{apo}) \\
10-20(+\mathrm{Ca})\end{array}$ & Target binding and function regulation \\
\hline S100A4 & S100A4 & Y2H, OB, NMR, O & $\neg \square \square \begin{array}{c}4000(\mathrm{apo}) \\
670-1000 \text { (+Ca) }\end{array}$ & \\
\hline S100A6 & S100B & $\mathrm{Y} 2 \mathrm{H}, \mathrm{Cl}, \mathrm{CL}$ & & Possible role in control of melanoma cell growth \\
\hline S100A7 & S100A10 & $\mathrm{MS}, \mathrm{Cl}, \mathrm{O}$ & & \\
\hline \multirow[t]{2}{*}{ S100A8 } & S100A9 & $\begin{array}{l}\text { Y2H, MS, AC, M, Ph, } \\
\text { AB, CC, CD, F, NMR, } \\
\text { XRay, E, IF }\end{array}$ & $\neg \square \square$ man & Inflammatory processes \\
\hline & S100A10 & $\mathrm{MS}, \mathrm{Cl}, \mathrm{O}$ & & \\
\hline S100A9 & S100A12 & $\mathrm{AC}, \mathrm{Cl}, \mathrm{OB}, \mathrm{M}, \mathrm{O}$ & $\neg \square$ man $\square \Omega$ & \\
\hline S100A11 & S100B & $\mathrm{Y} 2 \mathrm{H}, \mathrm{CL}, \mathrm{Cl}$ & $\neg \curvearrowleft \square$ пи $\square$ - & Modulation of target binding \\
\hline S100A12 & S100A12 & Xray, OB & $4(+\mathrm{Ca})$ & \\
\hline$\overline{\mathrm{S} 100 \mathrm{~B}}$ & S100B & NMR, O & $\begin{array}{l}<500 \mathrm{pM}(\text { apo }) \\
<500 \mathrm{pM}(+\mathrm{Ca})\end{array}$ & \\
\hline \multirow[t]{2}{*}{ S100P } & S100P & $\mathrm{Y} 2 \mathrm{H}, \mathrm{Xray}, \mathrm{OB}$ & $\begin{array}{c}1400-2500 \text { (apo) } \\
40-120(+\mathrm{Ca})\end{array}$ & Involved in various diseases \\
\hline & S100Z & $\mathrm{Y} 2 \mathrm{H}, \mathrm{IF}, \mathrm{E}$ & & \\
\hline
\end{tabular}

a Support refers to the techniques used to study interactions. Abbreviations used: $A B=A b$ epitope mapping; $A C=A$ ffinity chromatography; $C=C$ Competition assays; $\mathrm{CC}=$ Chemical Crosslinking; $\mathrm{CD}=$ Circular dichroism; $\mathrm{CE}=\mathrm{Co}$-expression; $\mathrm{Cl}=\mathrm{Co}$-Immunoprecipitation; $\mathrm{CL}=\mathrm{Co}-\mathrm{localization} ; \mathrm{CP}=\mathrm{Co}-\mathrm{purification} ; \mathrm{CS}=$ spectrometry NMR= Nuclear Magnetic Resonance O= Others; OB= Optical biosensor; $\mathrm{Ph}=$ Phage display; $\mathrm{X}$-Ray= Crystallography; $\mathrm{Y} 2 \mathrm{H}=\mathrm{Yeast}$ two-hybrid

${ }^{\mathrm{b}}$ The regions involved in protein interaction are shaded in black or have a heavier line in the schematic of $\mathrm{EF}$-hand motifs in $\mathrm{S} 100$ members ( $\mathrm{N}$-terminus-HelixlSite1-HelixII-Linker-HelixIII-Site2-HelixIV-C-terminus)

${ }^{\mathrm{c}}$ When more than one measurement was found a range of $\mathrm{K}_{d}$ is reported.

${ }^{\mathrm{d}}$ For complete references refer to Santamaria-Kisiel, L. et al., 2006. [106] 
species [150]. A similar calcium stabilization of the heterodimer has been observed for S100B/S100A6 and S100B/S100A11. In the case of the other S100 heterodimers there are several intriguing possibilities. The first of course is that the relevant S100 proteins must be found in the same cell type to substantiate heterodimer formation. This has been confirmed for several species including S100A1/S100A4 found in several mammary cell lines and S100B/S100A6 found in some human melanoma cells [151, 152].

Further, the formation of the S100 heterodimeric proteins will be governed by the thermodynamics of the equilibria involved, shown previously for homo- and heterodimeric calcium-binding peptides [153], and for tropomyosin [154]. Using the example of S100A1 and S100A4, the S100A1/S100A4 heterodimer will be preferentially formed when $\Delta \mathrm{G}_{\mathrm{S} 100 \mathrm{~A} 1 / \mathrm{S} 100 \mathrm{~A} 4}<1 / 2\left[\Delta \mathrm{G}_{\mathrm{S} 100 \mathrm{~A} 1}+\Delta \mathrm{G}_{\mathrm{S} 100 \mathrm{~A} 4}\right]+\mathrm{RT} \ln 2$. Based on Table 1.3 , the stability of S100A1/S100A4 $\left(\mathrm{K}_{\mathrm{d}} \sim 300 \mathrm{nM}\right)$ is nearly 10 -fold that of the S100A4 homodimer $\left(\mathrm{K}_{\mathrm{d}} \sim 1-2 \mu \mathrm{M}\right)$ judged by yeast two-hybrid, optical biosensor and analytical ultracentrifugation experiments $[151,155,156]$. Further, yeast two-hybrid studies have shown the S100A1 homodimer likely has a similar stability as the S100A1/S100A4 heterodimer. Based on these observations and the above inequality, the S100A1/S100A4 heterodimer would be favoured thermodynamically by approximately $3 \mathrm{~kJ} / \mathrm{mol}$. More complicated situations may arise in the cell including interactions with other S100 proteins or binding partners, or large differences in cellular concentrations of the S100 protein. However, the thermodynamic point of view would indicate the S100A1/S100A4 heterodimer is the dominant in vivo species. For other heterodimers, this approach indicates the homodimer is the major species. For example, the extremely tight dimer 
association of S100B indicates that heterodimers such as S100B/S100A11 and S100B/S100A6 would be poorly formed in vivo. Although dissociation constants are not available for these two heterodimers, two-hybrid experiments show approximately a 2fold poorer $\beta$-galactosidase activity for the heterodimers $[152,157]$. Further, experiments will be needed for other S100 homo- and heterodimers to establish their strengths of interaction and relative populations in different cell types.

Some important differences regarding the specificity of interaction at the dimer interface have been noted for S100 homo- and heterodimers using site-directed mutagenesis and yeast-two-hybrid experiments. For example, deletion of the C-terminal 8 residues for S100B abolishes heterodimer formation with both S100A6 and S100A11, while S100B homodimer formation is unaffected [157]. Within this region, residues F87 and F88 are particularly important. These residues are in an unstructured region following helix IV and have very few intersubunit contacts in the apo-S100B structure. In order to contribute substantially to the heterodimer complex these interactions must be significantly altered in the heterodimer structures. Similar observations have been made for S100A1/S100A4 and S100A1/S100P where C76 and C81 in S100A4 [158], and V76 in S100P [159] are required for heterodimerization with S100A1, but have little affect on the respective homodimer formation.

\subsection{Biology of S100A10}

S100A10 (p11, annexin 2 light chain) is a calcium insensitive member of the S100 family since its two EF-hand loops carry substitutions in residues involved in coordination of the ion. Despite this deficiency S100A10 in the absence of calcium 
adopts a conformation resembling other $\mathrm{Ca}^{2+}$-bound $\mathrm{S} 100$ proteins, particularly that of $\mathrm{Ca}^{2+}-\mathrm{S} 100 \mathrm{~A} 11$ (Figure 1.11). Thus, S100A10 forms tight complexes with a variety of proteins in vivo (Table 1.2) and responds well to two-hybrid, co-immunoprecipitation and other in vivo methods to identify target proteins. To date, S100A10 is the only S100 protein to display this property.

S100A10 was first identified as a heterotetramer complex with the cytosolic protein annexin A2 [160]. Annexin A2 is a member of a different class of $\mathrm{Ca}^{2+}$-binding proteins that interact with phospholipids in a calcium-dependent manner. More recently, an important interaction between $\mathrm{S} 100 \mathrm{~A} 10$ and the serotonin receptor $5-\mathrm{HT}_{1 \mathrm{~B}}$ has been identified using a yeast two-hybrid screen. Further experiments have provided strong evidence that this S100A10-serotonin complex may have a role in the onset of depression [161]. Other complexes with S100A10, including those with the viral proteins NS3 and hepatitis B polymerase, have been identified using yeast two-hybrid assays [162, 163] (Table 1.2), as these protein interactions are calcium insensitive [160]. The S100A10annexin A2 complex was the first three-dimensional structure of an S100 complex to be determined [57]. As with S100A11, the S100A10 protein interacts with the N-terminus of the annexin molecule. The fold of the S100A10 protein is nearly identical to that of calcium-bound S100A11 (Figure 1.11), adopting the more "open" conformation that provides a hydrophobic surface for protein binding, even in the absence of calcium. The location and interactions of the annexin A2 peptide with apo- S100A10 are remarkably similar to those identified for S100A11 (rmsd $0.87 \AA$ ) [73], interacting with residues from helices III and IV, and the linker of one protomer, and helix I of the other subunit 
(Figure 1.11). Other protein partners, including tissue-type plasminogen activator appear to utilize the same helix IV region (Table 1.2) while targets such as the Na-channel (V)1.8 interact at different sites [164]. S100A10 is found in different cells and tissues with the highest levels of expression observed in the lung, intestine and kidney and it is believed to be involved in exocytosis and endocytosis [165].

New information has been reported regarding biological roles of S100A10 and its diverse interacting partners. For example, yeast two hybrid experiments showed that S100A10 is able to interact with the Acid-Sensing Ion Channel (ASIC1a) [139]. ASIC1a are ion channels capable of transporting $\mathrm{Na}^{+}$through the cell membrane upon activation by extracellular protons. S100A10 seems to be involved in enhancing ASIC1a functional expression. In addition, S100A10 is also involved in trafficking the Transient Receptor Potential Channel (TRPV5 and TRPV6) to the plasma membrane and co-localization studies provided evidence for an important modulation of the serotonin 1B receptor by S100A10, which is associated with depression-like states [161]. Further, S100A10 knockout mice experiments have shown the mice are viable indicating that S100A10 is not essential for normal development. However, the mice presented a depression-like phenotype and reduced sensitivity to serotonin $1 \mathrm{~B}$ receptor agonists and lower response to anti-depressants. This suggests a main role for $\mathrm{S} 100 \mathrm{~A} 10$ in regulating 5 -HT1B receptor function and subsequent depressive disorders [161].

\subsection{Biology of S100A11}

S100A11 (S100C, Calgizzarin) is a homodimer of $22 \mathrm{kDa}$ (101 residues per subunit). Each protomer contains high-affinity (Site II) and low-affinity (Site I) calcium- 


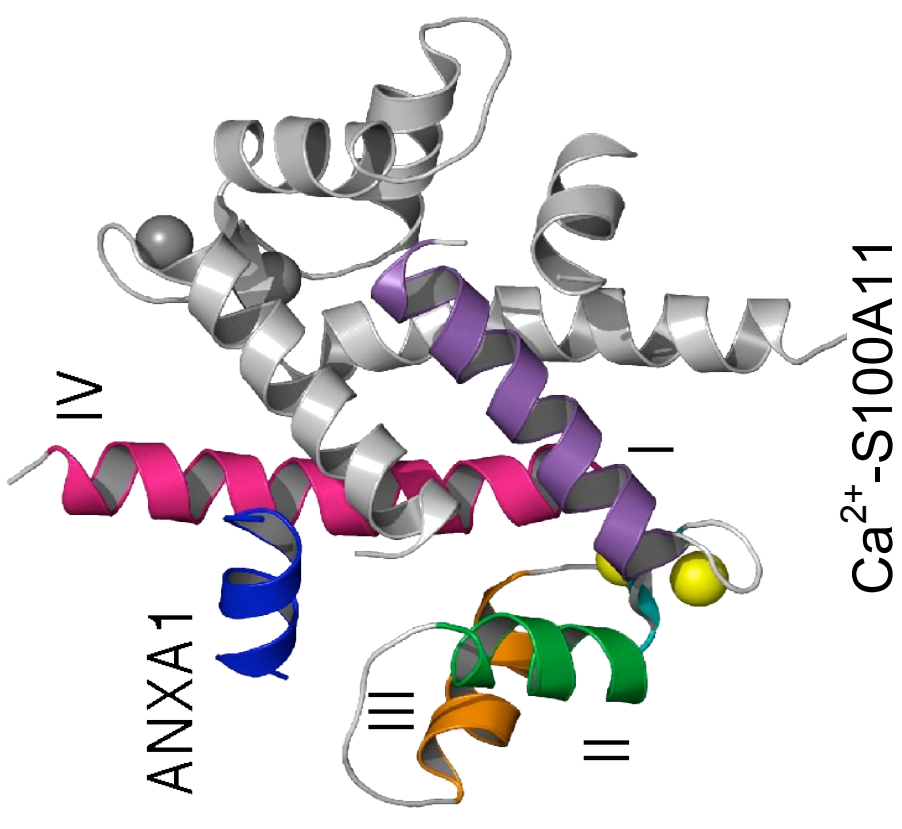

可过导章

ㄷ. 중

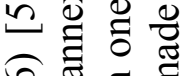

6 ․․

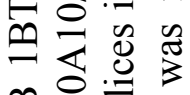

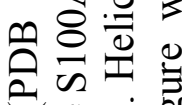

월

$\ll 4 \infty \cdot$

:

节导㤩

पै के

.를 웡

क्ष क

든

矛安.

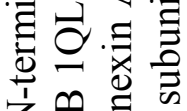

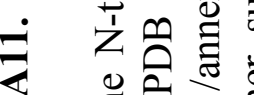
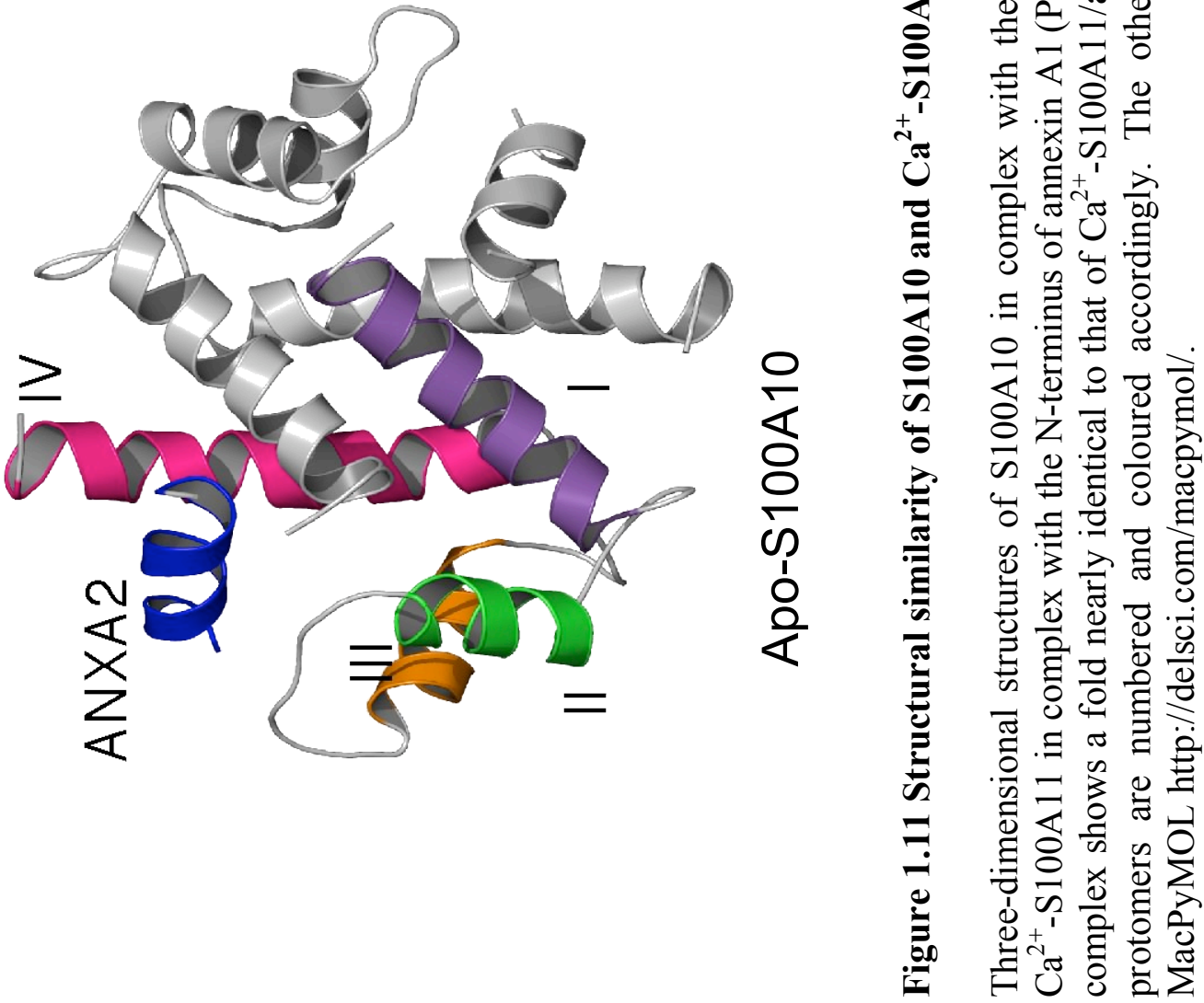
S100A11 was first purified from chicken gizzard (hence the name Calgizzarin) [166] but is also expressed in smooth muscle containing tissues such as heart, lung and placenta [166, 167]. S100A11 binds with Rad54B in a calcium-dependent manner, suggesting a possible role in DNA double strand repair mechanism and cell cycle progression [102]. Moreover, the $\mathrm{Ca}^{2+}$ and phospholipid proteins annexins A1, A2 and A6 have been suggested as physiological targets for S100A11 [73, 101, 168]. S100A11 can inhibit the phosphorylation at the annexin A1 N-terminus modulating its interaction with lipids in a calcium dependent manner. Due to the calcium-dependent interaction of S100A11 with annexin proteins a regulatory function involving recruitment of other cytoskeletal proteins to the cell membrane has been implicated. One possibility is that S100A11 functions in membrane repair mechanism and vesiculation (endo/exocytosis), through reversible $\mathrm{Ca}^{2+}$-dependent binding to the annexin proteins [169].

S100A11 is also a key mediator for growth inhibition of normal epidermal keratinocytes (NHK) triggered by high $\mathrm{Ca}^{2+}$ or TGF- $\beta$. Phosphorylation of S100A11 by protein kinase $\mathrm{C}(\mathrm{PKC} \alpha)$ links these two pathways [170]. Exposure of the cell to high concentrations of calcium or TGF- $\beta$ results in phosphorylation of Thr10 allowing binding and transferring to the nucleus [171]. Recently, the receptor for advanced glycation end products (RAGE) has been identified as a target for S100A11, proposing a role for the S100 protein in the regulation of RAGE activation [103].

\subsection{Scope of the thesis}

Structural studies of the S100 proteins in their calcium-free and bound states have significantly contributed to our understanding of their activation, and in mediating 
interactions with downstream effector proteins. Indeed, identification of over 90 calcium-dependent S100-complexes has provided insights into their potential physiological roles, and underscores their importance in calcium signaling. Since many of these interactions have been identified using in vitro approaches, they remain controversial since co-localization or complex formation in vivo cannot be demonstrated.. This occurs because the resting intracellular calcium levels $(\sim 100 \mathrm{nM})$ are too low to allow binding of the ion (and subsequent conformational change) and transient increases of calcium are too short to maintain and identify the S100-target interaction [172].

Unlike most of the S100 proteins, strong in vivo evidence has been reported for S100A10-target interactions, including those with annexin A2 and most recently the Acid-Sensing Ion Channel [139]. S100A10 does not bind calcium and maintains the “open' conformation exhibited by other S100 proteins in their calcium-bound states. This constitutively active form of S100A10 has allowed biological roles in membrane trafficking, repair and vesicle assembly to be clearly identified. It follows that other S100 proteins, if designed to form the "open" conformation might also interact with in vivo targets in the absence of calcium. The aim of this thesis is to identify the regions of S100A10 that allow it to adopt a permanently "open" conformation so as to provide a blueprint for the design of other calcium-insensitive S100 proteins that can be used in the identification of in vivo targets at resting calcium levels.

The goals of this thesis were: (1) use structural and sequence information to initially identify potential regions on S100A10 that could contribute to its open conformation, (2) design and synthesize a series of chimaeric proteins proposed to have 
the open conformation, (3) use a variety of biochemical methods to examine the properties of the chimaeric proteins.

The systematic approach described in this thesis had three steps (Figure 1.12). The first step involved detailed structural analysis of S100A11 and S100A10 in the calcium-free and calcium-bound forms. In particular, S100A11 was chosen as a template because (a) the conformation $\mathrm{Ca}^{2+}-\mathrm{S} 100 \mathrm{~A} 11$ strongly resembles that of S100A10 of (rmsd $0.87 \AA$ ) (Figure 1.11), (b) both proteins share at least one identical target (annexin A2) with similar binding sites (Figure 1.11) and (c) the two proteins share $41 \%$ sequence identity (Figure 1.13). Analyses included a combination of sequence alignments; $\alpha$ carbon distances measurements to analyze the backbone folding of the proteins, interresidue contact analyses to identify unique interactions in S100A10, and hydrophobic surface area exposure comparisons. The second stage used this information to design seven chimaeric proteins where regions of calcium binding loops or helical sections in S100A10 were incorporated into S100A11. This involved using site directed mutagenesis to incorporate sequences of S100A10 into the S100A11 template, and developing methods of expression and purification for the chimeras. Finally, the chimaeric S100A11 proteins were characterized in terms of (1) increased surface area of hydrophobic residues using a hydrophobic probe (ANS) and phenyl Sepharose matrix; (2) calcium-insensitive target binding towards annexin A2 and [173] altered structure using NMR spectroscopy. These experiments provide the first steps towards designing a constitutively active S100 protein that might be used to identify in vivo target proteins and subsequently elucidate the biological roles of the S100 proteins. 


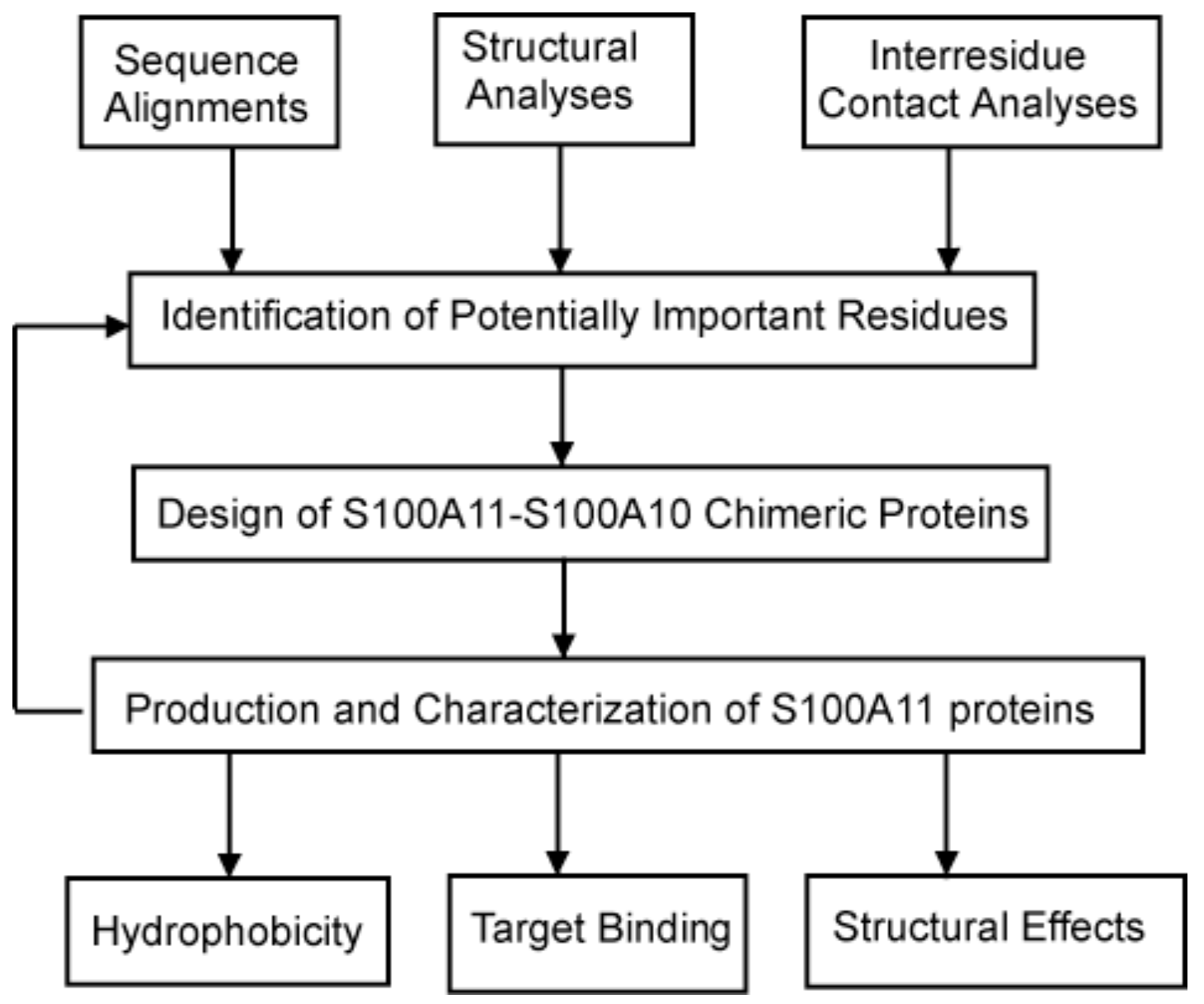

Figure 1.12 Iterative process to design S100A11/S100A10 chimaeric proteins.

Comparative analyses of S100A11 and S100A10 sequences, structures and amino acids interactions are used to identify residues that could be responsible for the permanently open form of S100A10. The chimaeric proteins designed are evaluated in terms of increased exposure of hydrophobic surfaces, target binding in the absence of calcium and conformational changes respect to apo-S100A11. 

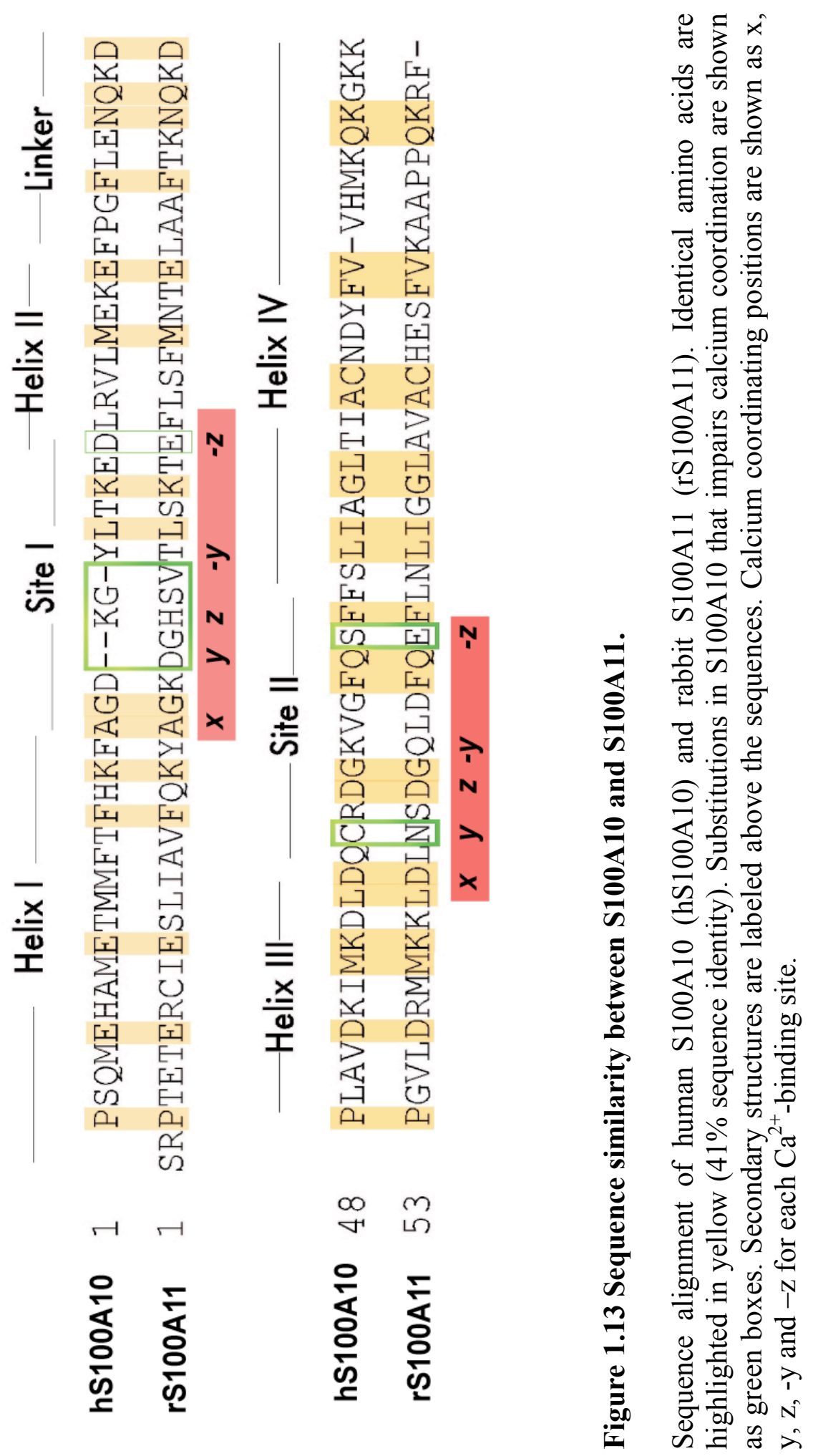


\subsection{References}

1 Vogel, H. J. (1994) Calmodulin: A versatile calcium mediator protein. Biochem. Cell Biol. 72, 357-376

2 Berridge, M. J. (1993) Inositol triphosphate and calcium signalling. Nature. 361, 315-325

3 Clapham, D. E. (1995) Calcium signaling. Cell. 80, 259-268

4 Berridge, M. J., Bootman, M. D. and Roderick, H. L. (2003) Calcium signalling: dynamics, homeostasis and remodelling. Nat Rev Mol Cell Biol. 4, 517-529

5 Henikoff, S. (2007) ENCODE and our very busy genome. Nat Genet. 39, 817-818

6 Kretsinger, R. H. and Nockolds, C. E. (1973) Carp muscle calcium-binding protein. II. Structure determination and general description. J. Biol. Chem. 248, 3313-3326

7 Strynadka, N. C. J. and James, M. N. G. (1989) Crystal structures of the helix-loophelix calcium-binding proteins. Ann. Rev. Biochem. 58, 951-998

8 Marsden, B. J., Shaw, G. S. and Sykes, B. D. (1989) Calcium binding proteins. Elucidating the contributions to calcium affinity from analysis of species variants and peptide fragments. Biochem. Cell Biol. 68, 587-601

9 Shaw, G. S., Hodges, R. S. and Sykes, B. D. (1990) Calcium-induced peptide association to form an intact protein domain: ${ }^{1} \mathrm{H}$ NMR structural evidence. Science. 249, 280-283

10 Herzberg, O., Moult, J. and James, M. N. G. (1986) A model for the $\mathrm{Ca}^{2+}$-induced conformational transition of troponin C. J. Biol. Chem. 261, 2638-2644

11 Ikura, M., Barbato, G., Klee, C. B. and Bax, A. (1992) Solution structure of calmodulin and its complex with a myosin light chain kinase fragment. Cell Calcium. 13, 391-400

12 Smith, S. P. and Shaw, G. S. (1998) A novel calcium-sensitive switch revealed by the structure of human S100B in the calcium-bound form. Structure. 6, 211-222

13 da Silva, A. C. R. and Reinach, F. C. (1991) Calcium binding induces conformational changes in muscle regulatory proteins. Trends Biochem. Sci. 16, 5357

14 Skelton, N. J., Akke, M., Kordel, J., Thulin, E., Forsen, S. and Chazin, W. J. (1992) ${ }^{15} \mathrm{~N}$ NMR assignment and chemical shift analysis of uniformly labeled ${ }^{15} \mathrm{~N}$ calbindin $\mathrm{D}_{9 \mathrm{k}}$ in the apo, $\left(\mathrm{Cd}^{2+}\right)_{1}$ and $\left(\mathrm{Ca}^{2+}\right)_{2}$ states. FEBS Lett. 303, 136-140

15 Szebenyi, D. M. E. and Moffat, K. J. (1986) The refined structure of vitamin Ddependent calcium-binding protein from bovine intestine. J. Biol. Chem. 261, 87618777

16 Akke, M. and Chazin, W. J. (2001) An open and shut case. Nature Struct. Biol. 8, 910-912

17 Szebenyi, D. M., Obendorf, S. K. and Moffat, K. (1981) Structure of vitamin Ddependent calcium-binding protein from bovine intestine. Nature. 294, 327-332

18 Linse, S., Brodin, P., Drakenberg, T., Thulin, E., Sellers, P., Elmden, K., Grundstrom, T. and Forsén, S. (1987) Structure-function relationships in EF-hand $\mathrm{Ca}^{2+}$-binding proteins. Protein engineering and biophysical studies of calbindin $\mathrm{D}_{9 \mathrm{k}}$. Biochemistry. 26, 6723-6735 
19 Skelton, N. J., Kordel, J. and W.J., C. (1995) Determination of the solution structure of apo calbindin $\mathrm{D}_{9 \mathrm{k}}$ by NMR spectroscopy. J. Mol. Biol. 249, 441-462

20 Kordel, J., Skelton, N. J., Akke, M. and Chazin, W. J. (1993) High resolution solution structure of calcium-loaded calbindin $\mathrm{D}_{9 \mathrm{k}}$. J. Mol. Biol. 231, 711-734

21 Babu, Y. S., Sack, J. S., Greenhough, T. J., Bugg, C. E., Means, A. R. and Cook, W. J. (1985) Three-dimensional structure of calmodulin. Nature. 315, 37-40

22 Kretsinger, R. H., Rudnick, S. E. and Weissman, L. J. (1986) Crystal structure of calmodulin. J. Inorg. Biochem. 28, 289-302

23 Kuboniwa, H., Tjandra, N., Grzesiek, S., Ren, H., Klee, C. B. and Bax, A. (1995) Solution structure of calcium-free calmodulin. Nature Struct. Biol. 2, 768-776

24 Zhang, M., Tanaka, T. and Ikura, M. (1995) Calcium-induced conformational transition revealed by the solution structure of apo calmodulin. Nature Struct. Biol. 2, 758-767

25 Linse, S., Helmersson, A. and Forsen, S. (1991) Calcium binding to calmodulin and its globular domains. J Biol Chem. 266, 8050-8054

26 Barbato, G., Ikura, M., Kay, L. E., Pastor, R. W. and Bax, A. (1992) Backbone dynamics of calmodulin studied by $15 \mathrm{~N}$ relaxation using inverse detected twodimensional NMR spectroscopy: the central helix is flexible. Biochemistry. 31, 5269-5278

27 Chou, J. J., Li, S., Klee, C. B. and Bax, A. (2001) Solution structure of $\mathrm{Ca}^{2+}$ calmodulin reveals flexible hand-like properties of its domains. Nature Struct. Biol. 8, 990-997

28 Heidorn, D. B. and Trewhella, J. (1988) Comparison of the crystal and solution structures of calmodulin and troponin C. Biochemistry. 27, 909-915

29 Ikura, M., Clore, G. M., Gronenborn, A. M., Zhu, G., Klee, C. B. and Bax, A. (1992) Solution structure of a calmodulin-target peptide complex by multidimensional NMR. Science. 256, 632-638

30 Cheung, W. Y. (1970) Cyclic 3',5'-nucleotide phosphodiesterase. Demonstration of an activator. Biochem Biophys Res Commun. 38, 533-538

31 Teo, T. S. and Wang, J. H. (1973) Mechanism of activation of a cyclic adenosine 3':5'-monophosphate phosphodiesterase from bovine heart by calcium ions. Identification of the protein activator as a $\mathrm{Ca} 2+$ binding protein. J Biol Chem. 248, 5950-5955

32 Yazawa, M. and Yagi, K. (1977) Calcium-binding subunit of myosin light chain kinase. J Biochem. 82, 287-289

33 Klee, C. B., Ren, H. and Wang, X. (1998) Regulation of the calmodulin-stimulated protein phosphatase, calcineurin. J Biol Chem. 273, 13367-13370

34 Hook, S. S. and Means, A. R. (2001) $\mathrm{Ca}(2+) / \mathrm{CaM}-d e p e n d e n t ~ k i n a s e s:$ from activation to function. Annu Rev Pharmacol Toxicol. 41, 471-505

35 Gagne, S. M., Tsuda, S., Li, M. X., Smillie, L. B. and Sykes, B. D. (1995) Structures of the troponin $\mathrm{C}$ regulatory domains in the apo and calcium-saturated states. Nat. Struct. Biol. 2, 784-789

36 Strynadka, N. C., Cherney, M., Sielicki, A. R., Li, M. X., Smillie, L. B. and James, M. N. G. (1997) Structural details of a calcium-induced molecular switch: X-ray 
crystallographic analysis of the calcium-saturated N-terminal domain of troponin-C at 1.75 A resolution. J. Mol. Biol. 273, 238-255

37 Leavis, P. C. and Gergely, J. (1984) Thin filament proteins and thin filament-linked regulation of vertebrate muscle contraction. CRC Crit Rev Biochem. 16, 235-305

38 Herzberg, O. and James, M. N. G. (1985) Structure of the calcium regulatory muscle protein troponin $\mathrm{C}$ at 2.8 A resolution. Nature. 313, 653-659

39 Slupsky, C. M. and Sykes, B. D. (1995) NMR solution structure of calcium-saturated skeletal muscle troponin-C. Biochemistry. 34, 15953-15964

40 Li, M. X., Wang, X. and Sykes, B. D. (2004) Structural based insights into the role of troponin in cardiac muscle pathophysiology. J Muscle Res Cell Motil. 25, 559579

41 Donato, R. (2001) S100: a multigenic family of calcium-modulated proteins of the EF-hand type with intracellular and extracellular functional roles. Int. J. Biochem. Cell Biol. 33, 637-668

42 Odink, K., Cerletti, N., Bruggen, J., Clerc, R. G., Tarcsay, L., Zwadlo, G., Gerhards, G., Schlegel, R. and Sorg, C. (1987) Two calcium-binding proteins in infiltrate macrophages of rheumatoid arthritis. Nature. 330, 80-82

43 Van Eldik, L. J. and Griffin, W. S. T. (1994) S100b expression in Alzheimer's disease: Relation to neuropathology in brain regions. Biochim. Biophys. Acta. 1223, 398-403

44 Schafer, B. W. and Heizmann, C. W. (1996) The S100 family of EF-hand calciumbinding proteins: Functions and pathology. Trends Biochem. Sci. 21, 134-140

45 Moore, B. W. (1965) A soluble protein characteristic of the nervous system. Biochem. Biophys. Res. Comm. 19, 739-744

46 Donato, R. (1986) S-100 proteins. Cell Calcium. 7, 123-145

47 Wright, N. T., Prosser, B. L., Varney, K. M., Zimmer, D. B., Schneider, M. F. and Weber, D. J. (2008) S100A1 and calmodulin compete for the same binding site on ryanodine receptor. J Biol Chem. 283, 26676-26683

48 Prosser, B. L., Wright, N. T., Hernandez-Ochoa, E. O., Varney, K. M., Liu, Y., Olojo, R. O., Zimmer, D. B., Weber, D. J. and Schneider, M. F. (2008) S100A1 binds to the calmodulin-binding site of ryanodine receptor and modulates skeletal muscle excitation-contraction coupling. J Biol Chem. 283, 5046-5057

49 Mandinova, A., Atar, D., Schafer, B. W., Spiess, M., Aebi, U. and Heizmann, C. W. (1998) Distinct subcellular localization of calcium binding S100 proteins in human smooth muscle cells and their relocation in response to rises in intracellular calcium. J Cell Sci. 111, 2043-2054

50 Emoto, Y., Kobayashi, R., Akatsuka, H. and Hidaka, H. (1992) Purification and characterization of a new member of the S-100 protein family from human placenta. Biochem. Biophys. Res. Comm. 182, 1246-1253

51 Becker, T., Gerke, V., Kube, E. and Weber, K. (1992) S100P, a novel Ca(2+)binding protein from human placenta. cDNA cloning, recombinant protein expression and $\mathrm{Ca} 2+$ binding properties. Eur J Biochem. 207, 541-547

52 Dempsey, A. C., Walsh, M. P. and Shaw, G. S. (2003) Unmasking the annexin I interaction from the structure of Apo-S100A11. Structure (Camb). 11, 887-897 
53 Drohat, A. C., Amburgey, J. C., Abildgaard, F., Starich, M. R., Baldisseri, D. and Weber, D. J. (1996) Solution structure of rat apo-S100B(beta beta) as determined by NMR spectroscopy. Biochemistry. 35, 11577-11588

54 Drohat, A. C., Tjandra, N., Baldisseri, D. M. and Weber, D. J. (1999) The use of dipolar couplings for determining the solution structure of rat apo-S100B $(\beta \beta)$. Protein Sci. 8, 800-809

55 Kilby, P. M., Van Eldik, L. J. and Roberts, G. C. (1996) The solution structure of the bovine S100B protein dimer in the calcium-free state. Structure. 4, 1041-1052

56 Otterbein, L. R., Kordowska, J., Witte-Hoffmann, C., Wang, C.-L., A. and Dominguez, R. (2002) Crystal structures of S100A6 in the $\mathrm{Ca}^{2+}$-free and $\mathrm{Ca}^{2+}$ bound states: The calcium sensor mechanism of S100 proteins revealed at atomic resolution. Structure. 10, 557-567

57 Rety, S., Sopkova, J., Renouard, M., Osterloh, D., Gerke, V., Tabaries, S., RussoMarie, R. and Lewit-Bentley, A. (1999) The crystal structure of a complex of p11 with the annexin II N-terminal peptide. Nature Struct. Biol. 6, 89-95

58 Rustandi, R. R., Baldisseri, D. M., Inman, K. G., Nizner, P., Hamilton, S. M., Landar, A., Landar, A., Zimmer, D. B. and Weber, D. J. (2002) Three-dimensional solution structure of the calcium-signaling protein apo S100A1 as determined by NMR. Biochemistry. 41, 788-796

59 Vallely, K. M., Rustandi, R. R., Ellis, K. C., Varlamova, O., Bresnick, A. R. and Weber, D. J. (2002) Solution structure of human Mts 1 (S100A4) as determined by NMR spectroscopy. Biochemistry. 41, 12670-12680

60 Lee, Y. C., Volk, D. E., Thiviyanathan, V., Kleerekoper, Q., Gribenko, A. V., Zhang, S., Gorenstein, D. G., Makhatadze, G. I. and Luxon, B. A. (2004) NMR structure of the Apo-S100P protein. J Biomol NMR. 29, 399-402

61 Maler, L., Potts, B. C. and Chazin, W. J. (1999) High resolution solution structure of apo calcyclin and structural variations in the S100 family of calcium-binding proteins. J. Biomol. NMR. 13, 233-247

62 Mittl, P. R. E., Fritz, G., Sargent, D. F., Richmond, T. J., Heizmann, C. W. and Grutter, M. G. (2002) Metal-free MIRAS phasing: structure of apo-S100A3. Acta Cryst. D58, 1255-1261

63 Potts, B. C. M., Smith, J., Akke, M., Macke, T. J., Okazaki, K., Hidaka, H., Case, D. A. and Chazin, W. J. (1995) The structure of calcyclin reveals a novel homodimeric fold for S100 $\mathrm{Ca}^{2+}$-binding proteins. Nature Struct. Biol. 2, 790-796

64 Arnesano, F., Banci, L., Bertini, I., Fantoni, A., Tenori, L. and Viezzoli, M. S. (2005) Structural interplay between calcium(II) and copper(II) binding to S100A13 protein. Angew Chem Int Ed Engl. 44, 6341-6344

65 Brodersen, D. E., Etzerodt, M., Madsen, P., Celis, J. E., Thogersen, H. C., Nyborg, J. and Kjeldgaard, M. (1998) EF-hands at atomic resolution: the structure of human psoriasin (S100A7) solved by MAD phasing. Structure. 6, 477-489

66 Brodersen, D. E., Nyborg, J. and Kjeldgaard, M. (1999) Zinc-binding site of an S100 protein revealed. Two crystal structure of $\mathrm{Ca}^{2+}$-bound human psoriasin (S100A7) in the $\mathrm{Zn}^{2+}$-loaded and $\mathrm{Zn}^{2+}$-free states. Biochemistry. 38, 1695-1704 
67 Drohat, A. C., Baldisseri, D. M., Rustandi, R. R. and Weber, D. J. (1998) Solution structure of calcium-bound rat $\mathrm{S} 100 \mathrm{~B}(\beta \beta)$ as determined by nuclear magnetic resonance spectroscopy. Biochemistry. 37, 2729-2740

68 Ishikawa, K., Nakagawa, A., Tanaka, I., Suzuki, M. and Nishihira, J. (2000) The structure of human MRP8, a member of the S100 calcium-binding protein family, by MAD phasing at 1.9 A resolution. Acta Cryst. D56, 559-566

69 Maler, L., Sastry, M. and Chazin, W. J. (2002) A structural basis for S100 protein specificity derived from comparative analysis of apo and $\mathrm{Ca}^{2+}$-calcyclin. J. Mol. Biol. 317, 279-290

70 Moroz, O. V., Antson, A. A., Murshudov, G. N., Maitland, N. J., Dodson, G. G., Wilson, K. S., Skibshoj, I., Lukanidin, E. M. and Bronstein, I. B. (2001) The threedimensional structure of human S100A12. Acta Cryst. D57, 20-29

71 Sastry, M., Ketchem, R. R., Crescenzi, O., Weber, C., Lubienski, M. J., Hidaka, H. and Chazin, W. J. (1998) The three-dimensional structure of $\mathrm{Ca}^{2+}$-bound calcyclin: Implications for $\mathrm{Ca}^{2+}$-signal transduction by S100 proteins. Structure. 6, 223-231

72 Zhang, H., Wang, G., Ding, Y., Wang, Z., Barraclough, R., Rudland, P. S., Fernig, D. G. and Rao, Z. (2003) The crystal structure at 2A resolution of the Ca2+ -binding protein S100P. J Mol Biol. 325, 785-794

73 Rety, S., Osterloh, D., Arie, J. P., Tabaries, S., Seeman, J., Russo-Marie, F., Gerke, V. and Lewit-Bentley, A. (2000) Structural basis of the $\mathrm{Ca}(2+)$-dependent association between S100C (S100A11) and its target, the N-terminal part of annexin I. Structure Fold Des. 8, 175-184

74 Bhattacharya, S., Large, E., Heizmann, C. W., Hemmings, B. and Chazin, W. J. (2003) Structure of the $\mathrm{Ca} 2+/ \mathrm{S} 100 \mathrm{~B} / \mathrm{NDR}$ kinase peptide complex: insights into S100 target specificity and activation of the kinase. Biochemistry. 42, 14416-14426

75 Inman, K. G., Yang, R., Rustandi, R. R., Miller, K. E., Baldisseri, D. M. and Weber, D. J. (2002) Solution NMR structure of S100B bound to the high-affinity target peptide TRTK-12. J. Mol. Biol. 324, 1003-1014

76 Lee, Y. T., Dimitrova, Y. N., Schneider, G., Ridenour, W. B., Bhattacharya, S., Soss, S. E., Caprioli, R. M., Filipek, A. and Chazin, W. J. (2008) Structure of the S100A6 complex with a fragment from the $\mathrm{C}$-terminal domain of Siah-1 interacting protein: a novel mode for S100 protein target recognition. Biochemistry. 47, 10921-10932

77 McClintock, K. A. and Shaw, G. S. (2003) A novel S100 target conformation is revealed by the solution structure of the Ca2+-S100B-TRTK-12 complex. J Biol Chem. 278, 6251-6257

78 Rustandi, R. R., Baldisseri, D. M. and Weber, D. J. (2000) Structure of the negative regulatory domain of 553 bound to $\operatorname{S} 100 \mathrm{~B}(\beta \beta)$. Nat. Struct. Biol. 7, 570-574

79 Baudier, J. and Cole, R. D. (1988) Interactions between the microtubule-associated t proteins and $\mathrm{S} 100 \mathrm{~b}$ regulate $\mathrm{t}$ protein phosphorylation by the $\mathrm{Ca}^{2+}$-calmodulindependent protein kinase II. J. Biol. Chem. 263, 5876-5883

80 Millward, T. A., Heizmann, C. W., Schafer, B. W. and Hemmings, B. A. (1998) Calcium regulation of $\mathrm{Ndr}$ protein kinase mediated by $\mathrm{S} 100$ calcium- binding proteins. EMBO J. 17, 5913-5922 
81 Stegert, M. R., Tamaskovic, R., Bichsel, S. J., Hergovich, A. and Hemmings, B. A. (2004) Regulation of NDR2 protein kinase by multi-site phosphorylation and the S100B calcium-binding protein. J Biol Chem. 279, 23806-23812

82 Nowotny, M., Spiechowicz, M., Jastrzebska, B., Filipek, A., Kitagawa, K. and Kuznicki, J. (2003) Calcium-regulated interaction of Sgt1 with S100A6 (calcyclin) and other S100 proteins. J Biol Chem. 278, 26923-26928

83 Zimmer, D. B. and Van Eldik, L. J. (1986) Identification of a molecular target for the calcium-modulated protein S100. Fructose-1,6-bisphosphate aldolase. J Biol Chem. 261, 11424-11428

84 Landar, A., Caddell, G., Chessher, J. and Zimmer, D. B. (1996) Identification of an S100A1/S100B target protein: phosphoglucomutase. Cell Calcium. 20, 279-285

85 Endo, H., Takenaga, K., Kanno, T., Satoh, H. and Mori, S. (2002) Methionine aminopeptidase 2 is a new target for the metastasis-associated protein, S100A4. J Biol Chem. 277, 26396-26402

86 Li, C. L., Martinez, V., He, B., Lombet, A. and Perbal, B. (2002) A role for CCN3 (NOV) in calcium signalling. Mol Pathol. 55, 250-261

87 Halachmi, D. and Eilam, Y. (1993) Calcium homeostasis in yeast cells exposed to high concentrations of calcium. Roles of vacuolar H(+)-ATPase and cellular ATP. FEBS Lett. 316, 73-78

88 Mueller, A., Schafer, B. W., Ferrari, S., Weibel, M., Makek, M., Hochli, M. and Heizmann, C. W. (2005) The calcium-binding protein S100A2 interacts with p53 and modulates its transcriptional activity. J Biol Chem. 280, 29186-29193

89 Okada, M., Hatakeyama, T., Itoh, H., Tokuta, N., Tokumitsu, H. and Kobayashi, R. (2004) S100A1 is a novel molecular chaperone and a member of the Hsp70/Hsp90 multichaperone complex. J Biol Chem. 279, 4221-4233

90 Shimamoto, S., Takata, M., Tokuda, M., Oohira, F., Tokumitsu, H. and Kobayashi, R. (2008) Interactions of S100A2 and S100A6 with the tetratricopeptide repeat proteins, Hsp90/Hsp70-organizing protein and kinesin light chain. J Biol Chem. 283, 28246-28258

91 Leclerc, E., Heizmann, C. W. and Vetter, S. W. (2009) RAGE and S100 protein transcription levels are highly variable in human melanoma tumors and cells. Gen Physiol Biophys. 28 Spec No Focus, F65-75

92 Kiryushko, D., Novitskaya, V., Soroka, V., Klingelhofer, J., Lukanidin, E., Berezin, V. and Bock, E. (2006) Molecular mechanisms of $\mathrm{Ca}(2+)$ signaling in neurons induced by the S100A4 protein. Mol Cell Biol. 26, 3625-3638

93 Leclerc, E., Fritz, G., Vetter, S. W. and Heizmann, C. W. (2009) Binding of S100 proteins to RAGE: an update. Biochim Biophys Acta. 1793, 993-1007

94 Streicher, W. W., Lopez, M. M. and Makhatadze, G. I. (2009) Annexin I and annexin II N-terminal peptides binding to S100 protein family members: specificity and thermodynamic characterization. Biochemistry. 48, 2788-2798

95 Fernandez-Fernandez, M. R., Rutherford, T. J. and Fersht, A. R. (2008) Members of the S100 family bind p53 in two distinct ways. Protein Sci. 17, 1663-1670

96 Leclerc, E., Fritz, G., Weibel, M., Heizmann, C. W. and Galichet, A. (2007) S100B and S100A6 differentially modulate cell survival by interacting with distinct RAGE 
(receptor for advanced glycation end products) immunoglobulin domains. J Biol Chem. 282, 31317-31331

97 Emberley, E. D., Niu, Y., Leygue, E., Tomes, L., Gietz, R. D., Murphy, L. C. and Watson, P. H. (2003) Psoriasin interacts with Jab1 and influences breast cancer progression. Cancer Res. 63, 1954-1961

98 Wolf, R., Howard, O. M., Dong, H. F., Voscopoulos, C., Boeshans, K., Winston, J., Divi, R., Gunsior, M., Goldsmith, P., Ahvazi, B., Chavakis, T., Oppenheim, J. J. and Yuspa, S. H. (2008) Chemotactic activity of S100A7 (Psoriasin) is mediated by the receptor for advanced glycation end products and potentiates inflammation with highly homologous but functionally distinct S100A15. J Immunol. 181, 1499-1506

99 Boyd, J. H., Kan, B., Roberts, H., Wang, Y. and Walley, K. R. (2008) S100A8 and S100A9 mediate endotoxin-induced cardiomyocyte dysfunction via the receptor for advanced glycation end products. Circ Res. 102, 1239-1246

100 Vogl, T., Tenbrock, K., Ludwig, S., Leukert, N., Ehrhardt, C., van Zoelen, M. A., Nacken, W., Foell, D., van der Poll, T., Sorg, C. and Roth, J. (2007) Mrp8 and Mrp14 are endogenous activators of Toll-like receptor 4, promoting lethal, endotoxin-induced shock. Nat Med. 13, 1042-1049

101 Rintala-Dempsey, A. C., Santamaria-Kisiel, L., Liao, Y., Lajoie, G. and Shaw, G. S. (2006) Insights into S100 target specificity examined by a new interaction between S100A11 and annexin A2. Biochemistry. 45, 14695-14705

102 Murzik, U., Hemmerich, P., Weidtkamp-Peters, S., Ulbricht, T., Bussen, W., Hentschel, J., von Eggeling, F. and Melle, C. (2008) Rad54B targeting to DNA double-strand break repair sites requires complex formation with S100A11. Mol Biol Cell. 19, 2926-2935

103 Cecil, D. L., Johnson, K., Rediske, J., Lotz, M., Schmidt, A. M. and Terkeltaub, R. (2005) Inflammation-induced chondrocyte hypertrophy is driven by receptor for advanced glycation end products. J Immunol. 175, 8296-8302

104 Sakaguchi, M., Sonegawa, H., Murata, H., Kitazoe, M., Futami, J., Kataoka, K., Yamada, H. and Huh, N. H. (2008) S100A11, an dual mediator for growth regulation of human keratinocytes. Mol Biol Cell. 19, 78-85

105 Wilder, P. T., Lin, J., Bair, C. L., Charpentier, T. H., Yang, D., Liriano, M., Varney, K. M., Lee, A., Oppenheim, A. B., Adhya, S., Carrier, F. and Weber, D. J. (2006) Recognition of the tumor suppressor protein p53 and other protein targets by the calcium-binding protein S100B. Biochim Biophys Acta. 1763, 1284-1297

106 Santamaria-Kisiel, L., Rintala-Dempsey, A. C. and Shaw, G. S. (2006) Calciumdependent and -independent interactions of the S100 protein family. Biochem J. 396, 201-214

107 Sheu, F.-S., Huang, F. L. and Huang, K.-P. (1995) Differential responses of protein kinase $\mathrm{C}$ substrates (MARCKS, neuromodulin and neurogranin) phosphorylation to calmodulin and S100. Arch. Biochem. Biophys. 316, 335-342

108 Polyakov, A. A., Huber, P. A., Marston, S. B. and Gusev, N. B. (1998) Interaction of isoforms of S100 protein with smooth muscle caldesmon. FEBS Lett. 422, 235-239 
109 Sorci, G., Agneletti, A. L., Bianchi, R. and Donato, R. (1998) Association of S100B with intermediate filaments and microtubules in glial cells. Biochim. Biophys. Acta. 1448, 277-289

110 Frizzo, J. K., Tramontina, F., Bortoli, E., Gottfried, C., Leal, R. B., Lengyel, I., Donato, R., Dunkley, P. R. and Goncalves, C. A. (2004) S100B-mediated inhibition of the phosphorylation of GFAP is prevented by TRTK-12. Neurochem Res. 29, 735-740

111 Ferguson, P. L. and Shaw, G. S. (2004) Human S100B protein interacts with the Escherichia coli division protein FtsZ in a calcium-sensitive manner. J Biol Chem. 279, 18806-18813

112 Ivanenkov, V. V., Jamieson Jr., G. A., Gruenstein, E. and Dimlich, R. V. W. (1995) Characterization of S-100b binding epitopes: Identification of a novel target, the actin capping protein CapZ. J. Biol. Chem. 270, 14651-14658

113 Seemann, J., Weber, K. and Gerke, V. (1997) Annexin I targets S100C to early endosomes. FEBS Lett. 413, 185-190

114 Hofmann, M. A., Drury, S., Fu, C., Qu, W., Taguchi, A., Lu, Y., Avila, C., Kambham, N., Bierhaus, A., Nawroth, P., Neurath, M. F., Slattery, T., Beach, D., McClary, J., Nagashima, M., Morser, J., Stern, D. and Schmidt, A. M. (1999) RAGE mediates a novel proinflammatory axis: a central cell surface receptor for S100/calgranulin polypeptides. Cell. 97, 889-901

115 Huttunen, H. J., Kuja-Panula, J., Sorci, G., Agneletti, A. L., Donato, R. and Rauvala, H. (2000) Coregulation of neurite outgrowth and cell survival by amphoterin and S100 proteins through receptor for advanced glycation end products (RAGE) activation. J Biol Chem. 275, 40096-40105

116 Arumugam, T., Simeone, D. M., Schmidt, A. M. and Logsdon, C. D. (2004) S100P stimulates cell proliferation and survival via receptor for activated glycation end products (RAGE). J Biol Chem. 279, 5059-5065

117 Most, P., Remppis, A., Weber, C., Bernotat, J., Ehlermann, P., Pleger, S. T., Kirsch, W., Weber, M., Uttenweiler, D., Smith, G. L., Katus, H. A. and Fink, R. H. (2003) The $\mathrm{C}$ terminus (amino acids 75-94) and the linker region (amino acids 42-54) of the $\mathrm{Ca} 2+-$ binding protein S100A1 differentially enhance sarcoplasmic $\mathrm{Ca} 2+$ release in murine skinned skeletal muscle fibers. J Biol Chem. 278, 26356-26364

118 Most, P., Remppis, A., Pleger, S. T., Loffler, E., Ehlermann, P., Bernotat, J., Kleuss, C., Heierhorst, J., Ruiz, P., Witt, H., Karczewski, P., Mao, L., Rockman, H. A., Duncan, S. J., Katus, H. A. and Koch, W. J. (2003) Transgenic overexpression of the Ca2+-binding protein S100A1 in the heart leads to increased in vivo myocardial contractile performance. J Biol Chem. 278, 33809-33817

119 Arcuri, C., Giambanco, I., Bianchi, R. and Donato, R. (2002) Annexin V, annexin VI, S100A1 and S100B in developing and adult avian skeletal muscles. Neuroscience. 109, 371-388

120 Garbuglia, M., Verzini, M. and Donato, R. (1998) Annexin VI binds S100A1 and S100B and blocks the ability of S100A1 and S100B to inhibit desmin and GFAP assemblies into intermediate filaments. Cell Calcium. 24, 177-191 
121 Zeng, F. Y., Gerke, V. and Gabius, H. J. (1993) Identification of annexin II, annexin VI and glyceraldehyde-3-phosphate dehydrogenase as calcyclin-binding proteins in bovine heart. Int J Biochem. 25, 1019-1027

122 Fernandez-Fernandez, M. R., Veprintsev, D. B. and Fersht, A. R. (2005) Proteins of the S100 family regulate the oligomerization of p53 tumor suppressor. Proc Natl Acad Sci U S A. 102, 4735-4740

123 Grigorian, M., Andressen, S., Tulchinsky, E., Kriajevska, M., Carlberg, C., Kruse, C., Cohn, M., Ambartsumian, N., Christensen, A., Selivanova, G. and Lukanidin, E. (2001) Tumor suppressor p53 protein is a new target for the metastasis-associated mts1/S100A4 protein. J Biol Chem. 276, 22699-22708

124 Chen, H., Fernig, D. G., Rudland, P. S., Sparks, A., Wilkinson, M. C. and Barraclough, R. (2001) Binding to intracellular targets of the metastasis-inducing protein, S100A4 (p9Ka). Biochem Biophys Res Commun. 286, 1212-1217

125 Baudier, J., Delphin, C., Grunwald, D., Khochbin, S. and Lawrence, J. J. (1992) Characterization of the tumor suppressor protein p53 as a protein knase $\mathrm{C}$ substrate and a S100b-binding protein. Proc. Natl. Acad. Sci. USA. 89, 11627-11631

126 Filipek, A., Jastrzebska, B., Nowotny, M. and Kuznicki, J. (2002) CacyBP/SIP, a calcyclin and Siah-1-interacting protein, binds EF-hand proteins of the S100 family. J Biol Chem. 277, 28848-28852

127 Zhang, S., Wang, G., Fernig, D. G., Rudland, P. S., Webb, S. E., Barraclough, R. and Martin-Fernandez, M. (2005) Interaction of metastasis-inducing S100A4 protein in vivo by fluorescence lifetime imaging microscopy. Eur Biophys J. 34, 19-27

128 Pearlstone, J. R., Sykes, B. D. and Smillie, L. B. (1997) Interactions of structural C and regulatory $\mathrm{N}$ domains of troponin $\mathrm{C}$ with repeated sequence motifs in troponin $\mathrm{I}$. Biochemistry. 36, 7601-7606

129 McKay, R. T., Tripet, B. P., Hodges, R. S. and Sykes, B. D. (1997) Interaction of the second binding region of troponin I with the regulatory domain of skeletal muscle troponin C as determined by NMR spectroscopy. J. Biol. Chem. 272, 28494-28500

130 Landar, A., Rustandi, R. R., Weber, D. J. and Zimmer, D. B. (1998) S100A1 utilizes different mechanisms for interacting with calcium-dependent and calciumindependent target proteins. Biochemistry. 37, 17429-17438

131 Barber, K. R., McClintock, K. A., Jamieson, G. A., Jr., Dimlich, R. V. and Shaw, G. S. (1999) Specificity and $\mathrm{Zn}^{2+}$ enhancement of the S100B binding epitope TRTK-12. J. Biol. Chem. 274, 1502-1508

132 Li, Z. H., Spektor, A., Varlamova, O. and Bresnick, A. R. (2003) Mts1 regulates the assembly of nonmuscle myosin-IIA. Biochemistry. 42, 14258-14266

133 Takenaga, K., Nakamura, Y., Sakiyama, S., Hasegawa, Y., Sato, K. and Endo, H. (1994) Binding of pEL98 protein, an S100-related calcium-binding protein, to nonmuscle tropomyosin. J Cell Biol. 124, 757-768

134 Delphin, C., Ronjat, M., Deloulme, J. C., Garin, G., Debussche, L., Higashimoto, Y., Sakaguchi, K. and Baudier, J. (1999) Calcium-dependent interaction of S100B with the C-terminal domain of the tumor suppressor p53. J. Biol. Chem. 274, 1053910544 
135 Garbuglia, M., Verzini, M., Sorci, G., Bianchi, R., Giambanco, I., Agneletti, A. L. and Donato, R. (1999) The calcium-modulated proteins, S100A1 and S100B, as potential regulators of the dynamics of type III intermediate filaments [In Process Citation]. Braz. J. Med. Biol. Res. 32, 1177-1185

136 Filipek, A., Wojda, U. and Lesniak, W. (1995) Interaction of calcyclin and its cyanogen bromide fragments with annexin II and glyceraldehyde 3-phosphate dehydrogenase. Int J Biochem Cell Biol. 27, 1123-1131

137 Zimmer, D. B. and Dubuisson, J. G. (1993) Identification of an S100 target protein: glycogen phosphorylase. Cell Calcium. 14, 323-332

138 Ruse, M., Lambert, A., Robinson, N. A., Ryan, D., Shon, K. and Eckert, R. L. (2001) S100A7, S100A10, and S100A11 are transglutaminase substrates. Biochemistry. 40, 3167-3173

139 Donier, E., Rugiero, F., Okuse, K. and Wood, J. N. (2005) Annexin II light chain p11 promotes functional expression of acid-sensing ion channel ASIC1a. J Biol Chem. 280, 38666-38672

140 Zhang, S., Wang, G., Liu, D., Bao, Z., Fernig, D. G., Rudland, P. S. and Barraclough, R. (2005) The C-terminal region of S100A4 is important for its metastasis-inducing properties. Oncogene. 24, 4401-4411

141 Kriajevska, M. V., Cardenas, M. N., Grigorian, M. S., Ambartsumian, N. S., Georgiev, G. P. and Lukanidin, E. M. (1994) Non-muscle myosin heavy chain as a possible target for protein encoded by metastasis-related mts-1 gene. J Biol Chem. 269, 19679-19682

142 Ford, H. L., Silver, D. L., Kachar, B., Sellers, J. R. and Zain, S. B. (1997) Effect of Mts1 on the structure and activity of nonmuscle myosin II. Biochemistry. 36, 1632116327

143 Ford, H. L. and Zain, S. B. (1995) Interaction of metastasis associated Mts1 protein with nonmuscle myosin. Oncogene. 10, 1597-1605

144 Kim, E. J. and Helfman, D. M. (2003) Characterization of the metastasis-associated protein, S100A4. Roles of calcium binding and dimerization in cellular localization and interaction with myosin. J Biol Chem. 278, 30063-30073

145 Gribenko, A. V., Guzman-Casado, M., Lopez, M. M. and Makhatadze, G. I. (2002) Conformational and thermodynamic properties of peptide binding to the human S100P protein. Protein Sci. 11, 1367-1375

146 Drohat, A. C., Nenortas, E., Beckett, D. and Weber, D. J. (1997) Oligomerization state of S100B at nanomolar concentration determined by large-zone analytical gel filtration chromatography. Protein Sci. 6, 1577-1582

147 Fano, G., Biocca, S., Fulle, S., Mariggio, M., Belia, S. and Calissano, P. (1995) The S100: A protein family in search of a function. Prog. Neurobiol. 46, 71-82

148 Odink, K., Cerletti, N., Bruggen, J., Clerc, R. G., Tarcsay, L., Zwadlo, G., Gerhards, G., Schlegel, R. and Sorg, C. (1987) Two calcium-binding proteins in infiltrate macrophages of rheumatoid arthritis. Nature. 330, 80-82

149 Itou, H., Yao, M., Fujita, I., Watanabe, N., Suzuki, M., Nishihira, J. and Tanaka, I. (2002) The crystal structure of human MRP14 (S100A9), a $\mathrm{Ca}(2+)$-dependent regulator protein in inflammatory process. J Mol Biol. 316, 265-276 
150 Propper, C., Huang, X., Roth, J., Sorg, C. and Nacken, W. (1999) Analysis of the MRP8-MRP14 protein-protein interaction by the two-hybrid system suggests a prominent role of the C-terminal domain of S100 proteins in dimer formation. J Biol Chem. 274, 183-188

151 Wang, G., Rudland, P. S., White, M. R. and Barraclough, R. (2000) Interaction in vivo and in vitro of the metastasis-inducing S100 protein, S100A4 (p9Ka) with S100A1. J. Biol. Chem. 275, 11141-11146

152 Yang, Q., O'Hanlon, D., Heizmann, C. W. and Marks, A. (1999) Demonstration of heterodimer formation between S100B and S100A6 in the yeast two-hybrid system and human melanoma. Exp. Cell Res. 246, 501-509

153 Shaw, G. S., Hodges, R. S., Kay, C. M. and Sykes, B. D. (1994) Relative stabilities of synthetic peptide homo- and heterodimeric troponin-C domains. Protein Sci. 3, 1010-1019

154 Lehrer, S. S. and Y., Q. (1990) Unfolding/refolding studies of smooth muscle tropomyosin. Evidence for a chain exchange mechanism in the preferential assembly of the native heterodimer. J. Biol. Chem. 265, 1134 -1138

155 Tarabykina, S., Scott, D. J., Herzyk, P., Hill, T. J., Tame, J. R., Kriajevska, M., Lafitte, D., Derrick, P. J., Dodson, G. G., Maitland, N. J., Lukanidin, E. M. and Bronstein, I. B. (2001) The dimerization interface of the metastasis-associated protein S100A4 (Mts1): in vivo and in vitro studies. J Biol Chem. 276, 24212-24222

156 Wang, G., Zhang, S., Fernig, D. G., Martin-Fernandez, M., Rudland, P. S. and Barraclough, R. (2005) Mutually antagonistic actions of S100A4 and S100A1 on normal and metastatic phenotypes. Oncogene. 24, 1445-1454

157 Deloulme, J. C., Assard, N., Mbele, G. O., Mangin, C., Kuwano, R. and Baudier, J. (2000) S100A6 and S100A11 are specific targets of the calcium- and zinc-binding S100B protein in vivo. J. Biol. Chem. 275, 35302-35310

158 Tarabykina, S., Kriajevska, M., Scott, D. J., Hill, T. J., Lafitte, D., Derrick, P. J., Dodson, G. G., Lukanidin, E. and Bronstein, I. (2000) Heterocomplex formation between metastasis-related protein S100A4 (Mts1) and S100A1 as revealed by the yeast two-hybrid system. FEBS Lett. 475, 187-191

159 Wang, G., Zhang, S., Fernig, D. G., Spiller, D., Martin-Fernandez, M., Zhang, H., Ding, Y., Rao, Z., Rudland, P. S. and Barraclough, R. (2004) Heterodimeric interaction and interfaces of S100A1 and S100P. Biochem J. 382, 375-383

160 Johnsson, N., Marriott, G. and Weber, K. (1988) p36, the major cytoplasmic substrate of src tyrosine protein kinase, binds to its $\mathrm{p} 11$ regulatory subunit via a short amino-terminal amphiphatic helix. EMBO J. 7, 2435-2442

161 Svenningsson, P., Chergui, K., Rachleff, I., Flajolet, M., Zhang, X., El Yacoubi, M., Vaugeois, J. M., Nomikos, G. G. and Greengard, P. (2006) Alterations in 5-HT1B receptor function by $\mathrm{p} 11$ in depression-like states. Science. 311, 77-80

162 Beaton, A. R., Rodriguez, J., Reddy, Y. K. and Roy, P. (2002) The membrane trafficking protein calpactin forms a complex with bluetongue virus protein NS3 and mediates virus release. Proc Natl Acad Sci U S A. 99, 13154-13159

163 Choi, J., Chang, J. S., Song, M. S., Ahn, B. Y., Park, Y., Lim, D. S. and Han, Y. S. (2003) Association of hepatitis B virus polymerase with promyelocytic leukemia 
nuclear bodies mediated by the S100 family protein p11. Biochem Biophys Res Commun. 305, 1049-1056

164 Poon, W. Y., Malik-Hall, M., Wood, J. N. and Okuse, K. (2004) Identification of binding domains in the sodium channel $\mathrm{Na}(\mathrm{V}) 1.8$ intracellular $\mathrm{N}$-terminal region and annexin II light chain p11. FEBS Lett. 558, 114-118

165 Rescher, U. and Gerke, V. (2008) S100A10/p11: family, friends and functions. Pflugers Arch. 455, 575-582

166 Todoroki, H., Kobayashi, R., Watanabe, M., Minami, H. and Hidaka, H. (1991) Purification, characterization and partial sequence analysis of a newly identified EFhand type $13-\mathrm{kDa} \mathrm{Ca}^{2+}$-binding protein from smooth muscle and non-muscle tissues. J. Biol. Chem. 266, 18668-18673

167 Mailliard, W. S., Haigler, H. T. and Schlaepfer, D. D. (1996) Calcium-dependent binding of S100C to the N-terminal domain of annexin I. J. Biol. Chem. 271, 719725

168 Chang, N., Sutherland, C., Hesse, E., Winkfein, R., Wiehler, W. B., Pho, M., Veillette, C., Li, S., Wilson, D. P., Kiss, E. and Walsh, M. P. (2007) Identification of a novel interaction between the $\mathrm{Ca}(2+)$-binding protein S100A11 and the $\mathrm{Ca}(2+)$ and phospholipid-binding protein annexin A6. Am J Physiol Cell Physiol. 292, C1417-1430

169 Lecona, E., Turnay, J., Olmo, N., Guzman-Aranguez, A., Morgan, R. O., Fernandez, M. P. and Lizarbe, M. A. (2003) Structural and functional characterization of recombinant mouse annexin A11: influence of calcium binding. Biochem J. 373, 437-449

170 Naka, M., Qing, Z. X., Sasaki, T., Kise, H., Tawara, I., Hamaguchi, S. and Tanaka, T. (1994) Purification and characterization of a novel calcium-binding protein, S100C, from porcine heart. Biochim. Biophys. Acta. 1223, 348-353

171 Sakaguchi, M., Miyazaki, M., Sonegawa, H., Kashiwagi, M., Ohba, M., Kuroki, T., Namba, M. and Huh, N. H. (2004) PKCalpha mediates TGFbeta-induced growth inhibition of human keratinocytes via phosphorylation of S100C/A11. J Cell Biol. 164, 979-984

172 Deloulme, J. C., Gentil, B. J. and Baudier, J. (2003) Monitoring of S100 homodimerization and heterodimeric interactions by the yeast two-hybrid system. Microsc Res Tech. 60, 560-568 


\section{Chapter 2}

\section{COMPARATIVE ANALYSIS OF S100A11 AND S100A10 PROTEINS ${ }^{1}$}

\subsection{Introduction}

$\mathrm{S} 100 \mathrm{~A} 10$ is a unique member of the S100 family, unable to bind calcium due to substitutions and deletions of calcium-coordinating residues in both EF-hands. Despite this deficiency, calcium-free S100A10 [1] has a similar "open" conformation of other S100 proteins in their calcium-bound forms, particularly $\mathrm{Ca}^{2+}-\mathrm{S} 100 \mathrm{~A} 11$ [2] (Figure 1.11). Thus, S100A10 forms a tight complex with annexin A2 and a variety of other proteins in a calcium independent manner $[3,4]$. To date, S100A10 is the only protein to display this property.

More than 25 years have passed since S100A10 was first identified in complex with the phospholipid-binding protein annexin A2 [5-7] and 10 years since its molecular structure was reported [1], revealing the ability of S100A10 to form a strong complex with annexin A2 in vivo in a calcium-independent manner. However, there is a lack of understanding of how the primary sequence of S100A10 provides it with a unique permanently open conformation. Previous mutational and structural studies have shown that subtle differences in sequence amongst the EF-hand motifs confer them structural and functional specificity [8-10]. Given the high sequence similarity between S100A10 and S100A11 (41\%) the aim of these studies is o identify regions where significant

\footnotetext{
${ }^{1}$ Parts of this chapter have been taken from a published manuscript. Santamaria-Kisiel, L. and Shaw, G.S. 2011. Identification of Regions Responsible for the Open Conformation of S100A10 using Chimaeric S100A11-S100A10 Proteins. Biochem J. 434, 37-48
} 
disparities occur between the two proteins and evaluate the contributions of these amino acids to the stabilization of the open form in S100A10.

This chapter presents a series of detailed analyses of S100A11 and S100A10 in an effort to identify differences in sequence and structure between these two proteins that may contribute to the open conformation of S100A10. The strategy used in these studies parallels a highly complementary approach reported by Chazin and coworkers, with the goal of re-engineering calbindin $\mathrm{D}_{9 \mathrm{~K}}$ to undergo a calcium-induced conformational change similar to that of calmodulin [10]

The analysis described here used a combination of sequence alignment comparisons; $\alpha$-carbon distance measurements; inter-residue contact analyses and hydrophobic surface area assessments using the three-dimensional structures of S100A11 and S100A10. The comparison revealed some disparities between apo-S100A11 and S100A10 at calcium coordinating positions and the regions involving helix III. In addition, an increased number of hydrogen bonds within the calcium binding loops and through out the protein were identified in S100A10, compared to apo-S100A11, which could aid to stabilize the active structure of S100A10.

\subsection{Materials and Methods}

\subsubsection{Sequence alignments}

The sequence of human S100A10 (GenBank: CAG46959.1) was aligned with sequences of dimeric S100 proteins for which three-dimensional structures have been

reported in the calcium-free state (e.g. S100A1 (PDB: 1K2H GI:5620558); S100A2 (PDB: 1RGI), S100A3 (PDB: 1KSO), S100A4 (PDB: 1M3), S100A5 (PDB: 1KAX), 
S100A6 (PDB: 1K9P), S100A11 (PDB: 1NSH), S100A12 (PDB: 2WCE), S100A13 (PDB 1YUS), S100B (PDB: 1B4C) and S100P (PDB: 1OZO)). In addition, multiple sequence alignments were generated for various species of S100A11 and S100A10 proteins to determine amino acid conservation. The alignments of all sequences were constructed using CLUSTALW2 [11] through the web interface provided by The European Bioinformatics Institute (http://www.ebi.ac.uk/Tools/clustalw2/) [12], using default settings (Gonnet250 weight matrix, gap opening penalty of 10, gap extension penalty of 0.2 , Protein Gap Open Penalty $=10.0$ ).

\subsubsection{Molecular graphics and structure visualization}

The X-ray crystallographic structure of human S100A10 in complex with annexin A2 peptide (PDB: 1BT6) [1] was compared to the solution NMR structure of apoS100A11 (PDB: 1NSH) [13] and the X-ray structure of $\mathrm{Ca}^{2+}-\mathrm{S} 100 \mathrm{~A} 11$ in complex with the N-terminus of annexin A1 (PDB: 1QLS) [2] using the molecular graphic interfaces SwissPDB Viewer [14] and MacPymol (http://delsci.com/macpymol/). Further, pair wise comparisons and root mean squared deviations (rmsd) were completed for all proteins using the program SwissPdb Viewer to identify regions in which S100A10 and S100A11 differ. To visualize the projection of amino acids in alpha helices of S100A11 and S100A10 helical wheel representations were plotted utilizing the web-based helical wheel applet at http://cti.itc.virginia.edu/ cmg/Demo/ wheel/wheelApp.html (University of Virginia). 


\subsubsection{Interhelical angle measurements}

Interhelical angles were calculated using INTERHLX (obtained from nmr.uhnres. utoronto.ca/ikura/datasoft.html. University of Toronto). This program represents each helix by a straight-line vector with the coordinates of the vector endpoints defined as the average of the first (or last) ten atom coordinate positions of each helix. The angle is the dot product of the two helical regions with its sign defined as described previously [15].

\subsubsection{Side chain accessible surface area}

The fractional accessible surface areas for side chain residues in apo-S100A11, $\mathrm{Ca}^{2+}-\mathrm{S} 100 \mathrm{~A} 11$ and S100A10 (1BT6) were calculated from their three-dimensional structures using the web-based software VADAR (Volume Area Dihedreal Angle Calculator) (http://redpoll.pharmacy.ualberta.ca/vadar/index.html) [16]. The accessible

surface areas for $\mathrm{Ca}^{2+} \mathrm{S} 100 \mathrm{~A} 11$ and $\mathrm{S} 100 \mathrm{~A} 10$ were calculated after removing the annexin peptides coordinates from the structures.

\subsubsection{Carbon alpha distances}

The distance (D) between each pair of $\alpha$-carbon atoms $(i, j)$ in apo-S100A11, $\mathrm{Ca}^{2+}-\mathrm{S} 100 \mathrm{~A} 11$ and S100A10 was measured from the structure coordinates using the following equation:

$$
\left.D i, j=\sqrt{ }\left(\left(i_{x}-j_{x}\right)^{2}+i_{y}-j_{y}\right)^{2}+\left(i_{z}-j_{z}\right)^{2}\right)
$$

Distance differences (DD) for the same pair of atoms in any of the two structures to be compared were calculated using the formula:

$$
\mathrm{DD}=\mathrm{Di}, \mathrm{j} \text { Apo-S100A11-Di,j S100A10 }
$$


To simplify the comparisons only distances between $\alpha$-carbon atoms smaller than $8 \AA$ were considered. This cut off was derived from the distance between $\alpha$-carbon atoms of the majority of residues in the N-terminus of helix III and the C-terminus of helix IV in apo-S100A11, which increases considerably upon reorientation of helix III in $\mathrm{Ca}^{2+}-\mathrm{S} 100 \mathrm{~A} 11$ and S100A10 (i.e $\mathrm{D}_{\mathrm{V} 55, \mathrm{G} 82}=7.98 \AA$ in apo-S100A11 and $\mathrm{D}_{\mathrm{V} 55, \mathrm{G} 82}=15.50$ $\AA$ in $\left.\mathrm{Ca}^{2+}-\mathrm{S} 100 \mathrm{~A} 11\right)$. Alpha carbon distances calculated were displayed in each threedimensional structure using MacPymol (http://delsci.com/macpymol) to illustrate differences in backbone folding amongst the structures.

\subsubsection{Inter-residue contact analysis}

Amino acid interactions were determined based on the distance between the sidechain of each residue in the structures (apo-S100A11, $\mathrm{Ca}^{2+}-\mathrm{S} 100 \mathrm{~A} 11$ and S100A10). Two residues were defined to be in contact if the distance between the side chain atoms was within $6 \AA$. Inter-residue distances were evaluated using SwissPDB Viewer in addition to Contact Map Analysis calculated using the web interface CMA at http://ligin.weizmann.ac.il/cma/ [17].

\subsubsection{Helical limits}

Sequence alignments of rabbit-S100A11 and human-S100A10 were used to define helical regions, so that the helices were the same length in all structures. Helix boundaries were defined flanking the calcium-binding loops such that the incoming helix ended at the hydrophobic residue preceding the first residue of the loop. In apo-S100A11 this corresponds to Y20 and L63. The exiting helix started at the residue immediately after the bidentate glutamate that ends the calcium-binding loop. For instance, F35 was 
chosen to be the first residue of helix II in apo-S100A11, even though the last three residues from the calcium-binding loop (32-35) constitute the first turn of this helix. These limits were used for all comparative studies and to define the chimaeric proteins designed in Chapter 3. In contrast, to obtain all interhelical angles the secondary structures were defined based on the $\varphi, \psi$ angles from the Ramachandran plot of each individual structure.

\subsection{Results and Discussion}

It is not clear how S100A10 is able to adopt an open structure in the absence of calcium binding, resembling that of $\mathrm{Ca}^{2+}-\mathrm{S} 100 \mathrm{~A} 11$. As with most $\mathrm{S} 100$ proteins, S100A10 shares significant sequence identity with S100A11 (41\%). However, the open conformation of S100A10 indicates there must be key differences in its amino acid composition compared to S100A11, which likely contribute to its altered conformation. To identify the sequences and regions that could contribute and stabilize the open conformation of S100A10, comprehensive comparative analyses of S100A11 and S100A10 were conducted. The studies used a combination of: (1) sequence alignments comparisons, to identify important amino acids substitutions; (2) three-dimensional structure examination; (3) $\alpha$-carbon distances measurements, to determine differences in backbone folding; (4) inter-residue contact analyses and (5) hydrophobic surface areas calculations, to evaluate the balance of solvated polar and hydrophobic residues [8].

\subsubsection{Differences in the amino acid composition of S100A10}

Sequence variations within the S100 protein family were used as a starting point in an effort to determine differences at the amino acid level that could have an important 
role in stabilizing the open form of S100A10. Generally, if a residue is highly conserved in all S100 proteins it would be less likely to contribute to the structural differences in S100A10. Similarly, if there is widespread variation at a particular site, it is equally unlikely to be important for the stabilization of the active form of S100A10.

Figure 2.1 shows the multiple sequence alignment of S100A10 and other S100 proteins for which the "closed" three-dimensional structures in the absence of calcium have been reported. The sequence of S100A10 shows differences primarily in the composition of the calcium-binding loops. The pseudo canonical EF-hand has a threeresidue deletion and lacks the conserved glutamate at position $-\mathrm{Z}$ of the calcium coordination sphere. In addition, S100A10 is the only protein that contains a penultimate negatively charged residue at the end of loop I (E28) that corresponds to a polar, neutral $(\mathrm{T} / \mathrm{S})$ or basic $(\mathrm{K})$ residue in the remaining S100 proteins, possibly affecting the helix dipole of helix II. In the second calcium-binding site S100A10 contains two critical substitutions (C61 and S70) at calcium coordinating positions y and $-\mathrm{z}$. Replacements occur at the conserved aspartate at the N-terminus of the loop $\left(\mathrm{Ca}^{2+}\right.$ coordinating position 3) and at the bidentate glutamate found at the end of the loop (position 12). The residue at position 12 in either of the EF-hand loops provides two oxygens for calcium coordination and its replacement leads to the inability to bind calcium, as has been shown by site directed mutagenesis in S100B [18], calmodulin [19, 20] and calbindin $\mathrm{D}_{9 \mathrm{~K}}[21,22]$.

Further, all EF-hand calcium-binding loops are rich in negatively charged residues $(\sim 4 /$ site $)$, which provide carboxylate ligands for ion coordination. Remarkably, site II in S100A10 contains only two acidic residues (D59, D63) and more neutral (Q60, 


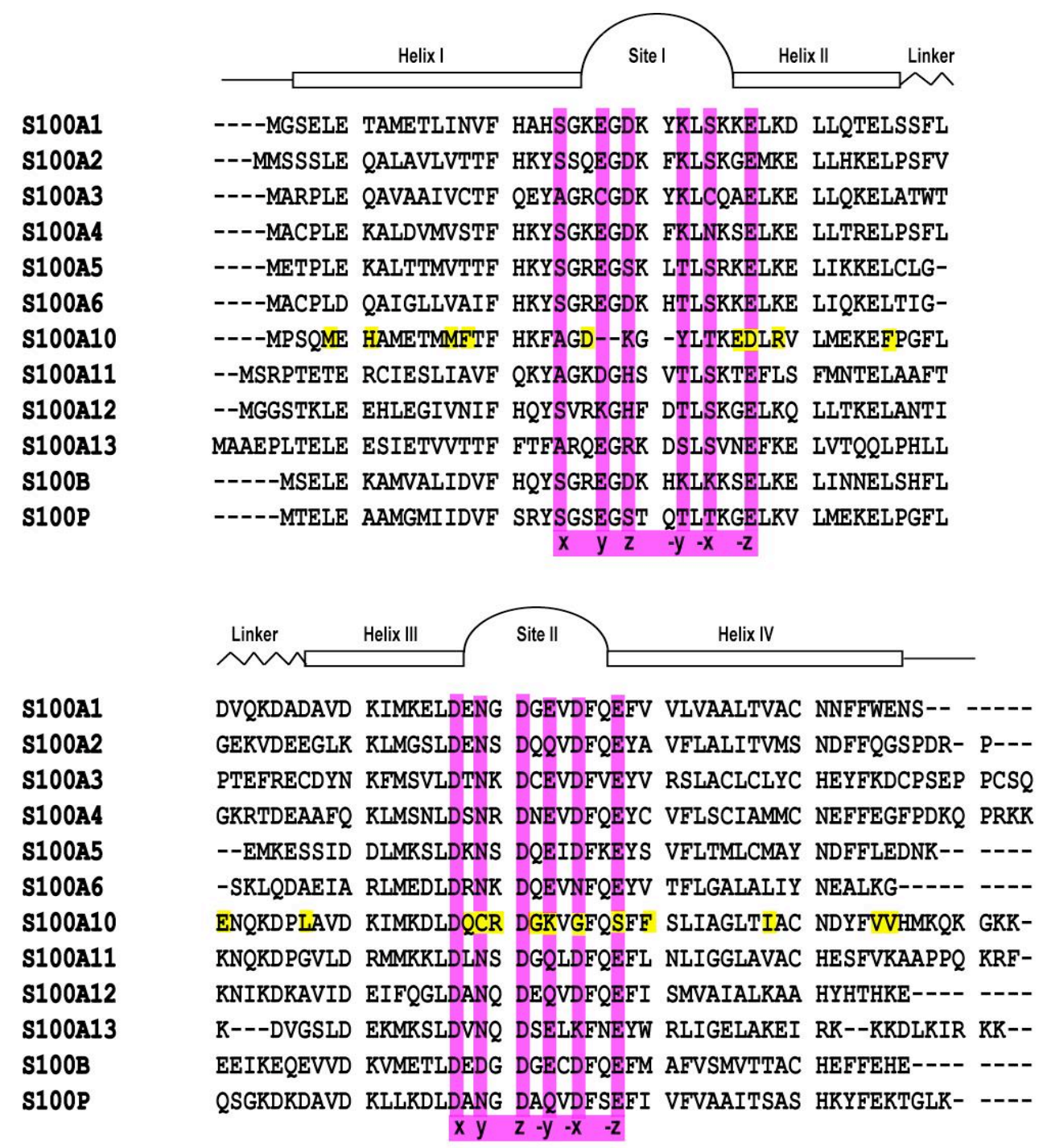

Figure 2.1 Multiple sequence alignment of S100 proteins .

Multiple sequence alignment of S100 proteins for which three-dimensional structures in the apo-form have been reported performed using Clustal W 2.01. The $\mathrm{Ca}^{2+}$-coordinating positions are highlighted in pink. Residues unique in the S100A10 sequence are highlighted in yellow and the secondary structures shown above the sequences. Major differences between the primary sequence of S100A10 and other S100 proteins occur in the calcium-binding sites and the $\mathrm{N}$-terminal portions of the helices and the linker region. 
R62 and K65) amino acids, which contribute to its net charge of 0 at a $\mathrm{pH}$ near 7.0. In other S100 proteins this canonical loop is more negatively charged and must overcome the destabilizing electrostatic interactions in their loop arrangements [23].

It is worth noting that distinctive substitutions in S100A10 do not occur randomly along the protein sequence but seem to be grouped at the $\mathrm{N}$-terminal portion of each $\alpha$ helix and the linker region, while higher identity or conservative substitutions occur near the C-terminus of each section (Figure 2.1). For example, residues at the N-terminus of the linker in $\mathrm{S} 100 \mathrm{~A} 10\left({ }^{38} \mathrm{FPGF}^{41}\right)$ are nearly identical to those in S100P (LPGF) but significantly different from those of S100A1 (LSSF), S100B (LSHF), S100A12 (LANT), S100A6 (LITG) and S100A11 (LAAF), whereas the C-terminus is identical to S100A11 (NQKD) and highly similar to S100A1 (VQKD), S100A12 (NIKD) and S100P (SGKD).

Three S100 proteins share high sequence identity and similarity with S100A10: S100A1 (48\%, 72\%), S100P (45\%, 67\%) and S100A11 (41\%, 63\%) and could be used as templates to test hypotheses as to which residues govern the permanently open form of S100A10. S100A11 is a particularly interesting candidate as its $\mathrm{Ca}^{2+}$-bound form presents a nearly identical fold to that of S100A10 (discussed later in this chapter). In addition, $\mathrm{Ca}^{2+}-\mathrm{S} 100 \mathrm{~A} 11$ can form complexes with proteins of the annexin family and potentially intervene in membrane trafficking events similarly to S100A10 [24, 25]. This resemblance in structure and biological function has not been observed for any other pair of S100 proteins. 


\subsubsection{Sequence similarity between S100A10 and S100A11}

S100A10 and S100A11 are highly related proteins (identity $41 \%$, similarity $63 \%$, conservation 93\%), well conserved amongst species. They display strong sequence identity in the linker region, helix III and the N-terminus of helix IV (Figure 2.1 and 1.13). Interestingly, these are the regions that form the surface for biological target recognition, and could explain the capability of these two proteins to interact with similar biological partners, such as annexin A2.

The most evident primary sequence differences between S100A10 and S100A11, as with other S100 proteins, are a three-residue gap in the non-canonical EF-hand and substitutions in calcium coordinating residues in both EF-hand loops. Further, amino acid substitutions appear to be clustered at the N-terminus of the helices. A helical wheel representation (Figure 2.2) was plotted to appreciate the distribution of the amino acid substitutions in S100A10 compared to S100A11. The figure shows that all the helices in the proteins are amphipathic, with hydrophobic residues facing their inner portions. Moreover, the representation illustrates how differences in amino acid composition between S100A10 and S100A11 are enriched on the accessible faces of the helices, except for helix I where substitutions occur all throughout the helix. This suggests that the buried core of S100A10 is more similar to that in S100A11 than the exposed outer portions of the helices.

Overall, sequence alignment comparisons show that S100A10 presents unique amino acid composition in the calcium binding loops and the N-terminus of helices II, III, IV and the linker region, which correspond to the exposed face of the helices. There are 
54 non-identical residues comparing human-S100A10 and rabbit-S100A11 that could potentially lead to differences in residues interactions allowing S100A10 to adopt a calcium-bound like conformation in the absence of calcium. Moreover, S100A10 contains 22 unique residues in its sequence, with half of them located in the loop regions (Figure 2.3 highlighted in yellow). Noteworthy are substitutions in S100A10 at positions F38 and R31, which correspond to conserved leucine and lysine (except S100A11 which corresponds to L36) residues in other S100 proteins respectively. To further analyze the relationship between sequence and structure of these two closely related members of the S100 family, in depth analyses of the three-dimensional structures of S100A11 and S100A10 were conducted.

\subsubsection{Constitutively active conformation of S100A10}

The permanently active form of S100A10 was analyzed using the X-ray crystal structures of S100A10 in complex with annexin A2 peptide (PDB: 1BT6) $[1,2]$ and $\mathrm{Ca}^{2+}$-loaded S100A11 with the N-terminal region of annexin A1 (PDB: 1QLS) [2] in addition to the NMR solution structure of $\mathrm{Ca}^{2+}$-free S100A11 (PDB: 1NSH) [13].

S100A11 undergoes a large calcium-induced conformational change involving the reorientation of helix III in calcium-binding site II [13]. This 'opening' of site II forms a hydrophobic cleft consisting of residues from helices III, IV and the helix II-III linker, and helix I' from the partner protomer essential for interaction with annexins A1 and A2 (Figure 1.10). In contrast, S100A10 adopts an open conformation in the absence of calcium. [13], analogous to that of $\mathrm{Ca}^{2+}$-bound S100A11 (Figure 1.11). 

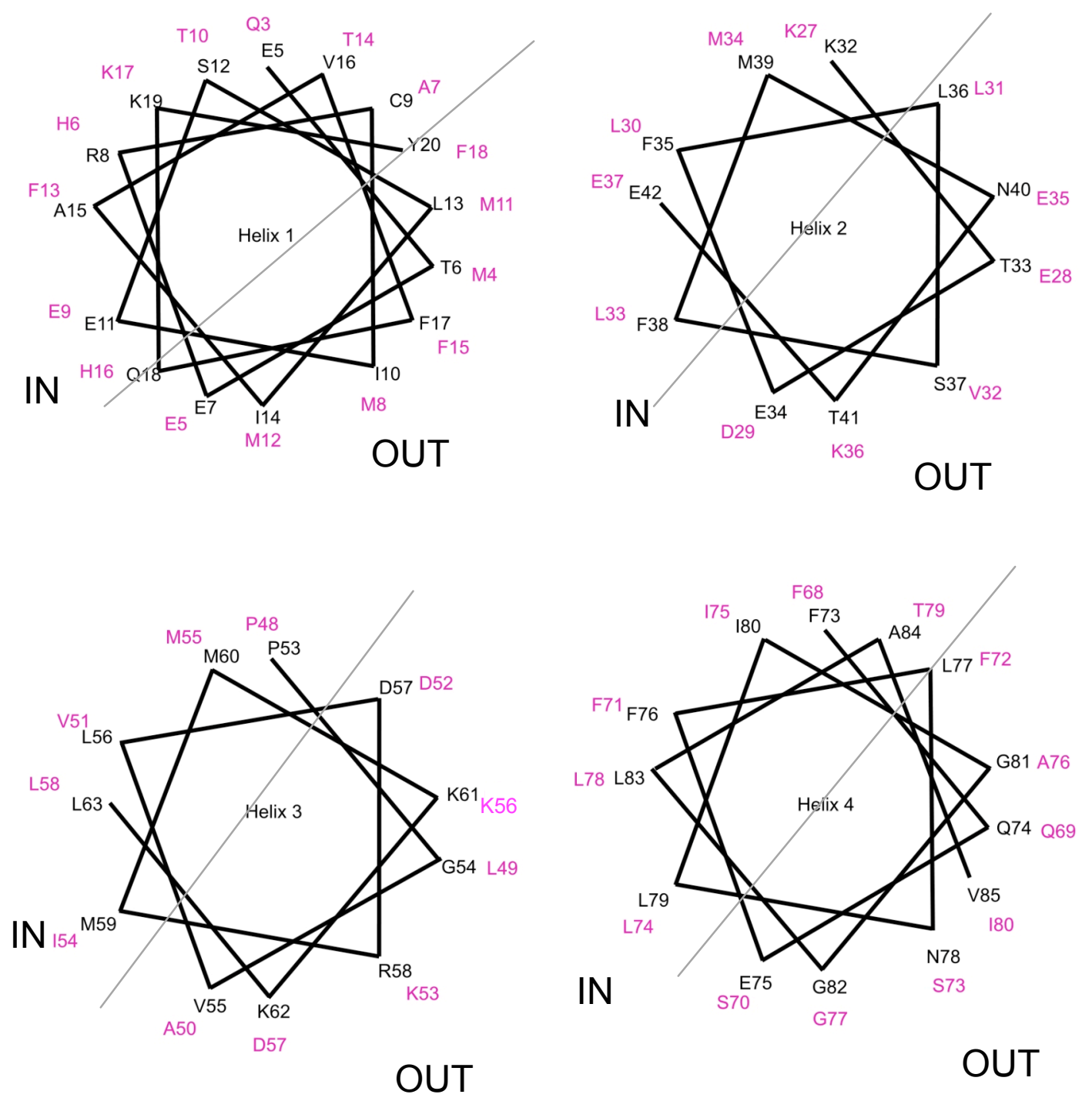

Figure 2.2 Helical wheel representations for S100A10 and S100A11.

Helical wheel projections of S100A11 (black sequence) and S100A10 (red sequence) were performed using the helical wheel applet at http://cti.itc.virginia.edu/ cmg/Demo/wheel/wheelApp.html. The representation illustrates how substitutions in the sequence of S100A10 are concentrated on the outer face of the helices, while the hydrophobic core is more conserved. 
Figure 2.3 shows a comparison of the structures of S100A10 and S100A11. The figure reveals great similarity between the open forms of S100A10 and $\mathrm{Ca}^{2+}-\mathrm{S} 100 \mathrm{~A} 11$ in both EF-hands. A key observation is that the orientation of helices III and IV in $\operatorname{S100A10}\left(\Omega_{\mathrm{III} / \mathrm{IV}}=115^{\circ}\right)($ Table 2.1$)$ highly resembles the calcium-induced opening of helix III/IV in $\mathrm{Ca}^{2+}-\mathrm{S} 100 \mathrm{~A} 11\left(\Omega_{\mathrm{III} / \mathrm{IV}}=113^{\circ}\right)$. In contrast, the interfaces involving helix I are about $15^{\circ}$ more open in S100A10, suggesting that shortening of the N-terminal loop in S100A10 has an effect on the backbone folding of the incoming helix. This leads to helices I/II, I/III and I/IV positioned slightly further apart in S100A10 compared to $\mathrm{Ca}^{2+}$ S100A11, without affecting the dimer interface. Moreover, superposition of the helical regions from S100A10 and $\mathrm{Ca}^{2+}-\mathrm{S} 100 \mathrm{~A} 11$ disclosed low rmsd values between the two proteins and analogous interhelical angles, which confirms their resemblance (Table 2.2). The most significant structural difference between the open forms of S100A10 and the closed conformation in apo-S100A11 involves the positioning of helix III with respect to helix IV, which is arranged in a near perpendicular orientation in $\mathrm{S} 100 \mathrm{~A} 10\left(\Omega_{\mathrm{III} / \mathrm{IV}}=115^{\circ}\right)$ and closer to an antiparallel orientation in apo-S100A11 $\left(\Omega_{\mathrm{II} / \mathrm{IV}}=154^{\circ}\right)($ Figure 1.10 and 2.3). The relationship of helix III to other helices is also altered as shown by large rmsd values involving this helix, as well as large differences in interhelical angles (Tables 2.1 and 2.2). For instance, helix III moves away from helix IV by approximately $40^{\circ}$ in $\mathrm{Ca}^{2+}$ S100A11 compared to apo-S100A11. A similar orientation is seen for this interface in S100A10 $\left(38^{\circ}\right)$, and corresponds to the largest rmsd values between the helix pairs $(>2 \AA)$. 
A

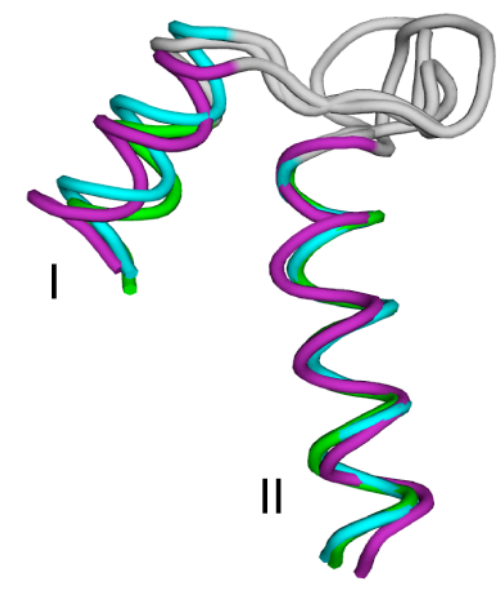

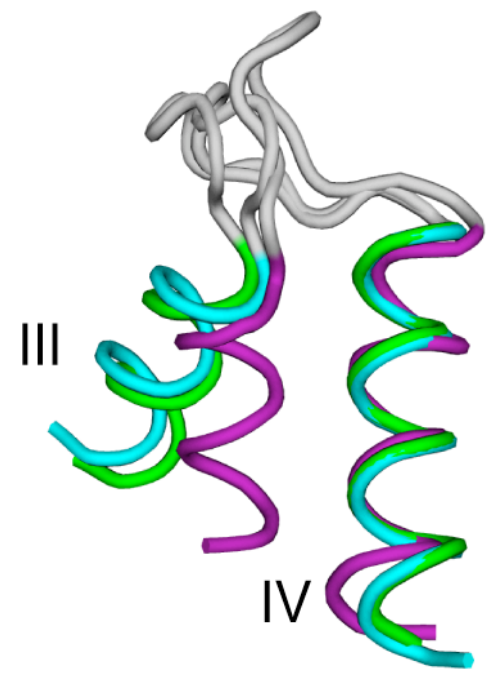

B

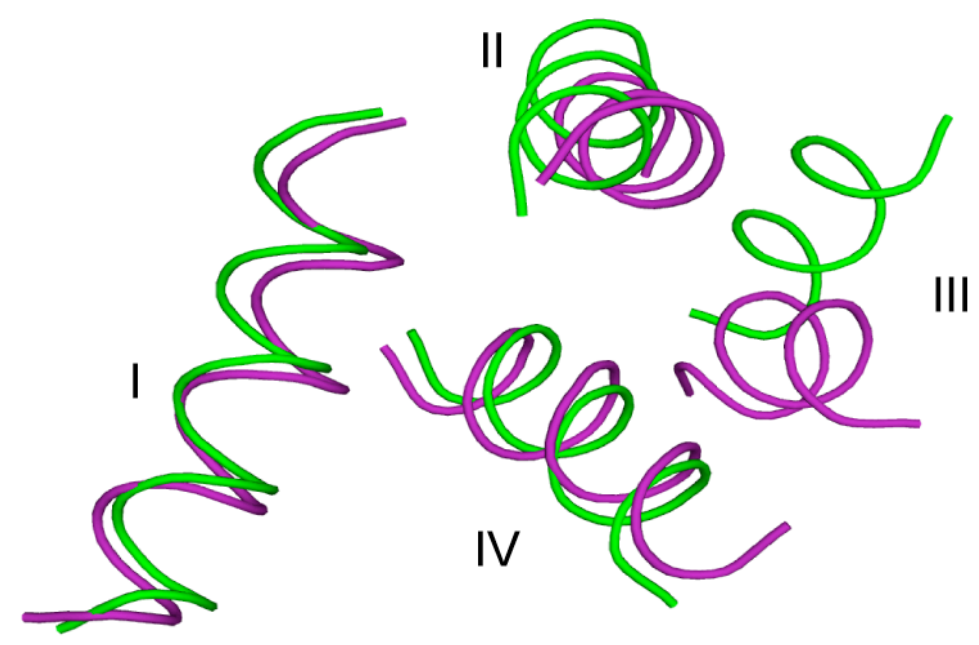

Figure 2.3 Structural comparison between S100A10 and S100A11.

Disposition of EF-hands in S100A10 (green), $\mathrm{Ca}^{2+}-\mathrm{S} 100 \mathrm{~A} 11$ (cyan) and apo-S100A11 (purple) (A). S100A10 shows great similarity to calcium-bound S100A11. The differences between S100A11 and S100A10 are much smaller in magnitude in the Nterminal EF-hand (left). The most significant structural disparity occurs in the C-terminal EF-hand (right) involving the orientation and position of helix III. Orientation of the four helices in S100A10 (green) and apo-S100A11 (purple) (B) shows subtle differences in positions of helix I and IV, which are involved in dimer interface, and considerable repositioning of helix II, and III. Superpositions were made using $\alpha$-carbon atoms. This ribbon representation was drawn using MacPymol (http://delsci.com/macpymol/). 
Table 2.1 Comparison of Interhelical angles ${ }^{\mathrm{a}}$ in S100A10 and S100A11.

\begin{tabular}{|c|c|c|c|c|c|c|}
\hline \multirow[b]{2}{*}{$\begin{array}{c}\text { Helical } \\
\text { Interface }^{b}\end{array}$} & \multirow[b]{2}{*}{$\mathrm{S} 100 \mathrm{~A} 10^{\mathrm{C}}$} & \multirow[b]{2}{*}{$\begin{array}{c}\text { apo- } \\
\text { S100A11 }^{d}\end{array}$} & \multirow[b]{2}{*}{$\begin{array}{c}\mathrm{Ca}^{2+}- \\
\mathrm{S} 100 \mathrm{~A} 11^{\mathrm{e}}\end{array}$} & \multicolumn{3}{|c|}{ Differences $^{f}$} \\
\hline & & & & $\begin{array}{l}\text { S100A10 } \\
\text { vs apo- } \\
\text { S100A11 }\end{array}$ & $\begin{array}{c}\mathrm{Ca}^{2+} \mathrm{S} 100 \mathrm{~A} 11 \\
\text { vs apo- } \\
\mathrm{S} 100 \mathrm{~A} 11\end{array}$ & $\begin{array}{l}\mathrm{S} 100 \mathrm{~A} 10 \\
\text { vs Ca } \\
\mathrm{S} 100 \mathrm{~A} 11\end{array}$ \\
\hline I / II & & & 29 & 29 & -13 & 16 \\
\hline I / III & & -7 & -11 & -21 & 38 & 17 \\
\hline I / IV & 14 & 11 & & 27 & -15 & 12 \\
\hline II / III & 12 & & 71 & -43 & 44 & 1 \\
\hline II / IV & -33 & -41 & -29 & 8 & -12 & -4 \\
\hline III / IV & & 154 & 113 & -39 & 41 & 2 \\
\hline \multicolumn{7}{|c|}{ 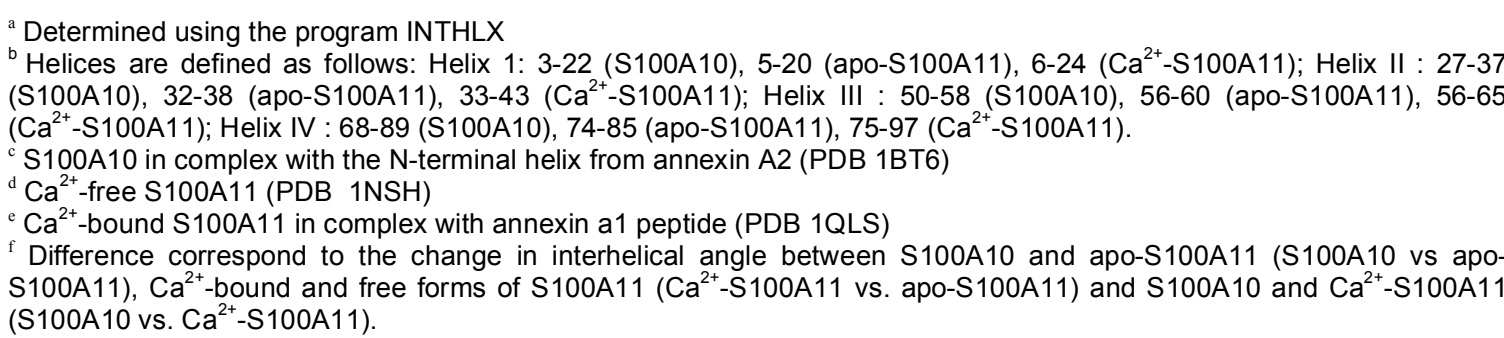 } \\
\hline
\end{tabular}


Table 2.2 RMSD ${ }^{\mathrm{a}}$ on $\mathrm{C} \alpha$ positions from S100A10 and S100A11.

\begin{tabular}{cccc}
\hline $\begin{array}{c}\text { Helical } \\
\text { Interface }\end{array}$ & $\begin{array}{c}\text { S100A10 vs. } \\
\text { apo-S100A11 } \\
(\AA)\end{array}$ & $\begin{array}{c}\mathrm{Ca}^{2+} \mathrm{S} 100 \mathrm{~A} 11^{\mathrm{e}} \mathrm{vs} . \\
\text { apo-S100A11 } \\
(\AA)\end{array}$ & $\begin{array}{c}\text { S100A10 vs. } \\
\mathrm{Ca}^{2+}-\mathrm{S} 100 \mathrm{~A} 11 \\
(\AA)\end{array}$ \\
\hline All residues & 1.61 & 1.54 & 0.85 \\
I / II & 1.12 & 1.02 & 0.55 \\
I / III & 1.98 & 1.88 & 0.68 \\
I / IV & 1.24 & 1.20 & 0.39 \\
II / III & 1.89 & 1.64 & 0.61 \\
II / IV & 1.44 & 1.68 & 0.42 \\
III / IV & 2.57 & 2.84 & 0.57 \\
\hline
\end{tabular}

a'Determined using Swiss-PdbViewer RMSD corresponds to superposition of $C \alpha$ in S100A10 and apo-S100A11 (S100A10 vs. apo-S100A11), Ca ${ }^{2+}$-bound and free forms of S100A11 (Ca ${ }^{2+}-\mathrm{S} 100 \mathrm{~A} 11$ vs. apo-S100A11) and S100A10 and $\mathrm{Ca}^{2+}-\mathrm{S} 100 \mathrm{~A} 11$ (S100A10 vs. $\left.\mathrm{Ca}^{2+}-\mathrm{S} 100 \mathrm{~A} 11\right)$.

${ }^{\mathrm{b}}$ Helices are defined as follows: Helix 1: 3-22 (S100A10), 5-20 (apo-S100A11), 6-24 (Ca $\left.{ }^{2+}-\mathrm{S} 100 \mathrm{~A} 11\right)$; Helix II : $27-37$ (S100A10), 32-38 (apo-S100A11), 33-43 (Ca $\left.{ }^{2+}-\mathrm{S} 100 \mathrm{~A} 11\right)$; Helix III : 50-58 (S100A10), 56-60 (apo-S100A11), 56-65 $\left(\mathrm{Ca}^{2+}-\mathrm{S} 100 \mathrm{~A} 11\right)$; Helix IV : 68-89 (S100A10), 74-85 (apo-S100A11), 75-97 (Ca $\left.{ }^{2+}-\mathrm{S} 100 \mathrm{~A} 11\right)$.

${ }^{c} \mathrm{~S} 100 \mathrm{~A} 10$ in complex with the $\mathrm{N}$-terminal helix from annexin A2 (PDB 1BT6)

${ }^{\mathrm{d}} \mathrm{Ca}^{2+}$-free S100A11 (PDB 1NSH)

${ }^{\mathrm{e}} \mathrm{Ca}^{2+}$-bound S100A11 in complex with annexin a1 peptide (PDB 1QLS) 
In parallel, the relationship of helix III with helices II and I are significantly different with $\sim 40^{\circ}$ shift in S100A10 and $\mathrm{Ca}^{2+}-\mathrm{S} 100 \mathrm{~A} 11$ and rmsd values near $2 \AA$ compared to apo-S100A11. Overall, the superposition of the molecular structures of $\mathrm{Ca}^{2+}$-free, $\mathrm{Ca}^{2+}$-bound S100A11 and S100A10 qualitatively show that helix II and III are the regions of the proteins that presents the largest dissimilarities.

Differences in the open and closed conformations of the S100 proteins involves substantial repacking of the helices that form the protein core as well as a rearrangement of the calcium binding loops. Initially differences in the interhelical faces were studied, followed by analysis of the calcium binding loop regions in S100A10 and S100A11

\subsubsection{Interhelical packing}

The permanently open form of S100A10 was initially compared to apo- and $\mathrm{Ca}^{2+}$ -

S100A11 by calculating $\alpha$-carbon distances in a manner similar to that previously described for Nelson and Chazin (Nelson 1998) [26]. To simplify the matrix obtained from measuring the distance between each pair of $\alpha$-carbon atoms in each protein structure, the analysis were limited to interhelical pairs of residues that are within $8 \AA$ (Figure 2.4). This threshold corresponds to the distance between pairs of residues at the top of helix III and IV (flanking $\mathrm{Ca}^{2+}$ binding loop II) in the calcium-free form of S100A11. As a result of calcium binding or in the active form of S100A10, this distance is greater than the cutoff selected and is used as indicative of significant differences in the relative disposition of the protein backbone. Information about pairs of residues that were within $8 \AA$ were mapped on the structures as shown in Figure 2.4. The atom pair wise comparison showed that helices I and III, and II and IV are not in contact (i.e. $>8 \AA$ ) in 


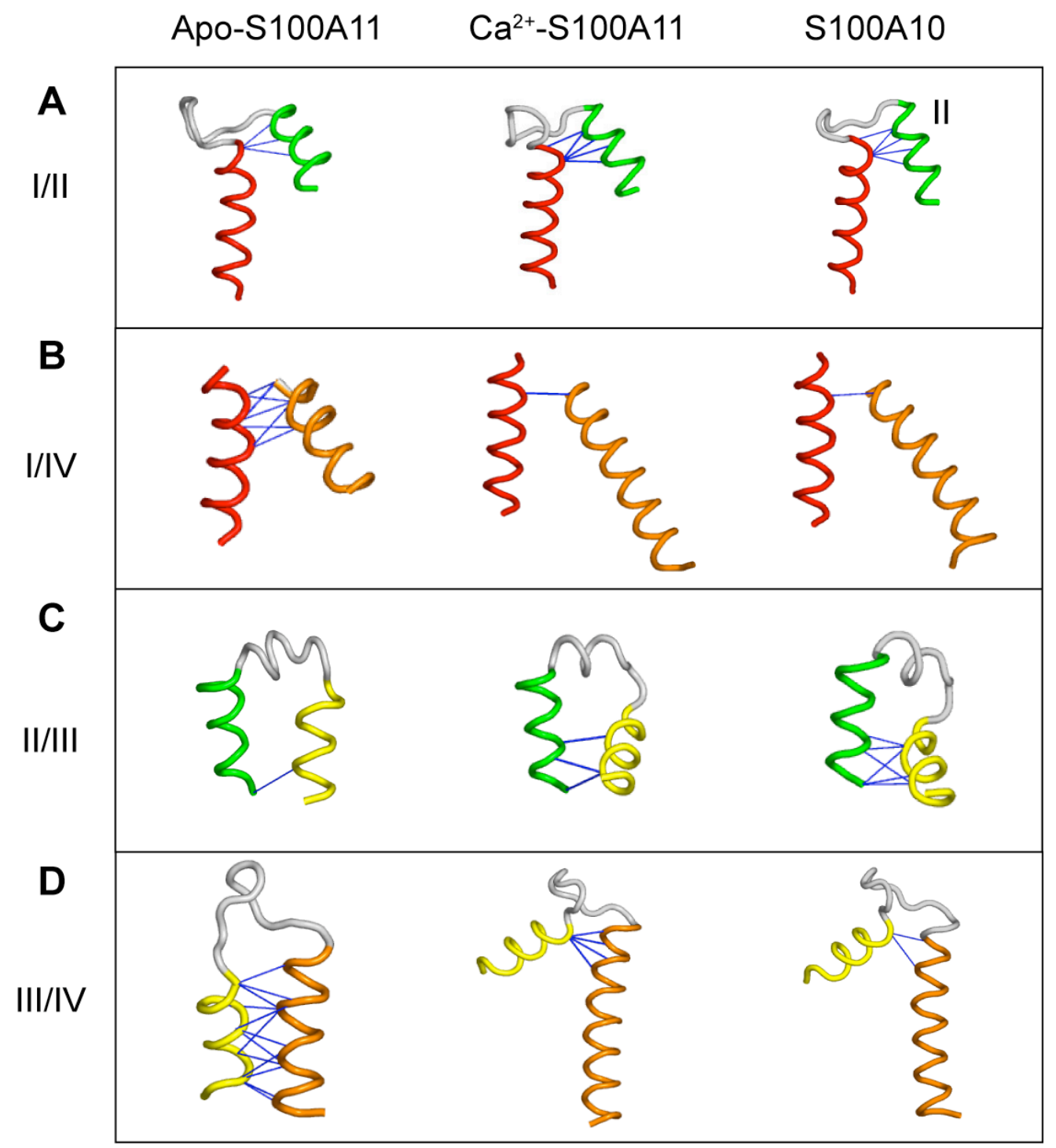

Figure 2.4 $\alpha$-Carbon distances between interhelical faces in S100A11 and S100A10.

Residues pairs in helical interfaces that are within $8 \AA$ are displayed onto the ribbon representation of apo-S100A11 (most representative structure model 12) (left), $\mathrm{Ca}^{2+}$ S100A11 (center) and S100A10 (right) structures as lines between the corresponding $\mathrm{C} \alpha$ atoms. The interfaces shown are (A) I/II, (B) I/IV, (C) (I/III, (D) III/IV. Helices I/III and II/IV are not in contact in any of the proteins in either the closed or the open conformations. Comparison of the closed and open conformations illustrate significant differences in the backbone disposition due to the orthogonal arrangement of helix III (yellow) respect to helix IV (orange) in the open form. Superpositions were made using $\alpha$-carbon atoms. This figure was prepared using MacPymol (http://delsci.com/macpymol/). 
any of the proteins analyzed. Moreover, the helix I/II interface in S100A10 and $\mathrm{Ca}^{2+}$ S100A11 is similar to the apo form, with the ends nearest the calcium-binding loops (top) slightly closer in the apo- forms and the bottom part of the helices further apart (Figure 2.4 A). These two interfaces have similar $\mathrm{C} \alpha$ distances and no large changes in interhelical angles (Table 2.1). Hydrophobic interactions within this interface are highly conserved. One new contact is made between F15 and L33 (F17 and F38 in apo$\mathrm{S} 100 \mathrm{~A} 11)$. The interaction is not possible in apo-S100A11 since F38 is facing in the opposite orientation with the two side chains about $9 \AA$ apart. Further a unique salt bridge is formed between $\mathrm{K} 17$ and $\mathrm{E} 37$ in S100A10 and is also present in the $\mathrm{Ca}^{2+}$-bound S100A11 (K19, E42)

A similar orientation with the C-terminus of helix I and the $\mathrm{N}$-terminus of helix IV in the closed form of S100A11 is revealed by the presence shorter $\alpha$-carbon distances compared with S100A10 and $\mathrm{Ca}^{2+}-\mathrm{S} 100 \mathrm{~A} 11$. Despite this difference, interhelical angles don't show considerably differences and the pattern of contacts between the two helices is very similar in apo- and $\mathrm{Ca}^{2+}-\mathrm{S} 100 \mathrm{~A} 11$ and S100A10. These suggest that the packing of the interhelical interface $\mathrm{I} / \mathrm{IV}$ is essentially conserved amongst these proteins (Figure 2.4B).

As discussed previously the major difference between apo-S100A11 and $\mathrm{Ca}^{2+}$ S100A11 and S100A10 is the orientation of helix III, which allows opening the interface with helix IV. Numerous $\mathrm{C} \alpha$ distances in $\mathrm{Ca}^{2+}-\mathrm{S} 100 \mathrm{~A} 11$ and S100A10 were larger than $8 \AA$ (Figure 2.4D), reflecting the opening of this interhelical face. The analyses indicated that the top of the two helices (near loop II) are slightly closer together in $\mathrm{Ca}^{2+}-\mathrm{S} 100 \mathrm{~A} 11$ 
and S100A10, whereas the bottoms are further apart, with only the top half of the helices being connected. In agreement, the interhelical angle between helices III and IV is about $115^{\circ}$ in the open state and $154^{\circ}$ in the closed apo- S100A11 (Table 2.2) form. Opening of the C-terminal EF-hand is clearly reflected by the disruption of several contacts in this interface in $\mathrm{Ca}^{2+}$-S100A11 and S100A10. For example, in apo-S100A11 helix III and IV are held together by a long stretch of hydrophobic residues, specifically V55, L56 M59, M60 and L63 in helix III and L83, V85, V86, L79 in helix IV, while only three contacts are present in $\mathrm{Ca}^{2+}-\mathrm{S} 100 \mathrm{~A} 11$ between the helices, all of which correspond to residues in the top half of the helix.

On the opposite face of helix III, the interface with helix II is also affected. In S100A10 and $\mathrm{Ca}^{2+}-\mathrm{S} 100 \mathrm{~A} 11$ the C-terminus of helix III comes into close proximity with the N-terminus of helix II (Figure 2.5A). The bottom of the helices are packed more closely in the open conformation adopting a V-shape, which is reflected in the burial of residues L36 and S37 in S100A11 upon calcium coordination. A similar arrangement for interhelical faces II/III, where the bottoms of the helices are closer together and the tops are further apart, is observed for $\mathrm{Ca}^{2+}-\mathrm{S} 100 \mathrm{~A} 6$ [27].

Upon calcium binding or in S100A10, the bottom of helices III and II move closer together disrupting interaction at the top (closer to the loop) of the helices and helix III moves away from helix IV disrupting a series of hydrophobic interactions that position the two helices close together in apo-S100A11 (Figure 2.5B).

In summary, $\alpha$-carbon distances and inter-residue contact analyses have shown that the reorientation of helix III changes the packing of helices III/IV and III/II. Further, 
A

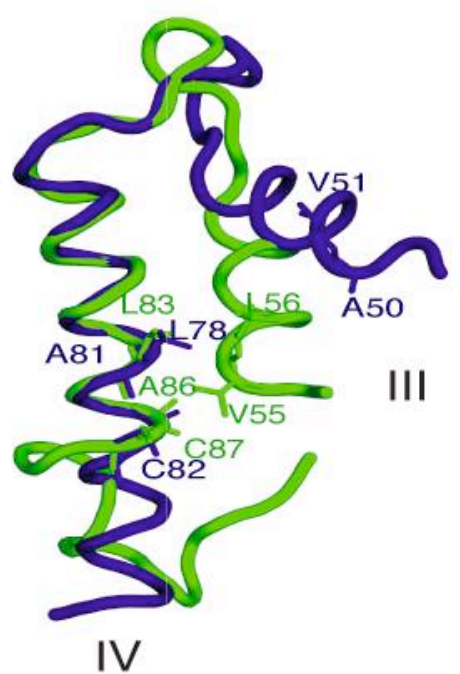

B

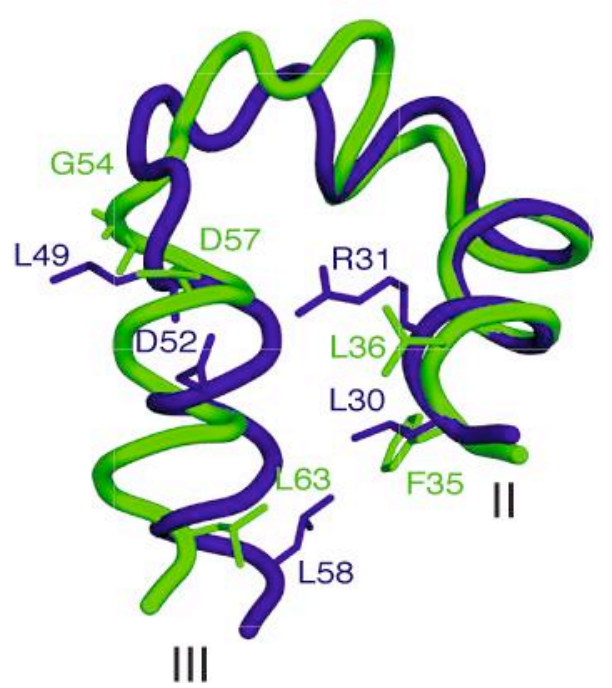

Figure 2.5 Side-chain packing in helix III interfaces from S100A10 and apoS100A11.

Overlay of the three-dimensional structures of S100A10 (purple PDB: 1BT6) and apoS100A11 (green PDB: 1NSH model 12) showing the interfaces between helices III and IV (A) and III and II (B). Hydrophobic interactions between residues in the $\mathrm{N}$-terminus of helix III (V55, L56 in apo-S100A11) and the C-terminus of helix IV (L83, A86 and C87) are disrupted as a result of the repositioning of helix III in S100A10 (A). In S100A10 the C-terminal portion of helix III is closer to the N-terminal region of helix II, allowing a new hydrophobic interaction (L30-L58) and the formation of a hydrogen bond between R31 and D52. 
in apo-S100A11 only the N-terminal half of the helix III shows interactions with helix II (Figure 2.5) and F35 (helix II) faces away from the helix I/II interface in S100A10 and $\mathrm{Ca}^{2+}-\mathrm{S} 100 \mathrm{~A} 11$ breaking contacts with V16 and F17 in helix I present in apo-S100A11. In addition, differences in interhelical packing leads to the formation of two hydrogen bonds in the open form of S100A10 (E37-K17 and R31-D52), that can't occur in apo-S100A11 due to either differences in the orientation of the side chains (E42 and K17 in apoS100A11) or amino acid substitutions (R31 corresponds to L36 in S100A11).

\subsubsection{Surfaces of S100A11 and S100 proteins}

The differences in side chain packing between S100A10 and S100A11 correlate with differences in the protein surfaces. This can be characterized in terms of electrostatic potential and hydrophobic accessible surface area, which is crucial for target recognition in the S100 proteins. As illustrated in Figure 2.6 comparisons of the electrostatic potential of S100A10 and S100A11 reveal a high degree of similarity. Most of the acidic regions exposed in S100A11 correspond to residues in the calcium binding loops, which are substituted for more hydrophilic or neutral residues in S100A10 (Figure 1.13). Moreover, a negatively charged cluster, also observed in S100A6 [28] and S100B [29], delimited by helices III and IV, is formed in $\mathrm{Ca}^{2+} \mathrm{S} 100 \mathrm{~A} 11$ and S100A10. The apo and closed sates of S100A11 present only subtle differences in the distribution of charge residues present in helix III and the linker region.

In addition to the effects in the electrostatic surface of the S100 proteins, the structural rearrangement of helix III upon binding of $\mathrm{Ca}^{2+}$ creates the hydrophobic surface required for target binding. It has been proposed that in $\mathrm{Ca}^{2+}$-signaling proteins, 


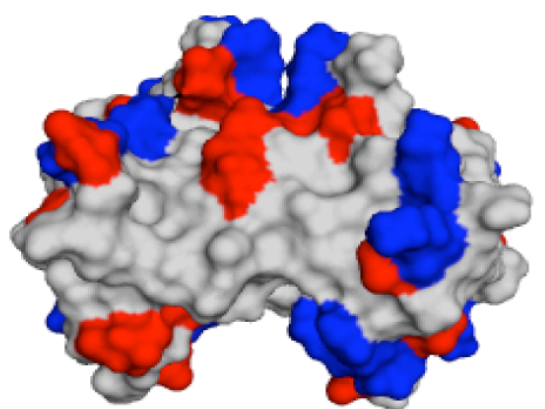

Apo-S100A11

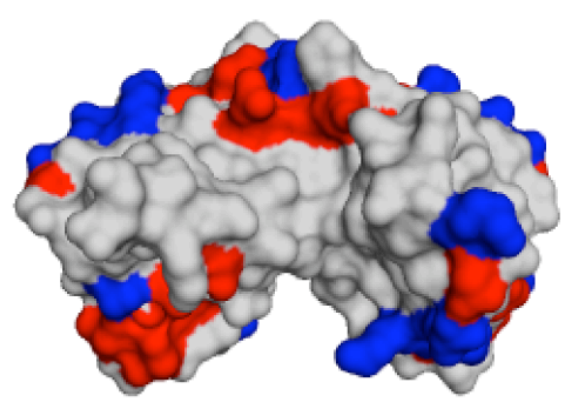

$\mathrm{Ca}^{2+}-\mathrm{S} 100 \mathrm{~A} 11$

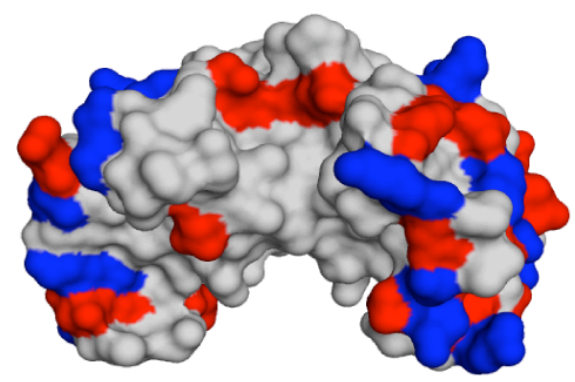

S100A10

Figure 2.6 Electrostatic surface representation of S100A11 and S100A10 proteins.

The electrostatic surface potential is mapped onto the surfaces of each protein. All proteins were aligned using helices I and IV and oriented identically with helix I in front. Red and Blue areas indicate negatively (Glu and Asp) and positively (Lys and Arg) charged residues, respectively. The electrostatic charge distribution shows high similarity amongst the three structures. Surfaces were drawn using MacPymol (http://delsci.com/macpymol/) and PDB accession codes 1NSH (apo-S100A11) [13], 1QLS (Ca2+-S100A11) [2] and 1BT6 (S100A10) [1]. 
stabilizing interactions created by binding of calcium provides the driving force to overcome the energetically unfavourable exposure of hydrophobic residues. Most recently, Ababou and coworkers have shown that substitutions of the buried polar residues with non-polar residues seems to stabilized the closed conformation of the $\mathrm{N}$ terminal of calmodulin, illustrating the importance of a precise balance of solvation energetic to the conformational change in EF-hand proteins [8, 9]. Examination of the surface of S100A10 and comparison to S100A11 (apo- and $\mathrm{Ca}^{2+}$-loaded) structures was used to determine changes of accessible surface area and consequently evaluate solvation effects in regions of S100A10 that could aid to stabilize the open form of S100A10.

The fraction accessible surface area for the side chains of residues in S100A10 and S100A11 (Figure 2.7) showed a large number of residues in helix I and helix IV (T6, C9, I10, L13, I14, V16, F76, L77, I80 and A84) that have more than $80 \%$ of their side chains buried. Most of these residues form part of the dimer interface, which is arranged in an X-type helix bundle. For example, in S100A11 T6 in helix I interacts with L13 and V16 in helix I', and I80 of helix IV'. Comparison of the exposure of side chains in S100A11 and S100A10 show that residues in helix I and II present similar surface exposure amongst the proteins, as judging but the resemblance of the plots in Figure 2.7. However, significant differences are observed on the solvation of the residue at the end of helix I (Y20 in S100A11, F18 in S100A10). Y20 is buried in the open forms of S100A10 and $\mathrm{Ca}^{2+}-\mathrm{S} 100 \mathrm{~A} 11$, and about $30 \%$ exposed in apo-S100A11. An alignment of S100 proteins (Figure 2.1) reveals that Phe is rare at this position and suggests that this residue could potentially have a role in the solvation energetics of S100A10 stabilizing its active 
Helix I

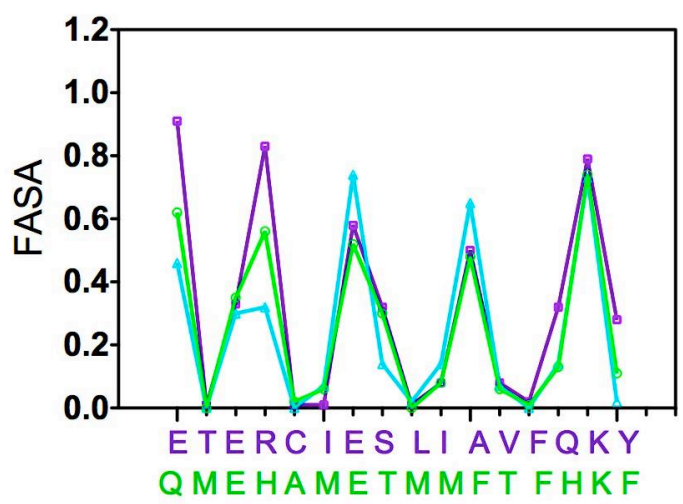

Helix III

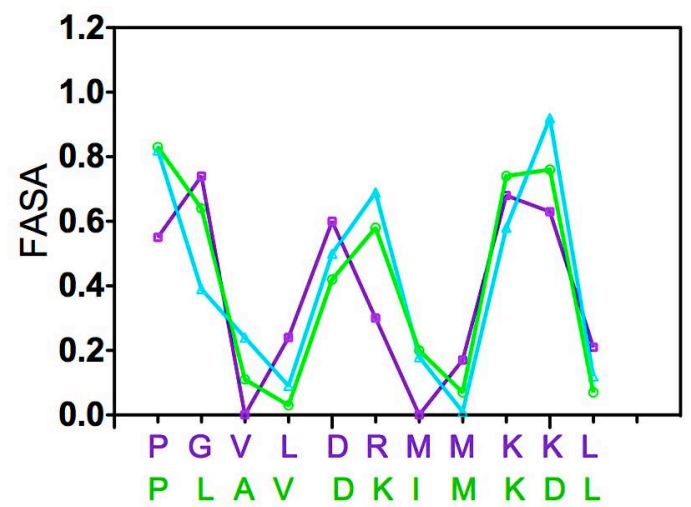

Helix II

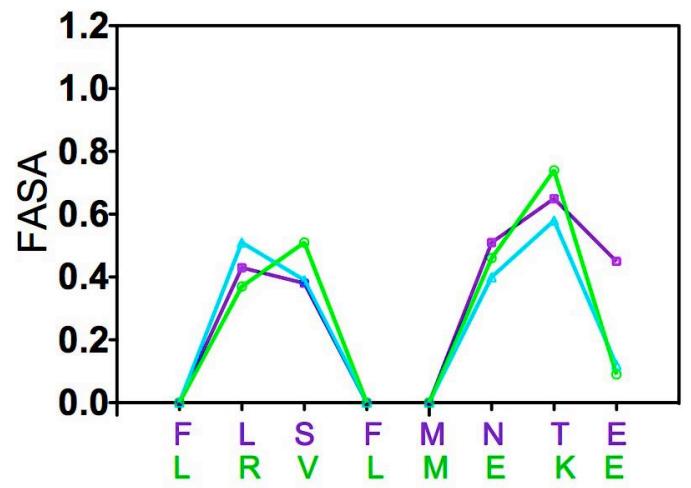

Helix IV

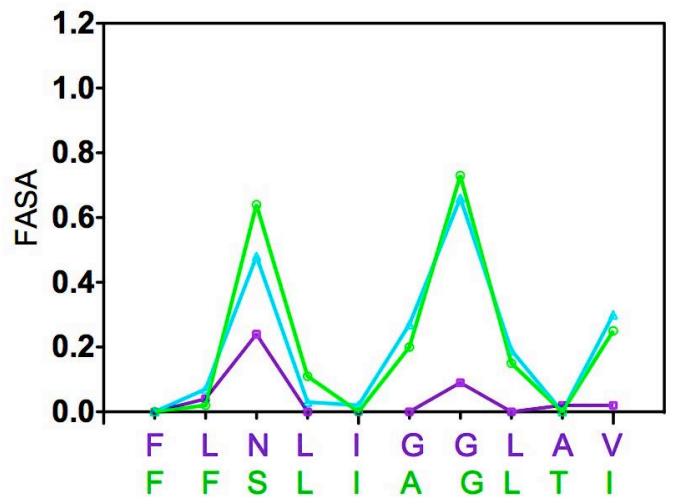

Figure 2.7 Fractional accessible surface are for side chains in S100A10 and S100A11.

The fractional accessible surface area (FASA) for side chains of residues in apo-S100A11 $(-), \mathrm{Ca}^{2+}-\mathrm{S} 100 \mathrm{~A} 11$ (-) and apo-S100A10 (-) were calculated form their PDB coordinates (apo-S100A1: 1NSH model 12; $\mathrm{Ca}^{2+}$-S100A11: QLS and S100A10: 1BT6) using the program VADAR [16]. The accessible surface area for $\mathrm{Ca}^{2+}-\mathrm{S} 100 \mathrm{~A} 11$ and apoS100A10 was calculated after removal of the peptide coordinates from the structures. Residues are plotted as a function of FASA of side chains in each helix. Amino acid sequence for each helix in rabbit-S100A11 (purple) and human S100A10 (green) are shown below the corresponding plots. Helices were defined based on sequence alignment as described in the experimental section of this chapter. 
form. More over, the side chains of Y20 and the corresponding F18 in S100A10 are arranged facing opposite orientations allowing a new hydrophobic interaction with L33 (F35 in apo-S100A11) in helix II, absent in the apo state of S100A11. Interestingly, the hydrophobic L36 in S100A11 is replaced by arginine in S100A10 and it is exposed to the solvent to a similar extent in all the proteins. This residue corresponds to lysine in other S100 proteins (Figure 2.1) and it is unlikely that solvation of this amino acid is the main driving factor that stabilizes S100A10 in the open conformation.

In apo-S100A11 residues in helix IV are more than $70 \%$ buried as a result of the antiparallel orientation of helices III respect to helix IV, present in the closed form of S100A11. Residues L83, A86 and C87, amongst others, become exposed upon calcium binding and in S100A10, and have been shown to be involved in interactions with annexins A1 and A2 [1, 2, 30-32]. The re-orientation of helix III in the open sates of S100A11 and S100A10 also affects the solvation of residues near its C-terminus, particularly, L56, R58 and M59 (V51, K53 and I54 in S100A10). L56 is buried in apoS100A11 and contacts L83 in helix IV. Opening of the interface disrupts this interaction and allows contact with residues in helix II and the linker region (V51-L30 and V52-L42 in S100A10). It also results in R58 and M59 to become exposed to the solvent. R58 corresponds to K53 in S100A10 and forms a unique hydrogen bond with D52 in the linker region that is not present in apo-S100A11. Furthermore, comparison of the primary sequences of S100 proteins (Figure 2.1) shows that Met at position 59 is exclusive of S100A11, and typically corresponds to a hydrophobic Phe or Leu in other S100 members and to Ile in S100A10. The fact that this position can accommodate a wide variety of 
hydrophobic residues suggest that replacement of M59 in S100A11 is unlikely to be the main determinant of the permanently open form of the protein.

The comparisons of fractional accessible surface area described above represent the total exposed surface area. However it is the change in surface exposure between the open and closed forms of S100A11 and S100A10 that corresponds to the structural dissimilarities between them as illustrated in Figure 2.8 and tabulated in Appendix C. Changes in accessible surface area clearly illustrate that the reorientation of helix III and opening of the helix III/IV interface in S100A10 (and $\mathrm{Ca}^{2+}-\mathrm{S} 100 \mathrm{~A} 11$ ) results in the increase surface area exposure of many residues particularly in the C-terminus of protein as discussed previously. Further, the change in side chain accessible surface area calculated for the linker region revealed significant increases at several positions (A45, F46, T47 and D52) in $\mathrm{Ca}^{2+}$-S100A11 (Appendix C) and S100A10 (Figure 2.8 coloured dark orange, and Appendix C) compared to apo-S100A11. These residues are involved in target interaction with annexins A1 and A2. For example the F46 present side chain contacts with L7 and W11 in annexin A1, A45 has contacts with S4, L7 and K8 in annexin A1, and with L7 and S4 in annexin A2. In a similar manner residues F38, F41 and Q45 are highly exposed in S100A10 and show interactions with annexin A2.

Extending the analysis to the loop regions it is observed that side chains of residues at the C-terminal part of the loop I are between 20-40\% exposed in S100A11 and S100A10. Further, the opening of helix III leads to a change in the exposure of residues in site II. In general, residues at the N-terminal of loop II of apo-S100A11 are highly exposed and more than $60 \%$ (negative values Figure 2.8) buried at the C-terminal (positive bars in 


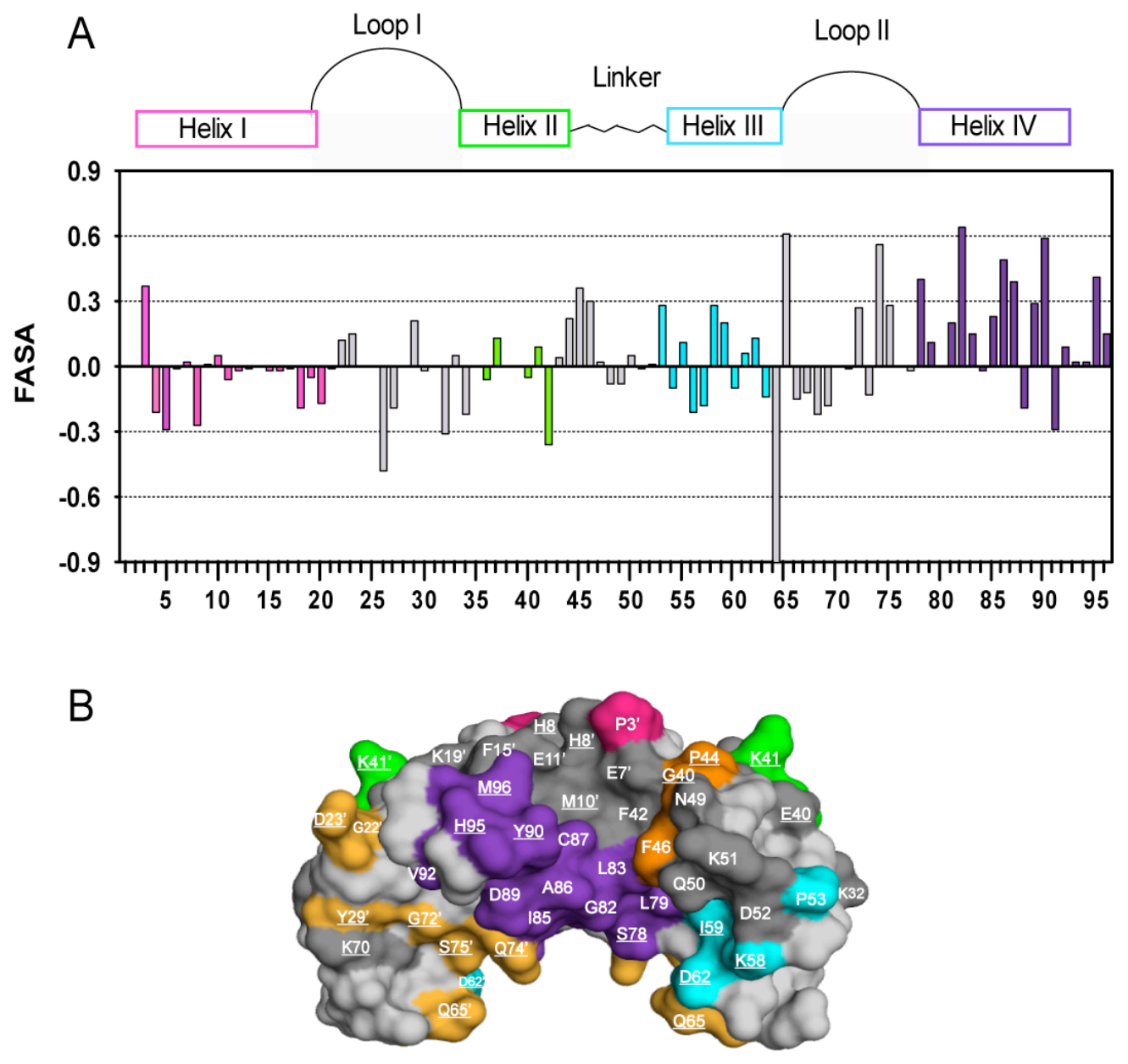

Figure 2.8 Changes in fractional accessible surface area for side chains in S100A10 and apo-S100A11.

(A) Differences in fractional accessible surface area between the structures of apoS100A11 (PDB: 1NSH) [13] and S100A10 (PDB: 1BT6) [1]. The difference corresponds to the surface area for side chains that are exposed in the permanently open conformation of S100A10 (B). Helices are colured as follows: helix I (pink); helix II (green); helix III (cyan) and helix IV (purple). Flexible regions (loops and linker) correspond to filled bars in (A) and coloured orange on the surface representation (B). Residues that are equally exposed in apo-S100A11 and S100A10 are colored grey on (B). Many of the increases in side chain exposure occur in residues from helix IV, III and the linker regions, which form the target-binding patch. To facilitate comparison amino acid numbering (in A and B)corresponds to those of rabbit S100A11 and sequence to the corresponding residue in S100A10. Substitutions in S100A10 compare with S100A11 are underlined on (B). 
Figure 2.8), due to the antiparallel arrangement of helix III respect to helix IV that closes the loop.

In summary differences in side chain surface exposure show large differences in the solvation of residues in helix IV, due to the proximity of the C-terminal portion helix III in apo-S100A11. This arrangement also modifies the solvation of residues in the Cterminus of helix III. More conservative differences occur in helix II and the most similar exposure of side chains is observed for residues in helix I.

\subsubsection{Structural effects of substitutions in S100A10 flexible regions}

As elegantly proven by Chazin and coworkers [10] the conformational change in calcium signaling protein can be modulated by altering the interaction and properties of residues in the protein core. In this work, although a hydrophobic patch similar to that of calmodulin, was successfully exposed upon binding of calcium, the region was covered by the linker that failed to flip out and packed onto the surface through hydrophobic interactions. This suggests that in addition to residues in the protein core, flexible regions could further contribute to the stabilization of the protein in a specific conformation. Further, mutational and structural studies have shown previously that the $\mathrm{Ca}^{2+}$ response can also be controlled by substitutions in the flexible regions of the protein $\left(\mathrm{Ca}^{2+}\right.$-binding loops and linker) $[8,19]$.

To further investigate factors that could control structural differences between S100A10 and S100A11 in the absence of calcium, the comparative analyses for the proteins were extended to the calcium-binding loops and the linker region. These flexible 
sections include about $50 \%$ of the substitutions in the sequence of S100A10 compared to S100A11 and other S100 proteins (Figures 1.10 and 2.1).

In general calcium-binding loops and the linker section are poorly structured and highly dynamic in the $\mathrm{Ca}^{2+}$-free state, as supported by elevated temperature factors in $\mathrm{X}$ ray crystallographic structures, as well as fast amide exchange and broadening effects in the NMR spectra [33]. Increased backbone dynamics in these regions, drastically lower the precision at which the loops are defined in NMR solution structure. For example the NMR structure of apo-S100A11 has an rmsd relative to the mean structure of $1.1 \AA$ and $1.4 \AA$ for the backbone of calcium binding sites II and II respectively, compared to 0.39 $\AA$ for the less well defined helix (helix III). Similar remarks have been reported for S100B and other S100 proteins [34].

Comparative analyses of the EF-hand sites, while relatively limited by the low precision in the structures are critical in these studies due to the large number of substitutions in their sequences. To support the validity of the analyses involving flexible regions of the loops and the hinge between the two EF-hands, x-ray structures of other S100 proteins in their apo- and calcium-bound forms (S100A2 (PDB: 2RGI) [35]; S100A3 (PDB: 1KSO) [36]; S100A6 (PDB: 1K9P) [37] and S100A12 (PDB: 2WCE) [38]) were used in comparative studies, in addition to the NMR structure of apo-S100A11 (PDB: 1NSH) [13], the crystal structure of $\mathrm{Ca}^{2+}-\mathrm{S} 100 \mathrm{~A} 11$ in complex with the Nterminal portion of annexin A1 (PDB: 1QLS) [2] and the complex of S100A10-anenxin A2 peptide (PDB: 1BT6) [1]. In general conclusions were drawn using most representative model of the ensemble of NMR structures reported for apo-S100A11 
(PDB 1NSH, model 12) and interactions were considered existing in apo-S100A11 if they were present in at least $70 \%$ of the structures available (19 models).

\subsubsection{Shortening of the N-terminal loop in S100A10 affects backbone folding}

Coordination of calcium to S100A11 leads to only minor structural changes in site I due to the preformed nature of the loop. The non-canonical EF-hand is considered to be in a calcium-ready state similar to that of calbindin $D_{9 k}$ [39], where positions of calcium coordinating residues are close to the ideal geometry for chelation of the ion. Therefore only minor adjustments to the conformation of the backbone of the loop occurs upon $\mathrm{Ca}^{2+}$ binding without long range structural changes in the fold of the EF-hand I (Figure 2. 3)

The N-terminal loop in S100A10 contains a three-residue gap and uses an aspartate residue at the last coordination position in the loop instead of a conserved glutamic acid used in all other S100 proteins. Shortening of the loop seems to affect backbone folding of the N-terminal EF-hand in S100A10 positioning helix I closer to helix II $\left(\Omega_{\mathrm{I} / \mathrm{II}}=146^{\circ}\right)$ and IV $\left(\Omega_{\mathrm{I} / \mathrm{IV}}=142^{\circ}\right)$ compared with apo-S100A11 $\left(\Omega_{\mathrm{I} / \mathrm{II}}=116^{\circ}\right.$ and $\left.\Omega_{\mathrm{I} / \mathrm{IV}}=115^{\circ}\right)$ and $\mathrm{Ca}^{2+}-\mathrm{S} 100 \mathrm{~A} 11\left(\Omega_{\mathrm{I} / \mathrm{II}}=129^{\circ}\right.$ and $\left.\Omega_{\mathrm{I} / \mathrm{IV}}=127^{\circ}\right)$ (Tables 2.1 and Figures 2.4 and 2.5). As a result the S100A10 N-terminal loop appears narrower compared to other S100 proteins (Figure 2.9). Calcium binding is consequently impaired because the distance between the main chain oxygens involved in coordination of the ion are too close and the aspartate at the coordinating position $-\mathrm{z}$ does not provide the 2 oxygens required to chelate the ion.

S100A7 is another member of the S100 family that also contains an atypical Nterminal EF-hand loop, where the glutamate at position 12 is replaced by a serine and a 
three amino acid deletion is present (Figure 2.9). The arrangement of helix I and II in $\operatorname{S100A10}\left(\Omega_{\mathrm{I} / \mathrm{II}}=146^{\circ}\right)$ is most similar to that observed for $\operatorname{S100A7}\left(\Omega_{\mathrm{I} / \mathrm{II}}=145^{\circ}\right)$ and significantly different from that of S100A11 (Apo: $\Omega_{\mathrm{I} / \mathrm{II}}=116^{\circ}$ and $\mathrm{Ca}^{2+}{ }_{-\mathrm{S}} 100 \mathrm{~A} 11$ $\Omega_{\mathrm{I} / \mathrm{II}}=129^{\circ}$ ). Structural studies have shown that $\mathrm{S} 100 \mathrm{~A} 7$ binds $\mathrm{Ca}^{2+}$ strongly to the canonical EF-hand and unfolds in the absence of the ion [40]. In contrast, S100A10, which lacks the capacity to bind calcium in site II and includes a shortened site I, remains as an stable protein in a permanently active conformation independently of $\mathrm{Ca}^{2+}$ concentrations. This could suggest that shortening of the loop alone is not responsible for the open form in S100A10, since similar folding in site I for S100A7 does not lead to a calcium-like conformation. Substitution of residues in the site I loop and biophysical studies are described in Chapter 3 to test this hypothesis.

Structural comparison of the non-canonical sites in S100 proteins show that the Cterminal portion of the loop is highly structured, constituting the region of the site better defined in the NMR structure of apo-S100A11 (rmsd $0.68 \AA$ for backbone atoms). The last four residues of loop I in S100A10 (L25-D29) show a similar folding compared to that of apo-S100A11 (0.29 $\AA)$ and $\mathrm{Ca}^{2+}-\mathrm{S} 100 \mathrm{~A} 11(0.78 \AA)$ (Figure 2.9). These residues also form part of the exiting helix II and are further stabilized by typical helical hydrogen bonds between residues $\mathrm{i}, \mathrm{i}+4$. In contrast to the end part of the loop, which is well structured and rigid the N-terminal portion of loop I is more flexible and presents a wide range of conformations in solution. Additionally, when the non-canonical loops in S100A10 and $\mathrm{Ca}^{2+}-\mathrm{S} 100 \mathrm{~A} 11$ (or apo-S100A6 and apo-S100A12) are superimposed a 
A

\section{S100A7 TRRD - - - D KI DKPS s100A10 A GD - - KG - YLKTED s100A11 A G K D G H S V T L S K T E}

B

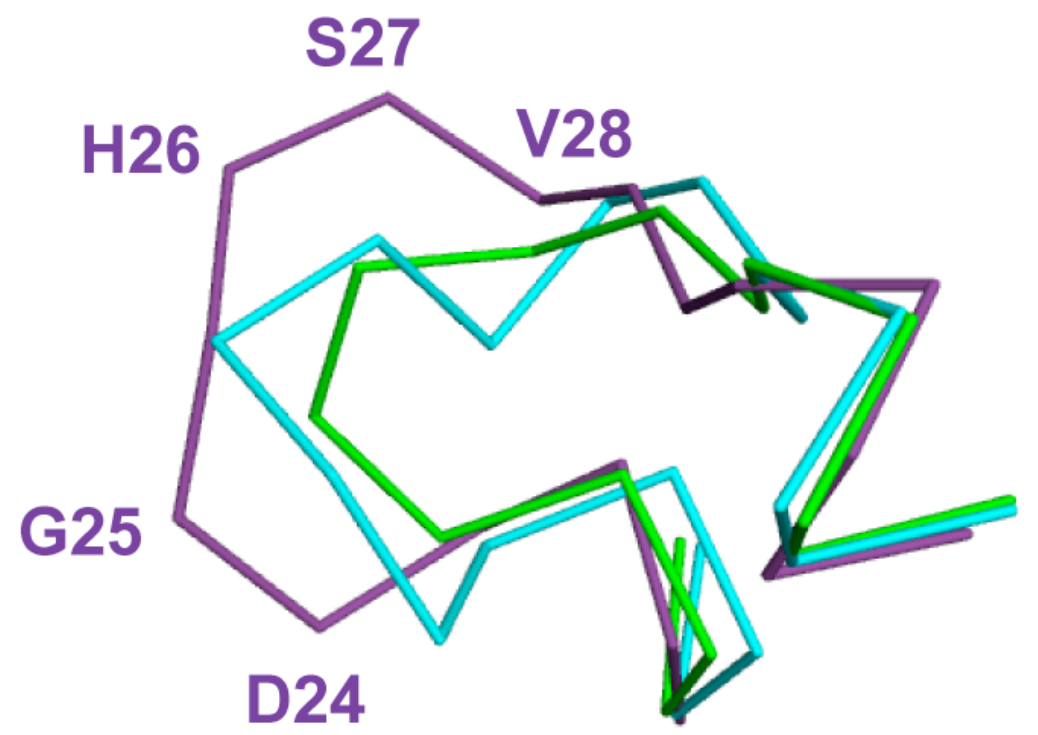

Figure 2.9 Non-canonical EF-hand loop in S100A10.

(A) Sequence alignment of the N-terminal EF-hand loop in S100A7, S100A10 and S100A11, illustrating the three amino acid deletions in S100A7 and S100A10. (B) Structural comparison of site I in S100A10 and S100A11. Overlay of site I in apoS100A11 (purple) (PDB: 1NSH) [13]; $\mathrm{Ca}^{2+}-\mathrm{S} 100 \mathrm{~A} 11$ (cyan) (PDB: 1QLS) [2] and S100A10 (green) (PDB: 1BT6) [1]. Loop I in S100A10 is shortened due to deletions in it sequence and appears narrower than in S100A11. A stretch of residues in the N-terminal portion of loop I seem to overhang compare to a regular 14-residue site I in S100A11. 
stretch of three residues seems to overhang in $\mathrm{Ca}^{2+}-\mathrm{S} 100 \mathrm{~A} 11$, demonstrating that shortening of the loop result in a smaller more compact loop (Figure 2.9). Further, the arrangement of the loop is defined by stabilizing interactions $\left(\mathrm{Ca}^{2+}-\mathrm{O}\right)$ formed upon chelation of the ion, as well as numerous intraloop hydrogen bonds in S100A11. In S100A10, unable to form $\left(\mathrm{Ca}^{2+}-\mathrm{O}\right)$ bonds, the loop is further stabilized through additional hydrogen bond. For example, the side chain of K22 (H26 in apo-S100A11) is oriented toward the inside of the loop allowing three new hydrogen bonds interactions with the main chains of A19, Y24 and the side chain of D29 (Figure 2.10). A unique salt bridge is formed between residues at positions $-\mathrm{y}$ of the two calcium binding sites exclusively in S100A10 (Y24, K65) where S100A10 is the only S100 protein that has a positively charged residue $\mathrm{K} 65$ at this position, corresponding to $\mathrm{E}$ or Q in other S100 members. In most S100 proteins except S100A10, a salt bridge between K32 (apo-S100A11 numbering) and the y position of site II (N/D) is formed. Position y in Site II of S100A10 is replaced by Cys residue that impairs the interaction and the salt bridge is replaced by an interaction with D52 in helix III (Figure 2.10).

\subsubsection{2 "Calcium-ready" folding of loop II in S100A10}

In site II, calcium ligands in S100A11 (N66, E75) are substituted for cysteine and serine residues respectively in S100A10. Five other changes in site II of S100A10 occur compared to S100A11, including L65Q (hydrophobic residue replaced by a hydrophilic residue), S67R and Q70K (introducing new positive charged residues), L71V (smaller hydrophobic residue) and D72G (increased flexibility). 
An examination of site II between S100A10 and S100A11 reveals some obvious similarities and differences best described by splitting the loop into $\mathrm{N}$ - and C-terminal portion at the common sixth position (G64 in S100A10, G71 in S100A11). The Cterminal portion of the loop for the two proteins (KVGFQS in S100A10, QLDFQE in S100A11; Figure 1.8) have a common FQ diad at the tenth and eleventh positions and a branched hydrophobe at the center of the $\beta$-scaffold region (position eight). The ninth position is occupied by glycine in S100A10 and aspartate in S100A11 a position (-X) that is highly variable amongst EF-hand proteins since an intervening water molecule ligates the calcium ion.

In apo-S100A11 the electrostatic repulsion between the side chains of negatively charge residues D64, D68, D72 and E75 presumably forces an extended loop conformation. Upon calcium coordination the positive charge of the ion counterbalances electrostatic repulsions of these residues allowing the side chains to come closer together, rearranging the backbone folding into a more compact conformation. In S100A10 the Nterminal portion of calcium-binding loop II (D59-D63) adopts a conformation similar to that found in the calcium-bound forms of most S100 proteins. However, in x-ray structures of calcium-free S100A2, S100A3 and S100A6 this portion of the loop is positioned in an alternate conformation, while NMR structures show a range of orientations probably due to a greater mobility in solution. The "inversion" of the Nterminal portion of the loop is centered near the Y position. On average, the inversion resulting from calcium binding results in changes in the $\phi, \varphi$ angles of $29^{\circ}$ and $33^{\circ}$ at position $\mathrm{Y}$, and $101^{\circ}$ and $80^{\circ}$ at position $\mathrm{Y}+1$ (Figure 2.11). 

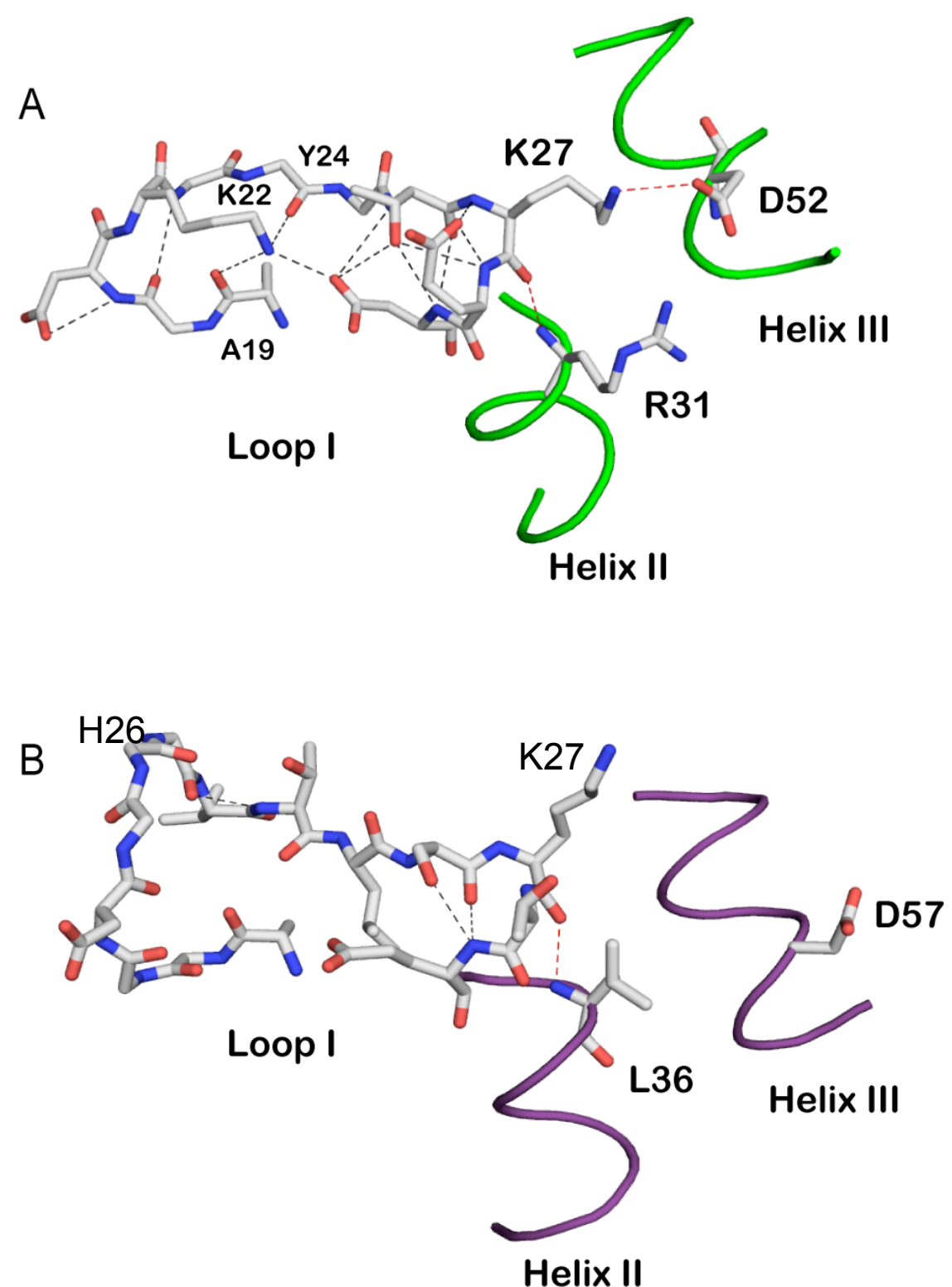

Figure 2.10 Hydrogen-bond network in loop I of S100A10 and S100A11.

Representation of the non-canonical loops of S100A10 (PDB 1BT6 [1]) (A) and apoS100A11 (PDB 1NSH model 12) [13] (B) and helix II and III. The topology of site I in S100A10 is defined and further stabilized for an extensive network of hydrogen binds compared to apo-S100A11. Lys 22 allows formation of additional bonds in S100A10, absent in apo-S100A11. Lys 27 is facing opposite directions in S100A10 and apoS100A11 and only shows interaction with D52 in the open form of S100A10. 


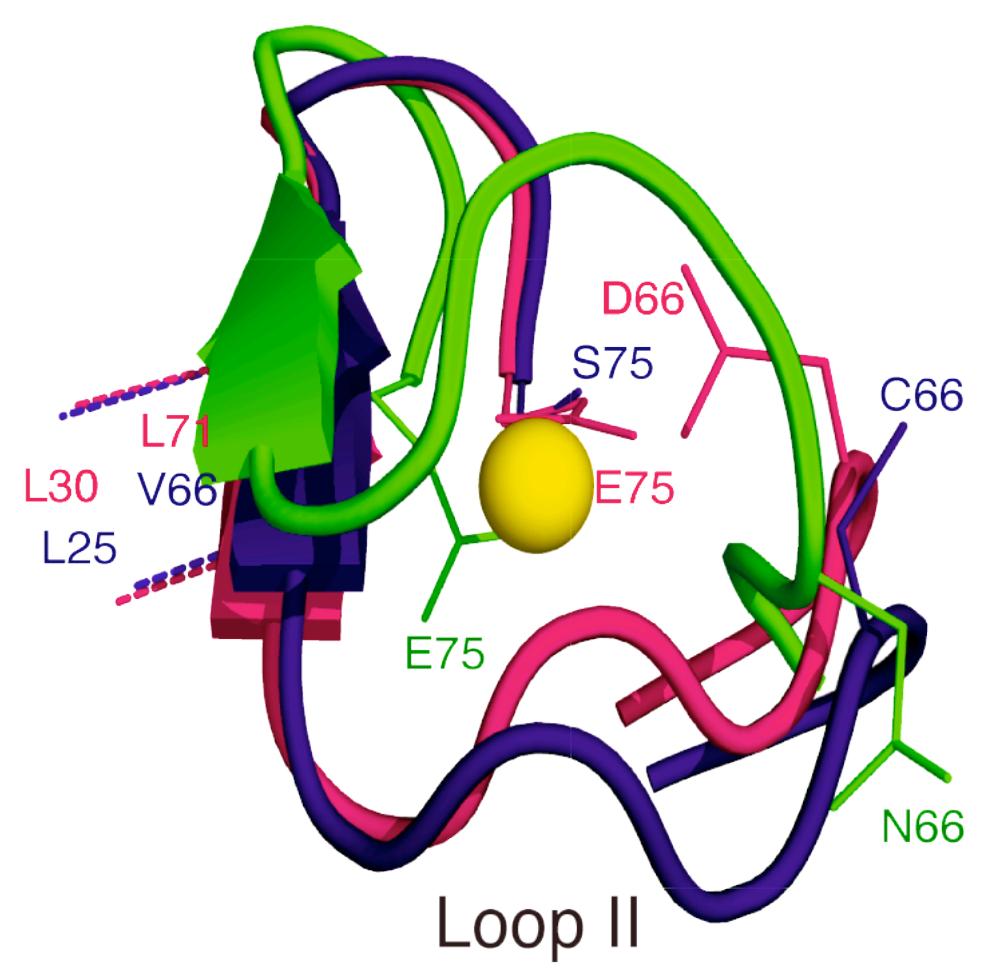

Figure 2.11 Inversion of the canonical loop in $\mathrm{S} 100$ proteins.

Three-dimensional structures of calcium-binding loop II in apo-S100A11 (green), $\mathrm{Ca}^{2+}$ S100A11 (magenta) and S100A10 (purple) showing the inverted conformation of the loop in S100A10 and $\mathrm{Ca}^{2+}-\mathrm{S} 100 \mathrm{~A} 11$, compared to apo-S100A11. Calcium coordinating residues at positions $\mathrm{Y}(\mathrm{D} 66)$ and $-\mathrm{Z}(\mathrm{E} 75)$ are oriented appropriately in $\mathrm{Ca}^{2+}-\mathrm{S} 100 \mathrm{~A} 11$ to allow ligation of the ion, but not in apo-S100A11. Backbone folding in S100A10 and $\mathrm{Ca}^{2+}$-S100A11 permit formation of hydrogen bonds between the main chain of hydrophobic residues (V66, F71) that form part of the short $\beta$-sheet connecting the two calcium-binding loops. These hydrogen bonds are not present in apo-S100A11. PDB codes used 1NSH, 1BT6 and 1QL. This figure was drawn using MacPymol (http://delsci.com/macpymol/). 
Within the site II region it was noted that some interactions were observed in $\mathrm{Ca}^{2+}-\mathrm{S} 100 \mathrm{~A} 11$ and S100A10 that did not exist in apo-S100A11. For example, in S100A10 hydrophobic interactions are observed between F68 in site II and M8, M11 and M12 (F73 with I10, L13 and I14 in S100A10) and a hydrogen bond is formed between the side chain of Q60 (L65 S100A11) and D57 (K62) in helix III. Moreover a striking network of hydrogen bonds in S100A10 seems to stabilize the loop in the calcium-like conformation in S100A10 (Figure 2.12).

In summary, structural analysis of the calcium binding sites in S100A10 show that altered sequences have an effect in the folding of the loop, which is strongly stabilized by numerous hydrogen bonds within each loop and between the two calciumbinding sites. There is precedence in the literature regarding the contribution of hydrogen bonding pattern to the calcium-binding loop conformation [41], and dynamics. It is possible that that the S100A10 open conformation is driven by the arrangements of the loops and the hydrogen bonds formed. Chapter 3 and 4 describe the design and characterization of chimaeric proteins engineered to test this hypothesis.

\subsubsection{Characteristics of the linker region in the open form of S100A10}

The linker region connecting both EF-hands is amongst the most variable for all S100 sequences and has been postulated to regulate target specificity. It also seems to be involved in the calcium-induced conformational change. In S100 proteins, the linker region is long and flexible which facilitates the mobility of helix III and allows a large conformation rearrangement in EF-hand II independent of the pairing EF-hand I 


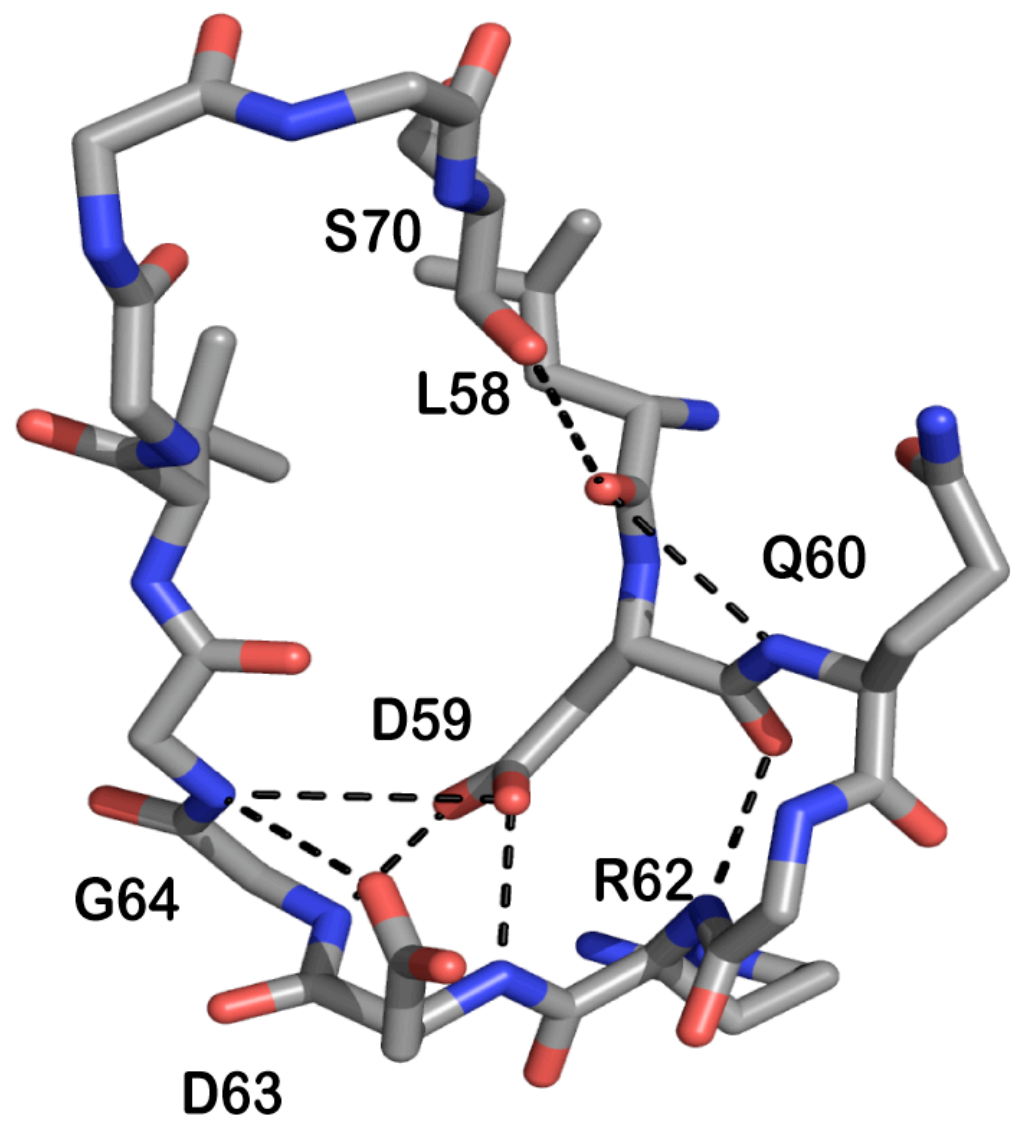

Figure 2.12 Stabilizing hydrogen bond network in loop II of S100A10.

Stick representation of the modified C-terminal EF-hand in S100A10 (PDB 1BT6) [1]. The calcium-like conformation of the C-terminal loop in S100A10 is stabilized by numerous hydrogen bonds, which are represented as dashed black lines in the figure. Representation made using MacPymol (http://delsci.com/macpymol/) 
In contrast, calmodulin and troponin $\mathrm{C}$ have a strong coupling between the EF-hands of each domain leading to a concerted movement of the helices upon $\mathrm{Ca}^{2+}$-binding . Another unique characteristic of the linker region in S100 proteins is the structural presence of a 1.5 turn helix upon binding of calcium, which is also present in S100A10.

Overall interactions of the residues in the linker region are very similar between $\mathrm{Ca}^{2+}$-S100A11 and S100A10. Two residues (F41 and D47) show different arrangements allowing new contacts in the open form of $\mathrm{Ca}^{2+}-\mathrm{S} 100 \mathrm{~A} 11$ and S100A10, missing in apoS100A11. F41 is considerably more exposed in Ca-S100A11 and S100A10, facing opposite to the orientation in apo-S100A11. This promotes new interactions with helix III and IV, including Q50, T47 and F46 with V55, L56 and M59 in helix III of S100A11 respectively (Q45-A50, L42-V51, F41-L54 in S100A10). In addition, upon calcium coordination, repositioning of D52, the residue proceeding helix III, leads to a formation of a new hydrogen bonds between side chain atoms of R58 (D47-K53 in S100A10), that are absent in apo-S100A11 and new hydrophobic interactions between L42 and residues V51 and I54 in helix III (Figure 2.13).

\subsection{Conclusion}

In depth comparative analysis of S100A10 with S100A11 in its apo- and calciumbound forms were conducted aiming to identify differences in sequence and structure that may be responsible for the open conformation of S100A10. The tools used in the studies 


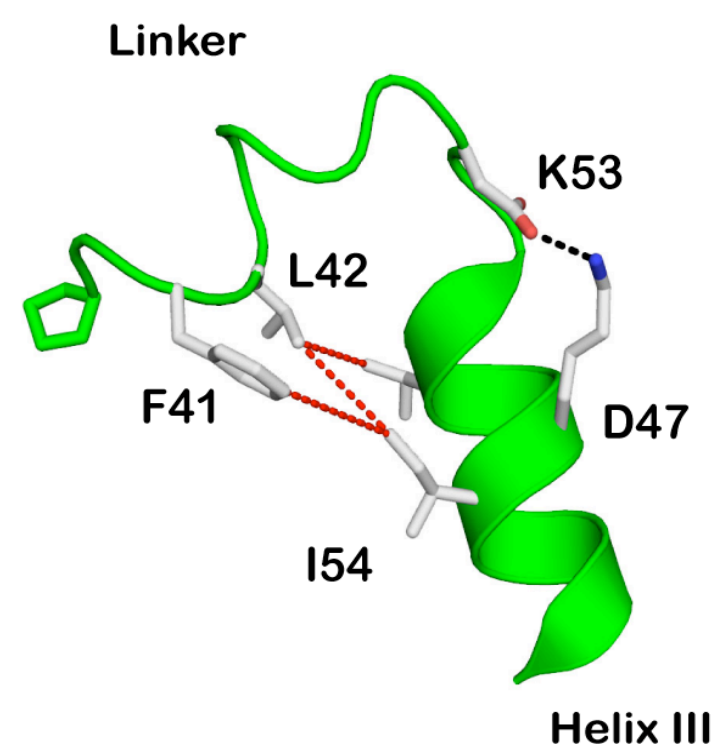

S100A10

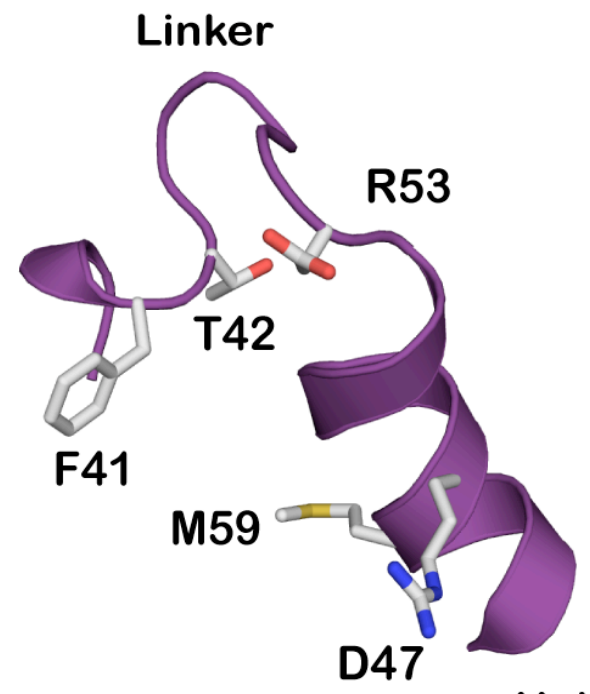

Helix III

Apo-S100A11

Figure 2.13 Interactions in the linker region of S100A10 and apo-S100A11.

Ribbon representations of the linker region and helix III in S100A10 (green) and apoS100A11 (purple). In S100A10 a hydrogen bond (dotted black line) is present between K53 and D47 in helix III. This interaction is not observed in apo-S100A11 as a result of the orientation and substitution of the side chain at the corresponding position. Hydrophobic interactions also occur between residues in the linker and helix III (shown as red dotted lines) that are absent in $\mathrm{Ca}^{2+}$-free S100A11. Figure prepared using MacPymol (http://delsci.com/macpymol/). PDB used 1BT6 [1] and 1NSH [13]. For comparison numbering of the sequences correspond to human S100A10. 
included a combination of carbon alpha distances, inter-residue contact analysis, hydrophobic surface area exposure and three-dimensional structure examination. The key observations included; (a) the first EF-hand loop in S100A10 is three amino acids shorter than the N-terminal loop in S100A11, (b) the calcium-ligands N66 and E75 in the Cterminal loop in S100A11 are replaced by C61 and S70 in S100A10, (c) residues L43 and F46 in the linker region contact amino acids in helices III and IV in S100A11, whereas in S100A10 F38 (L43 in S100A11) shows interactions mainly with residues in helix IV and F41 (F46 in S100A11), is considerably more exposed and is involved in target interaction, (d) a network of hydrogen bonds involving residues in helix III (D52, K53) with those in site I (K27), helix II (R31) and the linker (D47) are present in S100A10, but absent in apo-S100A11, (e) S100A10 is the only protein that has a lysine residue at the Y position in site II, where its side chain forms a unique hydrogen bond with Y24 in site I, and (f) the N-terminus of helix III in apo-S100A11 has multiple contacts with the Cterminus in helix IV, absent in S100A10.

The structural comparisons described in this chapter were the basis to design chimaeric proteins aiming to identify regions responsible for the open conformation in S100A10. 


\subsection{References}

1 Rety, S., Sopkova, J., Renouard, M., Osterloh, D., Gerke, V., Tabaries, S., RussoMarie, F. and Lewit-Bentley, A. (1999) The crystal structure of a complex of p11 with the annexin II N-terminal peptide. Nat Struct Biol. 6, 89-95

2 Rety, S., Osterloh, D., Arie, J. P., Tabaries, S., Seeman, J., Russo-Marie, F., Gerke, V. and Lewit-Bentley, A. (2000) Structural basis of the $\mathrm{Ca}(2+)$-dependent association between S100C (S100A11) and its target, the N-terminal part of annexin I. Structure. 8, 175-184

3 Donato, R. (2001) S100: a multigenic family of calcium-modulated proteins of the EF-hand type with intracellular and extracellular functional roles. Int. J. Biochem. Cell Biol. 33, 637-668

4 Santamaria-Kisiel, L., Rintala-Dempsey, A. C. and Shaw, G. S. (2006) Calciumdependent and -independent interactions of the S100 protein family. Biochem J. 396, 201-214

5 Gerke, V. and Weber, K. (1984) Identity of p36K phosphorylated upon Rous sarcoma virus transformation with a protein purified from brush borders; calciumdependent binding to non-erythroid spectrin and f-actin. EMBO J. 3, 227-233

6 Gerke, V. and Weber, K. (1985) The regulatory chain in the p36-kd substrate complex of viral tyrosine-specific protein kinases is related in sequence to the S-100 protein of glial cells. EMBO J. 4, 2917-2920

7 Glenney, J. R., Jr. and Tack, B. F. (1985) Amino-terminal sequence of p36 and associated p10: identification of the site of tyrosine phosphorylation and homology with S-100. Proc Natl Acad Sci U S A. 82, 7884-7888

8 Ababou, A. and Desjarlais, J. R. (2001) Solvation energetics and conformational change in EF-hand proteins. Protein Sci. 10, 301-312

9 Ababou, A., Shenvi, R. A. and Desjarlais, J. R. (2001) Long-range effects on calcium binding and conformational change in the N-domain of calmodulin. Biochemistry. 40, 12719-12726

10 Bunick, C. G., Nelson, M. R., Mangahas, S., Hunter, M. J., Sheehan, J. H., Mizoue, L. S., Bunick, G. J. and Chazin, W. J. (2004) Designing sequence to control protein function in an EF-hand protein. J Am Chem Soc. 126, 5990-5998

11 Thompson, J. D., Higgins, D. G. and Gibson, T. J. (1994) CLUSTAL W: improving the sensitivity of progressive multiple sequence alignment through sequence weighting, position-specific gap penalties and weight matrix choice. Nucleic Acids Res. 22, 4673-4680

12 Chenna, R., Sugawara, H., Koike, T., Lopez, R., Gibson, T. J., Higgins, D. G. and Thompson, J. D. (2003) Multiple sequence alignment with the Clustal series of programs. Nucleic Acids Res. 31, 3497-3500

13 Dempsey, A. C., Walsh, M. P. and Shaw, G. S. (2003) Unmasking the annexin I interaction from the structure of Apo-S100A11. Structure (Camb). 11, 887-897

14 Guex, N. and Peitsch, M. C. (1997) SWISS-MODEL and the Swiss-PdbViewer: an environment for comparative protein modeling. Electrophoresis. 18, 2714-2723 
15 Drohat, A. C., Amburgey, J. C., Abildgaard, F., Starich, M. R., Baldisseri, D. and Weber, D. J. (1996) Solution structure of rat apo-S100B(beta beta) as determined by NMR spectroscopy. Biochemistry. 35, 11577-11588

16 Willard, L., Ranjan, A., Zhang, H., Monzavi, H., Boyko, R. F., Sykes, B. D. and Wishart, D. S. (2003) VADAR: a web server for quantitative evaluation of protein structure quality. Nucleic Acids Res. 31, 3316-3319

17 Sobolev, V., Sorokine, A., Prilusky, J., Abola, E. E. and Edelman, M. (1999) Automated analysis of interatomic contacts in proteins. Bioinformatics. 15, 327-332

18 Durussel, I., Van Eldik, L. J. and Cox, J. A. (1997) Ion-binding properties of recombinant $\mathrm{S} 100 \beta$ and two derivatives with either an inactivated $\mathrm{Ca}^{2+}$ site II or a normalized $\mathrm{Ca}^{2+}$ site I. Biochim. Biophys. Acta. 1343, 139-143

19 Evenas, J., Thulin, E., Malmendal, A., Forsen, S. and Carlstrom, G. (1997) NMR studies of the E140Q mutant of the carboxy-terminal domain of calmodulin reveal global conformational exchange in the $\mathrm{Ca} 2+$-saturated state. Biochemistry. 36, 34483457

20 Haiech, J., Kilhoffer, M. C., Lukas, T. J., Craig, T. A., Roberts, D. M. and Watterson, D. M. (1991) Restoration of the calcium binding activity of mutant calmodulins toward normal by the presence of a calmodulin binding structure. J. Biol. Chem. 266, 3427-3431

21 Linse, S., Bylsma, N. R., Drakenberg, T., Sellers, P., Forsen, S., Thulin, E., Svensson, L. A., Zajtzeva, I., Zajtsev, V. and Marek, J. (1994) A calbindin D9k mutant with reduced calcium affinity and enhanced cooperativity. Metal ion binding, stability, and structural studies. Biochemistry. 33, 12478-12486

22 Wimberly, B., Thulin, E. and Chazin, W. J. (1995) Characterization of the Nterminal half-saturated state of calbindin D9k: NMR studies of the N56A mutant. Protein Sci. 4, 1045-1055

23 Gifford, J. L., Walsh, M. P. and Vogel, H. J. (2007) Structures and metal-ionbinding properties of the Ca2+-binding helix-loop-helix EF-hand motifs. Biochem J. 405, 199-221

24 Miwa, N., Uebi, T. and Kawamura, S. (2008) S100-annexin complexes--biology of conditional association. FEBS J. 275, 4945-4955

25 Chang, N., Sutherland, C., Hesse, E., Winkfein, R., Wiehler, W. B., Pho, M., Veillette, C., Li, S., Wilson, D. P., Kiss, E. and Walsh, M. P. (2007) Identification of a novel interaction between the $\mathrm{Ca}(2+)$-binding protein S100A11 and the $\mathrm{Ca}(2+)$ and phospholipid-binding protein annexin A6. Am J Physiol Cell Physiol. 292, C1417-1430

26 Nelson, M. R. and Chazin, W. J. (1998) An interaction-based analysis of calciuminduced conformational changes in $\mathrm{Ca} 2+$ sensor proteins. Protein Sci. 7, 270-282

27 Maler, L., Sastry, M. and Chazin, W. J. (2002) A structural basis for S100 protein specificity derived from comparative analysis of apo and $\mathrm{Ca}(2+)$-calcyclin. $\mathrm{J} \mathrm{Mol}$ Biol. 317, 279-290

28 Otterbein, L. R., Kordowska, J., Witte-Hoffmann, C., Wang, C. L. and Dominguez, R. (2002) Crystal structures of S100A6 in the $\mathrm{Ca}(2+)$-free and $\mathrm{Ca}(2+)$-bound states: 
the calcium sensor mechanism of S100 proteins revealed at atomic resolution. Structure. 10, 557-567

29 Smith, S. P. and Shaw, G. S. (1998) A change-in-hand mechanism for S100 signalling. Biochem Cell Biol. 76, 324-333

30 Kube, E., Becker, T., Weber, K. and Gerke, V. (1992) Protein-protein interaction studied by site-directed mutagenesis. Characterization of the annexin II-binding site on p11, a member of the S100 protein family. J Biol Chem. 267, 14175-14182

31 Mailliard, W. S., Haigler, H. T. and Schlaepfer, D. D. (1996) Calcium-dependent binding of S100C to the N-terminal domain of annexin I. J. Biol. Chem. 271, 719725

32 Rintala-Dempsey, A. C., Santamaria-Kisiel, L., Liao, Y., Lajoie, G. and Shaw, G. S. (2006) Insights into S100 target specificity examined by a new interaction between S100A11 and annexin A2. Biochemistry. 45, 14695-14705

33 Marlatt, N. M. and Shaw, G. S. (2007) Amide exchange shows calcium-induced conformational changes are transmitted to the dimer interface of S100B. Biochemistry. 46, 7478-7487

34 Malik, S., Revington, M., Smith, S. P. and Shaw, G. S. (2008) Analysis of the structure of human apo-S100B at low temperature indicates a unimodal conformational distribution is adopted by calcium-free S100 proteins. Proteins. 73, $28-42$

35 Koch, M., Diez, J. and Fritz, G. (2008) Crystal structure of Ca2+ -free S100A2 at 1.6-A resolution. J Mol Biol. 378, 933-942

36 Mittl, P. R. E., Fritz, G., Sargent, D. F., Richmond, T. J., Heizmann, C. W. and Grutter, M. G. (2002) Metal-free MIRAS phasing: structure of apo-S100A3. Acta Cryst. D58, 1255-1261

37 Otterbein, L. R., Kordowska, J., Witte-Hoffmann, C., Wang, C.-L., A. and Dominguez, R. (2002) Crystal structures of S100A6 in the $\mathrm{Ca}^{2+}$-free and $\mathrm{Ca}^{2+}$ bound states: The calcium sensor mechanism of S100 proteins revealed at atomic resolution. Structure. 10, 557-567

38 Moroz, O. V., Antson, A. A., Murshudov, G. N., Maitland, N. J., Dodson, G. G., Wilson, K. S., Skibshoj, I., Lukanidin, E. M. and Bronstein, I. B. (2001) The threedimensional structure of human S100A12. Acta Cryst. D57, 20-29

39 Akke, M., Forsen, S. and Chazin, W. J. (1991) Molecular basis for co-operativity in $\mathrm{Ca} 2+$ binding to calbindin D9k. $1 \mathrm{H}$ nuclear magnetic resonance studies of $(\mathrm{Cd} 2+) 1$ bovine calbindin D9k. J Mol Biol. 220, 173-189

40 Brodersen, D. E., Etzerodt, M., Madsen, P., Celis, J. E., Thogersen, H. C., Nyborg, J. and Kjeldgaard, M. (1998) EF-hands at atomic resolution: the structure of human psoriasin (S100A7) solved by MAD phasing. Structure. 6, 477-489

41 Malmendal, A., Carlstrom, G., Hambraeus, C., Drakenberg, T., Forsen, S. and Akke, M. (1998) Sequence and context dependence of EF-hand loop dynamics. An 15N relaxation study of a calcium-binding site mutant of calbindin D9k. Biochemistry. 37, 2586-2595 


\section{Chapter 3}

\section{DESIGN, SYNTHESIS AND CHARACTERIZATION OF CHIMAERIC S100A11/S100A10 PROTEINS ${ }^{1}$}

\subsection{Introduction}

Unlike all S100 proteins, S100A10 is unable to bind calcium because of a threeresidue gap in its $\mathrm{N}$-terminal $\mathrm{EF}$-hand and substitutions of critical $\mathrm{Ca}^{2+}$ coordinating residues in the C-terminal loop. Despite these deficiencies, S100A10 is locked in a permanently active conformation resembling other $\mathrm{Ca}^{2+}$-bound $\mathrm{S} 100$ proteins and particularly $\mathrm{Ca}^{2+}-\mathrm{S} 100 \mathrm{~A} 11$.

The comparative structural analyses of S100A11 and S100A10 described in Chapter 2 revealed important disparities between the two proteins in their sequences, including substitutions within the S100A10 calcium-binding loops, which impair coordination of the ions. Further, structural differences were also evident between the $\mathrm{Ca}^{2+}$-free forms of these two S100 members, particularly within the interhelical regions involving helix III (II/III and III/IV). The N-terminus of helix III in apo-S100A11 is placed in a near antiparallel arrangement with respect to helix IV forming a strip of hydrophobic contacts absent in the open forms of S100A10 and $\mathrm{Ca}^{2+}-\mathrm{S} 100 \mathrm{~A} 11$.

A complete understanding of the relationship between sequence and structure in S100A10 should be possible by substitutions of positions where larger disparities occur between S100A10 and its closely related partner S100A11. Therefore, generation of

\footnotetext{
${ }^{1}$ Parts of this chapter have been extracted from the published manuscript: Santamaria-Kisiel, L. and Shaw, G.S. 2011. Identification of Regions Responsible for the Open Conformation of S100A10 using Chimaeric S100A11-S100A10 Proteins. Biochem J. 434, 37-48
} 
chimaeric S100A11 proteins incorporating amino acid sequences present in S100A10 was utilized. Considering the long-range contacts that involve helix III/IV and III/II and II and the cooperative nature of the EF-hand system, the approach was to make large modifications in apo-S100A11 and then refine the substitutions based on the characterization of the mutant S100A11 proteins in the calcium-free state.

This chapter details the cloning of seven chimaeric S100A11/S100A10 proteins that incorporated distinct regions of the S100A10 sequence into the S100A11 protein, and the development of novel purification protocols for the proteins engineered. Structural integrity of the chimaeric proteins is also discussed.

\subsection{Materials and Methods}

\subsubsection{Cloning of Chimaeric S100A11/S100A10 proteins}

The pAED4-S100A11 vector (pET-derived) used to express and clone all S100A11 proteins, as well as the GST (glutathione transferase)-fusion vector pGEX-6P1 for expression of S100A10 were gifts from Dr Michael Walsh (Department of Biochemistry and Molecular Biology, University of Calgary, Calgary, Alberta, Canada).

Seven chimaeric S100A11/S100A10 proteins were engineered using site-directed mutagenesis based on the two-stage QuikChange ${ }^{\mathrm{TM}}$ PCR protocol [1]. As a template, the protocol utilized a substituted form of S100A11, where Cys 9 was replaced by Ser 9 to prevent the protein oxidation (referred to as S100A11 in this thesis) observed in the crystal structure of $\mathrm{Ca}^{2+}-\mathrm{S} 100 \mathrm{~A} 11$ [2] and calcium binding experiments [3]. Complementary primers encoding the desired nucleotide substitutions corresponding to the human S100A10 sequence were used to set up two separate primer extension 
reactions (forward and reverse individually) with the DNA template. For a list of primers see Appendix A and for constructs used refer to Appendix B.

In general S100A11 cDNA (100 ng) was combined with $2 \mu \mathrm{M}$ of each particular primer in a $50 \mu \mathrm{L}$ reaction mixture. For the extension reactions $2.5 \mathrm{U}$ of Pfu Turbo DNA polymerase (Stratagene) and the following PCR conditions were used: preheating $95{ }^{\circ} \mathrm{C}$ for $30 \mathrm{sec}, 5$ cycles of $95{ }^{\circ} \mathrm{C}$ for $30 \mathrm{sec}, 55^{\circ} \mathrm{C}$ for $1 \mathrm{~min} ; 68^{\circ} \mathrm{C}$ for $7 \mathrm{~min}$. After cooling down the reactions to $4{ }^{\circ} \mathrm{C}, 50 \mu \mathrm{L}$ of the forward and reverse extended reactions were mixed and subjected to 16 cycles of PCR extension as described above. The product was incubated with 10U of DpnI restriction enzyme (Roche) to digest the parental DNA at 37 ${ }^{\circ} \mathrm{C}$ for $1 \mathrm{~h}$. Undigested PCR product $(10 \mu \mathrm{L})$ was analyzed by gel electrophoresis using $1 \%$ agarose gels and used to transform Escherichia coli XLI-Blue strain cells. Transformed cells were incubated in LB media $(1 \mathrm{~mL})$ for 3 hours and subsequently platted in LB-carbenicillin plates with X-gal and IPTG for white/blue colony selection of positive mutants [4]. The nucleotide sequences of all S100A11/S100A10 constructs were confirmed by DNA sequencing at the Robarts Research Institute (London, Ontario).

Two different sets of S100A11 chimaeric proteins were designed including substitutions and/or deletions into either, a) the calcium-binding loops or b) the helical regions of S100A11, as described below and depicted in Figure 3.1 and Appendix A. S100A11 ${ }^{\text {LI }}$, having the N-terminal calcium-binding loop from S100A10, included three amino acid deletions $(\Delta \mathrm{K} 23, \Delta \mathrm{G} 25$ and $\Delta \mathrm{V} 28)$ and three amino acid substitutions $(\mathrm{H} 26 \mathrm{~K}$, S27G and T29Y) made by PCR-based methods [5] and three additional substitutions (S31T, T33E and E34D) made using the two-stage protocol (above). S100A11 ${ }^{\text {N66C,E75S }}$ 
was constructed using a similar approach and utilized as the base sequence to produce S100A11 ${ }^{\text {LII }}$ where the canonical C-terminal EF-hand loop was replaced for that in S100A10 $\left({ }^{65}\right.$ LNSDGQLDFQE $\left.^{75} \rightarrow{ }^{65} \mathrm{QCRDGKVGFQS}^{75}\right)$. This S100A11 ${ }^{\mathrm{LII}}$ plasmid served as a template to create the $\mathrm{S} 100 \mathrm{~A} 11^{\mathrm{LI}, \mathrm{LII}}$ protein where both calcium-binding sites were replaced as in S100A10 $\left({ }^{21}\right.$ AGDKGYLTKED $\left.{ }^{34},{ }^{65} \mathrm{QCRDGKVGFQS}^{75}\right)$. Proteins $\mathrm{S} 100 \mathrm{~A} 11^{\mathrm{H} 2, \mathrm{H} 3}\left({ }^{35} \mathrm{FLAFMNTE}^{42},{ }^{53} \mathrm{PGVLDRMMKKL}^{63} \rightarrow{ }^{35}\right.$ LRVLMEKE $^{42},{ }^{53}$ PLAVDK IMKDL $\left.^{63}\right)$ and S100A $11^{\mathrm{H} 3, \mathrm{H} 4}\left({ }^{53} \mathrm{PGVLDRMMKKL}^{63},{ }^{76} \mathrm{FLNLIGGLAV}^{85} \rightarrow{ }^{53}\right.$ PLAVDK IMKDL $^{63},{ }^{76}$ FFSLIAGLTI $^{85}$ ), used an S100A11 construct containing the helix III sequence as in S100A10 $\left({ }^{53}\right.$ PLAVDKIMKDL $\left.^{63}\right)$ and introduced either helix II or helix IV substitutions. S100A11 $1^{\mathrm{H} 2-\mathrm{L}-\mathrm{H} 3}$ was generated through substitution $\left({ }^{35}\right.$ FLAFMNTELAAFTKNQKDPGVLDRMMKKL ${ }^{63} \rightarrow{ }^{35}$ LRVLMEKEFPGFLENQKD $^{2}$ PLAVDKIMKDL ${ }^{63}$ ) in the linker region of the S100A1 $1^{\mathrm{H} 2, \mathrm{H} 3}$ containing plasmid.

\subsubsection{Protein Expression and Purification}

All S100A11 proteins (wild-type and S100A11/S100A10 constructs) were overexpressed in Escherichia coli strain BL21(DE3). A fresh single colony of E. coli. BL21(DE3) containing the S100A11 cDNA desired was inoculated in $10 \mathrm{~mL}$ of LB media supplemented with the antibiotic carbeniccilin $(50 \mu \mathrm{g} / \mathrm{mL})$ and grown at $37{ }^{\circ} \mathrm{C}$ overnight with constant shaking. The $10 \mathrm{~mL}$ culture was added to $1 \mathrm{~L}$ (1:100 dilution) of LB media containing the same antibiotic and grown at $37{ }^{\circ} \mathrm{C}$ with agitation to an $\mathrm{OD}_{600}$ of 0.7-0.8. At this point, overexpression was induced by addition of $0.4 \mathrm{mM}$ IPTG and allowed to grow for 4 hours. Cells were harvested by centrifugation, resuspended in $30 \mathrm{~mL}$ of $50 \mathrm{mM}$ Tris- $\mathrm{HCl}, \mathrm{pH} 8.0,5 \mathrm{mM} \mathrm{MgCl}_{2}$ and then lysed using a French pressure 
cell at 20000 psi. Lysates were centrifuged at $95600 \times \mathrm{g}$ for 1 hour at $4{ }^{\circ} \mathrm{C}$ and the supernatants subjected to a variety of purification schemes depending on the properties of the protein. Wild type S100A11, S100A1 $1^{\mathrm{LI}}, \mathrm{S} 100 \mathrm{~A} 11^{\mathrm{H} 2, \mathrm{H} 3}$ and $\mathrm{S} 100 \mathrm{~A} 11^{\mathrm{H} 2-\mathrm{L}-\mathrm{H} 3}$ were purified using a phenyl Sepharose CL-4B matrix (GE Healthcare) as described previously by Rintala et.al [6]. Further purification of S100A $11^{\mathrm{H} 2, \mathrm{H} 3}$ and $\mathrm{S} 100 \mathrm{~A} 11^{\mathrm{H} 2-\mathrm{L}-\mathrm{H} 3}$ were achieved by gravity flow on a Sephadex G-75 column (GE Healthcare) in $25 \mathrm{mM}$ Tris$\mathrm{HCl}, 100 \mathrm{mM} \mathrm{NaCl}, 5 \mathrm{mM}$ DTT and $5 \mathrm{mM}$ EGTA (pH 7.5). Purification of $\mathrm{S} 100 \mathrm{~A} 11^{\mathrm{H} 3, \mathrm{H} 4}$ was completed using a phenyl Sepharose FF column (1 ml, GE Healthcare) equilibrated in $25 \mathrm{mM}$ Tris- $\mathrm{HCl}, 100 \mathrm{mM} \mathrm{NaCl}, 5 \mathrm{mM}$ DTT and $5 \mathrm{mM} \mathrm{CaCl} 2(\mathrm{pH} 7.5)$ and eluted with $25 \mathrm{mM}$ Tris-HCl, $5 \mathrm{mM}$ DTT and $5 \mathrm{mM}$ EGTA (pH 7.5). For chimaeric proteins where the second calcium-binding site was modified cell extracts were dialyzed overnight against running buffer consisting of either $25 \mathrm{mM}$ MES (pH 7.2) $\left(\mathrm{S} 100 \mathrm{~A} 11^{\mathrm{N} 66 \mathrm{C}, \mathrm{E} 75 \mathrm{~S}}, \mathrm{pH} 5.8\right)$ or $25 \mathrm{mM}$ HEPES (S100A11 $\left.{ }^{\mathrm{LII}}, \mathrm{S} 100 \mathrm{~A} 11^{\mathrm{LI}, \mathrm{LII}}, \mathrm{pH} 7.2\right), 1$ mM EDTA and $1 \mathrm{mM}$ DTT. The dialyzed samples were loaded onto a HiTrap SP-XL column (GE Healthcare) equilibrated in the corresponding buffer and elution monitored at $280 \mathrm{~nm}$ with a flowrate of $1 \mathrm{~mL} / \mathrm{min}$. The linear $\mathrm{NaCl}$ gradient used was as follows: 0 min, running buffer $=100 \% ; 50 \mathrm{~min}$, running buffer $=0 \%$, elution buffer $=100 \%$ (as running with $1 \mathrm{M} \mathrm{NaCl}$ addition). Identification of fractions containing S100A11 proteins was monitored by SDS-PAGE (16\% Tris-tricine gels stained with Coomassie blue) where under reducing conditions S100A11 proteins appeared as a single band ( $\sim 10 \mathrm{kDa})$. All purified proteins were extensively dialyzed against $3 \mathrm{mM} \mathrm{KCl}$ and lyophilized for storage. The purity ( $>99 \%$ ) and identity of each protein was confirmed by SDS-PAGE 
and electrospray ionization mass spectrometry (Biological Mass Spectrometry Laboratory, University of Western Ontario).

For uniformly ${ }^{15} \mathrm{~N}$-labeled proteins cells were grown in M9 minimal media supplemented with ${ }^{15} \mathrm{NH}_{4} \mathrm{Cl}(1.0 \mathrm{~g} / \mathrm{L})$ as the sole nitrogen source and the antibiotic carbenicillin $(50 \mu \mathrm{g} / \mathrm{mL})$. A $25 \mathrm{~mL}$ overnight growth of E. coli strain BL21(DE3) containing the desired S100A11 construct in M9 minimal media (above) was inoculated in $1 \mathrm{~L}$ of $\mathrm{M} 9$ media and grown at $37{ }^{\circ} \mathrm{C}$ to an $\mathrm{OD}_{600}$ of $0.7-0.8$. Subsequently, bacterial cultures were induced by addition of $0.4 \mathrm{mM}$ IPTG for 8 hours. Purifications of labeled chimaeric S100A11/S100A10 proteins followed preparative chromatography methodology described above for unlabeled S100A11 constructs.

GST-S100A10 was expressed from bacteria grown in $1 \mathrm{~L}$ of $2 \times \mathrm{YT}$ medium at $37{ }^{\circ} \mathrm{C}$ to an $\mathrm{OD}_{600}$ of 0.6 and expression was induced by the addition of $0.4 \mathrm{mM}$ IPTG. After 4 hours of growth, bacteria were lysed by French pressure cell at 20000psi in 140 $\mathrm{mM} \mathrm{NaCl}, 2.7 \mathrm{mM} \mathrm{KCl}, 10 \mathrm{mM} \mathrm{Na}_{2} \mathrm{HPO}_{4}$ and $1.8 \mathrm{mM} \mathrm{KH}_{2} \mathrm{PO}_{4}$ at $\mathrm{pH}$ 7.3. The lysates were centrifuged at $95600 \mathrm{x} \mathrm{g}$ for $1 \mathrm{~h}$ at $4{ }^{\circ} \mathrm{C}$ and the supernatant was loaded onto a GSTPrep FF 16/10 column equilibrated with lysis buffer. After washing the column until the $\mathrm{OD}_{280}$ returned to baseline, GST-S100A10 was eluted in $50 \mathrm{mM}$ Tris- $\mathrm{HCl}(\mathrm{pH} 8)$ and $10 \mathrm{mM}$ reduced glutathione. Fractions containing GST-S100A10 were pooled and dialyzed overnight against $50 \mathrm{mM}$ Tris- $\mathrm{HCl}(\mathrm{pH} 7.0), 150 \mathrm{mM} \mathrm{NaCl}, 1 \mathrm{mM}$ EDTA and 1 mM DTT. Cleavage of the GST tag was carried out using PreScission Protease (GE Healthcare) in the dialysis buffer according to the manufacturer's directions. After ensuring complete GST cleavage by SDS-PAGE, S100A10 was purified via GSTPrep FF 
16/10 column (GE Healthcare) collecting the flow-through material. The fractions containing S100A10 were pooled and dialyzed against $50 \mathrm{mM}$ Tris- $\mathrm{HCl}(\mathrm{pH} 7.5), 0.2$ $\mathrm{mM}$ EDTA and $0.5 \mathrm{mM}$ TCEP, flash frozen in liquid nitrogen and stored at $-80{ }^{\circ} \mathrm{C}$.

\subsubsection{Oligomeric nature of chimaeric S100A11 proteins}

\subsubsection{Dynamic light scattering experiments}

Oligomerization states of chimaeric S100A11/S100A10 proteins in the absence of calcium were studied by dynamic light scattering (DLS) using a Dynapro DLS photometer (Protein Solutions) equipped with a 827.6nm laser diode. Samples of apoS100A11 proteins (45 $\mu \mathrm{M}$ dimer) at $20{ }^{\circ} \mathrm{C}$ in $20 \mathrm{mM}$ Tris $\mathrm{pH} 7.2,150 \mathrm{mM} \mathrm{NaCl}, 1 \mathrm{mM}$ TCEP and 5mM EDTA were filtered through a $0.02-\mu \mathrm{m} 13 \mathrm{~mm}$ anodisc membrane filter (Whatman) to remove remaining dust particles or solid impurities. Filtered samples (40 $\mu \mathrm{L})$ were loaded into a quartz cuvette and placed into the DynaPro light scattering instrument for data collection. An average of 20 acquisition scans were collected for each of three independently prepared samples with acquisition times set to 3.0 s and the treshold $(\mathrm{S} / \mathrm{N})$ of the readings set at 2.0. Scattered light was collected from a $90^{\circ}$ angle and processed using a DynaPro static light scattering software (DYNAMICS) provided by the manufacturer (Protein Solutions Inc.). A refractive index increment of the sample molecules $(\mathrm{dn} / \mathrm{dc})$ of 0.19 was used, and the partial specific volume was $0.73 \mathrm{~mL} / \mathrm{g}$, a value typical of globular proteins [7]. Time dependent fluctuations in light scattering ( $\mu$ s timescale) due to Brownian motion of S100A11 chimaeric proteins in solutions were analyzed by an autocorrelator inside the DLS instrument to obtain the hydrodynamic raidus $(\mathrm{RH})$ values for each sample and indirectly determine the molecular mass of the 
proteins. The autocorrelator performs a Fourier analysis of the fluctuations, giving the first order autocorrelation function $G(\tau)$, from which the translational diffusion coefficient $\left(\mathrm{D}_{\mathrm{T}}\right)$ is derived as described previously [8].

$$
\mathrm{G}(\tau)=1+\exp \left(-2 \mathrm{D}_{\mathrm{T}} \mathrm{q}^{2} \tau\right)
$$

$\mathrm{D}_{\mathrm{T}}$ values for apo-S100A11/S100A10 proteins were subsequently used to obtain the hydrodynamic radius $\left(\mathrm{R}_{\mathrm{H}}\right)$ from the Stokes-Einstein equation:

$$
\mathrm{R}_{\mathrm{H}}=k_{\mathrm{B}} \mathrm{T} / 6 \pi \eta \mathrm{D}_{\mathrm{T}}
$$

Where $k_{\mathrm{B}}$ is Boltzman constant, $\mathrm{T}$ is temperature in Kelvin, $\eta$ corresponds to the viscosity of the solvent and $\mathrm{R}_{\mathrm{H}}$ is the hydrodynamic radius. Further, estimated molecular weights were calculated from $R_{H}$ values and the sample temperature $\left(20{ }^{\circ} \mathrm{C}\right)$ using standard monomodal curve fit analysis of molecular weights as a function of $\mathrm{R}_{\mathrm{H}}$ of globular proteins, using a DynaPro static light scattering software (DYNAMICS) provided by the manufacturer. (Protein Solutions Inc.). Molecular masses are the means of triplicate readings with background light scattering subtracted.

\subsubsection{Sedimentation Equilibrium Assays}

Sedimentation Equilibrium experiments were conducted in a Beckman Optima XL-A analytical ultracentrifuge using an An60Ti rotor (Beckman, Fullerton, CA). Samples of chimaeric apo-S100A11 (45 $\mu \mathrm{M}$ dimer) dialyzed in buffer containing $20 \mathrm{mM}$ Tris $\mathrm{pH} 7.2,150 \mathrm{mM} \mathrm{NaCl}, 1 \mathrm{mM}$ TCEP and $5 \mathrm{mM}$ EDTA were run in 6-channel cells with epon-charcoal centerpieces, at rotor speeds of 25,000 rpm and 30,000 rpm and 24 ${ }^{\circ} \mathrm{C}$. Absorbance was measured at $280 \mathrm{~nm}$, using 0.002-cm radial steps and averaging over

10 readings. Samples were allowed to equilibrate for 16 hours at the desired speed and 
temperature previous to scanning. The partial specific volume of the proteins was calculated from the inferred amino acid composition, using the freeware program SEDNTERP (Tom Laue, University of New Hampshire). Apparent molecular weights of S100A11/S100A10 proteins were calculated using models constructed in GraphPad Prism $4^{\mathrm{TM}}$.

\subsubsection{Characterization of chimaeric S100A11 proteins}

\subsubsection{Structural integrity}

\subsection{Circular Dichroism}

Folding of all proteins in their calcium-free states were monitored by circular dichroism spectropolarimetry using a Jasco J-810 instrument (Biomolecualr Interactions and Conformations Facility, University of Western Ontario). Typical samples comprised $3.5 \mu \mathrm{M}$ protein (dimer) in $20 \mathrm{mM}$ Tris- $\mathrm{HCl}(\mathrm{pH} 7.2), 10 \mathrm{mM} \mathrm{NaCl}, 0.5 \mathrm{mM}$ TCEP and 5 mM EDTA (10 $\mathrm{mM} \mathrm{CaCl}_{2}$ for $\mathrm{Ca}^{2+}-\mathrm{S} 100 \mathrm{~A} 11$ used as control). For each protein, five scans from $260-190 \mathrm{~nm}(100 \mathrm{~nm} / \mathrm{min}$ at 0.5 increments $)$ were recorded using a $1 \mathrm{~mm}$ path-length cell at $24{ }^{\circ} \mathrm{C}$, averaged and the buffer background was subtracted. Protein concentrations were quantified by using the peak volumes of alanine and leucine residues from duplicate amino acid analyses (Alberta Peptide Institute).

\subsection{NMR Spectroscopy}

The structure and calcium-induced conformational changes in S100A11 chimaeric constructs were evaluated by recording sensitivity-enhanced ${ }^{1} \mathrm{H}-{ }^{15} \mathrm{~N}$ HSQC spectra [9] on uniformly ${ }^{15} \mathrm{~N}$-labeled S100A11 proteins in the absence and presence of calcium. 
Experiments were performed on a Varian INOVA $600 \mathrm{MHz}$ spectrometer equipped with a pulsed field gradient triple resonance probe at $35^{\circ} \mathrm{C}$. All samples contained $10 \% \mathrm{D}_{2} \mathrm{O}$ and $33 \mu \mathrm{M}$ DSS as an internal standard. Protein samples were typically $100 \mu \mathrm{M}$ (dimer) in $20 \mathrm{mM}$ MOPS (pH 7.2), $50 \mathrm{mM} \mathrm{KCl}, 5 \mathrm{mM}$ DTT and either $5 \mathrm{mM}$ EDTA (aposamples) or $10 \mathrm{mM} \mathrm{CaCl} 2\left(\mathrm{Ca}^{2+}\right.$-bound samples). All spectra were processed with NMRPipe [10] software using a $60^{\circ}$ shifted cosine-squared function in the ${ }^{1} \mathrm{H}$ and ${ }^{15} \mathrm{~N}$ dimensions to minimize artifacts in the spectra and analyzed using NMRView [11].

\subsection{Results and Discussion}

\subsubsection{Design of chimaeric S100A11/S100A10 proteins}

Based on the sequence and structural similarities between $\mathrm{Ca}^{2+}-\mathrm{S} 100 \mathrm{~A} 11$ and S100A10 the premise is that minor sequence variations in S100A10, compared to S100A11, allow it to adopt its unique active conformation. To test this hypothesis S100A10 was used as a template to design chimaeric S100A11 proteins that incorporated distinct regions of the S100A10 sequence into S100A11 (Figure 3.1 and Table 3.1) based on the regions where larger dissimilarities in sequence and structure were found between the two proteins as described in the preceding chapter. The assessments showed a three amino acid deletion in the N-terminal EF-hand loop $(\Delta \mathrm{K} 23, \Delta \mathrm{G} 25, \Delta \mathrm{V} 28)$ of S100A10 and substitutions at calcium coordinating positions including the replacement of the invariant glutamate at the $-\mathrm{z}$ coordinating position. Structural and sequence alignment also showed significant substitutions in the canonical EF-hand of S100A10, particularly the replacement of calcium-ligating residues at positions $\mathrm{x}$ and $-\mathrm{z}$ in the coordination sphere (N66C, E75S). 

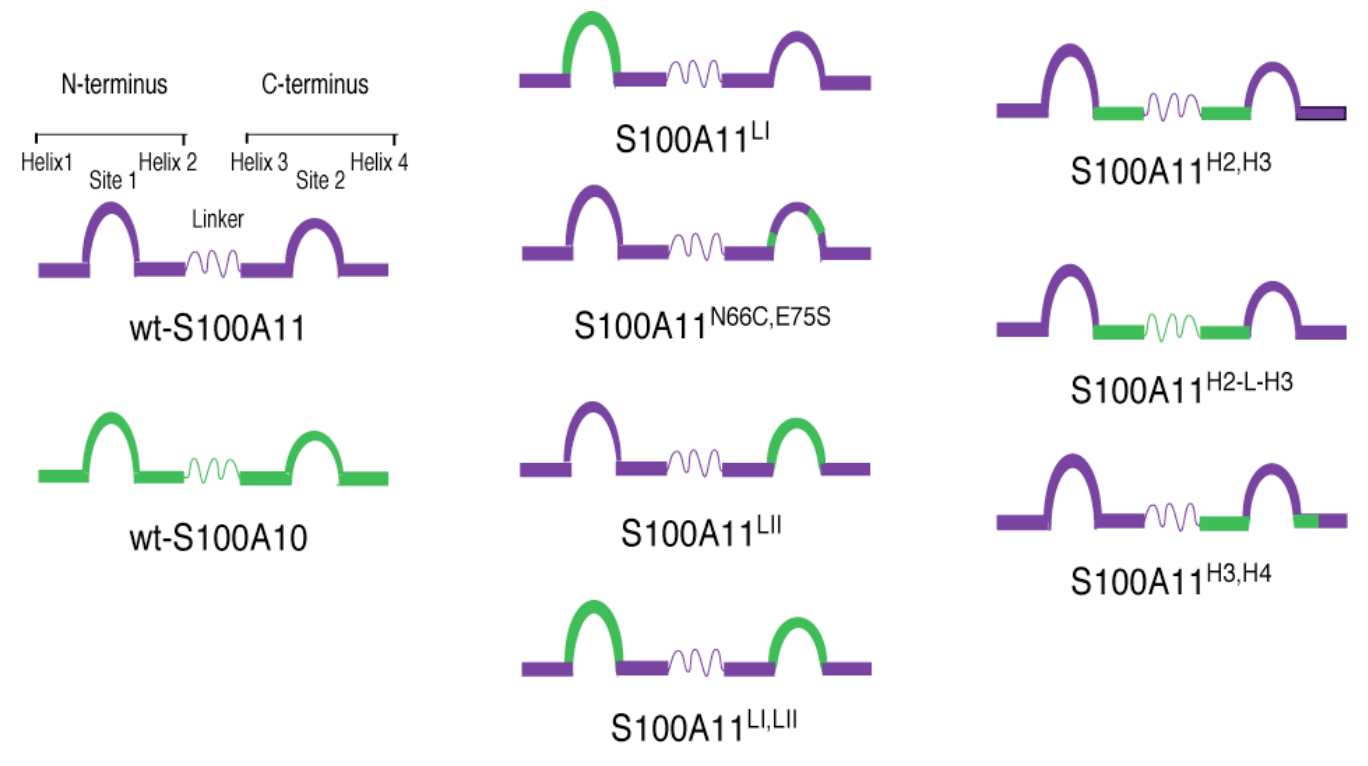

S100A $11^{\text {H2-L-H3 }}$

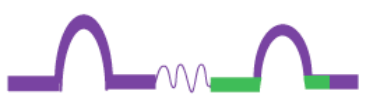

$\mathrm{S} 100 \mathrm{~A} 11^{\mathrm{H} 3, \mathrm{H} 4}$

\section{Figure 3.1 Design of chimaeric S100A11 proteins}

Schematic representation of S100A11 (purple) and S100A10 (green) showing the locations of substitutions in chimaeric S100A11 proteins. Four chimaeric proteins were made incorporating sequences from S100A10 in the calcium-binding sites (center) and three proteins included substitutions from helices II, III, IV and the linker region (right). 
Furthermore, differences in the interhelical packing of helices II/III and III/IV were identified, with the C-terminus of helix III in S100A10 closer to the N-terminus of helix II and the N-terminus of helix III moving away from helix IV exposing a hydrophobic patch for target recognition. In light of these observations, seven chimaeric proteins were designed as outlined in Figure 3.1 and summarized in Table 3.1.

The approach taken was to introduce large substitutions into S100A11 to identify regions that induce a permanently open fold in the protein in the absence of calcium resembling that of S100A10. Subsequently, the region was evaluated based on the characterization and structural analyses and the substitutions fine-tuned to isolate the essential amino acids replacement required.

Site-directed mutagenesis studies on S100 proteins have been reported, particularly exploring the effect of amino acid substitutions in the calcium-binding loops to calcium affinity. For example a complete replacement of the non-canonical loop by a canonical loop in S100B [12] and S100A2 [13] have been reported. Neither the stability nor the structure of the protein in the substituted proteins differed from the wild-type, but significant impacts in $\mathrm{Ca}^{2+}$ and $\mathrm{Zn}^{2+}$ affinities were observed.

To identify the conformational effects of altering the calcium-binding sites of S100A11, four different chimaeric proteins were constructed. The first protein, S100A11 ${ }^{\text {LI }}$, replaced the 14-residue calcium-binding loop in site I with the shorter loop $\left(\mathrm{A}^{19}\right.$ GDKGYLTKED $\left.{ }^{29}\right)$ found in S100A10. A second chimera, (S100A11 ${ }^{\text {N66C,E75S }}$ ) replaced two calcium ligands in site II (Asn66 and Glu75) with residues found in S100A10 (Cys61 and Ser70). As with the S100A11 ${ }^{\mathrm{LI}}$ protein, the second of these 
replacements (Glu75) occurred at the $-z$ position (an invariant glutamate residue in S100 proteins) for calcium co-ordination. Structural analyses of S100 proteins in their apo- and calcium-bound forms shows that the characteristic inversion of the loop occurs at position $\mathrm{Y}$ and $-\mathrm{X}$ (Figure 2.11), which corresponds to the substituted residues in S100A10 (N66C and D72G). These substitutions were introduced in S100A11/S100A10 proteins with an inactive site II to test their impact in the overall fold of S100A11. A substitution of the entire site II loop in S100A11 $\left({ }^{65} \mathrm{LNSDQLDFQE}^{75}\right)$ with that found in S100A10 $\left({ }^{65} \mathrm{QCRDGKVGFQS}^{75}\right)$ was also examined since this protein $\left(\mathrm{S} 100 \mathrm{~A} 11^{\mathrm{LII}}\right)$ had five other changes in site II (Figure 1.13).

Calcium-binding loops are rich in negatively charged acidic residues that are far apart in the calcium-free form of the protein, to avoid destabilizing electrostatic interactions. To coordinate the ion these residues need to come closer together, but the destabilizing interactions are compensated by the presence of the ion. In S100A10 the backbone folding of C-terminal loop resembles that of a $\mathrm{Ca}^{2+}$-bound loop. Since S100A10 is comprised of more neutral residues, it is able to arrange site II much like a compact $\mathrm{Ca}^{2+}$-bound loop without having to overcome destabilizing interactions. The hypothesis to be tested with the chimeras that include substitutions in loop II is that residues in the C-terminal loop of S100A11 allow site II to adopt a conformation similar to that of S100A10 and potentially induce movement of helix III. Finally, the chimeras in site II would evaluate the effect of the increased hydrogen bonding between residues that are substituted in S100A10 (Table 3.1 and Figure 2.12).

Recently it has been proposed that the EF-hand- $\beta$-scaffold structure (residues 6-8) 
and the hydrogen bond formed between the main chains of hydrophobic residues adjacent to residue $-\mathrm{y}$ in paired EF-hands play a crucial role in the calcium-induced conformational change [14]. To test the cooperative structural effects of substitutions in both calcium-binding sites we designed a fourth chimera protein (S100A1 $\left.1^{\text {LI,LII }}\right)$, which combined the substitutions of S100A $11^{\mathrm{LI}}$ and S100A $11^{\mathrm{LII}}$.

In a complementary approach to the one described in this work, the Chazin group used sequences and structural comparisons of calmodulin to elegantly reengineer calbindin $\mathrm{D}_{9 \mathrm{k}}$ as a calcium sensor protein $[15,16]$. The premise of this previous work was that residues governing the calcium-induced conformational change in calmodulin are primarily located in or near the hydrophobic core formed by the four helices [15]. Similarly, to evaluate the importance of the helices towards stabilizing the open form of S100A10, three chimaeric proteins were engineered, focusing on helix III where the largest structural differences exist between apo-S100A11 and S100A10. The contribution of the packing of helices II/III was assessed using a chimaeric S100A11 protein with helices II and III from S100A10 (S100A11 $\left.{ }^{\mathrm{H} 2, \mathrm{H} 3}\right)$. Analyses using $\mathrm{C} \alpha$ distance measurements showed that the C-terminus of helix III is proximal to the N-terminus of helix II (Figure 2.5) in both $\mathrm{Ca}^{2+}-\mathrm{S} 100 \mathrm{~A} 11$ and S100A10, resulting in the burial of Ser37, Asn40, Thr41 and Glu42 (S100A11). The helix III-IV interaction was tested using the chimaeric protein $\mathrm{S} 100 \mathrm{~A} 11^{\mathrm{H} 3, \mathrm{H} 4}$ containing residues from helix III and the first ten residues of helix IV in S100A10. The aim of the S100A $11^{\mathrm{H} 3, \mathrm{H} 4}$ chimaera was to disrupt interactions between residues at the N-terminus of helix III (Val55, Leu56) and residues in helix IV (Leu83, Ala86, Cys87) that occur in apo-S100A11, and are absent in 
Table 3.1 Design of chimaeric S100A11/S100A10 proteins

\begin{tabular}{|c|c|c|c|}
\hline $\begin{array}{l}\text { Chimaeric } \\
\text { Protein }\end{array}$ & $\begin{array}{c}\text { Substitutions } \\
(\text { S100A11/S100A10) }\end{array}$ & Rationale & Hypothesis \\
\hline $\mathrm{S} 100 \mathrm{~A} 11^{\mathrm{LI}}$ & $\begin{array}{l}\Delta \mathrm{K} 23, \Delta \mathrm{G} 25, \Delta \mathrm{V} 28, \\
\mathrm{H} 26 \mathrm{~K}, \mathrm{~S} 27 \mathrm{G}, \mathrm{T} 29 \mathrm{Y}, \\
\mathrm{S} 31 \mathrm{~T}, \mathrm{~T} 33 \mathrm{E}, \mathrm{E} 34 \mathrm{D}\end{array}$ & $\begin{array}{l}\text { Deletions and substitutions in } \\
\text { S100A10 shorten the loop and } \\
\text { impair } \mathrm{Ca}^{2+} \text { coordination. }\end{array}$ & $\begin{array}{l}\text { Shortening of the loop I } \\
\text { modifies } \\
\text { interactions (I/II). }\end{array}$ \\
\hline $\mathrm{S} 100 \mathrm{~A} 11^{\mathrm{N} 66 \mathrm{C}, \mathrm{E} 75 \mathrm{~S}}$ & N66C, E75S & $\begin{array}{l}\text { Mutated acidic } \mathrm{Ca}^{2+} \text { ligating } \\
\text { residues at position } 1 \text { and } 3 \text { in } \\
\text { S100A10. }\end{array}$ & 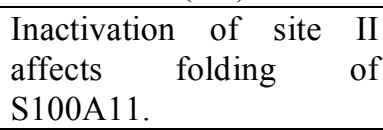 \\
\hline \multirow[t]{3}{*}{$\mathrm{S} 100 \mathrm{~A} 11^{\mathrm{LII}}$} & \multirow[t]{3}{*}{$\begin{array}{ll}\text { L65Q, N66C, S67R, } \\
\text { Q70K, L71V, D72G, } \\
\text { E75S }\end{array}$} & $\begin{array}{l}\text { Site II more hydrophilic in } \\
\text { S100A10. }\end{array}$ & $\begin{array}{lr}\text { Overcoming } & \text { destabilizing } \\
\text { interactions } & \text { amongst } \\
\text { negatively } & \text { charge } \\
\text { residues. } & \\
\end{array}$ \\
\hline & & $\begin{array}{l}\text { N66C D72G positions for loop } \\
\text { inversion. }\end{array}$ & $\begin{array}{l}\text { Loop II is inverted } \\
\text { opening helix III respect } \\
\text { to helix IV. }\end{array}$ \\
\hline & & $\begin{array}{l}\text { Q65-D62 side chain hydrogen } \\
\text { bond; E75S D72G main chain } \\
\text { hydrogen bond in S100A10. }\end{array}$ & $\begin{array}{lr}\text { Increased hydrogen bond } \\
\text { network rabilizes } \\
\text { calcium ready } \\
\text { conformation of the loop. }\end{array}$ \\
\hline \multirow[t]{2}{*}{$\mathrm{S} 100 \mathrm{~A} 11^{\mathrm{LI}, \mathrm{LII}}$} & \multirow[t]{2}{*}{ 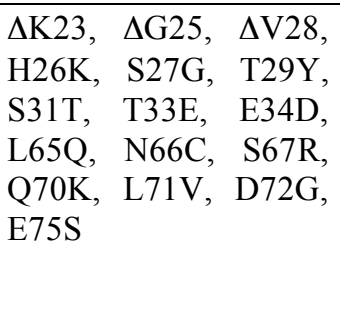 } & $\begin{array}{l}\text { Y29 K70 unique salt bridge } \\
\text { between loops in S100A10. }\end{array}$ & $\begin{array}{l}\text { Cooperative structural } \\
\text { effects of substitutions in } \\
\text { both calcium-binding } \\
\text { loops. }\end{array}$ \\
\hline & & $\begin{array}{l}\text { N66 K32 salt bridge in apo- } \\
\text { S100A11 not in S100A10. }\end{array}$ & $\begin{array}{l}\text { Disrupting hydrogen bond } \\
\text { destabilizes r closed } \\
\text { conformation }\end{array}$ \\
\hline \multirow[t]{2}{*}{$\mathrm{S} 100 \mathrm{~A} 11^{\mathrm{H} 2, \mathrm{H} 3}$} & \multirow[t]{2}{*}{$\begin{array}{l}\text { F35L, L36R, S37V, } \\
\text { F38L, N40E, T41K, } \\
\text { G54L, V55A, L56V, } \\
\text { R58K, M59I, K62D }\end{array}$} & $\begin{array}{l}\text { Bottom of helix II/III packed } \\
\text { more closely in S100A10 } \\
\text { masking L36 and S37. } \\
\text { A55, M59 exposed in } \\
\text { S100A10. } \\
\text { V51L buried in S100A10. }\end{array}$ & Solvation effects \\
\hline & & $\begin{array}{l}\text { R36 hydrogen bond with D52 } \\
\text { in helix III, and E40 in helix II } \\
\text { in S100A10. }\end{array}$ & $\begin{array}{l}\text { R36 has an important role } \\
\text { in open state of S100A10. }\end{array}$ \\
\hline $\mathrm{S} 100 \mathrm{~A} 11^{\mathrm{H} 2-\mathrm{L}-\mathrm{H} 3}$ & $\begin{array}{l}\text { F35L, L36R, } \mathrm{S} 37 \mathrm{~V}, \\
\text { F38L, N40E, T41K, } \\
\text { L43F, A44P, A45G, } \\
\text { T47L, K48E, G54L, } \\
\text { V55A， L56V， R58K, } \\
\text { M59I, K62D }\end{array}$ & $\begin{array}{l}\text { A44 buried in S100A10. } \\
\text { G45, F46 exposed in S100A10 } \\
\text { Q50-A55 hydrophobic } \\
\text { interaction in S100A10 } \\
\text { K58 D52 hydrogen bond } \\
\text { (helix III, linker) in S100A10. }\end{array}$ & $\begin{array}{l}\text { Solvation effects. } \\
\text { Interaction between helix } \\
\text { III and linker stabilizes } \\
\text { opening of helix III. }\end{array}$ \\
\hline $\mathrm{S} 100 \mathrm{~A} 11^{\mathrm{H} 3, \mathrm{H} 4}$ & $\begin{array}{l}\text { G54L， V55A， L56V, } \\
\text { R58K， M59I， K62D, } \\
\text { L77F， N78S， G81A, } \\
\text { V85I }\end{array}$ & $\begin{array}{l}\text { Disruption interactions in the } \\
\text { N-terminus of helix III (V55, } \\
\text { L56) and residues in C- } \\
\text { terminus of helix IV (L83, } \\
\text { A86, C87) in apo-S100A11. }\end{array}$ & $\begin{array}{l}\text { Disruption of contacts } \\
\text { between helix III and IV } \\
\text { leads to opening of the } \\
\text { domain. }\end{array}$ \\
\hline
\end{tabular}


S100A10 (Figure 2.5).

A final chimaeric protein $\left(\mathrm{S} 100 \mathrm{~A} 11^{\mathrm{H} 2-\mathrm{L}-\mathrm{H} 3}\right)$ where the entire helix II-linker-helix III section of S100A11 was replaced by the corresponding section in S100A10 was made because Ala44, Ala45, Phe46, Thr47 and Asp52 become more exposed upon calcium binding in S100A11 and are involved in target interactions with annexin A1 [17]. Substitution of residues in the linker region of S100A11, in addition to changes in helices II and III were designed to establish if the composition of the linker region, along with its interaction with helices II and III (hydrogen bond K58-D52), would modify the conformation of S100A11 in the absence of calcium allowing it to adopt a structure more like that of S100A10. As summarized in Table 3.1 and discussed in the preceding chapter there are significant differences in the exposure of hydrophobic residues in the calcium free form of S100A11 and S100A10. Ababou and coworkers [18] studied the effects of partially buried polar groups in the N-terminal domain of calmodulin. Their findings showed the importance of the specific balance in solvation energetics to conformational change [18].

\subsubsection{Engineering of chimaeric S100A11proteins}

\subsubsection{Cloning of S100A11 mutants}

In order to obtain sufficient quantities of chimaeric S100A11 proteins for biophysical and structural characterization, the cDNA encoding the desired sequences from S100A10 needed to be synthesized and introduced into expression plasmids. 
The initial strategy for inserting substitutions in S100A11 was based on a cassette mutagenesis approach, which would easily allow combination of mutated regions within the protein (i.e. helix pairs). The original plasmid encoding rabbit-S100A11 had been cloned with 5' $X b a \mathrm{I}$ and 3' EcoRI restrictions sites into the pET derived vector pAED4. To utilize cassette mutagenesis, the availability of multiple, unique restriction endonuclease sites are necessary. Therefore, the first step was to introduce three additional restrictions sites within the sequence of S100A11. This would also facilitate the screening of plasmids for the presence of gene mutations in S00A11. Mutations were performed via PCR using overlapping nucleotides encoding sequences for the restriction endonuclease cleavage sites for NsI (ATGCAT), BamHI (GGATCC), StuI (AGGCCT) as shown in Figure 3.2. Using this approach, three amino acids deletions ( $\Delta \mathrm{K} 23, \Delta \mathrm{G} 25$, $\Delta$ V28) and three substitutions (H26K, S27G and T29Y) were introduced in site I via PCR using the appended NsiI and BamHI sites to ligate PCR products. Subsequent attempts to ligate PCR products to incorporate three additional substitutions in site I (S31T, T33E and E34D) were unsuccessful, leading to the design of an alternative methodology to synthesize the chimaeric proteins.

Two single primer PCR reactions (forward and reverse separately) were carried out before performing the standard Quik-Change Mutagenesis ${ }^{\mathrm{TM}}$ Protocol, adjusting the number of cycles according to the extent of the substitutions desired. This method was found to be highly efficient, allowing introduction of multiple mutations, such as in $\mathrm{S} 100 \mathrm{~A} 11^{\mathrm{H} 2-\mathrm{L}-\mathrm{H} 3}$ where 18 residues were substituted using two subsets of complementary primers as described in the experimental section. 


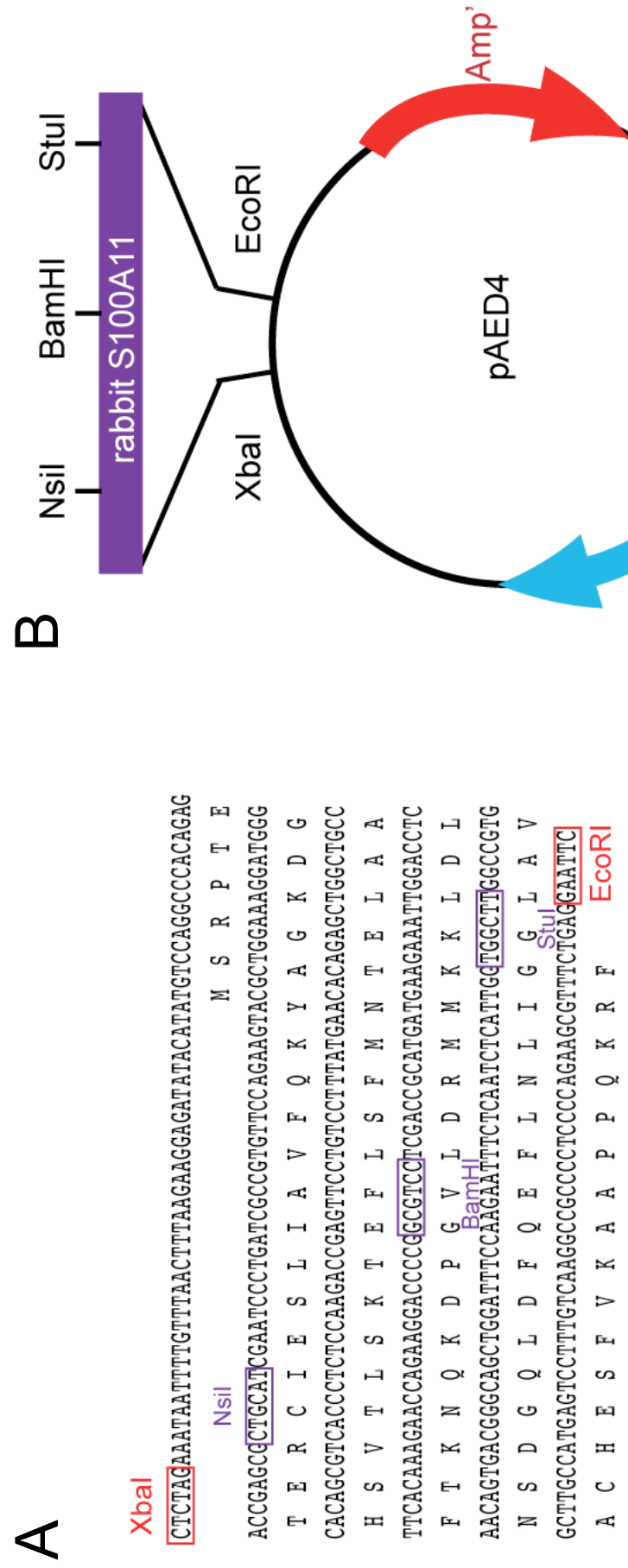

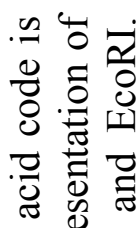

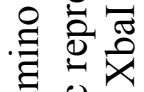

चै.

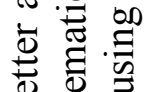

$\dot{\Xi} \tilde{0}$

ङ

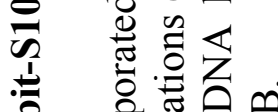

:

-0.

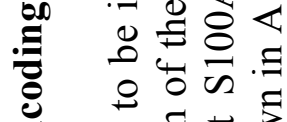

용

瀮

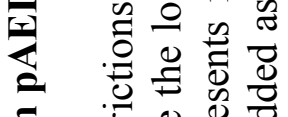

.

产

क छै

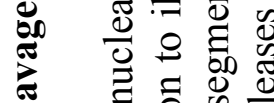

$\frac{\vec{d}}{\mathrm{~J}}$

ঠ

ङ

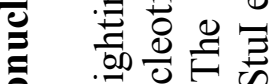

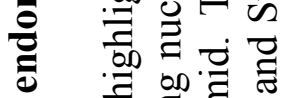

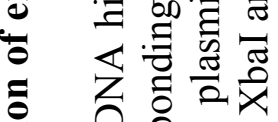

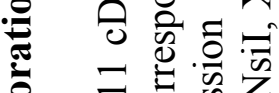

¿ Z

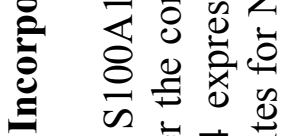

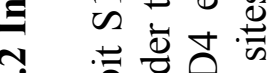

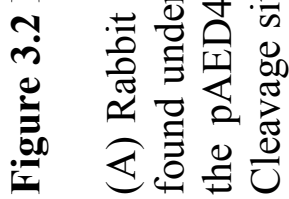


PCR products from the DpnI treated DNA containing the mutation migrated at about $3.3 \mathrm{~kb}$ (Figure 3.3) in 1\% agarose gels. The PCR product size corresponds to the molecular weight of the plasmid, since complementary primers encoding the desired mutation were used to amplify the entire plasmid (Appendix A). As shown in Figure 3.3, quantities of amplification products (as intensity of the bands) differ slightly amongst the chimaeric proteins and are related to the extent of the substitutions introduced. Less intense bands with residual primers (lane 3 in Figure 3.3) are indicative of less effective reactions. For instance, chimaeric $\mathrm{S} 100 \mathrm{~A} 11^{\mathrm{H} 2, \mathrm{H} 3}$ incorporated 10 amino acid substitutions and shows a slight decrease on amounts of amplification product compared to double mutant S100A1 $11^{\mathrm{N} 66, \mathrm{E} 75 \mathrm{~S}}$, where a intense band is seen for the PCR product and remaining primers are not evident.

\subsubsection{Expression and purification of S100A11 proteins}

Upon transformation of the plasmids containing the desired substitutions into E.coli. BL21(DE3), individual chimaeric S100A11 proteins were expressed in LB media and induced using IPTG $(0.4 \mathrm{mM})$ at $37^{\circ} \mathrm{C}$, as described previously for S100A11 [17]. Solubilized extracts of bacterial cultures before and after induction with IPTG were subjected to SDS-PAGE electrophoresis (representative examples shown in Figure 3.4) to monitor induction levels. For all chimaeric S100A11 proteins high degrees of expression were evidenced upon IPTG additions, similar to the bacterial expression of S100A11 (Figure $3.4 \mathrm{~A}$ ), indicating that the substitutions introduced did not have an impact in overexpression of the recombinant S100A11/S100A10 proteins. 


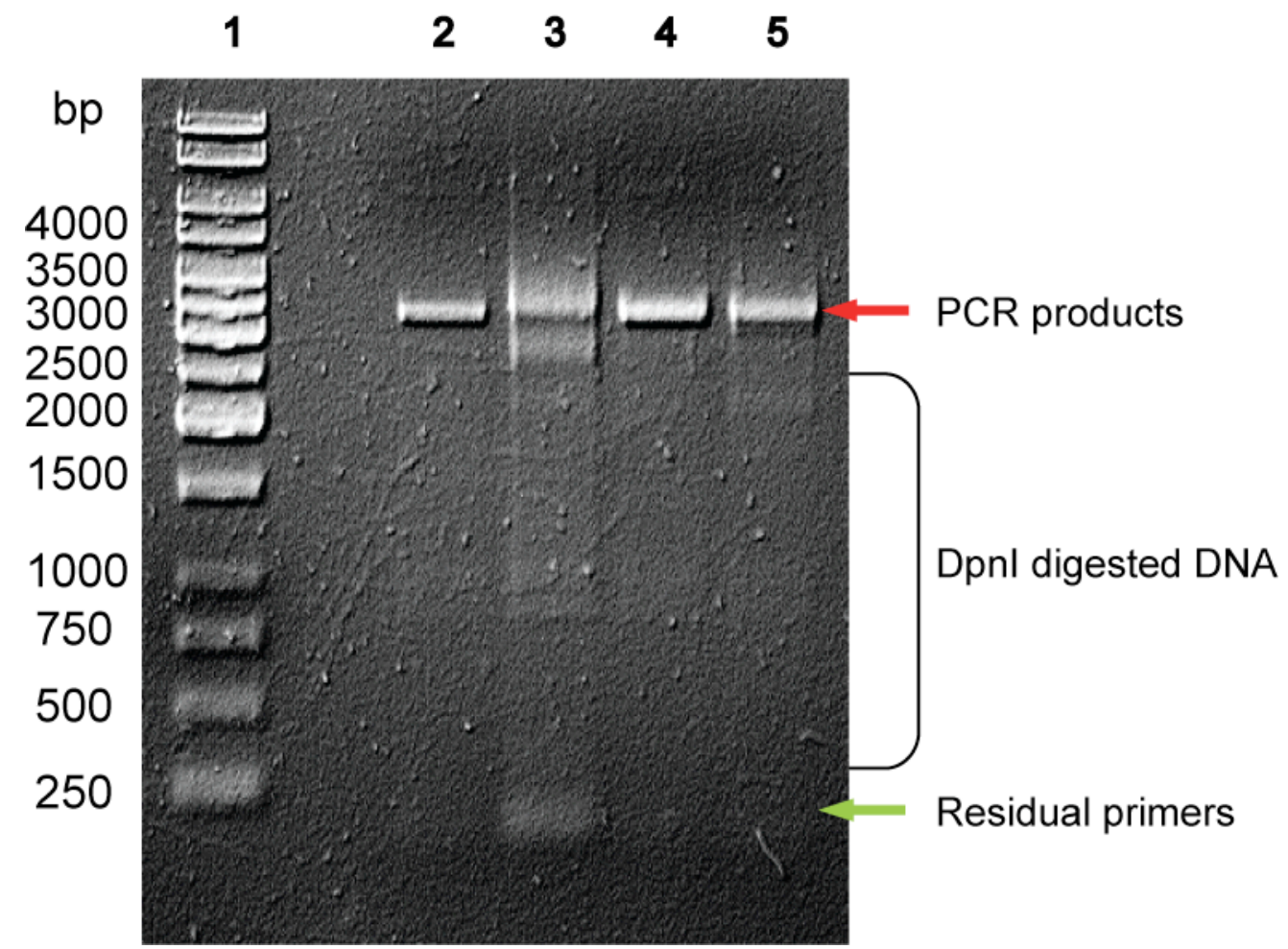

Figure 3.3 PCR product for the synthesis of chimaeric S100A11 proteins.

Polymerase chain reaction (PCR) products for the synthesis of S100A11 ${ }^{\mathrm{N} 66 \mathrm{C}, \mathrm{E} 75 \mathrm{~S}}$ (lane 2) $\mathrm{S} 100 \mathrm{~A} 11^{\mathrm{H} 2, \mathrm{H} 3}$ (lane 3) S100A11 $1^{\mathrm{LI}}$ (lane 4) and S100A11 ${ }^{\mathrm{H} 2-\mathrm{L}-\mathrm{H} 3}$ (lane 5) using a two-step PCR mutagenesis approach. DpnI digested PCR products $(50 \mu \mathrm{L})$ were loaded $(10 \mu \mathrm{L})$ onto a $1 \%$ agarose gels after mutagenesis and stained with ethidium bromide. Incorporation of the desired mutations as PCR products (red arrow) around $3.3 \mathrm{~kb}$ are in agreement with the plasmid size since the protocol used complementary mutagenic primers to amplify the entire plasmid. Lane 1 contains a DNA molecular weight ladder (1kb Fermentas). 
Chimaeric proteins S100A11, S100A11 ${ }^{\mathrm{H} 2, \mathrm{H} 3}, \mathrm{~S} 100 \mathrm{~A} 11^{\mathrm{H} 2-\mathrm{L}-\mathrm{H} 3}, \mathrm{~S} 100 \mathrm{~A} 11^{\mathrm{H} 3, \mathrm{H} 4}$ and S100A11 ${ }^{\text {LI }}$ were purified by hydrophobic interaction chromatography (HIC), taking advantage of the exposure of hydrophobic residue that takes place upon calcium binding. In this method S100A11 proteins in the presence of calcium were bound to a phenyl Sepharose column and after extensive washes with calcium buffer eluted with EGTA containing buffer. As shown in Figure 3.5 for S100A11 ${ }^{\mathrm{H} 2-\mathrm{L}-\mathrm{H} 3}$ (similar results were obtained for $100 \mathrm{~A} 11, \mathrm{~S} 100 \mathrm{~A} 11^{\mathrm{H} 2, \mathrm{H} 3}, \mathrm{~S} 100 \mathrm{~A} 11^{\mathrm{H} 2-\mathrm{L}-\mathrm{H} 3}, \mathrm{~S} 100 \mathrm{~A} 11^{\mathrm{H} 3, \mathrm{H} 4}$ and $\mathrm{S} 100 \mathrm{~A} 11^{\mathrm{LI}}$ ) analysis of the flow through fractions in 15\% SDS-PAGE (Figure 3.5 A lanes 2-4) show that the chimaeric proteins remained bound to the matrix and that elution fractions (lanes $5-12$ ) migrated mainly as single bands around $\sim 10 \mathrm{kDa}$ in agreement with the masses of chimaeric S100A11 proteins (for S100A11 ${ }^{\mathrm{H} 2-\mathrm{L}-\mathrm{H} 3}$ Figure 3.5 lanes 5-12). S100A11 ${ }^{\mathrm{H} 2, \mathrm{H} 3}$ and $\mathrm{S} 100 \mathrm{~A} 11^{\mathrm{H} 2-\mathrm{L}-\mathrm{H} 3}$ proteins were further purified in a Sephadex G-75 column to eliminate high molecular weight contaminants (for final purity of recombinant S100A11 proteins refer to Figure 3.8). Electrospray mass spectrometry was used to confirm the mass and integrity of the chimaeric proteins (Figure 3.5B and Table 3.2). The deconvoluted mass spectrum in Figure 3.5B revealed the presence of one peak at 11325.19 Da, corresponding to the calculated molecular weight of S100A1 $1^{\mathrm{H} 2-\mathrm{L}-\mathrm{H} 3}$ protomer lacking the initiator methionine.

Despite the high degree of expression observed for the chimaeric S100A1 $1^{\mathrm{H} 3, \mathrm{H} 4}$, initial attempts of purification using the protocol described for S100A11, generated $<10 \mathrm{mg}$ of pure protein from a one litre culture (compared to $100 \mathrm{mg} / \mathrm{L}$ of culture for S100A11). Decreases in yield were a result of a stronger interaction of the protein with 


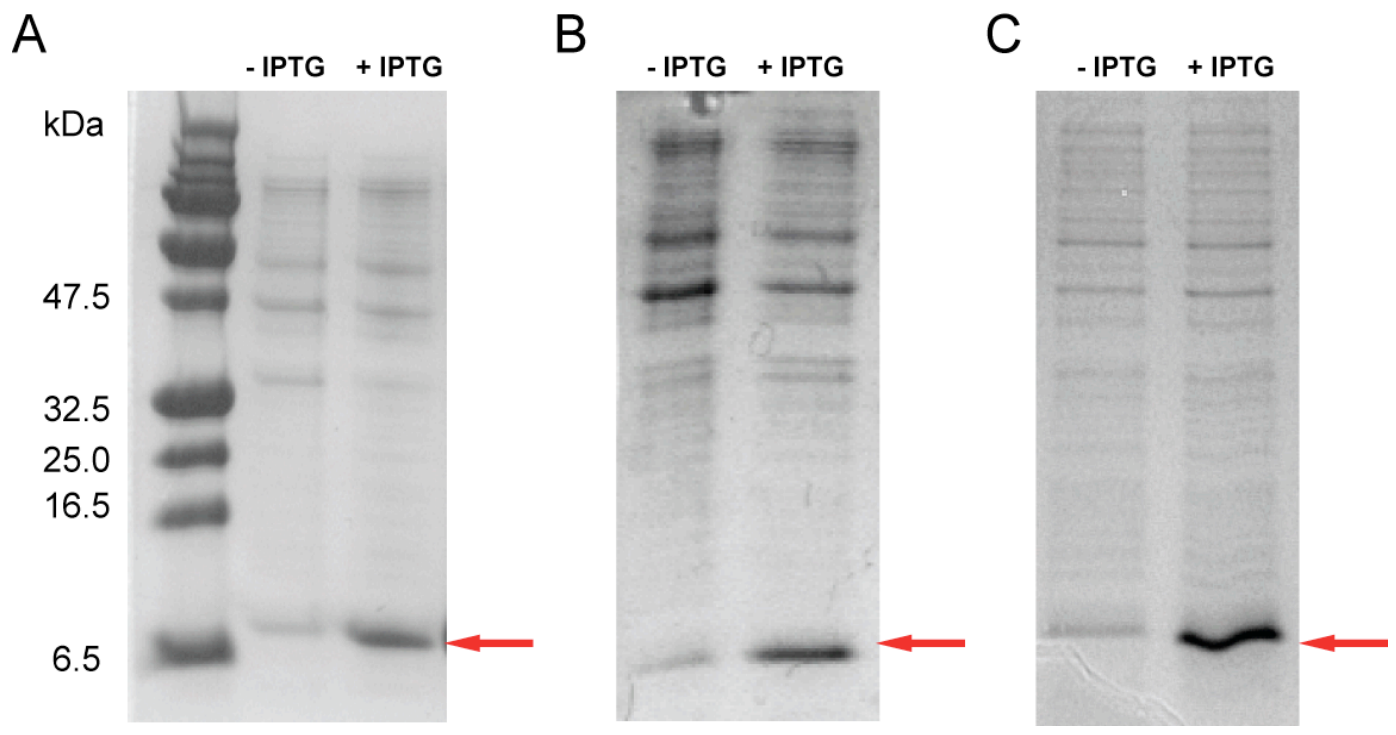

Figure 3.4 Expression of recombinant S100A11 proteins.

Expression of S100A11 (A), S100A11 ${ }^{\mathrm{LII}}$ (B) and S100A11 ${ }^{\mathrm{H} 2 \mathrm{~L}-\mathrm{H} 3}$ (C). Samples of chimaeric S100A11 bacterial cultures before (-IPTG) and following induction with 0.4 mM IPTG at $37{ }^{\circ} \mathrm{C}$ for 4 hours (+IPTG) were loaded onto Tris-Tricine SDS PAGE $15 \%$ gels followed by staining with Coomassie Blue. Strong bands at $\sim 10 \mathrm{kDa}$ (red arrows) show the high levels of expression for chimaeric S100A1 $1^{\mathrm{N} 66 \mathrm{C}}$, E75S $(\mathrm{B})$ and S100A1 $1^{\mathrm{H} 2 \mathrm{~L}-}$ ${ }^{\mathrm{H} 3}$ (C) comparable to that of S100A11 wild-type (A). Molecular weight markers in $\mathrm{kDa}$ on the left of the figure. 
A

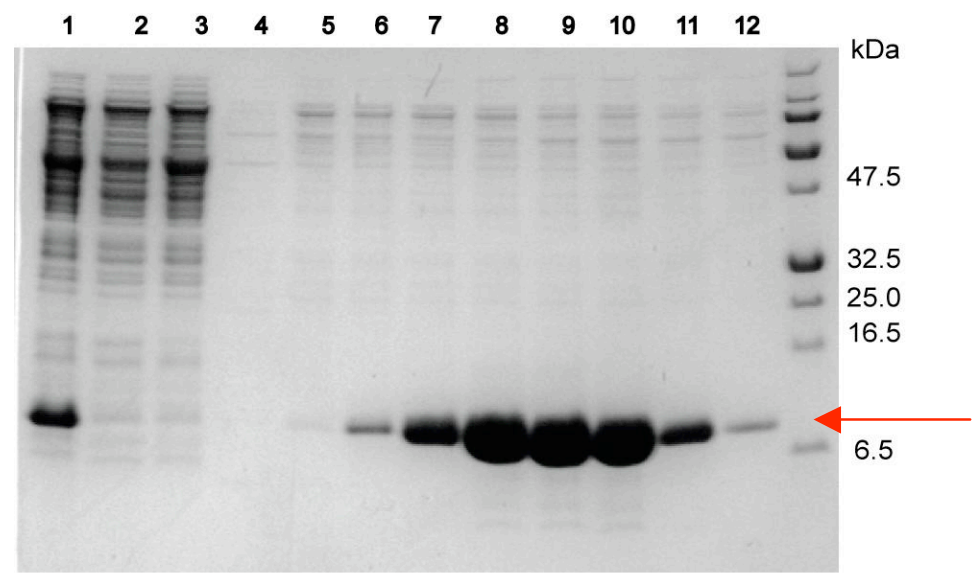

B

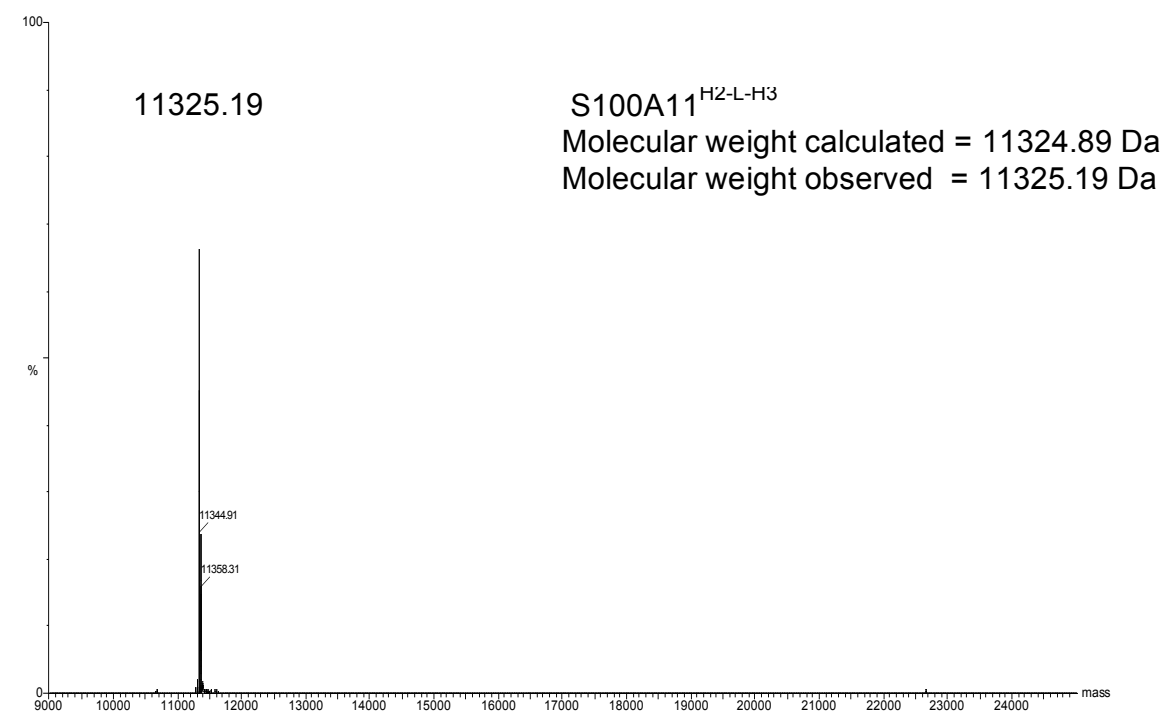

\section{Figure 3.5 Purification of recombinant S100A11 ${ }^{1 \mathrm{H} 2-\mathrm{L}-\mathrm{H} 3}$.}

Purification of S100A $11^{\mathrm{H} 2-\mathrm{L}-\mathrm{H} 3}$ by hydrophobic chromatography monitored by $15 \%$ SDSPAGE stained with Coomassie blue (A). Lane 1, cell lysate prior to purification; lanes 24 flow through material as the phenyl Sepharose column was extensively washed with buffer containing calcium; lanes 5-12, elution fractions after removal of calcium by addition of EGTA buffer. Molecular weight standards are labeled to the right of the figure. Purified S100A11 ${ }^{\mathrm{H} 2-\mathrm{L}-\mathrm{H} 3}$ migrated as a single band $\sim 10 \mathrm{kDa}$ in agreement with monomeric molecular weight of the protein (red arrow). S100A11 ${ }^{\mathrm{H} 2-\mathrm{L}-\mathrm{H} 3}$ was further purified on a size exclusion column and its molecular weight confirmed by electrospray ionization mass spectrometry (B). 
the phenyl Sepharose matrix (see Chapter 4). Efficiency was significantly improved by eluting the protein in a buffer containing EGTA in the absence of salt. After dialyses and lyophilization the average yield of dry weight protein from a one liter culture for the chimaeric proteins purified on a phenyl Sepharose column were as follows: $\sim 100 \mathrm{mg}$ of unlabeled S100A11 and S100A11 ${ }^{\mathrm{LI}} ; 40-50 \mathrm{mg}$ of unlabeled S100A11 ${ }^{\mathrm{H} 2-\mathrm{L}-\mathrm{H} 3}$ and $30 \mathrm{mg}$ for $\mathrm{S} 100 \mathrm{~A} 11^{\mathrm{H} 3, \mathrm{H} 4}$.

Purification of the S100A11 proteins on a phenyl-Sepharose column involves a calcium-induced exposure of hydrophobic residues required to bind the matrix. Chimaeric S100A11 ${ }^{\mathrm{N} 66 \mathrm{C}, \mathrm{E} 75 \mathrm{~S}}, \mathrm{~S} 100 \mathrm{~A} 11^{\mathrm{LII}}, \mathrm{S} 100 \mathrm{~A} 11^{\mathrm{LI}, \mathrm{L} 2}$ contain substitutions in their high affinity calcium-biding loop and are predicted to lack $\mathrm{Ca}^{2+}$-binding ability. Therefore, the development of a novel purification was necessary. Chimaeric proteins with an inactive site II display high pI values from 7.7 for S100A11 ${ }^{\text {N66C, E75S }}$ to 9.1 for S100A11 ${ }^{\text {LII }}$, which allowed their purification using cation exchange chromatography. Cell lysate of S100A11 proteins with modified loop II were loaded into a cationic exchange column and eluted using a linear salt gradient, with each protein exhibiting similar elution profiles. $\mathrm{S} 100 \mathrm{~A} 11^{\mathrm{N} 66 \mathrm{C}, \mathrm{E} 75 \mathrm{~S}}$ eluted at $110 \mathrm{mM} \mathrm{NaCl}$ (Figure 3.6 peak $280 \mathrm{~mL}$ and lanes $7-13$ in $15 \%$ SDS-PAGE gel), while S100A1 $1^{\text {LII }}$ eluted at $130 \mathrm{mM} \mathrm{NaCl}$ and S100A11 ${ }^{\text {LI, LII }}$ eluted at $170 \mathrm{mM} \mathrm{NaCl}$. Ion exchange chromatography produced essentially pure S100A11 ${ }^{\text {N66C, E75S }}$ (Figure 3.6) (similar results were obtained for S100A11 ${ }^{\text {LII }}$ and $\mathrm{S} 100 \mathrm{~A} 11^{\mathrm{LI}, \mathrm{LII}}$ ) as evident by the presence of a single band $\sim 10 \mathrm{kDa}$ in the SDS-PAGE gel for the eluted fractions. Electrospray mass spectrometry was used to verify the mass of the protein. The data in Figure 3.7 shows the presence of two species corresponding to 


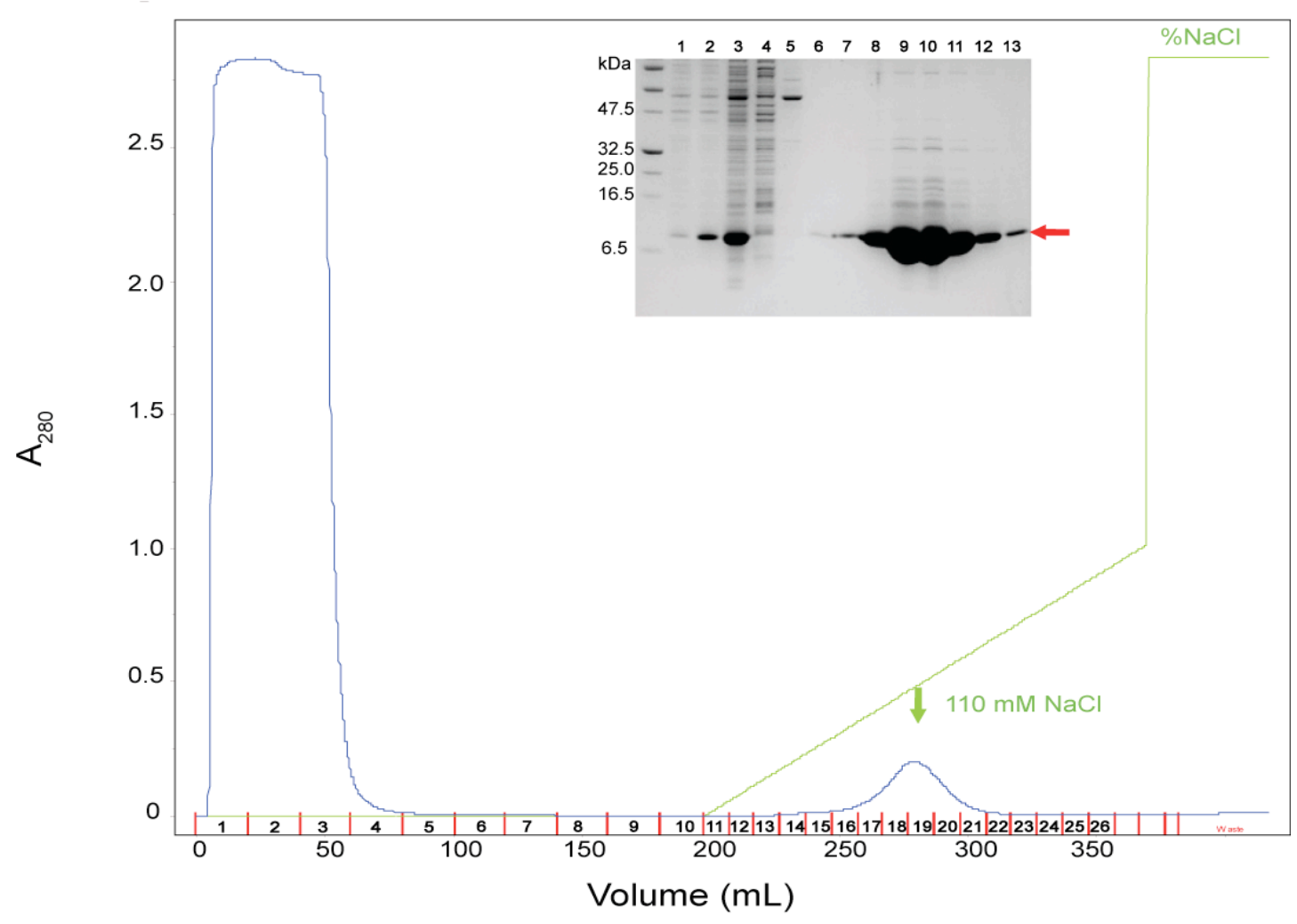

Figure 3.6 Purification of recombinant S100A11 ${ }^{\text {N66C,E75S }}$.

Elution profile of unlabeled S100A11 ${ }^{\mathrm{N} 66 \mathrm{C}, \mathrm{E} 75 \mathrm{~S}}$ using cationic exchange chromatography. Cell lysate dialyzed in $25 \mathrm{mM}$ MES pH 5.8, $1 \mathrm{mM}$ EDTA and $1 \mathrm{mM}$ DTT were applied to a HiTrap SP-XL column (GE Health Care) equilibrated in the same buffer and eluted using a linear $\mathrm{NaCl}$ gradient $(20 \mathrm{mM} \mathrm{NaCl} / \mathrm{min})$. The elution profile shows a wide peak from 0 to $\sim 75 \mathrm{~mL}$ which corresponds to the unbound material that passed through the column and a second peak at $\sim 250 \mathrm{~mL}$ containing purified S100A11 ${ }^{\mathrm{N} 66 \mathrm{C}, \mathrm{E} 75 \mathrm{~S}}$ eluted at 110 $\mathrm{mM} \mathrm{NaCl}$. The inset shows the purification monitored by $15 \%$ SDS-PAGE stained with Coomassie blue. Lane 1, cell extract prior to IPTG induction, Lane 2, cell extract after induction with $0.4 \mathrm{mM} \mathrm{IPTG}$ at $37^{\circ} \mathrm{C}$; lane 3 , crude extract loaded onto the column prior to purification; lane 4-6 flow through fractions (fractions 4, 5 and 7 in chromatogram), lane 7-9 eluted fractions at $110 \mathrm{mM} \mathrm{NaCl}$ corresponding to purified S100A11. 
$\mathrm{S} 100 \mathrm{~A} 11^{\mathrm{N} 66 \mathrm{C}, \mathrm{E} 75 \mathrm{~S}}$ protomer $\left(\mathrm{MW}_{\text {obs }}=11229.04 \mathrm{Da}, \mathrm{MW}_{\text {calc }}=11228.94 \mathrm{Da}\right)$ and the dimeric species $\left(\mathrm{MW}_{\text {obs }}=22456.71, \mathrm{Da} \mathrm{MW}_{\text {calc }}=22457.80 \mathrm{Da}\right)$. The presence of the dimeric species was only seen in the mass spectrometry spectrum for S100A11 proteins with an inactive site II, where a cysteine residue was substituted in the third position of the loop. This cysteine has been shown to easily form disulphide bridges with neighboring molecules in S100A10 alone and in the S100A10-Annexin A2 complex [19], and could be forming a similar disulphide bonds in the chimaeric S100A11 proteins. Average yield of dry weight protein from a one liter culture for the chimaeric proteins purified via ionic exchange chromatography were as follows: $\sim 130 \mathrm{mg}$ of unlabeled $\mathrm{S} 100 \mathrm{~A} 11^{\mathrm{N} 66, \mathrm{E} 75 \mathrm{~S}} ; 80-100 \mathrm{mg}$ of S100A11 ${ }^{\mathrm{LII}}$ and S100A11 ${ }^{\mathrm{LI}, \mathrm{LII}}$.

Purified S100A11 proteins migrated as a single Coomassie blue staining band on SDS-PAGE gels $(15 \%)$ at $\sim 10 \mathrm{kDa}$, in agreement with the molecular weights calculated for the chimaeric proteins (Figure 3.8) from their amino acid sequence. Purified S100A11 proteins were analyzed using denaturing SDS-PAGE with and without addition of reducing agent (DTT) (Figure 3.8). A single band for each S100A11 protein was observed in the presence of DTT, while in the absence of reducing agent the formation of a dimer is evident in all the proteins. Moreover, similar to S100A10, unreduced proteins easily oxidized giving rise to oligomeric forms of the protein [19].

Concluding, all recombinant S100A11 proteins were cloned and expressed as soluble proteins. Purification protocols successfully yielded proteins with high degrees of purity ( $>99 \%$ ), as confirmed by the presence of a single band in SDS-PAGE gels under reducing conditions. Further, the molecular mass and integrity of chimaeric proteins were 
MW calculated dimer $=22457.80 \mathrm{Da}$

MW observed dimer $=22456 . .71 \mathrm{Da}$

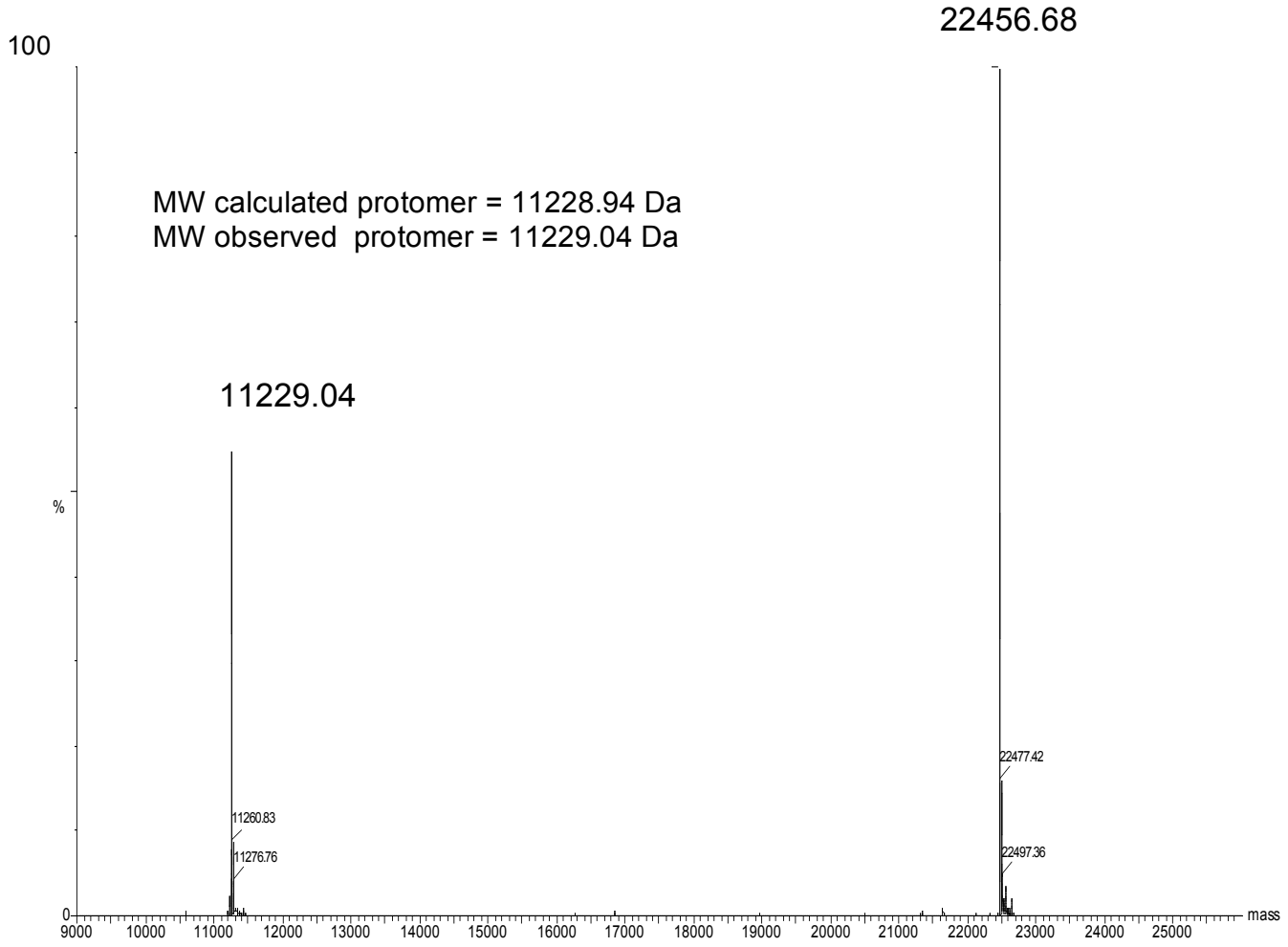

\section{Figure 3.7 Mass Spectrum of S100A11 ${ }^{\text {N66C,E75S }}$.}

Electrospray mass spectrum of purified, freeze dried S100A11 ${ }^{\text {N66C, E75S }}$. Two peaks were observed in the deconvoluted spectrum. Peak A represents the molecular weight (MW) of the species of the subunits of the protein (11229.04 Da) while peak B corresponds to the dimeric species of S100A11 ${ }^{\mathrm{N} 66 \mathrm{C}, \mathrm{E} 75 \mathrm{~S}}(22456.71 \mathrm{Da})$ No evidence of degradation products or impurities are observed in the spectrum indicating the integrity of the purified protein. 
verified using electrospray mass spectrometry (Table 3.2). The molecular masses of all proteins were found in excellent agreement with the calculated from their amino acid sequence.

\subsubsection{Characterization of chimaeric S100A11/S100A10 proteins}

The goal of the chimaeric S100A11/S100A10 design was to understand how different regions of S100A10 might modify the tertiary structure of S100A11. As an initial step, the structural integrity of the chimaeric proteins was evaluated since several of the chimeras included large substitutions that could potentially disrupt their folding.

\subsubsection{Oligomerization states of chimaeric proteins}

In S100 proteins the dimer interface is mediated by helices I and IV of each subunit. Some of the chimaeric proteins contain substitutions in helix IV, which could potentially have effects on the dimer stability. Since dimerization is essential for S100 protein activity, the oligomerization state of all chimaeric proteins was determined by dynamic light scattering (DLS) and sedimentation equilibrium.

DLS is a technique that measures the time dependent fluctuations in the intensities of scattered light. Analysis of these intensities for each chimaeric protein allowed the calculation of diffusion coefficients of the particles, which were converted into size distribution to determine hydrodynamic radius $\left(\mathrm{R}_{\mathrm{H}}\right)$ [21]. $\mathrm{R}_{\mathrm{H}}$ values were fitted to a monomodal curve of molecular weight as a function of $\mathrm{R}_{\mathrm{H}}$ of globular proteins to obtain apparent molecular weights of the proteins. 

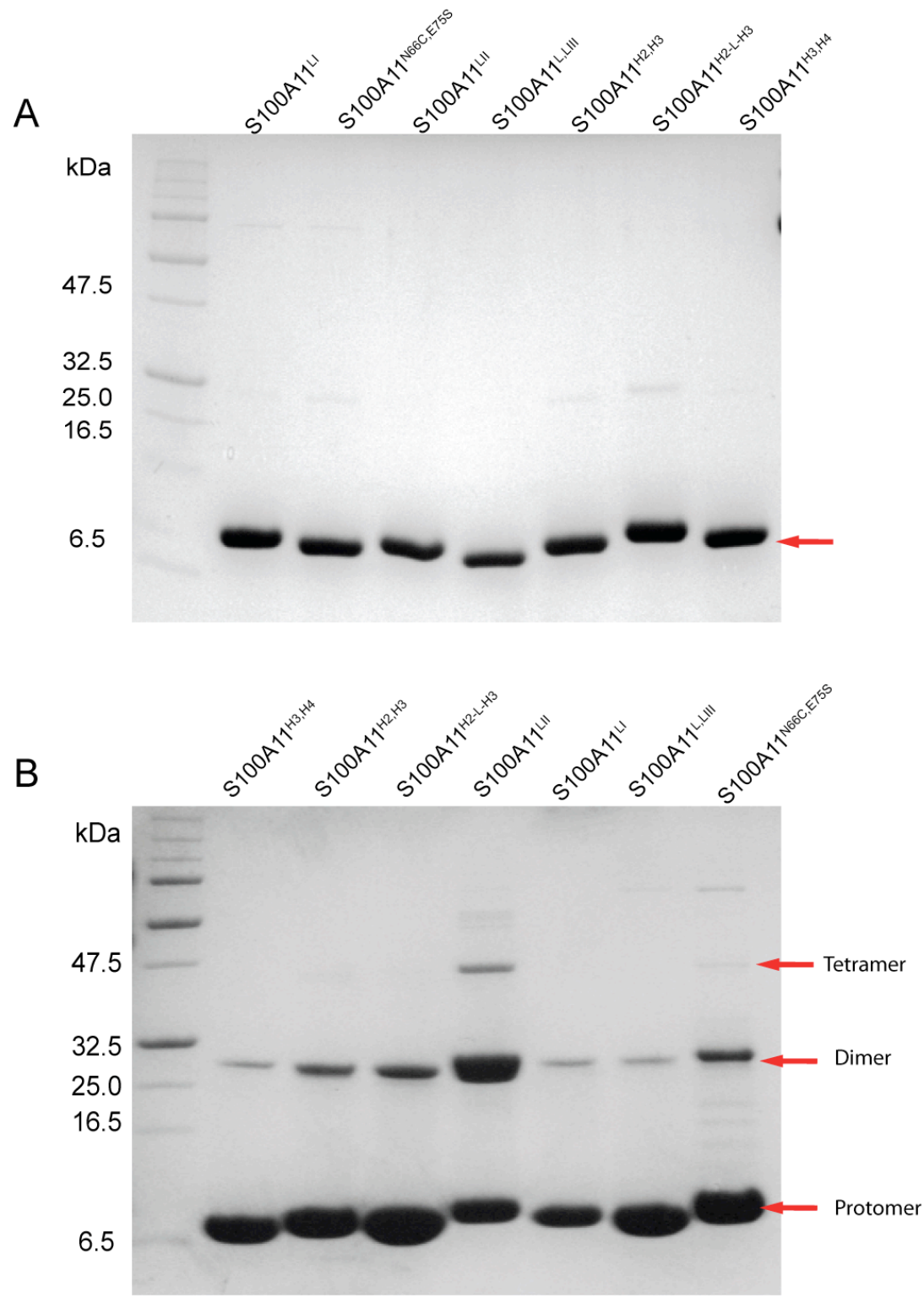

Figure 3.8 Purification of chimaeric S100A11/S100A10 proteins

SDS-PAGE (15\%) analysis of purified recombinant S100A11/S100A10 proteins under reducing (A) and non-reducing conditions (B). All S100A11 proteins migrated with apparent molecular weight $\sim 10 \mathrm{kDa}$. In the presence of fresh DTT (added before loading the samples on the gel) a single band of S100A11 proteins is observed (red arrow in A), but formation of oligomers is observed if proteins with cysteine substitution in site II were not reduced, due to the formation of disulfide bonds between adjacent molecules. Molecular-mass markers are in $\mathrm{kDa}$ on the left of the gel. The gel was stained with Coomasie blue. 
Table 3.2 Mass determination of chimaeric proteins by electrospray mass spectrometry

\begin{tabular}{|c|c|c|c|}
\hline Protein & $\begin{array}{c}\text { Molecular Mass } \\
{[20]} \\
{\text { (Calculated })^{\mathrm{a}}}^{\text {(Calcula }}\end{array}$ & $\begin{array}{c}\text { Molecular Mass } \\
\text { [20] } \\
\text { (Observed) }\end{array}$ & $\begin{array}{c}\text { Difference } \\
{[20]} \\
\text { | Observed-Calculated }\end{array}$ \\
\hline S100A11 & 11281.9 & 11282.1 & 0.2 \\
\hline $\mathrm{S} 100 \mathrm{~A} 11^{\mathrm{LI}}$ & 11048.6 & 11048.3 & 0.3 \\
\hline $\mathrm{S} 100 \mathrm{~A} 11^{\mathrm{N} 66 \mathrm{C}, \mathrm{E} 75 \mathrm{~S}}$ & 11228.9 & 11229.4 & 0.5 \\
\hline $\mathrm{S} 100 \mathrm{~A} 11^{\mathrm{LII}}$ & 11240.9 & 11240.8 & 0.1 \\
\hline $\mathrm{S} 100 \mathrm{~A} 11^{\mathrm{LI}, \mathrm{LII}}$ & 11007.7 & 11008.2 & 0.5 \\
\hline $\mathrm{S} 100 \mathrm{~A} 11^{\mathrm{H} 2, \mathrm{H} 3}$ & 11266.0 & 11266.1 & 0.1 \\
\hline $\mathrm{S} 100 \mathrm{~A} 11^{\mathrm{H} 2-\mathrm{L}-\mathrm{H} 3}$ & 11324.9 & 11325.2 & 0.3 \\
\hline $\mathrm{S} 100 \mathrm{~A} 11^{\mathrm{H} 3, \mathrm{H} 4}$ & 11301.9 & 11302.0 & 0.1 \\
\hline S100A10 & 11614.6 & 11614.2 & 0.4 \\
\hline
\end{tabular}

${ }^{\mathrm{a}}$ The calculated molecular weight (protomer) based on amino acid sequence without initiation methionine.

${ }^{a}$ Only peaks corresponding to protomer species are reported. 
Figure 3.9 shows representative DLS data for S100A11, S100A11 ${ }^{\text {LII }}$ and $\mathrm{S} 100 \mathrm{~A} 11^{\mathrm{H} 3, \mathrm{H} 4}$ proteins at $24{ }^{\circ} \mathrm{C}$. All apo-S100A11 proteins in $25 \mathrm{mM}$ Tris $\mathrm{pH} 7.2,150$ $\mathrm{mM} \mathrm{NaCl}, 1 \mathrm{mM}$ TCEP and $5 \mathrm{mM}$ EDTA showed a narrow monomodal size distribution with slight variation in the width of the peaks and similar $R_{H}$ values (Table 3.3), possibly from a dispersion of particle size in relation to $\mathrm{R}_{\mathrm{H}}$ [22].

The DLS results summarized in Table 3.3 indicated similar $R_{H}$ values for all chimaeric proteins, about 5-7\% smaller than S100A11. Moreover, the radii of hydration obtained for S100A11 and substituted S100A11 proteins are in agreement with previously reported $R_{H}$ values for the dimeric form of S100A11 determined by translational diffusion experiments $\left(\mathrm{R}_{\mathrm{H}}=2.5 \mathrm{~nm}\right.$ dimer; and $\mathrm{R}_{\mathrm{H}}=2.1 \mathrm{~nm}$ protomer $)$ [23] and with the calculated theoretical value from the three-dimensional structure of apoS100A11 [17] using the program Hydropro [24]. According to the DLS analyses described all chimaeric proteins remain as dimers and the substitutions did not disrupt its formation.

Another useful analytical method for studying protein tertiary or quaternary structure in solution is sedimentation equilibrium. It measures the equilibrium concentration distribution in the presence of a centrifugal field. Equilibrium is reached when the effect of centrifugal force on the samples is balanced by diffusion. The distribution is dependent on the molecular weight of the macromolecule and not its shape, unlike DLS that uses a spherical approximation to estimate the hydrodynamic radius. Calcium-free S100A11/S100A10 proteins were studied by sedimentation equilibrium at 

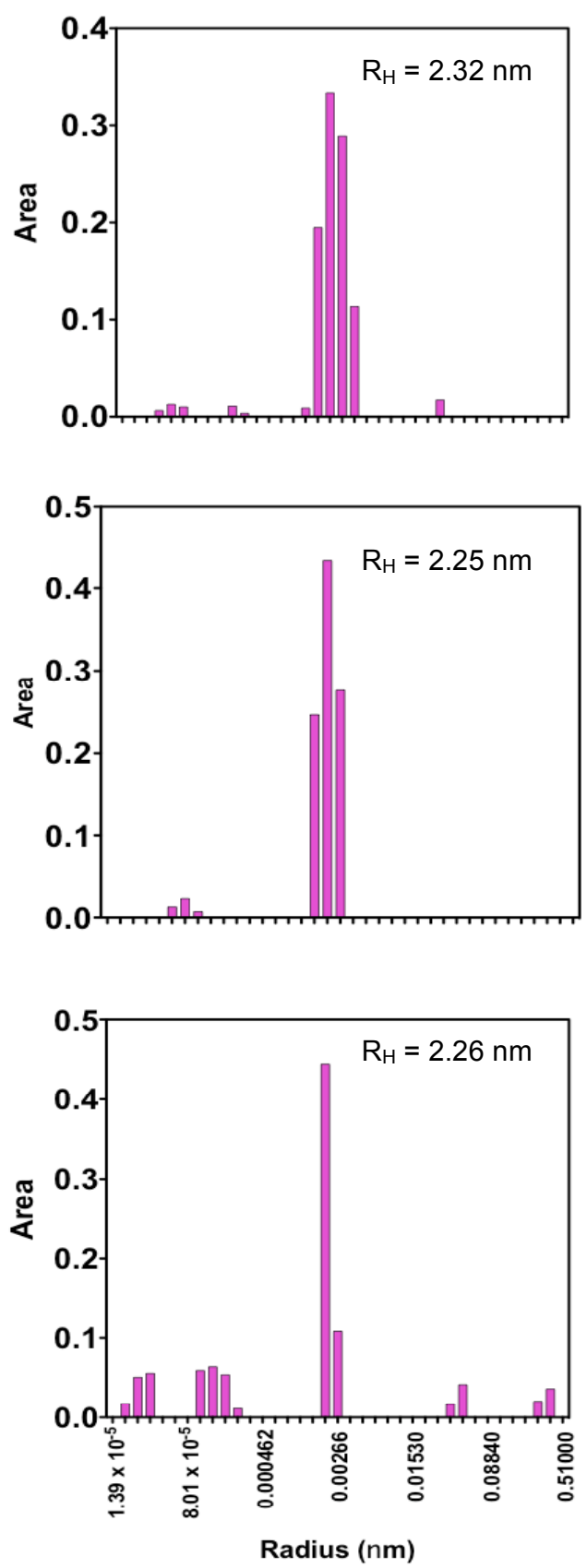

Figure 3.9 Size distribution histogram for S100A11 proteins

Size distribution histogram for S100A11 (top), S100A11 ${ }^{\mathrm{LII}}$ (middle) and S100A11 ${ }^{\mathrm{H} 3, \mathrm{H} 4}$ (bottom) at $24^{\circ} \mathrm{C}$. Chimaeric proteins in the absence of calcium showed monodispersity as illustrated by the presence of only one major region of uniform distribution. 
Table 3.3 Summary of oligomerization analyses for S100A11 proteins

\begin{tabular}{ccccc}
\hline Protein & $\begin{array}{c}\text { Molecular Mass } \\
(\mathbf{k D a}) \\
(\text { Calculated) }\end{array}$ & $\begin{array}{c}\mathbf{R}_{\mathbf{H}}^{\mathbf{b}} \\
(\mathbf{n m})\end{array}$ & $\begin{array}{c}\text { Molecular Mass } \\
(\mathbf{k D a}) \\
\mathbf{D L S}^{\mathbf{c}}\end{array}$ & $\begin{array}{c}\text { Molecular Mass } \\
(\mathbf{k D a}) \\
\text { Sedimentation }^{\mathbf{d}}\end{array}$ \\
\hline $\mathrm{S} 100 \mathrm{~A} 11$ & 22.6 & 2.32 & $24.1 \pm 0.1$ & $25.4 \pm 0.2$ \\
$\mathrm{~S} 100 \mathrm{~A} 11^{\mathrm{LI}}$ & 22.1 & 2.24 & $22.5 \pm 0.2$ & $21.8 \pm 0.2$ \\
$\mathrm{~S} 100 \mathrm{~A} 11^{\mathrm{N} 66 \mathrm{C}, \mathrm{E} 75 \mathrm{~S}}$ & 22.5 & 2.27 & $22.8 \pm 0.2$ & $19.6 \pm 0.1$ \\
$\mathrm{~S} 100 \mathrm{~A} 11^{\mathrm{LII}}$ & 22.5 & 2.25 & $22.6 \pm 0.2$ & $20.6 \pm 0.1$ \\
$\mathrm{~S} 100 \mathrm{~A} 11^{\mathrm{LI}, \mathrm{LII}}$ & 22.0 & 2.24 & $22.5 \pm 0.3$ & $20.3 \pm 0.1$ \\
$\mathrm{~S} 100 \mathrm{~A} 11^{\mathrm{H} 2, \mathrm{H} 3}$ & 22.5 & 2.21 & $21.7 \pm 0.2$ & $23.4 \pm 0.2$ \\
$\mathrm{~S} 100 \mathrm{~A} 11^{\mathrm{H} 2-\mathrm{L}-\mathrm{H} 3}$ & 22.5 & 2.17 & $21.6 \pm 0.2$ & $24.0 \pm 0.2$ \\
$\mathrm{~S}_{100 \mathrm{~A} 11^{\mathrm{H} 3, \mathrm{H} 4}}$ & 22.6 & 2.26 & $22.8 \pm 0.4$ & $19.9 \pm 0.1$ \\
\hline
\end{tabular}

${ }^{a}$ Molecular weight calculated based on the nucleotide sequence for dimeric form of the protein

${ }^{\mathrm{b}} \mathrm{R}_{\mathrm{H}}=$ Hydrodynamic radius determined by DLS

${ }^{c}$ Molecular mass determined from DLS using a monomodal curve fit of molecular mass as a function of $\mathrm{R}_{\mathrm{H}}$ of globular proteins (DYNAMICS software). Average of triplicate readings

${ }^{\mathrm{d}}$ Molecular mass determined from sedimentation equilibrium experiments using a global fit for six data sets at 25000 and $30000 \mathrm{rpm}$. All data sets were globally fitted to a single species model (GraphPad Prism 4.0) 
rotor speeds of 25000 to $30000 \mathrm{rpm}$ at $24{ }^{\circ} \mathrm{C}$. Figure 3.10 shows the equilibrium curves or apo-S100A11 fitted to a singles species model.

The calculated residuals are randomly distributed, indicating that the single ideal specials mode used for the fit is appropriate. Further, each chimaeric S100A11/S100A10 was subjected to sedimentation analyses as summarized in Table 3.3 and depicted in Figure 3.11 for S100A $11^{\mathrm{N} 66 \mathrm{C}, \mathrm{E} 75 \mathrm{~S}}(\mathrm{~A})$ and $\mathrm{S} 100 \mathrm{~A} 11^{\mathrm{H} 3, \mathrm{H} 4}(\mathrm{~B})$. In all cases data sets were fit individually and globally to a single species model. Apparent molecular weight was close to the calculated mass of the dimer form of the S100A11 proteins.

In summary, dynamic light scattering and sedimentation equilibrium experiments showed the molecular masses for all chimaeric proteins correspond to the dimer form of S100A11. Moreover neither high order oligomers nor evidence of disruption of the dimers was evident.

\subsubsection{Folded state of S100A11/S100A10 proteins}

\subsection{Apo-S100A11 proteins retain $\alpha$-helical structure}

Circular dichroism (CD) spectropolarimetry was used to probe the proper folding and secondary structure of apo-S100A11, $\mathrm{Ca}^{2+}-\mathrm{S} 100 \mathrm{~A} 11, \mathrm{~S} 100 \mathrm{~A} 10$ and the seven chimaeric proteins. As shown in Figure 3.12, all proteins displayed characteristic $\alpha$ helical spectra with two minima near $208 \mathrm{~nm}$ and $222 \mathrm{~nm}$, indicating that substitutions did not disrupt the overall helical structure; even when large numbers of substitutions were displayed a spectrum of similar magnitude to apo-S100A11 but with altered $\theta_{222} / \theta_{208}=1.05$ indicative of different interhelical interactions within these two proteins. 

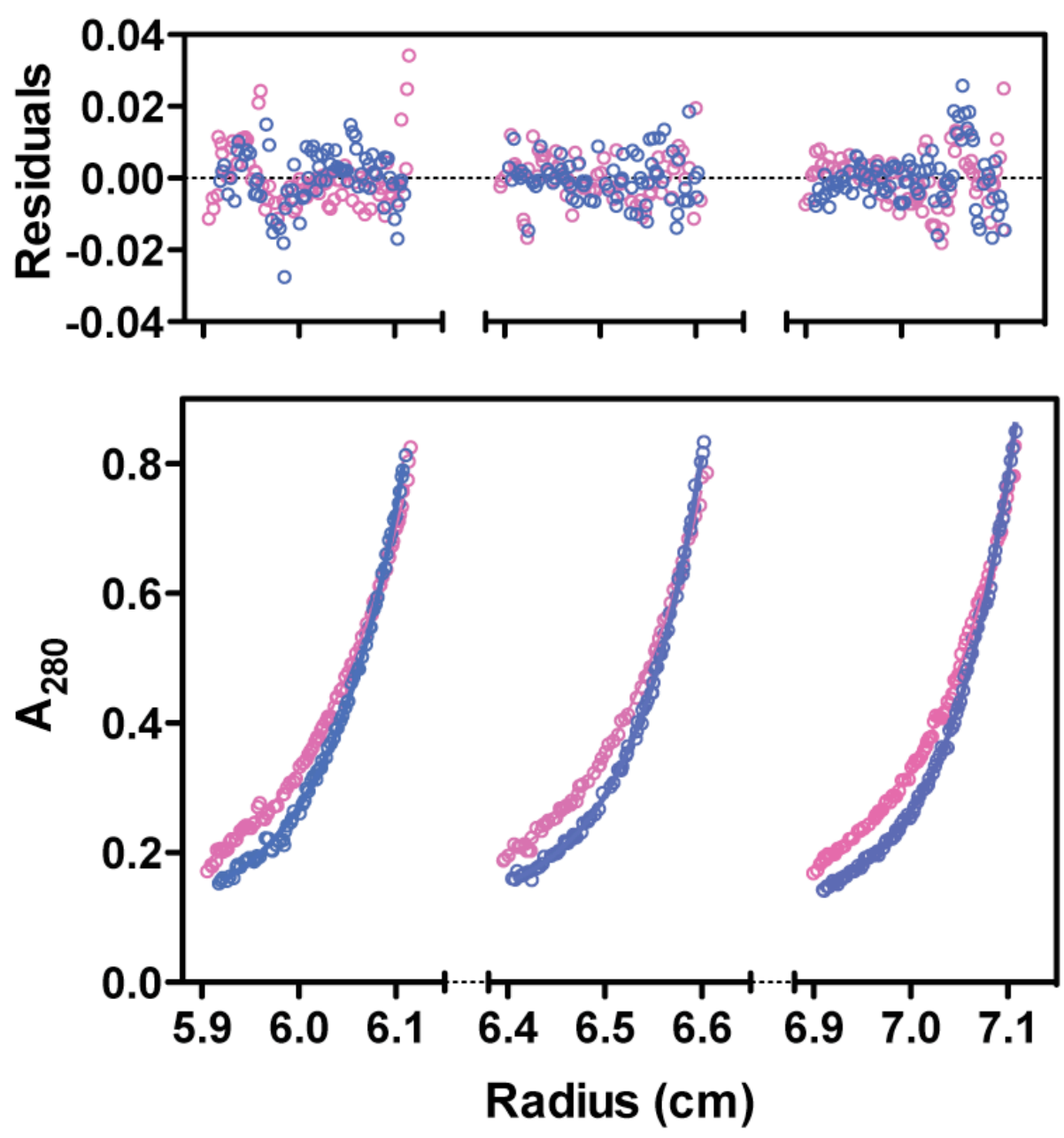

Figure 3.10 Sedimentation equilibrium curves for apo-S100A11

Representative equilibrium data set for a solution of $45 \mu \mathrm{M} \mathrm{S100A11} \mathrm{in} 20 \mathrm{mM}$ Tris $\mathrm{pH}$ 7.2, $150 \mathrm{mM} \mathrm{NaCl}, 1 \mathrm{mM}$ TCEP and 5mM EDTA at $24{ }^{\circ} \mathrm{C}$. The rotor speeds were 25000 rpm (pink curve) and $30000 \mathrm{rpm}$ (blue curve). Cells were scanned at $280 \mathrm{~nm}$ after equilibrium was reached (16h). Data was globally fit to a single ideal species model, obtaining an average molecular weight of $25.4 \mathrm{kDa}$. Calculated residuals are shown above. 

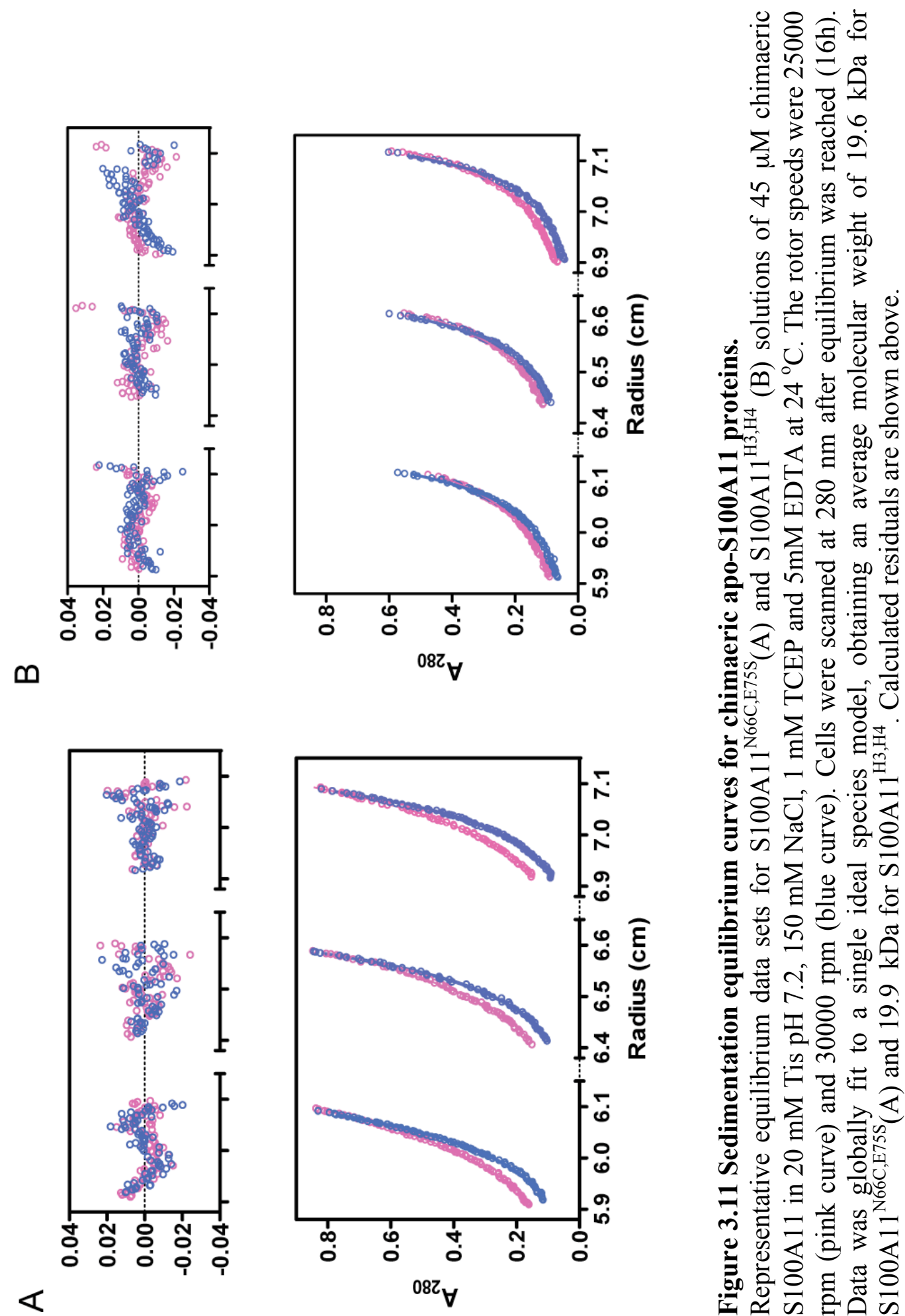

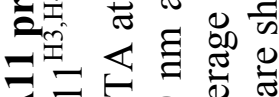

\&

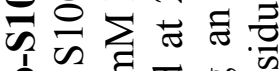

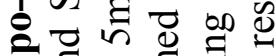

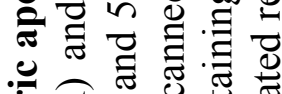
• 焉望

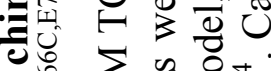

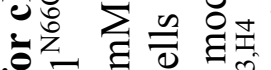
긍 \& 8 نं

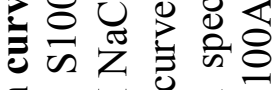

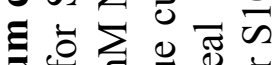

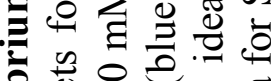
흄

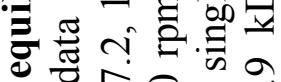
홍 ๘

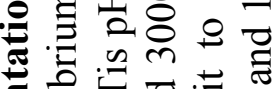

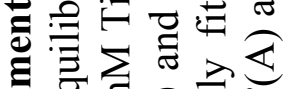

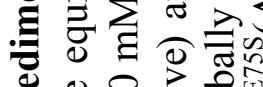

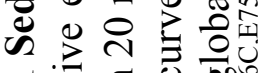

二 昰 ஸे 䒕二 ¿

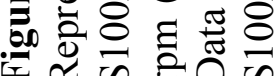


A comparison of the chimera proteins containing substitutions in the calcium-binding sites (Figure 3.12 B) showed a maximum 13\% deviation between the ellipticity at $208 \mathrm{~nm}$ for introduced (18 substitutions in S100A11 $\left.{ }^{\mathrm{H} 2-\mathrm{L}-\mathrm{H} 3}\right)$. The CD spectrum of apo-S100A11 had $\theta_{222} / \theta_{208}=0.93$, similar to that reported for apo-S100B $(0.95)$ [25].

Upon calcium binding the spectrum of S100A11 decreased in magnitude and had a modified $\theta_{222} / \theta_{208}$ ratio of 0.87 . This spectrum is different from that of S100A10 which S100A11 $1^{\text {LI,LII }}$ and apo-S100A11 suggesting that only minor differences between the lengths and/or interactions between the helices existed. For the chimaeric proteins that had modifications in the helices (Figure 3.12 C) the difference in the CD spectra between the individual substituted proteins was less than $1 \%$. These results indicated that all chimaeric proteins studied maintained the same high degree of $\alpha$-helical structure, as seen for S100A11 and S100A10.

\subsection{Apo-S100A11 proteins demonstrate stable tertiary structures}

NMR spectroscopy was used to verify that all proteins were properly folded. Figure 3.13 illustrates the ${ }^{1} \mathrm{H}_{-}{ }^{15} \mathrm{~N}$ HSQC spectrum of apo-S100A11 compared with those for chimaeric proteins having minimal substitutions in the calcium-binding loop of site II $\left(\mathrm{S} 100 \mathrm{~A} 11^{\mathrm{N} 66 \mathrm{C}, \mathrm{E} 75 \mathrm{~S}}\right)$ or a large number of changes in the helices affecting both sites $\left(\mathrm{S} 100 \mathrm{~A} 11^{\mathrm{H} 2-\mathrm{L}-\mathrm{H} 3}\right)$. In the absence of calcium, all chimaeric proteins showed welldispersed spectra with resonances of similar linewidth to apo-S100A11. In addition, assignment of the spectra revealed that no obvious line-broadening or doubling of peaks were evident in the data suggestive of multiple conformations. These observations indicate that all the chimeric S100A11 proteins adopted $\alpha$-helical, dimeric species 

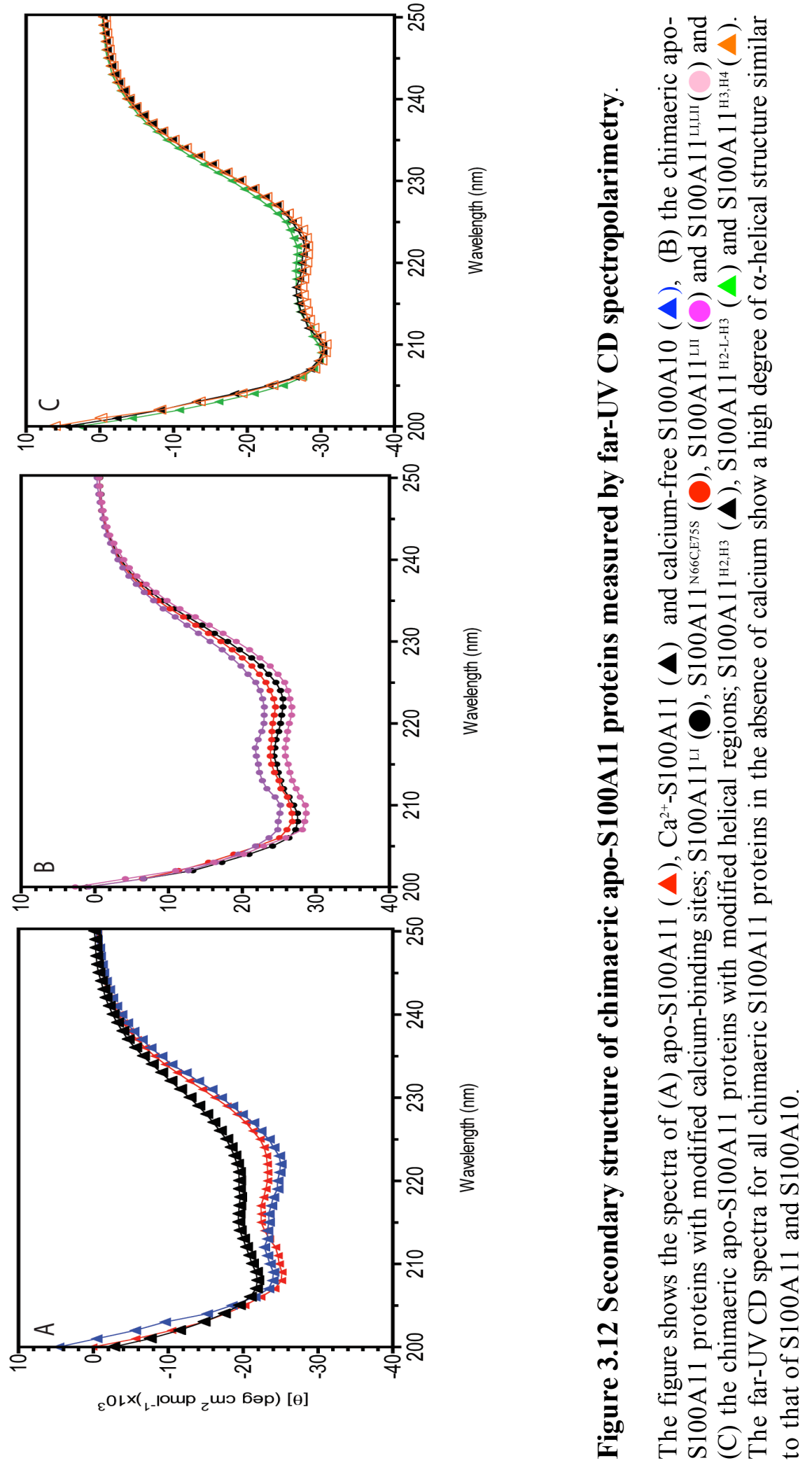

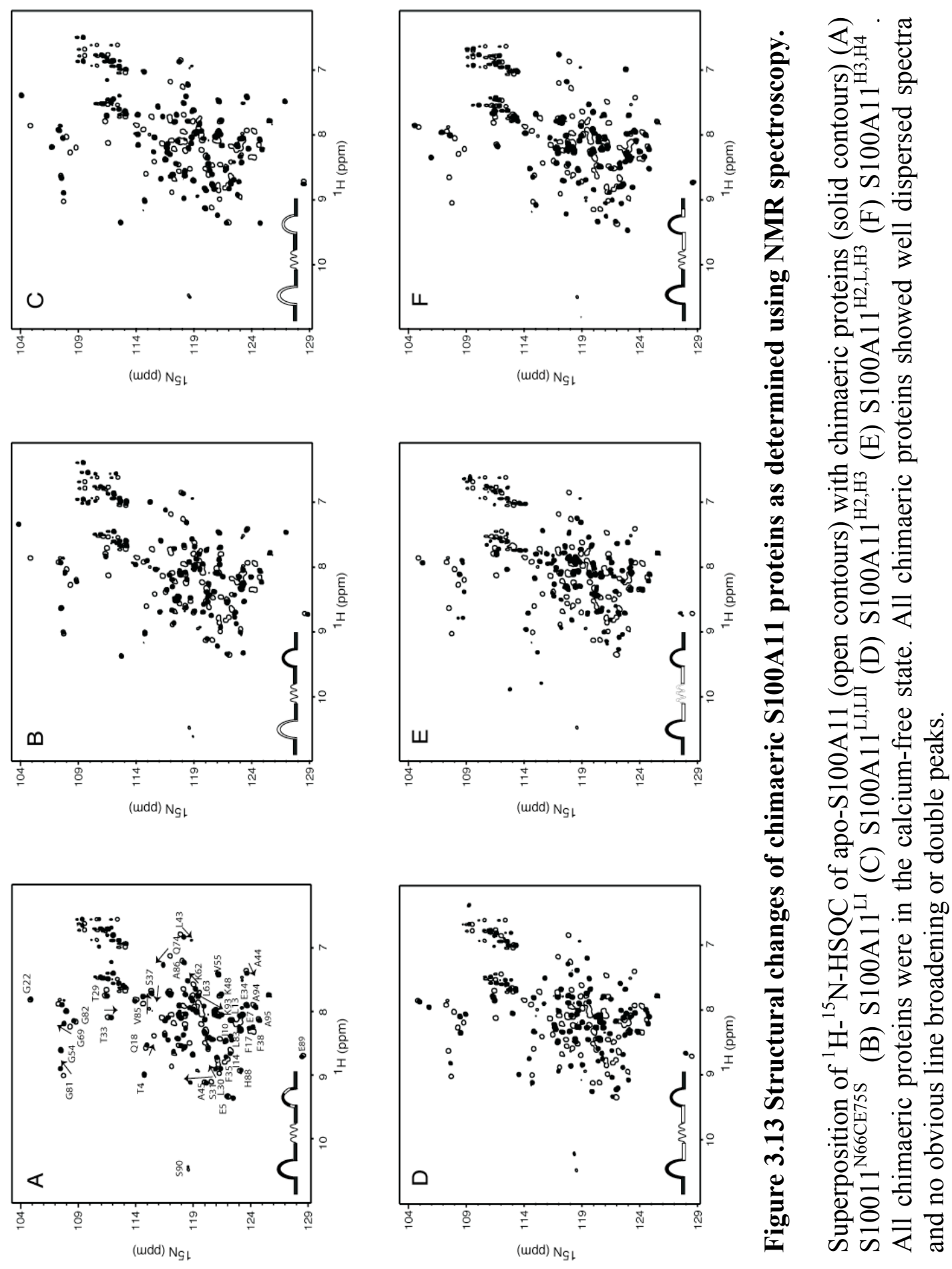
reminiscent of ${ }^{1} \mathrm{H}^{-15} \mathrm{~N}-\mathrm{HSQC}$ spectra of other $\mathrm{S} 100$ proteins.

The observed chemical shift differences between different chimaeric S100A11 proteins and apo-S100A11 were used to pinpoint whether the substitutions simply affected the environment of residues within close proximity to the substitution points or had a more global effect on the structures. For example, Figure 3.13A shows a superposition of the ${ }^{1} \mathrm{H}_{-}{ }^{15} \mathrm{~N}-\mathrm{HSQC}$ spectra for apo-S100A11 and S100A11 ${ }^{\mathrm{N} 66 \mathrm{C}, \mathrm{E} 75 \mathrm{~S}}$. In this data, most of the peaks in helices I (T4, E5, E7, R8, I10, L13), III (G54,V55), Cterminus of helix IV (E89, A94, A95) and portions of calcium-binding site I (G22, G25) for S100A11 ${ }^{\mathrm{N} 66 \mathrm{C}, \mathrm{E} 75 \mathrm{~S}}$ remained in similar positions compared with apo-S100A11 indicating the two-residue substitution had little influence on the environments of these residues. In addition, most of the residues in calcium-binding site II (K62, L63, G69, Q74) which are close in sequence and space $(<6 \AA)$ to the substitution site and several residues near the C-terminus of site I (N-terminus of helix II) including T29, S31, T33 and F35 undergo expected shifts due to proximity to the substitutions. Surprisingly, chemical shift changes were noted in $\mathrm{S} 100 \mathrm{~A} 11^{\mathrm{N} 66 \mathrm{C}, \mathrm{E} 75 \mathrm{~S}}$ for residues in the linker between helices II and III (L43, A44, A45, K48) and in the central portion of helix IV (G81, L83, V85, A86). These two portions are in close contact in the apo-S100A11 structure, yet remote from the sites of substitution, indicating that replacement of residues N66 and E75 in apo-S100A11 had affected the interaction of these two regions. Unlike the S100A11 ${ }^{\text {N66C,E75S }}$ chimera nearly all residues shift in S100A11 ${ }^{\mathrm{H} 2 \mathrm{~L}-\mathrm{H} 3}$ compared to apoS100A11. Since the majority of the replacements helices II, III and the intervening linker have contacts $(<6 \AA)$ with residues outsides these regions this is not surprising. However 
the large number of chemical shift changes made it difficult to discern between local environment changes due to the substitutions and alterations in the protein conformation.

\subsubsection{Most chimaeric $\mathrm{Ca}^{2+}-$ S100A11 proteins can adopt an open conformation}

Binding of calcium to apo-S100A11 leads to a large conformational change exposing previously buried residues. This calcium-induced rearrangement is easily monitored by NMR spectroscopy as the chemical shifts of many residues generate a characteristic ${ }^{1} \mathrm{H}-{ }^{15} \mathrm{~N}-\mathrm{HSQC}$ spectrum significantly different from that of apo-S100A11 (Figure 3.14A). Using NMR spectroscopy the backbone amide correlations were compared for the S100A11 chimaeric proteins in their apo- and calcium-bound forms to determine the ability of these proteins to bind calcium and adopt a more open conformation such as that observed for $\mathrm{Ca}^{2+}-\mathrm{S} 100 \mathrm{~A} 11$.

Substitutions in the second calcium-binding site of S100A11 (S100A11 ${ }^{\text {N66C,E75S }}$ and S100A11 ${ }^{\mathrm{LII}}$ ) (Figure $3.15 \mathrm{~A}, \mathrm{~B}$ ) showed few chemical shift changes upon calcium addition, showing that coordination of calcium to site II was abolished. In particular there was little change in the position of G69 in site II which typically shifts to $\sim 10.5 \mathrm{ppm}$ upon calcium binding to S100A11 (Figure 3.14). There were some minor chemical shift changes observed $(\mathrm{G} 22)$ in S100A11 ${ }^{\mathrm{N} 66 \mathrm{C}, \mathrm{E} 75 \mathrm{~S}}$ suggesting there is weak calcium binding to site I. The chimaeric protein S100A11 ${ }^{\mathrm{LI}, \mathrm{LII}}$ where both calcium-binding loops were replaced with those in S100A10 showed near identical ${ }^{1} \mathrm{H}_{-}{ }^{15} \mathrm{~N}$ HSQC spectra in the absence and presence of calcium indicating that this protein has lost its ability to chelate calcium (Figure 3.15D). Substitutions to the low affinity calcium-binding site alone $\left(\mathrm{S} 100 \mathrm{~A} 11^{\mathrm{LI}}\right)$ (Figure $\left.3.15 \mathrm{C}\right)$, appeared to affect calcium binding to the higher affinity 

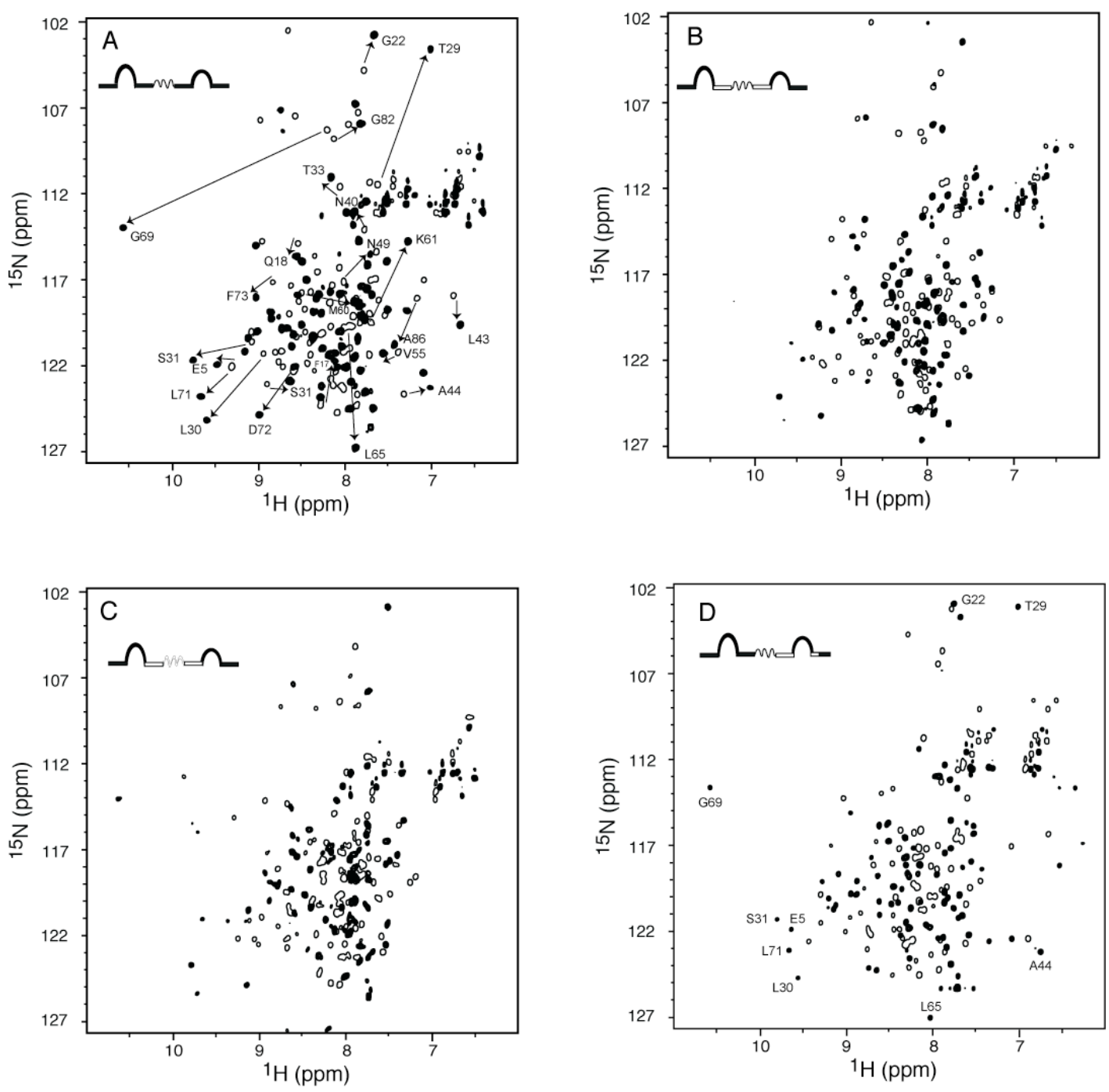

Figure 3.14 Calcium-induced conformational changes in chimaeric proteins with modified helices.

Comparison of ${ }^{1} \mathrm{H}^{15} \mathrm{~N}-\mathrm{HSQC}$ spectra for (A) S100A11, (B) $\mathrm{S} 100 \mathrm{~A} 11^{\mathrm{H} 2, \mathrm{H} 3}$, (C) $\mathrm{S} 100 \mathrm{~A} 11^{\mathrm{H} 2-\mathrm{L}-\mathrm{H} 3}$ and (D) $\mathrm{S} 100 \mathrm{~A} 11^{\mathrm{H} 3, \mathrm{H} 4}$ in the absence (open contours) and presence of calcium (filled contours). Residues that undergo chemical shift changes upon binding of calcium are labeled and arrows are used to show the direction of the chemical shift change. 

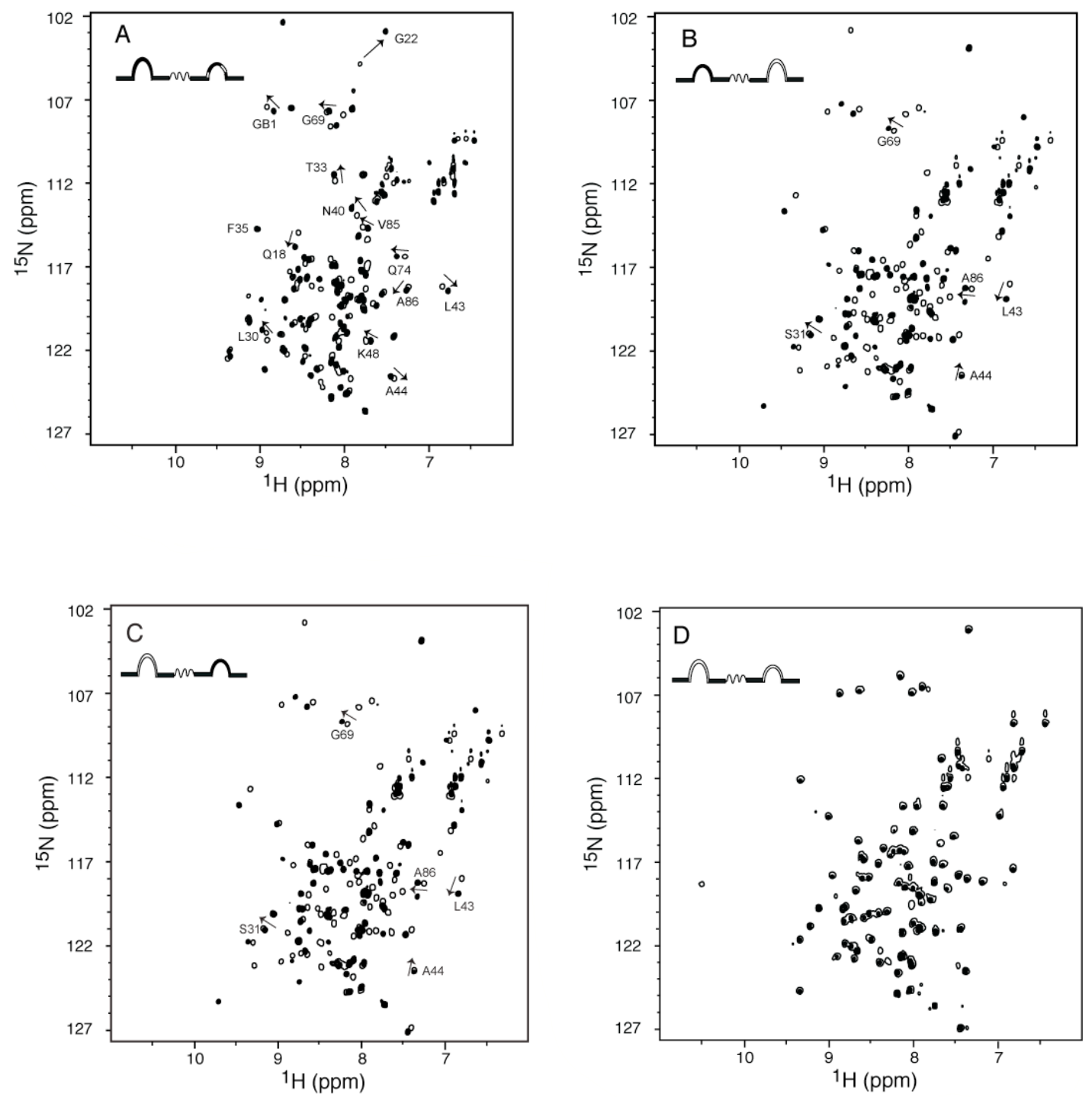

Figure 3.15 Calcium-induced conformational changes in chimaeric proteins with substituted calcium binding loops.

Comparison of ${ }^{1} \mathrm{H}^{15} \mathrm{~N}-\mathrm{HSQC}$ spectra for (A) S100A11 ${ }^{\mathrm{N} 66 \mathrm{C}, \mathrm{E} 75 \mathrm{~S}}$, (B) S100A11 ${ }^{\mathrm{LII}}$, (C) $\mathrm{S} 100 \mathrm{~A} 11^{\mathrm{L} 1}$ and (D) S100A $11^{\mathrm{L} \text {, LII }}$ in the absence (open contours) and presence of calcium (filled contours). Residues that undergo chemical shift changes upon binding of calcium are labeled and arrows are used to show the direction of the chemical shift change. 
calcium-binding sites I or II (S100A11 $\left.{ }^{\mathrm{LI}}, \mathrm{S} 100 \mathrm{~A} 11^{\mathrm{LI}, \mathrm{LI}}, \mathrm{S} 100 \mathrm{~A} 11^{\mathrm{LII}}\right)$ had strongly attenuated calcium-binding ability much like S100A10.

Addition of calcium to the chimaeric proteins having native calcium-binding loops but altered helices $\left(\mathrm{S} 100 \mathrm{~A} 11^{\mathrm{H} 2 \mathrm{H} 3}, \mathrm{~S} 100 \mathrm{~A} 11^{\mathrm{H} 2-\mathrm{L}-\mathrm{H} 3}\right.$ and $\left.\mathrm{S} 100 \mathrm{~A} 11^{\mathrm{H} 3, \mathrm{H} 4}\right)$ resulted in ${ }^{1} \mathrm{H}-{ }^{15} \mathrm{~N}$ HSQC spectra that are typical of $\mathrm{Ca}^{2+}-\mathrm{S} 100 \mathrm{~A} 11$ (Figure 3.14). For example calcium binding to $\mathrm{S} 100 \mathrm{~A} 11^{\mathrm{H} 3, \mathrm{H} 4}$ resulted in large chemical shift changes for residues in both sites I (G22, T29, L30, S31) and II (L65, L71) including the characteristic change in G69. In addition, chemical shift changes were noted for residues L43, A44 in the linker, N49, V55 and M60 in helix III, and A86 and H88 in helix IV that are diagnostic of the large conformational change observed upon calcium binding to S100A11. These results show that large sequence changes in the helices of S100A11 do not prevent it from binding calcium nor from adopting the more open form of the protein required for target binding.

\subsection{Conclusion}

S100A10 was used as a template to design a set of seven chimaeric S100A11 proteins based on the regions where the largest dissimilarities in sequence and structure were found (calcium binding loops and helix III) (Chapter 2). Chimaeric S100A11/S100A10 proteins were designed to examine the influence of either calciumbinding sites or interhelical regions on the overall conformation of S100A11. Substitutions ranged in complexity from two residues in the second calcium-binding loop $\left(\mathrm{S} 100 \mathrm{~A} 11^{\mathrm{N} 66 \mathrm{CE} 75 \mathrm{~S}}\right)$ to the complete replacement of helices II, III and their intervening linker in S100A11 ${ }^{\mathrm{H} 2-\mathrm{L}-\mathrm{H} 3}$ (18 substitutions). This long-range substitution approach 
provides a broad yet extremely efficient method to initially identify the regions that contribute to the permanently active form of S100A10. Fine tuning of the substitutions designed will be performed based on the characterization of the proteins to isolate only essential residues that drive the stabilization of the open form of S100A10 in a calciumindependent manner.

Genes encoding rabbit S100A11 with the desired substitutions were successfully synthesized and expressed in Escherichia coli BL21(D3) strain. Further, the purification protocols developed for the chimaeric S100A11/S100A10 proteins yielded highly pure proteins $(>99 \%)$, with no evidence of degradation products (for unlabeled proteins average yield was $100 \mathrm{mg}$ of weight dried protein from 1L of LB bacterial growth).

Characterization of the engineered S100A11 proteins showed that despite the large number of substitutions made in several of the chimaeric S100A11/S100A10 (18 residues in $\mathrm{S} 100 \mathrm{~A} 11^{\mathrm{H} 2-\mathrm{LH} 3}$ ), all the proteins tested retained their dimeric, $\alpha$-helical structure as evident by dynamic light scattering, sedimentation equilibrium, and circular dichroism spectropolarimetry. Moreover, NMR spectroscopy showed that all chimaeric proteins were properly folded and that substitutions in helical regions in S100A11 do not affect the ability to reach the open conformation in the presence of calcium.

The next step of this research project is to evaluate the structural effects of each substituted region in apo-S100A11. The following chapter (Chapter 4) describes the biophysical and structural approach used. 


\subsection{References}

1 Wang, W. and Malcolm, B. A. (1999) Two-stage PCR protocol allowing introduction of multiple mutations, deletions and insertions using QuikChange Site-Directed Mutagenesis. Biotechniques. 26, 680-682

2 Rety, S., Osterloh, D., Arie, J. P., Tabaries, S., Seeman, J., Russo-Marie, F., Gerke, V. and Lewit-Bentley, A. (2000) Structural basis of the $\mathrm{Ca}(2+)$-dependent association between S100C (S100A11) and its target, the N-terminal part of annexin I. Structure. 8, $175-184$

3 Rintala-Dempsey, A. C., Santamaria-Kisiel, L., Liao, Y., Lajoie, G. and Shaw, G. S. (2006) Insights into S100 target specificity examined by a new interaction between S100A11 and annexin A2. Biochemistry. 45, 14695-14705

4 Sambrook, J. a. R. D. W. (2001) Molecular Cloning. Cold Spring Harbor Laboratory Press, NY, USA

5 Dulau, L., Cheyrou, A., Dubourdieu, D. and Aigle, M. (1989) Directed mutagenesis using PCR. Nucleic Acids Res. 17, 2873

6 Rintala, A. C., Schonekess, B. O., Walsh, M. P. and Shaw, G. S. (2002) 1H, 15N and 13C resonance assignments of rabbit apo-S100A11. J Biomol NMR. 22, 191-192

7 Squire, P. G. and Himmel, M. E. (1979) Hydrodynamics and protein hydration. Arch Biochem Biophys. 196, 165-177

8 Murphy, R. M. (1997) Static and dynamic light scattering of biological macromolecules: what can we learn? Curr Opin Biotechnol. 8, 25-30

9 Kay, L. E., Keifer, P. and Saarinen, T. (1992) Pure absorption gradient enhanced heteronuclear single quantum correlation spectroscopy with improved sensitivity. J. Am. Chem. Soc. 114, 10663-10665

10 Delaglio, F., Grzesiek, S., Vuister, G. W., Zhu, G., Pfeifer, J. and Bax, A. (1995) NMRPipe: A multidimensional spectral processing system based on UNIX pipes. J. Biomol. NMR. 6, 277-293

11 Johnson, B. A. and Belvins, R. A. (1994) NMRView: A computer program for the visualization and analysis of NMR data. J. Biomol. NMR. 4, 603-614

12 Durussel, I., Van Eldik, L. J. and Cox, J. A. (1997) Ion-binding properties of recombinant $\mathrm{S} 100$ beta and two derivatives with either an inactivated $\mathrm{Ca} 2+$ site II or a normalized Ca2+ site I. Biochim Biophys Acta. 1343, 139-143

13 Franz, C., Durussel, I., Cox, J. A., Schafer, B. W. and Heizmann, C. W. (1998) Binding of $\mathrm{Ca} 2+$ and $\mathrm{Zn} 2+$ to human nuclear S100A2 and mutant proteins. J Biol Chem. 273, 18826-18834

14 Grabarek, Z. (2006) Structural basis for diversity of the EF-hand calcium-binding proteins. J Mol Biol. 359, 509-525

15 Bunick, C. G., Nelson, M. R., Mangahas, S., Hunter, M. J., Sheehan, J. H., Mizoue, L. S., Bunick, G. J. and Chazin, W. J. (2004) Designing sequence to control protein function in an EF-hand protein. J Am Chem Soc. 126, 5990-5998

16 Nelson, M. R., Thulin, E., Fagan, P. A., Forsen, S. and Chazin, W. J. (2002) The EFhand domain: a globally cooperative structural unit. Protein Sci. 11, 198-205 
17 Dempsey, A. C., Walsh, M. P. and Shaw, G. S. (2003) Unmasking the annexin I interaction from the structure of Apo-S100A11. Structure (Camb). 11, 887-897

18 Ababou, A. and Desjarlais, J. R. (2001) Solvation energetics and conformational change in EF-hand proteins. Protein Sci. 10, 301-312

19 Rety, S., Sopkova, J., Renouard, M., Osterloh, D., Gerke, V., Tabaries, S., RussoMarie, F. and Lewit-Bentley, A. (1999) The crystal structure of a complex of p11 with the annexin II N-terminal peptide. Nat Struct Biol. 6, 89-95

20 Potts, B. C., Carlstrom, G., Okazaki, K., Hidaka, H. and Chazin, W. J. (1996) 1H NMR assignments of apo calcyclin and comparative structural analysis with calbindin D9k and S100 beta. Protein Sci. 5, 2162-2174

21 Berne, J., and Pecora, R. . (2000) Dynamic Light Scattering with Applications to Chemistry, Biology, and Physics; . Dover Publications: Mineola, NY

22 Papish, A. L., Tari, L. W. and Vogel, H. J. (2002) Dynamic light scattering study of calmodulin-target peptide complexes. Biophys J. 83, 1455-1464

23 Marlatt, N. M., Boys, B. L., Konermann, L. and Shaw, G. S. (2009) Formation of monomeric S100B and S100A11 proteins at low ionic strength. Biochemistry. 48, 1954-1963

24 Garcia De La Torre, J., Huertas, M. L. and Carrasco, B. (2000) Calculation of hydrodynamic properties of globular proteins from their atomic-level structure. Biophys J. 78, 719-730

25 Mani, R. S., Boyes, B. E. and Kay, C. M. (1982) Physicochemical and optical studies on calcium- and potassium-induced conformational changes in bovine brain S-100b protein. Biochemistry. 21, 2607-2612 


\section{Chapter 4 \\ STRUCTURAL EFFECTS OF SUBSTITUTED REGIONS IN S100A11}

\subsection{Introduction}

Studies of several EF-hand proteins structures and dynamics have revealed that the proteins exist as an equilibrium of populations between closed and open conformations, which can be altered by $\mathrm{Ca}^{2+}$ and/or target binding [1-3]. Moreover it has been shown that precise amino acid substitutions affect the stability of this equilibrium, favouring the population of one of the two states. [4-7].

Characteristic of the $\mathrm{Ca}^{2+}$-induced open state in the $\mathrm{S} 100$ proteins is the exposure of a large hydrophobic patch that is used to accommodate targets molecules (Figure 1.8). This hydrophobic patch is also present in S100A10 but in a calcium independent manner [8]. It is expected that if any of the chimaeric proteins designed in Chapter 3 are able to favour an open conformation in the absence of $\mathrm{Ca}^{2+}$, as in S100A10, an increase in hydrophobic surface area and a decreased calcium dependence for target binding will be indicative of the transition.

This chapter describes the progressive assays used to determine the effects of the substitutions introduced in the sequence of S100A11 on the open/closed equilibrium of the apo-S100A11 proteins engineered. S100A11 proteins were assessed for increased surface exposure of hydrophobic residues using a hydrophobic probe (ANS) and binding

\footnotetext{
${ }^{1}$ A version of this chapter has been published: Santamaria-Kisiel, L. and Shaw, G.S. 2011 Identification of Regions Responsible for the Open Conformation of S100A10 using Chimaeric S100A11-S100A10 Proteins. Biochem J. 434, 37-48
} 
to a hydrophobic matrix of phenyl Sepharose. Chimeras for which there was evidence of conformational changes were selected and evaluated for calcium-independent target binding to annexin A2 and further characterized using NMR spectroscopy.

Characterization of the chimaeric proteins show that substitutions of residues in the C-terminal loop of S100A11 for those in S100A10, lead to an increase in hydrophobic surface area exposure and binding to annexin A2 in a calcium independent manner. This suggests an important role for loop II in the stabilization of the open conformation of S100A10.

\subsection{Material and Methods}

\subsubsection{Cloning of GST-Annexin A2}

A GST-Annexin A2 fusion protein was constructed by inserting the N-terminal sequence of annexin A2 (STVHEILSKLSLEG) into a GST fusion vector (pGEX-6P1) after the PreScission protease (GE Healthcare) cleavage site. PCR primers that together spanned the peptide sequence were generated with a 27 base pair overlap and ApaI and XhoI restriction sites at the ends (Appendix A). PCR was performed and the product was digested and ligated into the pGEX-6P1 vector. The DNA sequence of the GST-Annexin A2 construct was verified by DNA sequencing at the Robarts Research Institute (London, Ontario).

\subsubsection{Protein Expression and Purification}

S100A11 and S100A10 proteins were expressed and purified as outlined in Chapter 3. GST-Annexin A2 was expressed from bacteria grown in $1 \mathrm{~L}$ of $2 \times \mathrm{YT}$ media 
at $37{ }^{\circ} \mathrm{C}$ to an $\mathrm{OD}_{600}$ of 0.6 and expression was induced by the addition of $0.4 \mathrm{mM}$ IPTG and purified as described for GST-S100A10 in Chapter 3 without tag removal. The fractions containing GST-AnnexinA2 were pooled and dialyzed overnight against $50 \mathrm{mM}$ Tris- $\mathrm{HCl}(\mathrm{pH} 7.5), 0.2 \mathrm{mM}$ EDTA and $0.5 \mathrm{mM}$ TCEP, flash frozen in liquid nitrogen and stored at $-80{ }^{\circ} \mathrm{C}$.

\subsubsection{Fluorescence Experiments}

Hydrophobic surface area exposure of apo-S100A11 chimaeric proteins was analyzed by ANS emission spectra of calcium-free protein samples using a Fluorolog-3 steady-state fluorimeter. Protein samples $(2 \mu \mathrm{M}$ dimer $)$ in $20 \mathrm{mM}$ Tris- $\mathrm{HCl}(\mathrm{pH} 7.2), 10$ $\mathrm{mM} \mathrm{NaCl}, 8 \mu \mathrm{M}$ TCEP and either $100 \mu \mathrm{M}$ EDTA (apo-proteins) or $1 \mathrm{mM} \mathrm{CaCl} 2\left(\mathrm{Ca}^{2+-}\right.$ bound proteins) were saturated with $20 \mu \mathrm{M}$ 4,4'-dianilino-1,1'-binaphthyl-5,5'-disulfonic acid, disodium salt (ANS) and their emission spectra recorded from 430 - $600 \mathrm{~nm}$ using an excitation wavelength of $388 \mathrm{~nm}$ at room temperature. For all measurements five scans were averaged using excitation and emission slits of $0.5 \mathrm{~nm}$ and the buffer background was subtracted. To enable quantitative comparison of ANS fluorescence intensities amongst the different proteins, ANS emission spectra were normalized to the ANS fluorescence maxima for S100A11 in the presence of $1 \mathrm{mM} \mathrm{CaCl}_{2}$.

\subsubsection{Protein Interaction Experiments}

Hydrophobicity of the chimaeric proteins was evaluated as their capacities to bind to the hydrophobic matrix Phenyl Sepharose (CL-4B, GE Healthcare) using a batch method. Calcium-free protein samples (40 $\mu \mathrm{g}$ monomer) in $20 \mathrm{mM}$ Tris- $\mathrm{HCl}(\mathrm{pH} 7.2), 10$ $\mathrm{mM} \mathrm{NaCl}, 0.5 \mathrm{mM}$ TCEP and excess EDTA (or excess $\mathrm{CaCl}_{2}$ ) were incubated with 
phenyl Sepharose beads (200 $\mu \mathrm{l} 50 \%$ slurry) at room temperature for $1 \mathrm{~h}$ with gentle agitation. Proteins that did not interact with the matrix, thereby remaining in the supernatant were recovered by centrifugation (700 g/5 min) and analyzed on $16.5 \%$ TrisTricine SDS-PAGE gels stained with Coomassie-blue. Proteins that remained bound to the matrix were eluted by boiling the beads in SDS-PAGE sample buffer and analyzed as above. To quantify the degree of protein interaction with the hydrophobic matrix, the intensity of the bands in the Coomassie-blue stained SDS-PAGE gels were measured using Image J 1.410 [9] (http://rsb.info.nih.gov/ij/) and their percentages relative to the intensity of total protein (sum of bound and supernatant fractions) determined.

Calcium-insensitive target-binding of the chimaeric proteins was assessed using an annexin A2 GST pull-down assay. GST-Annexin A2 $(620 \mu \mathrm{g})$ was immobilized on glutathione Sepharose beads (600 $\mu \mathrm{L}$ of $50 \%$ slurry, GE Healthcare) and incubated with $40 \mu \mathrm{g}$ of S100A10 or S100A11 proteins in $20 \mathrm{mM}$ Tris- $\mathrm{HCl}(\mathrm{pH} 7.2), 50 \mathrm{mM} \mathrm{NaCl}, 0.5$ mM TCEP and $5 \mathrm{mM}$ EDTA (10 $\mathrm{mM} \mathrm{CaCl}_{2}$ for $\mathrm{Ca}^{2+}-\mathrm{S} 100 \mathrm{~A} 11$, used as a control). After incubation for $30 \mathrm{~min}$ at room temperature with gentle agitation, the beads were spun down at $700 \mathrm{xg}$ for $5 \mathrm{~min}$ and washed 3 times with $1 \mathrm{~mL}$ of the appropriate buffer. Proteins bound to the beads were eluted using $20 \mathrm{mM}$ reduced glutathione, resolved on Tris/Tricine 16.5\% SDS-PAGE gels and detected using Coomassie blue stain. Concentrations were quantified using a series of BSA standards on Coomassie-blue stained SDS-PAGE gels. For all experiments, control samples of apo-S100A11, $\mathrm{Ca}^{2+}-$ S100A11 and S100A10 were included for comparison purposes. All assays were conducted in duplicate with errors found typically within the $10 \%$ range. 


\subsubsection{NMR Spectroscopy}

Structural effects of substitutions in the second calcium-binding site of S100A11 with those in S100A10 (S100A11 ${ }^{\mathrm{LII}}$ mutant) were monitored by NMR spectroscopy using chemical shift mapping and residual dipolar coupling.

\subsubsection{Backbone Chemical Shift Assignment of S100A11 ${ }^{\text {LII }}$}

Experiments were performed on a Varian INOVA $600 \mathrm{MHz}$ spectrometer equipped with pulsed field gradient triple resonance probes. S100A11 ${ }^{\mathrm{LII}}$ samples at a protomer concentration of $1 \mathrm{mM}$ in $20 \mathrm{mM}$ MOPS, $50 \mathrm{mM} \mathrm{KCl}, 5 \mathrm{mM}$ TCEP and $5 \mathrm{mM}$ EDTA at $\mathrm{pH} 7.25$ contained $10 \% \mathrm{D}_{2} \mathrm{O}$ and $33 \mu \mathrm{M}$ DSS as an internal standard. Data for the backbone sequential assignment were collected at $30{ }^{\circ} \mathrm{C}$ and included HNCA [10], HNCACB [11], CBCA(CO)NH [12] and ${ }^{1} \mathrm{H}^{-15} \mathrm{~N}$ HSQC experiments. Spectra were processed with NMRPipe [13] software using a $60^{\circ}$ shifted cosine-squared function in the both dimensions and analyzed using NMRView [14].

\subsubsection{Residual Dipolar Coupling}

Heteronuclear ${ }^{1} \mathrm{H}_{-}{ }^{15} \mathrm{~N}$ residual dipolar coupling were measured from a series of IPAP-HSQC experiments [15] at $30{ }^{\circ} \mathrm{C}$ using a $0.75 \mathrm{mM}$ sample of ${ }^{15} \mathrm{~N}$-labeled apoS100A11 (wild type) in $6.7 \mathrm{mg} / \mathrm{mL}$ Pf1 phage or $1.0 \mathrm{mM}{ }^{15} \mathrm{~N}$-apo-S100A11 ${ }^{\mathrm{LII}}$ in 8.3 $\mathrm{mg} / \mathrm{mL}$ Pf1 phage at $\mathrm{pH}$ 7.2. Identical samples in the absence of phage were used to measure isotropic ${ }^{1} \mathrm{H}-{ }^{15} \mathrm{~N}$ splitting at the same $\mathrm{pH}$ and temperature. In all experiments the proteins were in buffer containing $20 \mathrm{mM}$ MOPS (pH 7.2), $50 \mathrm{mM} \mathrm{KCl}, 5 \mathrm{mM}$ TCEP and $5 \mathrm{mM}$ EDTA. Spectra were processed with NMRPipe [13] software using a $60^{\circ}$ shifted cosine-squared function in the ${ }^{1} \mathrm{H}$ and ${ }^{15} \mathrm{~N}$ dimensions and analyzed using 
NMRView [14]. Components of the molecular alignment tensor and coordinates were determined by using the Dynamo (NMRPipe) program [13].

\subsection{Results and Discussion}

S100A11 undergoes a large calcium-induced conformational change involving the reorientation of helix III in calcium-binding site II [16]. This "opening" of site II forms a hydrophobic cleft comprised of residues from helices III, IV and the helix II-III linker, and helix I' from the partner protomer essential for interaction with annexins A1 and A2 (Figure 1.11). In contrast, S100A10 adopts an open conformation in the absence of calcium. The regions that contribute to and stabilize the open conformation of S100A10 in the absence of calcium were investigated using a series of chimaeric S100A11 proteins.

\subsubsection{Evidence for a $\mathrm{Ca}^{2+}$-independent hydrophobic surface in site II chimaeric proteins}

Since an increase in hydrophobic surface area exposure is indicative of the presence of the open structure, two biochemical assays were used to evaluate the exposed hydrophobic surface area in apo-S100A11, $\mathrm{Ca}^{2+}-\mathrm{S} 100 \mathrm{~A} 11, \mathrm{~S} 100 \mathrm{~A} 10$ and the seven chimaeric proteins. The first assay utilized the extrinsic fluorophore ANS, which is commonly used as an indicator of protein folding, conformational changes and any other events that modify protein surface exposure to solvent. ANS exhibits weak fluorescence in solution but shows greatly enhanced fluorescence upon binding to a hydrophobic protein surface such as those exhibited by the calcium-bound states for S100B [17], troponin C [18] and calmodulin [19]. In the presence of apo-S100A11 ANS showed weak fluorescence near $520 \mathrm{~nm}$ (Figure 4.1), whereas S100A10 or $\mathrm{Ca}^{2+}-\mathrm{S} 100 \mathrm{~A} 11$ caused a 
dramatic increase in fluorescence, accompanied by a blue shift to about $500 \mathrm{~nm}$, indicative of ANS binding to a exposed hydrophobic surface in each protein. Interestingly, the fluorescence for $\mathrm{S} 100 \mathrm{~A} 10$ was about $50 \%$ higher than for $\mathrm{Ca}^{2+}$ S100A11 (Figure 4.2), indicating that a larger number of ANS molecules were binding to the surface of S100A10. This is consistent with the exposed hydrophobic surface area in S100A10 which is $\sim 200 \AA$ greater than that in $\mathrm{Ca}^{2+}-\mathrm{S} 100 \mathrm{~A} 11$ based on their threedimensional structures.

The chimaeric S100A11 proteins were examined in the absence of calcium to determine if the substitutions used caused a conformational change that might liberate a greater hydrophobic surface area. For proteins with substitutions in the calcium-binding sites, a wide range of responses was noted in the ANS fluorescence spectra (Figures 4.1 and 4.2). The S100A $11^{\mathrm{LI}}$ protein showed similar fluorescence spectrum to that of apoS100A11 suggesting that changes to calcium-binding site I had little effect on the hydrophobicity of the protein. This is consistent with NMR data that showed apoS100A $11{ }^{\mathrm{LI}}$ had a ${ }^{1} \mathrm{H}^{-}{ }^{15} \mathrm{~N}$ HSQC spectrum that was very similar to apo-S100A11 (Figure 3.7B). On the other hand, chimaeric proteins that had modifications in site II $\left(\mathrm{S} 100 \mathrm{~A} 11^{\mathrm{N} 66 \mathrm{C}, \mathrm{E} 75 \mathrm{~S}}, \mathrm{~S} 100 \mathrm{~A} 11^{\mathrm{LII}}, \mathrm{S} 100 \mathrm{~A} 11^{\mathrm{LI}, \mathrm{LII}}\right)$ all showed $3-5$ fold increases in fluorescence and an accompanying blue shift (Figures 4.1 and 4.2) compared to apoS100A11. These observations are indicative of a greater hydrophobic surface exposure, that can result from either a "molten globular" form of the S100A11 constructs containing substitutions in site II or a greater ability to occupy an open conformation for these proteins. 


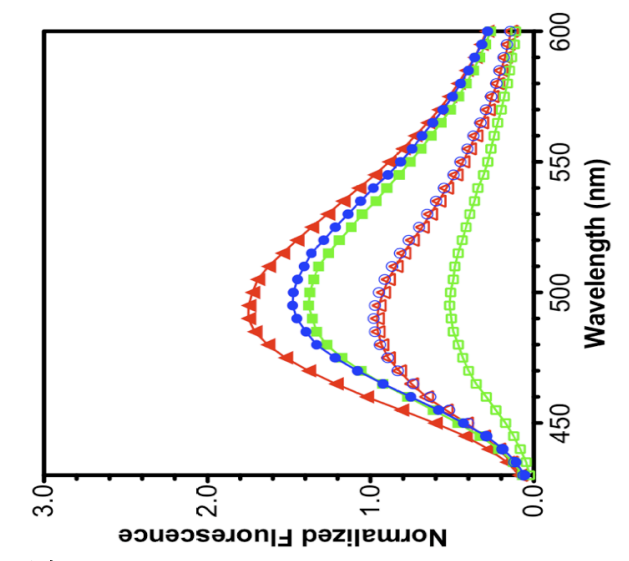

u

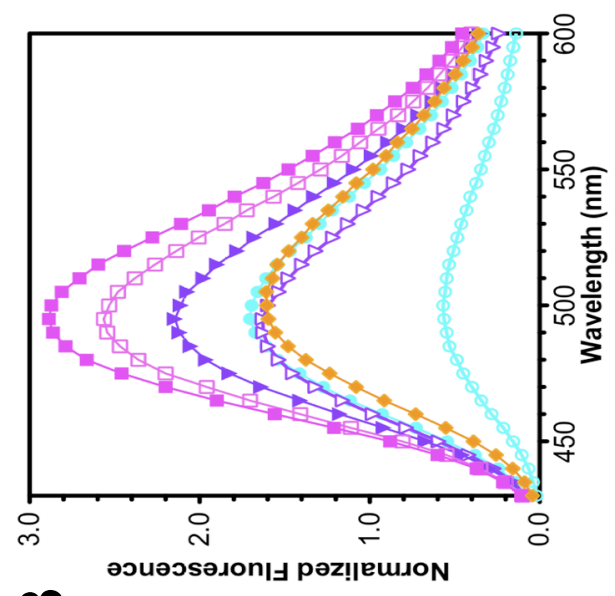

$m$

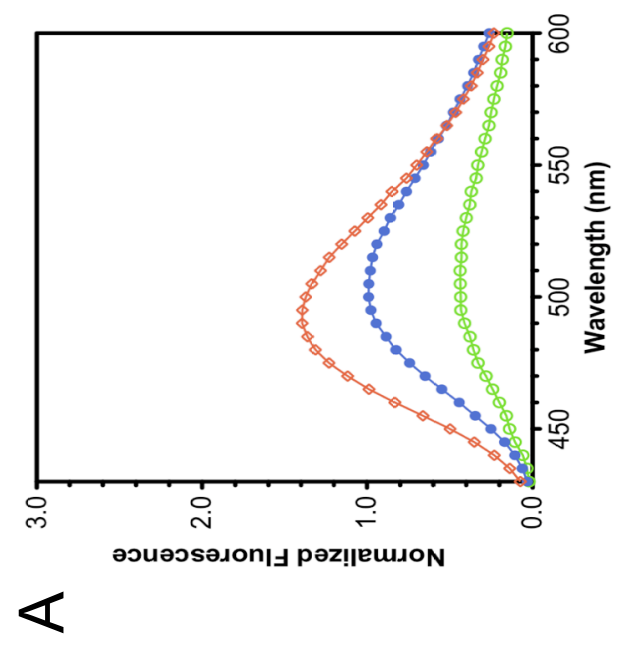

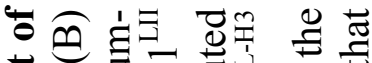

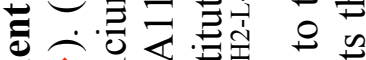

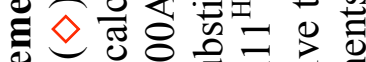

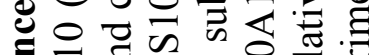

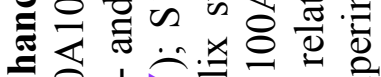

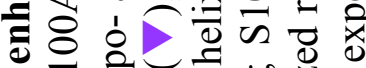

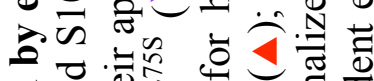

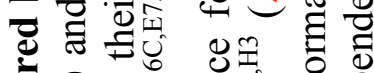
อ. 的

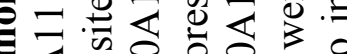

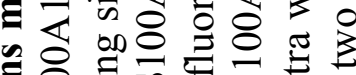

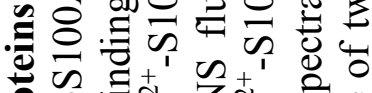

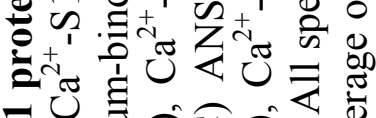

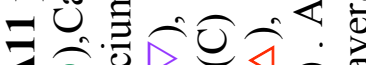

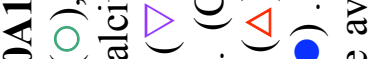
8 造

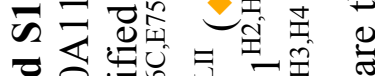
영 元으ㅇㅛㅛ 의

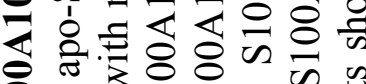

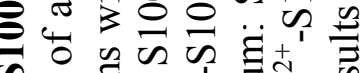
a $\cdot \cdots+1$ 言 ப.

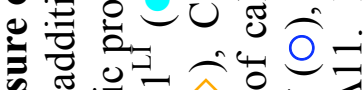

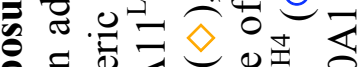
웡

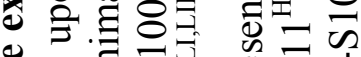
\& 긍 的

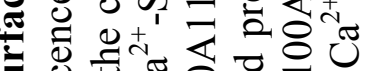

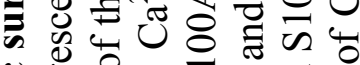
$\because \tilde{0} \dot{0} \bar{\sim}$ o

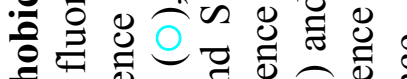

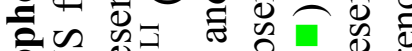

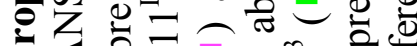

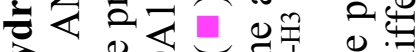

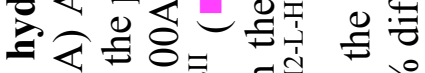

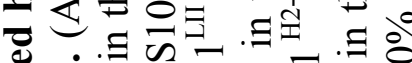

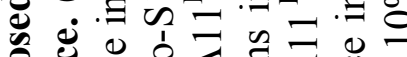

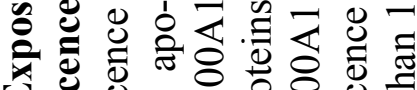
荬苾 $\rightarrow$ 인

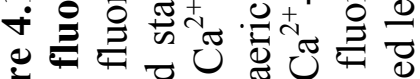

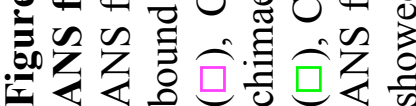




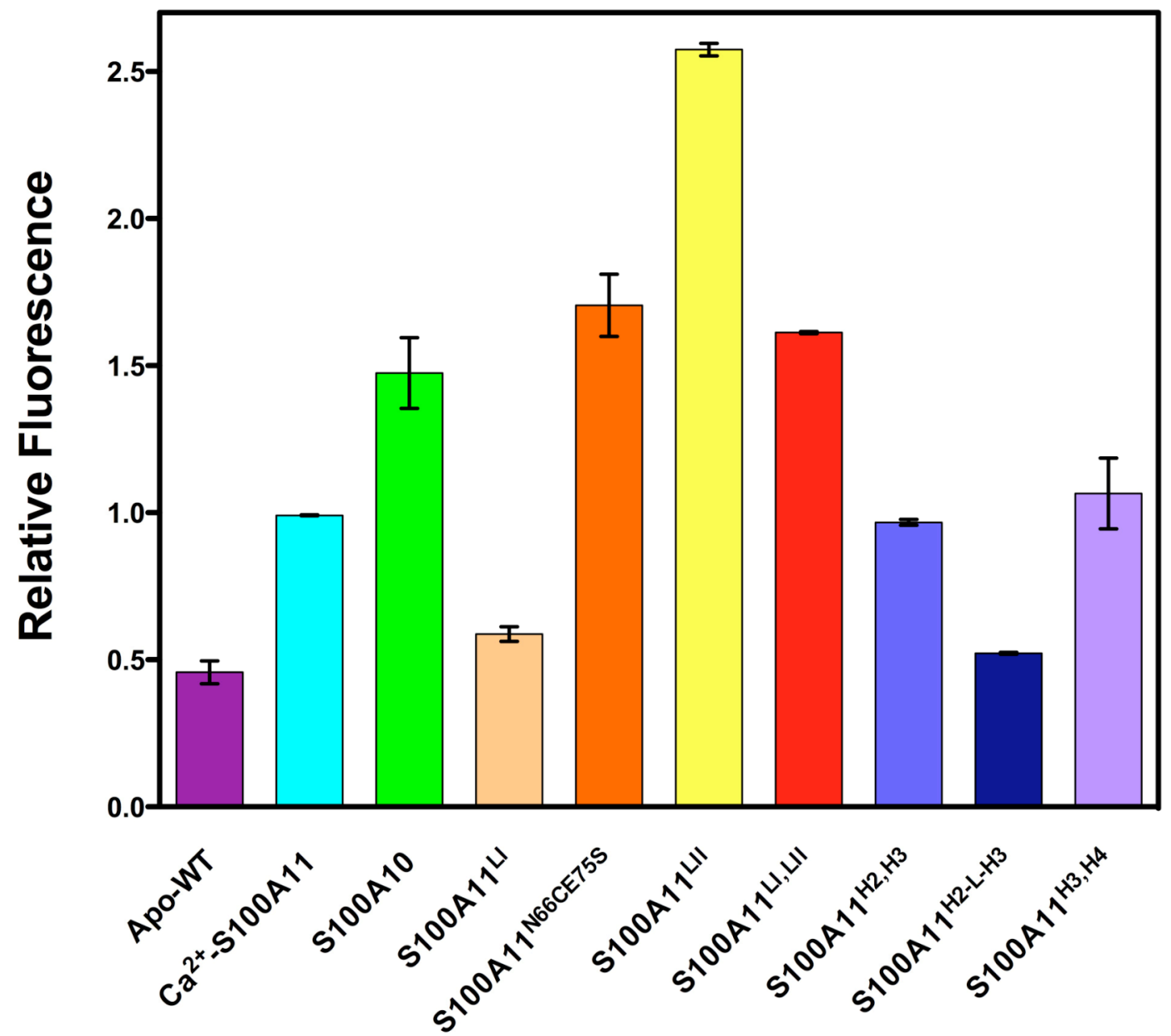

Figure 4.2 Observed changes in ANS fluorescence intensity of apo-S100A11 proteins.

Maximum fluorescence intensity of ANS in the presence of apo-S100A11 proteins, as labeled in the figure, was normalized to the ANS in the presence of $\mathrm{Ca}^{2+}-\mathrm{S} 100 \mathrm{~A} 11$ and plotted as a bar graph. In the case of S100A11 with substitutions in the second calciumbinding site the ANS fluorescence shows a large increase in intensity compared with substitutions in loop I or the linker regions. This is consistent with a greater hydrophobic surface exposure, likely from a larger population in the open state as a result of the amino acid substitutions. The bar graph is an average of two independent experiments. Errors bars represent standard deviation. 
Studies of molten globular intermediate states in protein folding have shown significant increases in the ANS fluorescence are due to the exposure of hydrophobic core regions that are unmasked to the dye in the folded structure [20,21]. However it is unlikely that this is the cause of the increased fluorescence observed in the chimaeric proteins with modified site II, since the S100 proteins have been shown to be remarkably stable and the chimaeric proteins did not exhibit any non-native molten globule-like properties during the process of purification or characterization by NMR spectroscopy (Figure 3.13).

The S100A11 ${ }^{\text {LI }}$ chimaeric protein had the largest increase in fluorescence. However, the S100A $11^{\mathrm{N} 66 \mathrm{C}, \mathrm{E} 75 \mathrm{~S}}$ protein had about $70 \%$ of the fluorescence change observed in $\mathrm{S} 100 \mathrm{~A} 11^{\mathrm{LII}}$, indicating that positions $\mathrm{N} 66$ and E75 are potentially key residues for the structural difference in S100A10 compared to apo-S100A11, while other residues in the loop contribute to a lesser extent. Again this is consistent with the ${ }^{1} \mathrm{H}^{15} \mathrm{~N}$ HSQC spectra that showed S100A11 ${ }^{\mathrm{N} 66 \mathrm{C}, \mathrm{E} 75 \mathrm{~S}}$ had a large number of peaks with chemical shift changes that are not spatially close to the N66C and E75S substitutions (Figure 3.13 A). Additional substitutions in the first calcium-binding loop along with those in site II appeared to have a moderating effect on hydrophobic surface exposure since the S100A $11^{\mathrm{LI}, \mathrm{LII}}$ protein displayed increased fluorescence compared to S100A11 ${ }^{\mathrm{LI}}$ or apoS100A11, but lower than S100A11 ${ }^{\text {LII }}$. The results also show that substitutions in both EFhands do not result in structural changes that are simply additive. Since this protein has lost its ability to bind calcium, very little change was noted in its ANS fluorescence spectrum upon calcium addition. However, the remaining calcium-binding site chimeras 
all exhibited increased fluorescence intensities ranging from about 4-fold for S100A11 ${ }^{\mathrm{LI}}$ to about $15 \%$ for S100A $11^{\mathrm{LII}}$.

Substitutions of helices II, III or IV in S100A11 resulted in more modest fluorescence changes (Figures 4.1C and 4.2) compared to the calcium-binding loop substitutions. For example, both the $\mathrm{S} 100 \mathrm{~A} 11^{\mathrm{H} 3, \mathrm{H} 4}$ and $\mathrm{S} 100 \mathrm{~A} 11^{\mathrm{H} 2, \mathrm{H} 3}$ chimaeric proteins had increased fluorescence intensities compared to apo-S100A11 and similar to $\mathrm{Ca}^{2+}$ S100A11 indicating an increase in hydrophobic surface area. The S100A11 ${ }^{\mathrm{H}-\mathrm{L}-\mathrm{H} 3}$ protein had a fluorescence spectrum that was more similar to apo-S100A11 and S100A1 $1^{\mathrm{LI}}$ indicating S100A11 ${ }^{\mathrm{H} 2-\mathrm{L}-\mathrm{H} 3}$ adopted a structure more similar to apo-S100A11 having a minimally exposed hydrophobic surface. Although the S100A11 ${ }^{\mathrm{H} 3, \mathrm{H} 4}$ and $\mathrm{S} 100 \mathrm{~A} 11^{\mathrm{H} 2, \mathrm{H} 3}$ chimaeric proteins showed increased ANS fluorescence, these changes were consistently lower than observed for the site II substituted proteins (S100A11 ${ }^{\mathrm{LII}}, \mathrm{S} 100 \mathrm{~A} 11^{\mathrm{N} 66 \mathrm{C}, \mathrm{E} 75 \mathrm{~S}}$ ). Upon addition of calcium, all the chimaeric helix proteins exhibited $>50 \%$ increases from their apo states indicating a greater hydrophobic surface as a result of the calciuminduced conformational change. Overall, these assays showed that the largest contribution to the increased hydrophobic surface observed in S100A10 is produced from substitutions in its second calcium-binding loop, consistent with a stabilization of the open conformation.

Although ANS binding is a qualitative test, the experiments suggested that replacement of a pair of residues $\left(\mathrm{S} 100 \mathrm{~A} 11^{\mathrm{N} 66 \mathrm{C}, \mathrm{E} 75 \mathrm{~S}}\right)$ or the entire second calcium-binding loop of S100A11 (S100A11 $\left.{ }^{\mathrm{LII}}\right)$ with the corresponding residues from S100A10 resulted in the exposure of a hydrophobic surface in these proteins in the calcium-free state. To 
identify if this resulted from an altered open/closed equilibrium that stabilizes the open state, similar to that observed upon calcium binding to S100A11, the capacity for binding of the S100A11 chimaeric proteins to a hydrophobic phenyl Sepharose matrix was evaluated (Figure 4.3). Many EF-hand proteins, including S100A11, preferentially bind to phenyl Sepharose in the calcium-bound form using an exposed hydrophobic surface to interact with the matrix. This method has been used not only to show a calcium-sensitive conformational change but also as evidence for target protein interactions. In the absence of calcium, S100A11 did not bind to the phenyl Sepharose beads (Figures 4.3 A and 4.4). In contrast, $\mathrm{Ca}^{2+}-\mathrm{S} 100 \mathrm{~A} 11$ and $\mathrm{S} 100 \mathrm{~A} 10$ both bound strongly to the beads, with a minimal amount of material found in the supernatant. For S100A11 this indicated the protein had adopted an altered conformation with an exposed hydrophobic surface upon binding of calcium. As with the ANS experiments, the chimaeric S100A11 proteins showed a range of binding efficiencies to the matrix although all of the chimaeric proteins (Figures $4.3 \mathrm{C}$, D) exhibited lower phenyl sepharose binding compared to $\mathrm{Ca}^{2+}$ S100A11 and S100A10 (Figure 4.4). Three chimaeric proteins (S100A11 ${ }^{\mathrm{LI}}, \mathrm{S} 100 \mathrm{~A} 11^{\mathrm{H} 2-\mathrm{L}-}$ ${ }^{\mathrm{H} 3}, \mathrm{~S} 100 \mathrm{~A} 11^{\mathrm{H} 3, \mathrm{H} 4}$ ) bound poorly to phenyl Sepharose in the absence of calcium, but like S100A11 the interaction was increased upon calcium binding, indicating that binding of calcium displaces the equilibrium towards the open structure. The S100A11 ${ }^{\text {LI,LII }}$ chimera showed weak phenyl Sepharose binding that was not altered upon calcium addition, consistent with the inactivation of both calcium-binding sites in this protein.

In contrast, when residues in site II of S100A11 were replaced for those in S100A10 (S100A11 $\left.{ }^{\mathrm{N} 66 \mathrm{C}, \mathrm{E} 75 \mathrm{~S}}, \mathrm{~S} 100 \mathrm{~A} 11^{\mathrm{LII}}\right)$ a large increase in bound protein was observed 


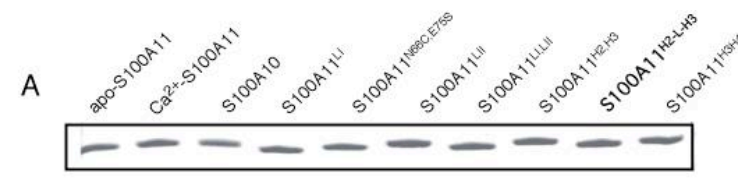

B

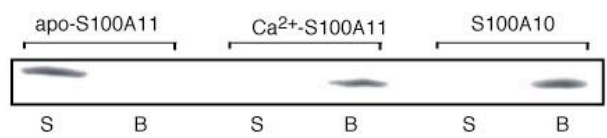

C
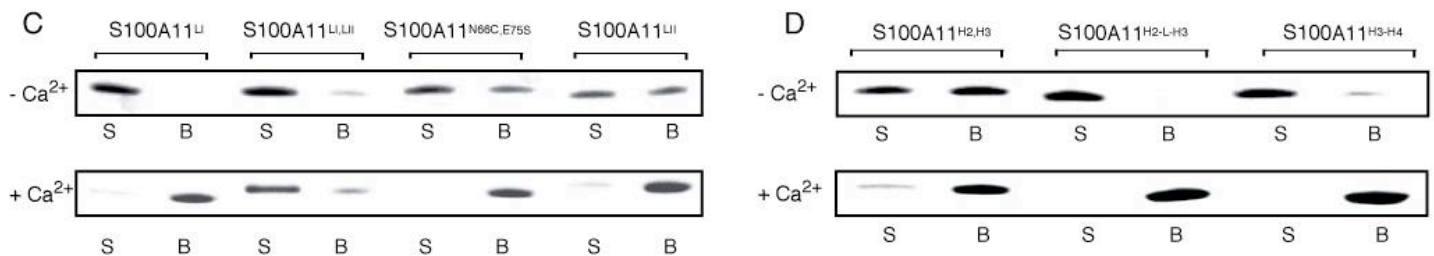

Figure 4.3 Exposed hydrophobic surface area of S100A10 and S100A11 proteins evaluated by their binding capacities to a phenyl Sepharose matrix.

Binding to phenyl Sepharose for apo-S100A11, $\mathrm{Ca}^{2+}-\mathrm{S} 100 \mathrm{~A} 11$ and calcium-free S100A10, (C) chimaeric S100A11 proteins with modified calcium-binding sites in the absence $\left(-\mathrm{Ca}^{2+}\right)$ and in the presence of calcium $\left(+\mathrm{Ca}^{2+}\right)$, (D) chimaeric S100A11 proteins with substituted helical regions in their apo- $\left(-\mathrm{Ca}^{2+}\right)$ and calcium loaded states $\left(\mathrm{Ca}^{2+}\right)$. Proteins were identified in the supernatant $(\mathrm{S})$ and bound $(\mathrm{B})$ fractions to the beads after extensive washes using SDS-PAGE and Coomassie-blue staining. (A) Loading controls for each protein as described in Experimental section. Proteins with modified site II show a significant increase in protein bound to the phenyl Sepharose beads in the absence of calcium, indicative of a larger hydrophobic surface compared to that of apo-S100A11. This is consistent with increased stability of these proteins to occupy an open conformation. 


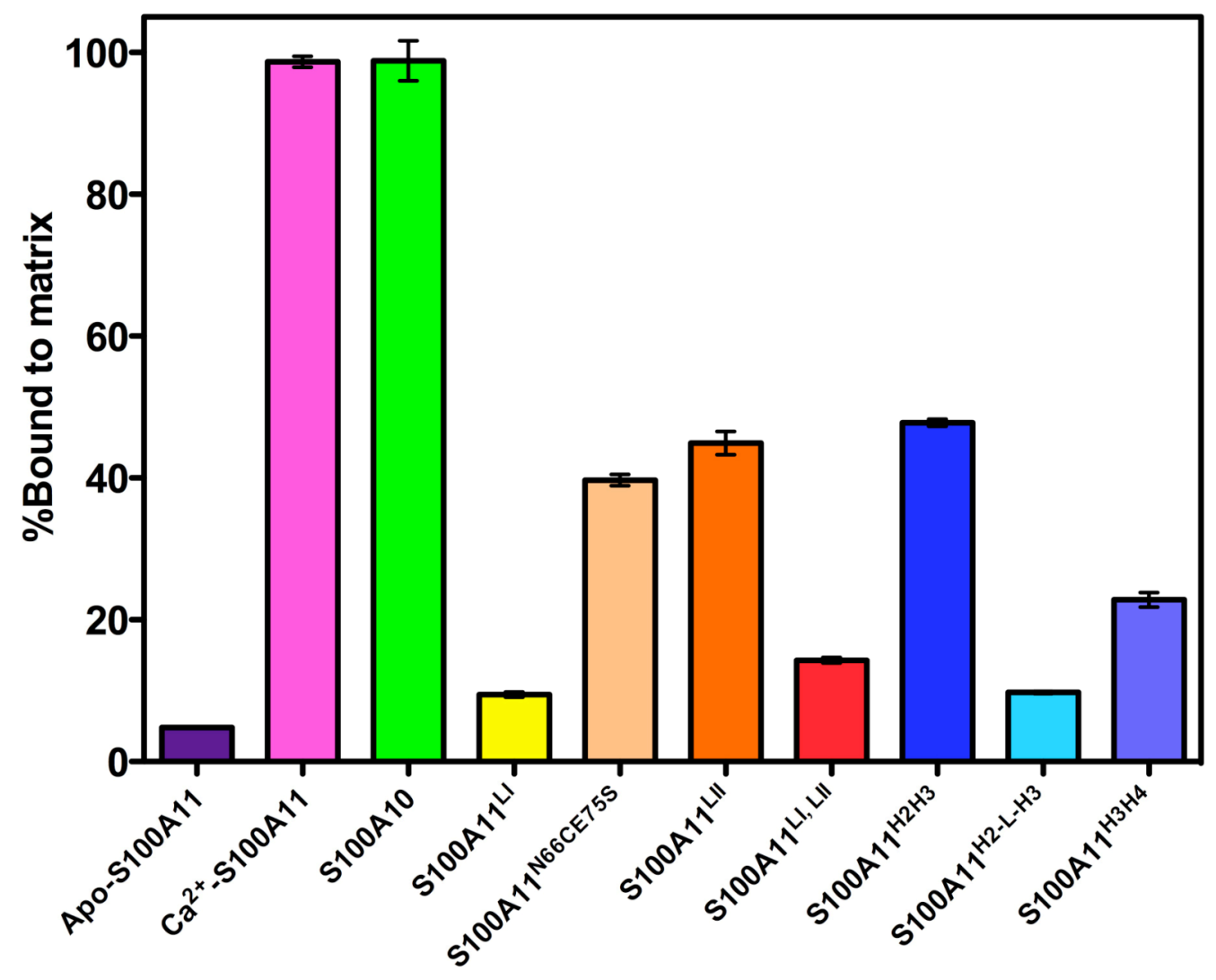

Figure 4.4 Degree of apo-S100A11 proteins interaction with a phenyl Sepharose matrix.

The intensity of the bands in the Coomassie-blue stained SDS gels from the interactions of the apo-S100A11 proteins with a hydrophobic matrix were measured (Image J http://rsb.info.nig.gov/.ij/) to quantify the degree of protein interaction. Three S100A11 proteins presented enhanced interaction with the matrix: S100A11 ${ }^{\mathrm{N} 66 \mathrm{C}, \mathrm{E} 75 \mathrm{~S}}, \mathrm{~S} 100 \mathrm{~A} 11^{\mathrm{LII}}$ and $\mathrm{S} 100 \mathrm{~A} 11^{\mathrm{H} 2}$, H3 compared to apo-S100A11 but not nearly as efficient as in $\mathrm{Ca}^{2+}$ S100A11 or S100A10. The bar graph is an average of two independent experiments. Errors bars represent standard deviation. 
in the calcium-free states (Figure $4.3 \mathrm{C}$ ) consistent with each of these proteins exposing a greater hydrophobic surface than apo-S100A11. Upon calcium addition the proportion of these proteins bound to the beads was enhanced indicating that calcium binding was able to further alter the open-state to closed state equilibrium and consequently the exposed hydrophobic surface. The $\mathrm{S} 100 \mathrm{~A} 11^{\mathrm{H} 2, \mathrm{H} 3}$ protein behaved in a similar fashion, the only S100A11 chimera having substitutions in the helical regions that also showed an increase in ANS fluorescence. These results indicate that although both ANS fluorescence and phenyl Sepharose binding are good indicators of exposed hydrophobic surface, subtle differences exist between the two methods. Nevertheless, apoS100A $11^{\text {N66C,E75S }}$, apo-S100A1 $1^{\mathrm{LII}}$ and apo-S100A11 ${ }^{\mathrm{H} 2, \mathrm{H} 3}$ consistently showed these chimaeric proteins had a greater exposed hydrophobic surface than apo-S100A11, likely due to increase in the ability of these mutants to occupy an open conformation, similar to that observed in S100A10 or upon calcium binding to S100A11.

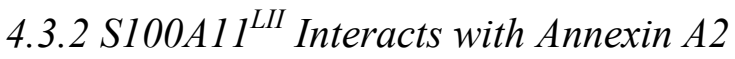

The increased exposure of hydrophobic surface in S100A11 ${ }^{\text {LII }}$ and S100A $11^{\text {N66C,E75S }}$, indicated by ANS and phenyl Sepharose experiments, may be adequate for target protein binding to these proteins in the calcium-free state. It has been shown that an $\mathrm{N}$-terminal peptide from the phospholipid-binding protein annexin $\mathrm{A} 2$ can bind to $\mathrm{Ca}^{2+}-\mathrm{S} 100 \mathrm{~A} 11(\mathrm{Kd} \sim 3 \mu \mathrm{M})$, but not in the absence of calcium [22]. This sequence can also interact tightly with S100A10 in the absence of calcium since S100A10 adopts a similar structure to $\mathrm{Ca}^{2+}$-S100A11. Using a GST construct with the Nterminal 14 residues of annexin A2, the ability of binding to S100A11, S100A10 and the 
chimaeric proteins was evaluated. As expected, apo-S100A11 did not interact with GST-Annexin A2 but showed tight binding in the presence of calcium (Figure 4.5), a similar effect to that noted for S100A10 in the absence of calcium. Of the four chimaeric S100A11 proteins that showed the highest hydrophobic surface area by ANS and phenyl Sepharose experiments in the absence of calcium, three proteins (S100A1 $1^{\mathrm{N} 66 \mathrm{C}, \mathrm{E} 75 \mathrm{~S}}$, $\mathrm{S} 100 \mathrm{~A} 11^{\mathrm{H} 2, \mathrm{H} 3}, \mathrm{~S} 100 \mathrm{~A} 11^{\mathrm{H} 3, \mathrm{H} 4}$ ) repeatedly showed no detectable binding to annexin $\mathrm{A} 2$. This indicated that the increased hydrophobicity for these proteins was not sufficient for annexin A2 interaction. It also suggests that additional stabilizing factors are needed since the binding of the target did not displace the equilibrium further towards the open form. Conversely, apo-S100A11 ${ }^{\mathrm{LII}}$ displayed a reproducible interaction with annexin A2, albeit weaker than either S100A10 or $\mathrm{Ca}^{2+}-\mathrm{S} 100 \mathrm{~A} 11$, indicating that the site II loop region provides important contributions for stabilization of the open state of the protein and its interaction with annexin A2. Interestingly, target binding did not shift the equilibrium further towards the open state, as evidenced in the weaker binding of annexin A2 to apo-S100A11 ${ }^{\text {LII }}$ compared to $\mathrm{Ca}^{2+}-\mathrm{S} 100 \mathrm{~A} 11$ or S100A10. This could imply that further substituions are needed to destabilize the closed form of the chimaeric protein.

In summary, the overall trend of the chimaeric proteins with substitutions in the C-terminal loop (S100A $11^{\mathrm{LII}}$ and S100A $11^{\mathrm{N} 66 \mathrm{C}, \mathrm{E} 75}$ ) is an increase in hydrophobic surface exposure, consistent with an apparent shift in the closed/open equilibrium towards the open form in the absence of calcium. 


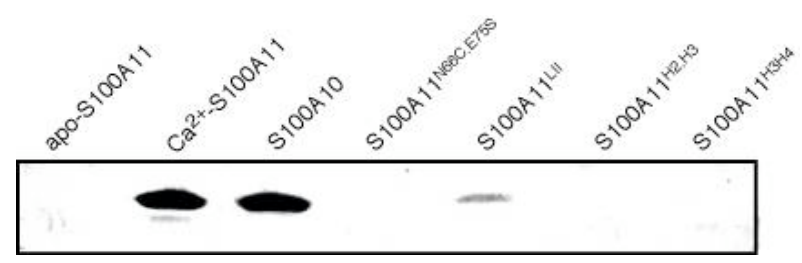

Figure 4.5 Interaction of S100A11 and S100A10 proteins with annexin A2.

For each protein, GST-annexin A2 $(620 \mu \mathrm{g})$ was immobilized on glutathione-Sepharose beads and incubated with $40 \mu \mathrm{g}$ of the corresponding S100 protein. The supernatant was removed and the beads washed extensively prior to elution with glutathione and analyzed by SDS-PAGE and Coomassie-blue staining for bound S100 protein. 


\subsubsection{Structural effects of substitutions in S100A11 $1^{\text {LII }}$}

\subsubsection{Sequential backbone resonance assignment of S100A1 $1^{\text {LII }}$}

The three-dimensional structure of apo-S100A11 has been determined by NMR spectroscopy and it was used as a reference structure to evaluate the effects in S100A11 due to substitutions introduced in the second calcium-binding loop (S100A11 $\left.{ }^{\mathrm{LII}}\right)$. Amino acid substitutions in $\mathrm{S} 100 \mathrm{~A} 11^{\mathrm{LII}}$ resulted in an ${ }^{1} \mathrm{H}-{ }^{15} \mathrm{~N}-\mathrm{HSQC}$ spectrum considerably different from that of the parental protein (Figure 4.6), requiring an ab initio assignment of the polypeptide backbone of chimaeric S100A11 ${ }^{\mathrm{LII}}$ using multidimensional NMR experiments.

The ${ }^{1} \mathrm{H}-{ }^{15} \mathrm{~N}$ HSQC spectrum of recombinant apo-S100A1 ${ }^{\text {LII }}$ is shown in Figure 4.6 with 91 of the 97 backbone amide proton/nitrogen resonances identified. The unambiguous assignment of the backbone of apo-S100A11 ${ }^{\mathrm{LII}}$ was completed using HNCACB and $\mathrm{CB}(\mathrm{CA}) \mathrm{CONH}$ experiments, which correlate the backbone amide proton and nitrogen of residue $i$, with the intraresidue $\mathrm{C} \alpha, \mathrm{C} \beta$; and the preceding residue (i-1) $\mathrm{C} \alpha-1, \mathrm{C} \beta-1$. In addition, an HNCA experiment, which correlates the backbone amide proton and nitrogen resonances with the intraresidue $C \alpha$ and $C \alpha-1$ were used to aid the assignments. Regions of the $\mathrm{HNCACB}$ and $\mathrm{CB}(\mathrm{CA}) \mathrm{CONH}$ spectra illustrating the sequential assignment of residues T33 to F35 are shown in Figure 4.7.

\subsubsection{Chemical Shift differences}

Apo-S100A11 ${ }^{\mathrm{LII}}$ was the only chimaeric protein tested that exhibited increased hydrophobicity compared to apo-S100A11, and the ability to bind to annexin A2. 


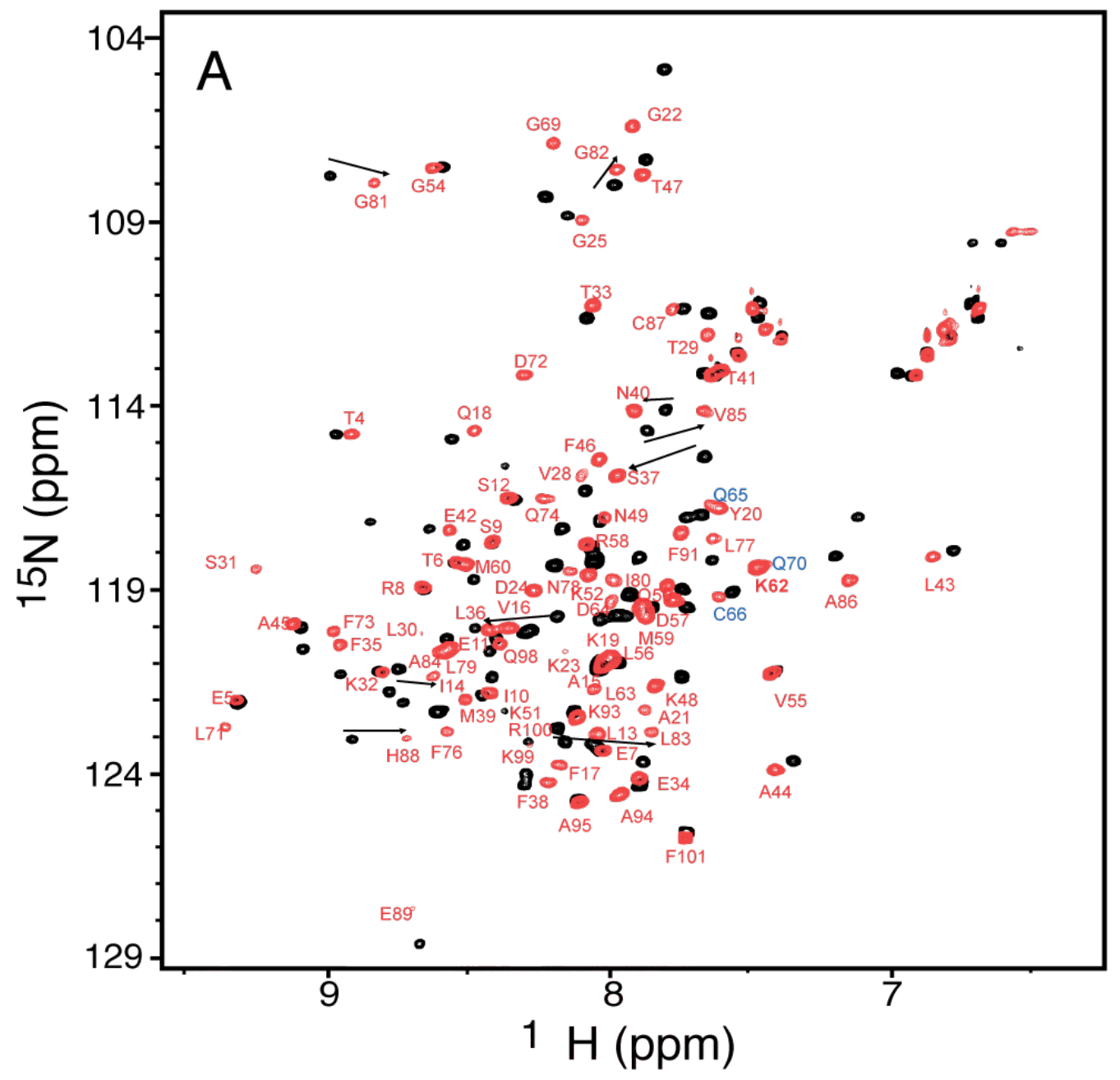

Figure 4.6 ${ }^{1} \mathrm{H}-{ }^{15} \mathrm{~N}$ HSQC spectrum of apo-S100A11 ${ }^{\mathrm{LII}}$.

Overlay of ${ }^{1} \mathrm{H}^{15}{ }^{\mathrm{N}} \mathrm{HSQC}$ spectra of apo-S100A11 (black) and S100A11 ${ }^{\mathrm{LII}}$ (red). Residues that experienced the largest chemical shift differences between the two proteins are indicated by arrows. 


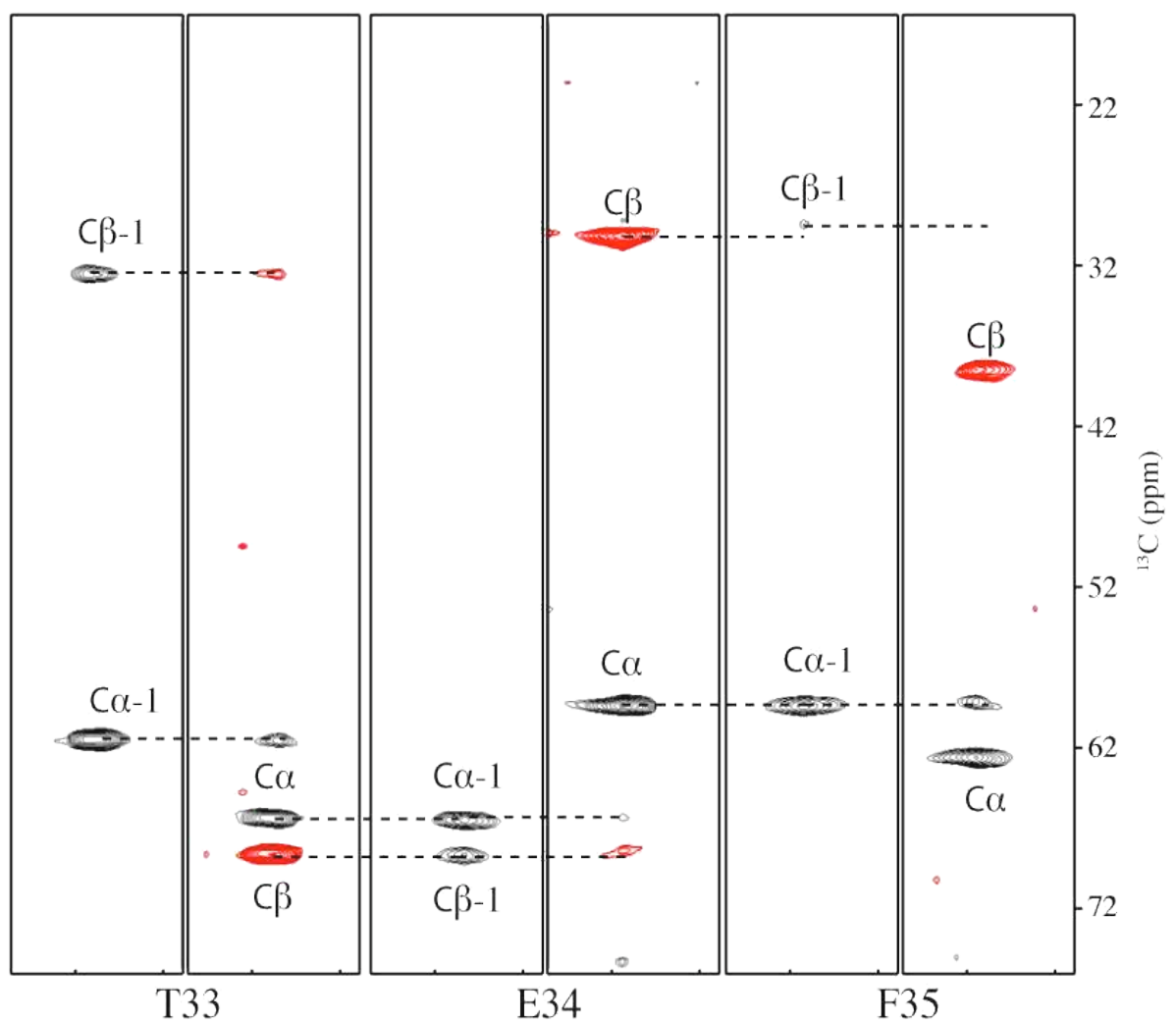

Figure 4.7 Sequential backbone assignment of S100A11 ${ }^{\mathrm{LII}}$.

The spectra show ${ }^{15} \mathrm{~N}$ planes for residue T33-F35 in helix II of S100A1 ${ }^{\text {LII }}$. For each pair of planes, the CBCA $(\mathrm{CO}) \mathrm{NH}$ is shown on the left and the HNCACB is shown on the right. For each residue the $\mathrm{C} \alpha$ and $\mathrm{C} \beta$ are shown on the HNCACB spectrum and the corresponding $\mathrm{C} \alpha$ and $\mathrm{C} \beta$ for the previous residue (i-1) are shown on the $\mathrm{CBCA}(\mathrm{CO}) \mathrm{NH}$. The spectra were collected at $30{ }^{\circ} \mathrm{C}$ on a $1.0 \mathrm{mM} \mathrm{S100A} 11^{\mathrm{LII}}$ sample in $90 \% \mathrm{H}_{2} \mathrm{O} / 10 \%$ $\mathrm{D}_{2} \mathrm{O}$ containing $20 \mathrm{mM}$ MOPS ( $\mathrm{pH} 7.25$ ), $50 \mathrm{mM} \mathrm{KCl,} 5 \mathrm{mM}$ TCEP and $5 \mathrm{mM}$ EDTA. 
This suggests that substitutions in site II are favouring the population of the open state in S100A $11^{\text {LII }}$ similar to either $\mathrm{Ca}^{2+}-\mathrm{S} 100 \mathrm{~A} 11$ or S100A10. To identify if a shift towards the open state has occurred for S100A11 ${ }^{\mathrm{LII}}$, the chemical shift perturbation method was used, comparing its ${ }^{1} \mathrm{H}_{-}{ }^{15} \mathrm{~N}-\mathrm{HSQC}$ spectrum with that of apo-S100A11 (Figure 4.8A). A similar comparison between $\mathrm{Ca}^{2+-} \mathrm{S} 100 \mathrm{~A} 11$ and apo-S100A11 was also completed (Figure 4.8B). It was anticipated that residues $>6 \AA$ from the sites of the substitutions in S100A11 ${ }^{\text {LII }}$ might exhibit chemical shift changes, resulting from an altered conformation, similar to those observed between $\mathrm{Ca}^{2+-} \mathrm{S} 100 \mathrm{~A} 11$ and apo-S100A11. As expected the data showed that many peaks in site II of apo-S100A11 ${ }^{\mathrm{LII}}$ displayed changes (L63, L65, C66, G69, D72, F73, Q70) where the substitutions were made in S100A11, and in site I (A21, T29, L30, S31) that adjoins this region. Most of these residues also experienced chemical shift changes upon calcium binding to S100A11 although the magnitude of the shifts are more pronounced due to the added electrostatic effect of calcium coordination. Several residues experienced larger than average chemical shift differences between S100A11 ${ }^{\text {LI }}$ and apo-S100A11 that were $>6 \AA$ from the nearest substitution based on the threedimensional structure of apo-S100A11. These included several residues in helix II (L36, S37, N40) and helix IV (G81, G82, L83, A84, V85, H88). An analogous comparison between $\mathrm{Ca}^{2+-} \mathrm{S} 100 \mathrm{~A} 11$ and apo-S100A11 (Figure 4.8B) revealed the calcium-induced conformational change affected many residues remote from the calcium-binding sites including I14, V16 (helix I), L36 (helix II), A44, T47-K51 (linker) and G82, V85 and C87 (helix IV). This analysis revealed some similarity existed for perturbed residues in S100A11 ${ }^{\text {LII }}$ (Figure 4.8A) and $\mathrm{Ca}^{2+-} \mathrm{S} 100 \mathrm{~A} 11$ (Figure 4.8B) when compared to calcium- 

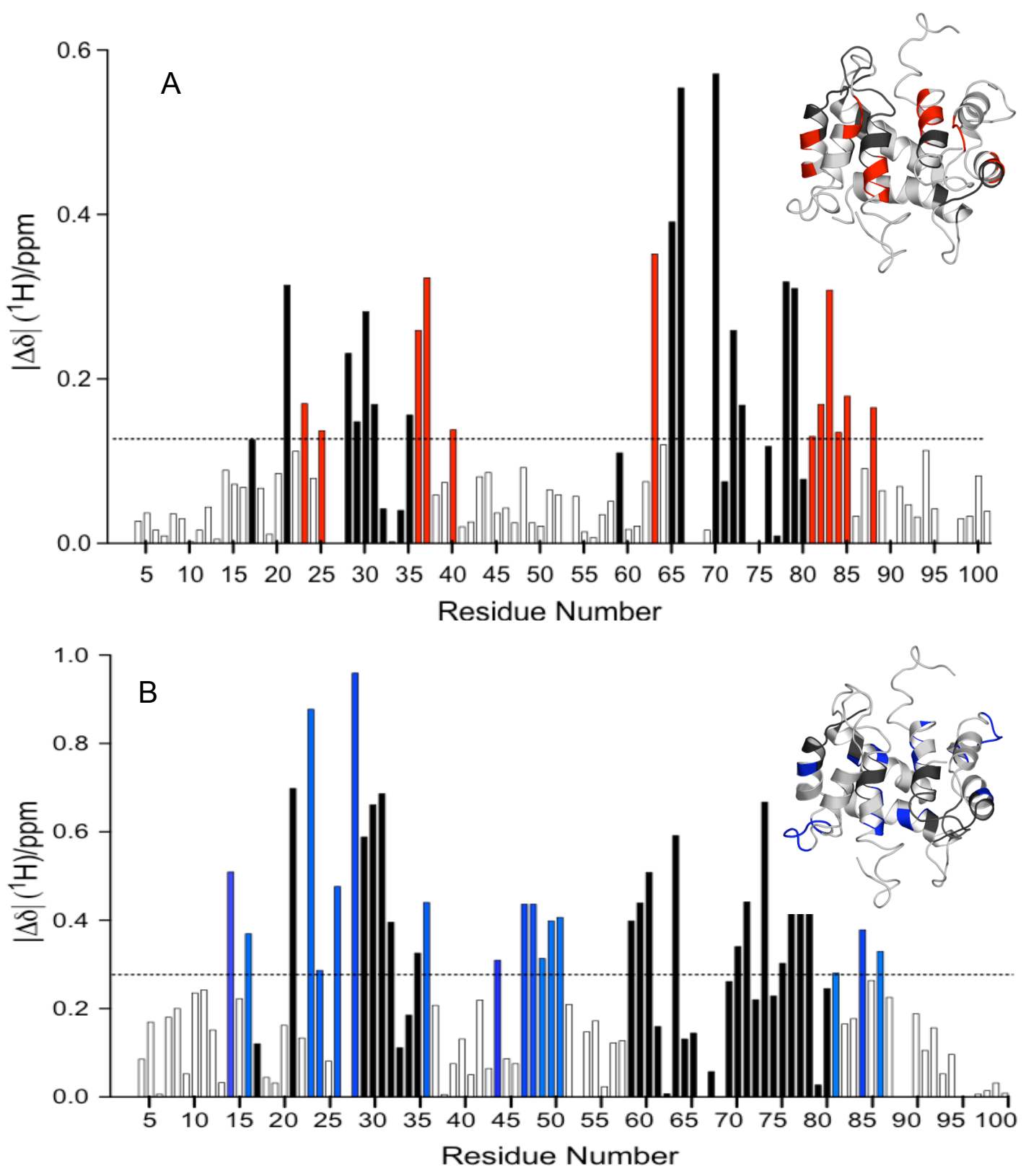

Figure 4.8 Structural changes in S100A11 ${ }^{\mathrm{LII}}$ compared to apo-S100A11

Chemical shift differences for amide protons between (A) S100A1 $1^{\mathrm{LII}}$ and apo-S100A11 and (B) $\mathrm{Ca}^{2+}$ S100A11 and apo-S100A11. Filled bars correspond to residues in close proximity $(<6 \AA)$ from the substitution sites in S100A11 ${ }^{\text {LII }}$. Dotted lines are shown to indicate the average change in chemical shift. Residues that presented the largest chemical shift differences (above average) between the two proteins are highlighted in red for apo-S100A1 ${ }^{\text {LII }}$ and blue for $\mathrm{Ca}^{2+}-\mathrm{S} 100 \mathrm{~A} 11$ on the three-dimensional structure of apo-S100A11. 
free S100A11 especially with respect to residues affected in helices II and IV. However, it was clear that a large segment in the linker region (T47-K51) and the C-terminus of helix I (I14, V16) showed much larger changes upon calcium-binding to S100A11 compared to apo-S100A11 ${ }^{\text {LII }}$. Most of these residues also exhibit large chemical shift changes upon interaction with annexin A2, indicating their importance in $\mathrm{Ca}^{2+}-\mathrm{S} 100 \mathrm{~A} 11$.

Overall this analysis shows that substitution of residues in the second calciumbinding site of S100A11 with those from S100A10 affects the environments of residues in helix II, C-terminus of helix III and helix IV but has a smaller impact on residues in helix I and the linker regions that comprise a major portion of the binding site for annexin A2. While similarity in chemical shifts between S100A11 LII and the apo-state of S100A11 suggest that the open state was not fully reached, similarities with the changes in chemical shift seen in the open form of calcium- bound S100A11 provides evidence that residues in loop II lead to an increase in the population of the open state in apoS100A $11^{\text {LII }}$, as suggested by the previous hydrophobic and target binding assays.

\subsubsection{Residual Dipolar Coupling}

Dipolar couplings are through-space interactions that take place between any pair of magnetically active nuclei. Tumbling of proteins in solution are isotropic leading to no preference in orientation with respect to the magnetic field and averaging the dipolar coupling to zero. Introduction of aligning molecules in the media such as bicelles, rodshape viruses and filamentous phages can cause alignment of the protein with respect to the magnetic field, introducing an anisotropic component that gives rise to measurable dipolar couplings (Figure 4.9). 
A

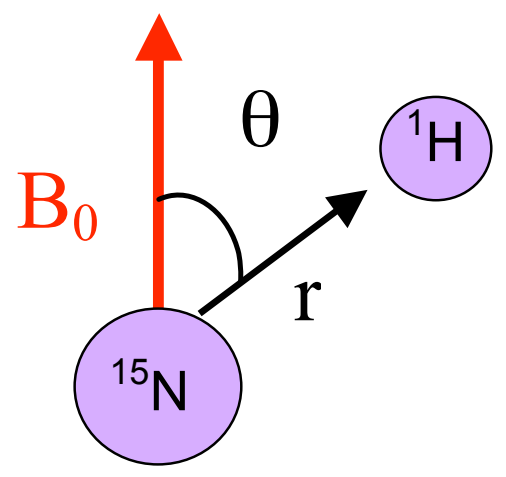

B

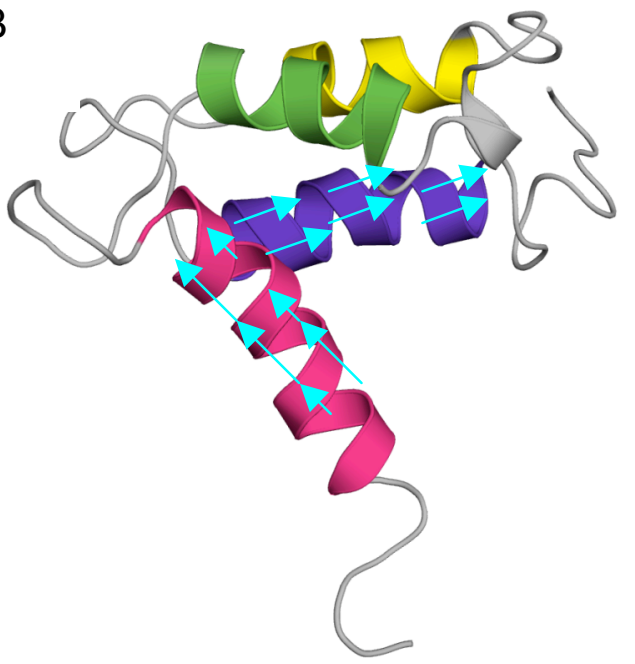

\section{Figure 4.9 Residual dipolar coupling.}

(A) Dipolar couplings between ${ }^{1} \mathrm{H}$ and ${ }^{15} \mathrm{~N}$, contain information regarding the orientation of the vector bond relative to magnetic field (Bo). $\theta$ is the angle between the internuclear vector and Bo and $r$ the distance between the two nuclei. (B) Representation of $\mathrm{H}-\mathrm{N}$ vector bonds along $\alpha$-helices regions (cyan arrows) of apo-S100A11. Within a single $\alpha$ helix ${ }^{1} \mathrm{H}-{ }^{15} \mathrm{~N}$ vector are aligned in a similar manner and are expected to show dipolar couplings similar in magnitude and of the same sign. 
Dipolar couplings between ${ }^{1} \mathrm{H}$ and ${ }^{15} \mathrm{~N}$, contain information regarding the orientation of the bond vector relative to the molecular alignment tensor as in:

$$
\mathrm{D}_{\mathrm{HN}}(\theta, \phi)=\mathrm{S}\left(\mu_{\mathrm{o}} / 4 \pi\right) \gamma_{\mathrm{H}} \gamma_{\mathrm{N}} h\left\{\mathrm{~A}_{\mathrm{a}}\left(3 \cos ^{2} \theta-1\right)+{ }^{3} / 2 \operatorname{Ar} \sin ^{2} \theta \cos 2 \phi\right\} / 4 \pi^{2} \mathrm{r}^{3}{ }_{\mathrm{HN}}
$$

where $S$ is the generalized order parameter of internal motion of the vector $\mathrm{HN}, \gamma_{\mathrm{H}} \gamma_{\mathrm{N}}$ are the gyromagnetic ratios of $\mathrm{H}$ and $\mathrm{N}, h$ is Plank's constant, $\mathrm{r}_{\mathrm{HN}}$ the distance between $\mathrm{H}$ and $\mathrm{N}, \theta$ and $\phi$ are the cylindrical coordinates that describe the orientation of the vector in the axis system, and $A_{a}$ and $A_{r}$ the axial and rhombic components of the molecular alignment tensor $[23,24]$. Amide bonds of the same $\alpha$-helix are expected to be oriented in a similar manner with respect to the magnetic field, hence any conformational changes in the helical regions would be reflected in the sign and amplitude of the couplings between the two conformations (Figure 4.9B).

To determine if a reorientation of any of the helices in apo-S100A11 ${ }^{\text {LII }}$ occurred $\mathrm{HN}$ residual dipolar coupling using two-dimensional IPAP ${ }^{1} \mathrm{H}^{15} \mathrm{~N}$ HSQC experiments were measured for both wild type and chimaeric S100A11 ${ }^{\text {LII }}$ proteins.

\subsection{Measurement of Dipolar Coupling}

Protein samples were aligned by additions of filamentous Pf1 bacteriophage [25]. Pfl phages are long, highly negatively charged rods in which a single stranded circular DNA is packaged with coat protein arranged in an $\alpha$-helical fashion. Pfl aligns spontaneously in a magnetic field and the degree of alignment can be assessed by monitoring the extent of the $\mathrm{J}$ coupling of the deuterium quadrupole splitting of the HDO 
resonance [26]. This signal appears from the exchange between bulk water and water bound to the oriented Pf1 phage molecules.

Figure 4.10 shows the ${ }^{2} \mathrm{H}$ NMR spectra of a $90 \% \mathrm{H}_{2} \mathrm{O} / 10 \% \mathrm{D}_{2} \mathrm{O}$ solution containing apo-S100A11 in $20 \mathrm{mM}$ Tris (pH 7.25), $5 \mathrm{mM}$ EDTA, $5 \mathrm{mM}$ TCEP, $50 \mathrm{mM}$ $\mathrm{KCl}$ as a function of phage concentration used to determine the best alignment conditions for the ${ }^{1} \mathrm{H}_{-}{ }^{15} \mathrm{~N}$ IPAP HSQC experiments. A higher degree of alignment was obtained using a phage concentration of $9.2 \mathrm{mg} / \mathrm{mL}(\Delta=7.5 \mathrm{~Hz})$, however this also led to signal broadening. A smaller concentration of phage was selected for the dipolar experiments (6.7 $\mathrm{mg} / \mathrm{mL}$ Pf1) where a measurable splitting was observed with only minor line broadening.

${ }^{1} \mathrm{H}-{ }^{15} \mathrm{~N}$-dipolar couplings for apo-S100A11 and apo-S100A11 ${ }^{\mathrm{LII}}$ were obtained from the change in $\mathrm{J}$ splitting measured under oriented and isotropic conditions, as illustrated in Figure 4.11 for V55 and K62 in apo-S100A11. In the absence of decoupling, the observed splitting between ${ }^{1} \mathrm{H}-{ }^{15} \mathrm{~N}$ corresponds to the sum of the scalar (J) and dipolar (D) interactions. Under isotropic conditions the dipolar components average to zero and the splitting corresponds to the scalar coupling (92-95 Hz) (Figure 4.11). Alignment of apo-S100A11 in the presence of Pf1 gives rise to the dipolar coupling contribution and the splitting corresponds to the $\mathrm{J}+\mathrm{D}$ interactions. Differences in splitting for the samples in the presence and absence of phage represent the dipolar coupling and is related to the orientation of the amide bonds with respect to the alignment frame. 
A

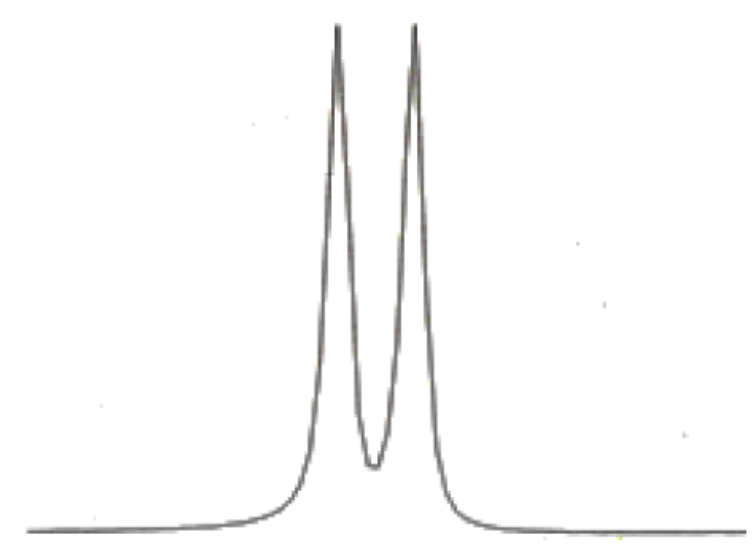

B

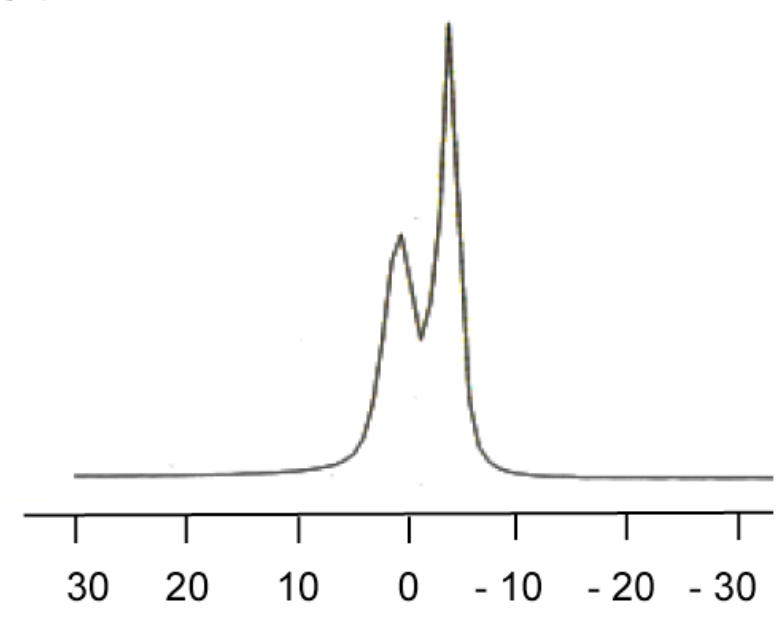

Frequency $(\mathrm{Hz})$

Figure 4.10 Magnetic alignment of Pf1 filamentous phage.

The deuterium quadrupole splitting of the water resonance was monitored by $1 \mathrm{D}{ }^{2} \mathrm{H}$ NMR as indicative of the alignment of the Pf1 phage in the apo-S100A11 NMR sample. Two different protein/Pf1 conditions were tested (A) $0.75 \mathrm{mM}$ and $6.7 \mathrm{mg} / \mathrm{mL}$ (B) 1.0 $\mathrm{mM}$ and $9.2 \mathrm{mg} / \mathrm{mL}$. Splitting values were 7.5 and 5.4 respectively. Apo-S100A11 at a concentration of $0.75 \mathrm{mM}$ in the presence of $6.7 \mathrm{mg} / \mathrm{mL}$ of phage arise the most signal in the ${ }^{1} \mathrm{H}-{ }^{15} \mathrm{~N}$ IPAP-HSQC experiments with narrowest linewidths. 

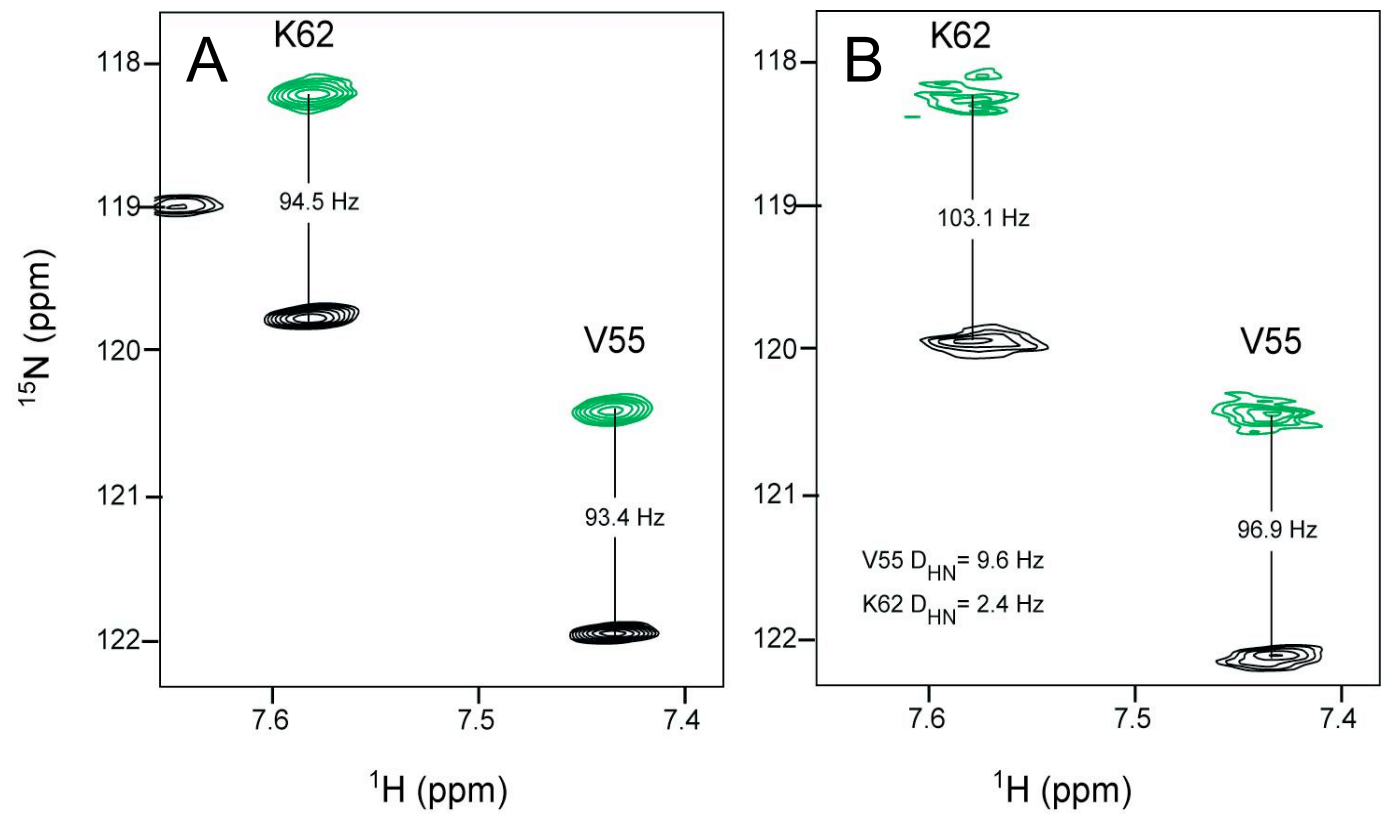

Figure 4.11 Examples of the changes in ${ }^{1} \mathrm{H}-{ }^{15} \mathrm{~N}$ heteronuclear splittings of apoS100A11.

Representative regions of the $2 \mathrm{D}{ }^{1} \mathrm{H}^{-15} \mathrm{~N}$ IPAP-HSQC NMR spectra of apo-S100A11 used to measure residual dipolar couplings. The region illustrates the splitting of V55 and K62. Dipolar couplings were determined by subtracting the splitting in the presence (B) and absence (A) of aligning Pf1 medium. 
the orientation of the $\mathrm{HN}$ bonds in the partially aligned apo-S100A11 in the magnetic field. The figure shows a clear pattern of couplings within a single helix, for helices I, II and IV, where the dipolar couplings have the same sign and oscillating amplitudes. Since the amide bonds are oriented along the axis of the alpha helix, it would be expected that $\mathrm{HN}$ vectors form the same $\alpha$-helix are oriented in a similar manner in the magnetic field. Interestingly, helix III seems to break the pattern, with dipolar couplings that are both positive and negative and of different magnitudes. Structural studies have shown that in the absence of calcium helix III is loosely packed with amide protons that exchange up to three orders of magnitude faster than those in other helices $[27,28]$. In a similar mode, residual dipolar couplings for apo-S100A11 could be reflecting the average of a range of conformations that helix III can occupy or poor definitions in the helix arising to a large variation in the couplings. The plot also highlights the negative sign of the couplings for helices I and IV, as a result of the coplanar orientation of the two helices, in agreement with residual dipolar couplings studies previously reported for S100B [28, 29].

\subsection{Refinement of apo-S100A11 structure}

Comparison of residual dipolar couplings measured experimentally for apoS100A11 were compared to those calculated from the NMR structure (PDB 1NSH using the most representative model 12) shows a low correlation between the two $(\mathrm{RMS}=6.56$ $\mathrm{Q}=0.736, \operatorname{Rdip}=0.780)($ Figure 4.13$) . \quad$ The NMR structure of apo-S100A11 was refined using ${ }^{1} \mathrm{H}^{-15} \mathrm{~N}$ residual dipolar couplings measured for the helical regions, in order to have a good reference structure to compare with the residual dipolar couplings from the chimaeric apo-S100A11 ${ }^{\text {LI }}$ protein. 


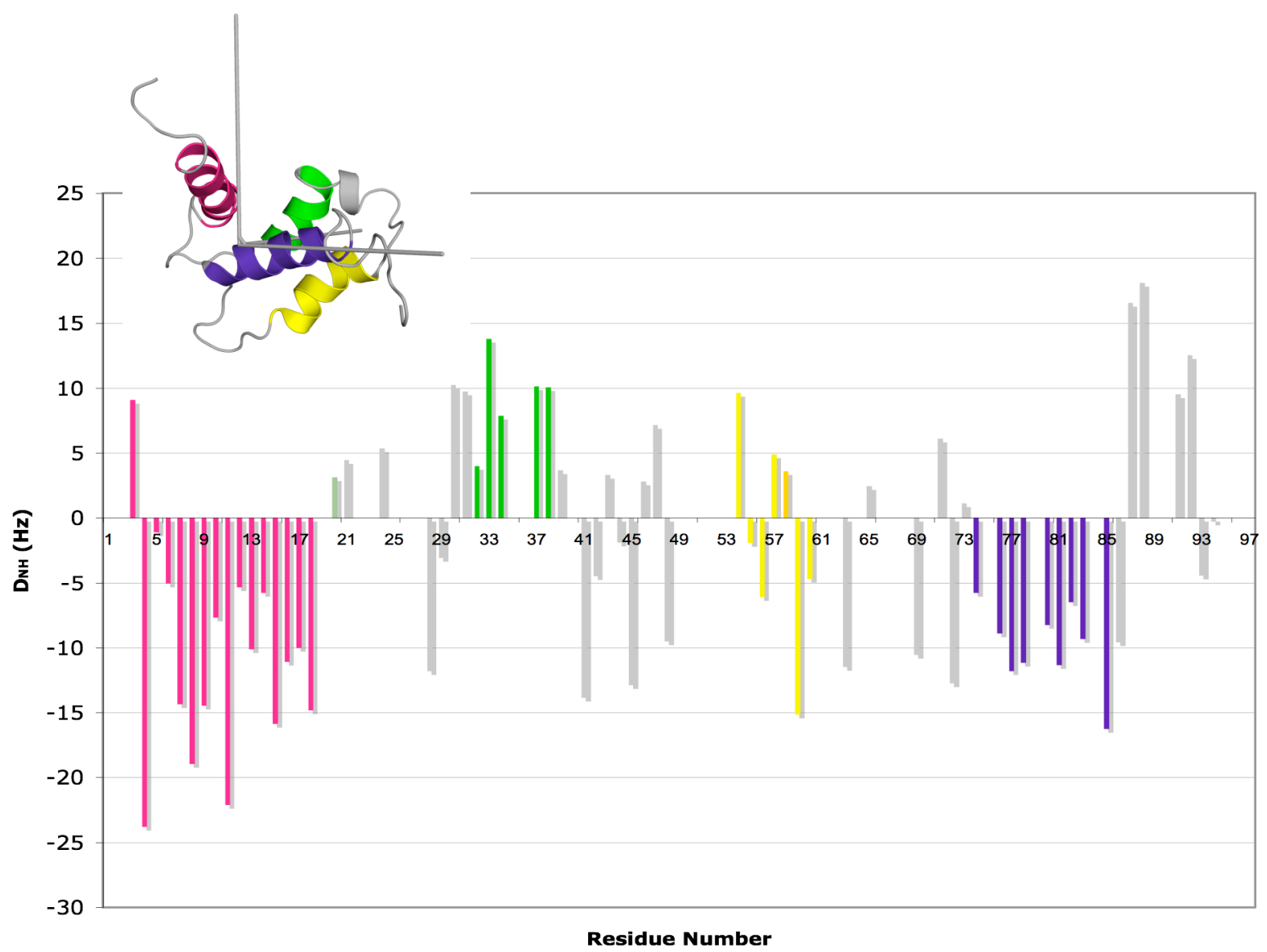

\section{Figure 4.12 Experimental ${ }^{1} \mathrm{H}^{15} \mathrm{~N}$ dipolar coupling for apo-S100A11.}

Dipolar couplings are plotted as magnitude vs. residue number. Helical regions are coloured as follows helix I (pink), helix II (green), helix III (yellow) and helix IV (blue). The $\mathrm{D}_{\mathrm{HN}}$ values within a single $\alpha$-helix have oscillating amplitudes and same sign, except for helix III (yellow). Only residues that exhibited clearly resolved HN correlations were measured. The three-dimensional structure of apo-S100A11 (protomer) in its principal frame is shown above the plot. Helix I (pink) and IV are coplanar and orient in a similar manner with respect to the magnetic field with dipolar couplings of the same sign. 

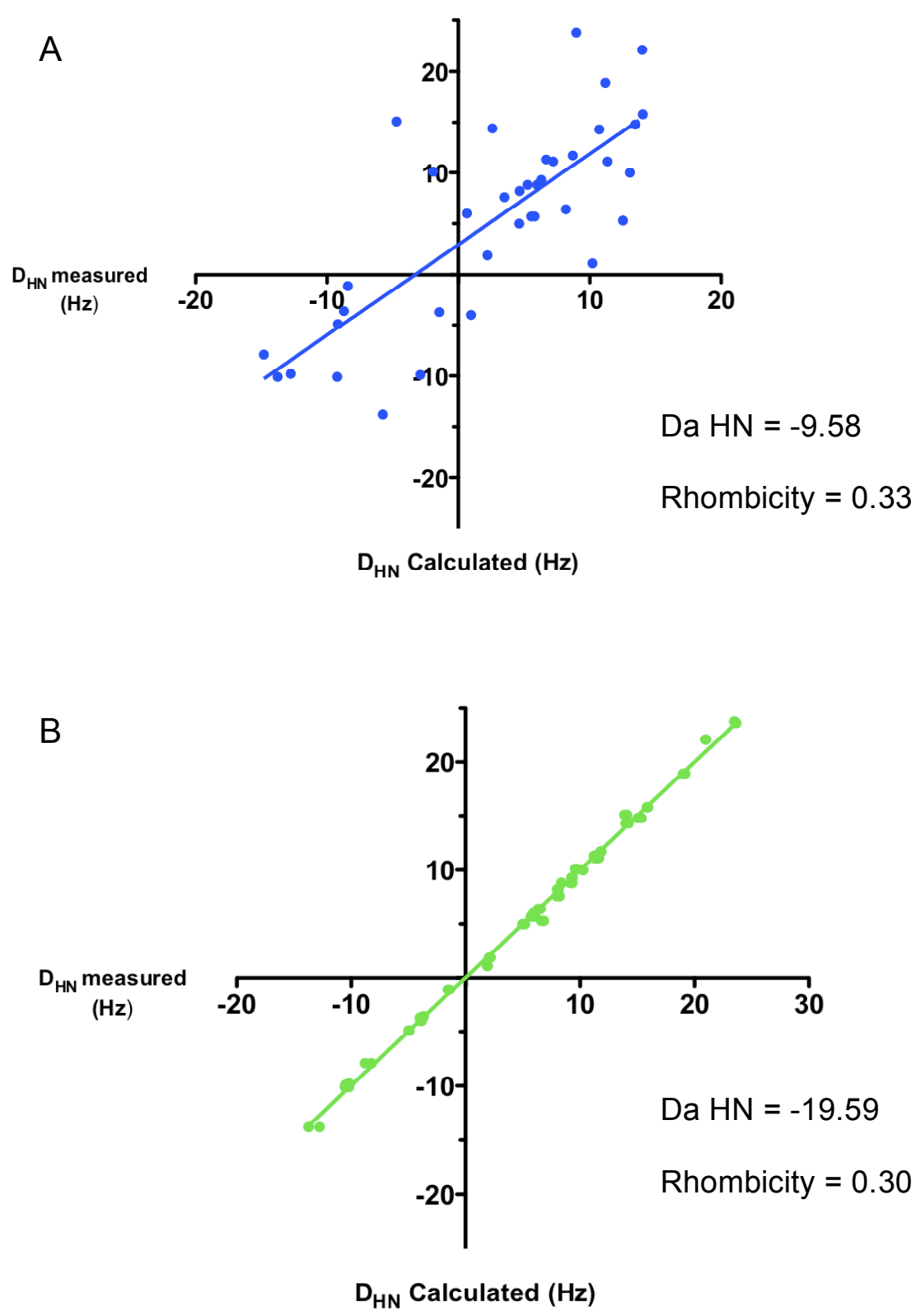

Figure 4.13 Apo-S100A11 structure refinement using RDCs.

Plot of experimental ${ }^{1} \mathrm{H}_{-}{ }^{15} \mathrm{~N}$ RDCs vs calculated ${ }^{1} \mathrm{H}_{-}{ }^{15} \mathrm{~N}$ RDC restraitns for the structure of apo-S100A11 prior to (A) and after (B) the refinement. The refinement resulted in an improvement in the correlation of the couplings as reflected in a lower Q value for the refined structure ( 0.27 compared to 0.736 in the initial structure). Axial and rhombic components of the alignment tensor are noted as $\mathrm{Da} H \mathrm{HN}$ and $\mathrm{R}$ respectively. The precision of the measured RDCs is $\sim 1 \mathrm{~Hz}$. 
Geometric information from the dipolar couplings of apo-S100A11 were used during a low temperature simulated annealing (DYNAMO) protocol, which minimizes the difference between the measured dipolar couplings and those calculated form the structure. The marked improvement of the correlation can bee seen in Figure 4.13. The Q value was now 0.48 although the difference between the refined and original structures of apo-S100A11 was only $0.20 \AA$, which it is lower than that reported for the family of NMR structures $(0.51 \AA)$, indicating the refinement had little impact on the overall fold of the protein.

\subsection{Substitutions in loop II causes reorientation of helices in S100A11}

In an analogous way as described for apo-S100A11 the residual dipolar couplings for apo-S100A11 ${ }^{\mathrm{LII}}$ were measured from the differences in the splitting in partially aligned and isotropic samples (Figure 4.14).

The dipolar couplings values of $\mathrm{D}_{\mathrm{HN}}$ measured for the chimaeric protein are plotted in Figure 4.15. It is worth noting that in S100A1 $1^{\mathrm{LII}}$ the dipolar couplings in helix III are more homogeneous and have the same sign. It has been shown that the calciuminduced conformational change in S100 proteins has an impact in the dynamics of the protein, particularly in helix III and the second calcium-binding site. Three-dimensional structures of $\mathrm{Ca}^{2+}$-bound $\mathrm{S} 100$ proteins show that helix III occupies a narrower range of conformations, unlike in the apo-state, implying an increased rigidity of the helix as a result of the binding of calcium and conformational change [29-35].

The measured dipolar couplings for S100A $11^{\mathrm{LII}}$ seem to suggest that a change in the orientation of helix III occurred, compatible with the open form of S100A11. 

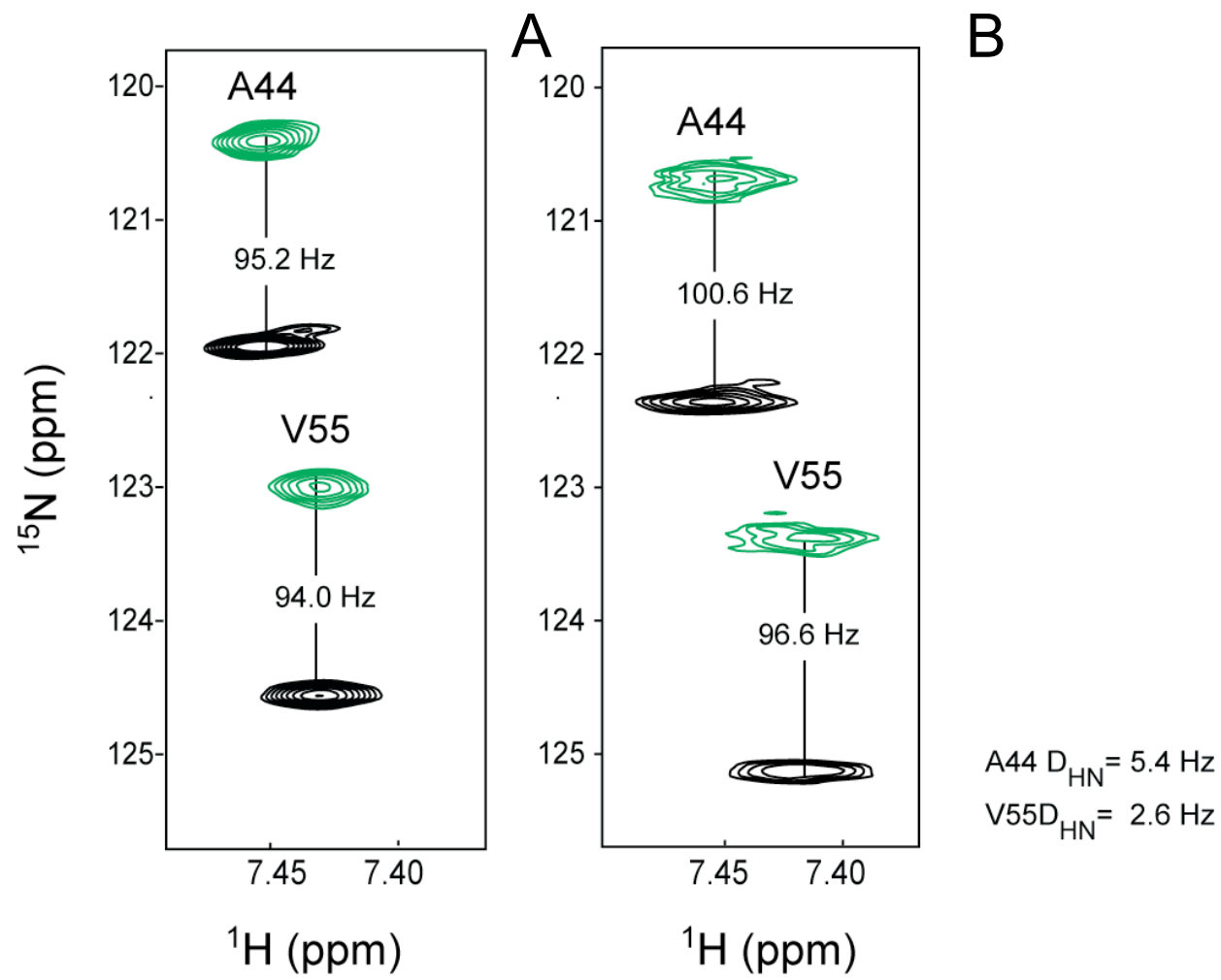

Figure 4.14 Measurement of residual dipolar couplings for apo-S100A11 ${ }^{\text {LII }}$.

A portion of the $2 \mathrm{D}{ }^{1} \mathrm{H}^{-15} \mathrm{~N}$ IPAP-HSQC spectra collected for apo-S100A1 ${ }^{\text {LII }}(1.0 \mathrm{mM})$ with (A) no phage and (B) with $8.3 \mathrm{mg} / \mathrm{mL}$ Pfl phage. This region of the spectrum shows the effect of the aligning medium Pf1 for the amide bonds of A44 and V55. Dipolar couplings are determined as the difference in splitting in anisotropic (B) and isotropic conditions (A). 


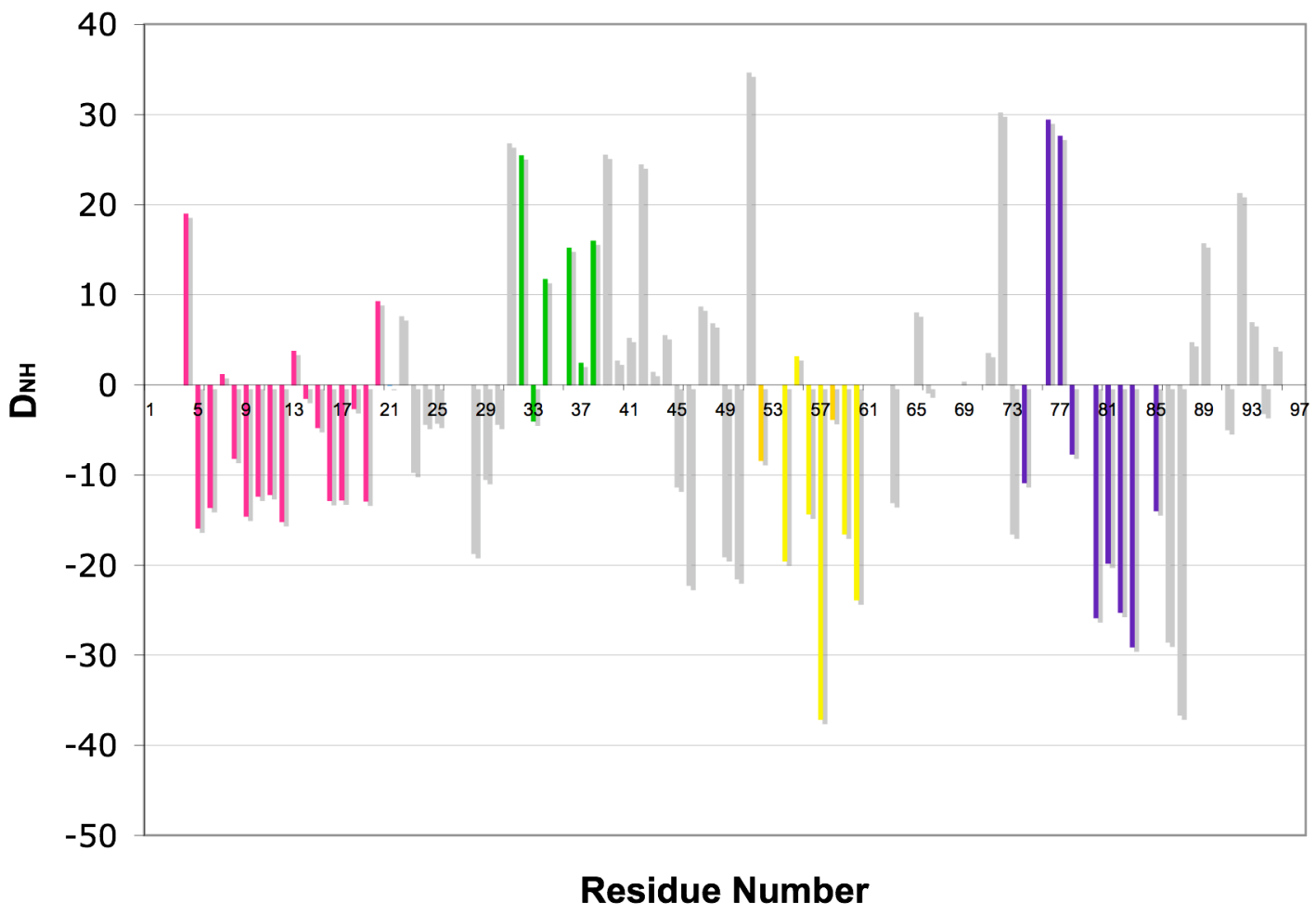

Figure 4.15 Experimental ${ }^{1} \mathrm{H}-{ }^{15} \mathrm{~N}$ dipolar couplings for apo-S100A11 ${ }^{\mathrm{LII}}$.

Dipolar couplings are plotted as magnitude vs. residue number. Helical regions are coloured as follows helix I (pink), helix II (green), helix III (yellow) and helix IV (blue). Only residues that exhibited clearly resolved HN correlations were measured. 
Alternatively, an increase in the population of the open state could also account for the differences in helix III compared to that of apo-S100A11. Further analyses and fittings of the dipolar coupling to the apo- and $\mathrm{Ca}^{2+}-\mathrm{S} 100 \mathrm{~A} 11$ structures will be discussed to test this hypothesis.

The measured $\mathrm{D}_{\mathrm{HN}}$ for apo-S100A11 and apo-S100A11 ${ }^{\mathrm{LII}}$ were mostly similar along helices I and the C-termini of helix IV, while large differences were observed all throughout helix II, C-terminal region of helix III and N-terminal of helix IV. This suggests that substitutions in calcium binding site II caused changes in the orientations of helices II, III and N-terminal of helix IV (Figure 4.16)

The experimental dipolar couplings for the $\mathrm{Ca}^{2+}-\mathrm{S} 100 \mathrm{~A} 11$ could not be measured using Pfl phage as the alignment media since NMR samples of ${ }^{15} \mathrm{~N}$-labeled $\mathrm{Ca}^{2+}$ S100A11 in the presence of the alignment molecule resulted in aggregation or precipitation of the protein. It is possible that the phage, which is negatively charged, is interacting with calcium ions or other charged residues in the protein leading to its precipitation. Precipitation was also experienced during alignment trials of apoS100A11 ${ }^{\text {LII }}$, suggesting structural differences with respect to apo-S100A11, since both dipolar coupling were measured in excess of EDTA and apo-S100A11 did not exhibit any signs of aggregation. It is also possible that increased electrostatic interactions due to conformational differences between apo-S100A11 and S100A11 ${ }^{\mathrm{LII}}$ or $\mathrm{Ca}^{2+}-\mathrm{S} 100 \mathrm{~A} 11$ of the protein are causing the protein to aggregate. For the purpose of comparison, the calculated dipolar couplings for $\mathrm{Ca}^{2+}-\mathrm{S} 100 \mathrm{~A} 11$ derived from the three-dimensional structure were used. 


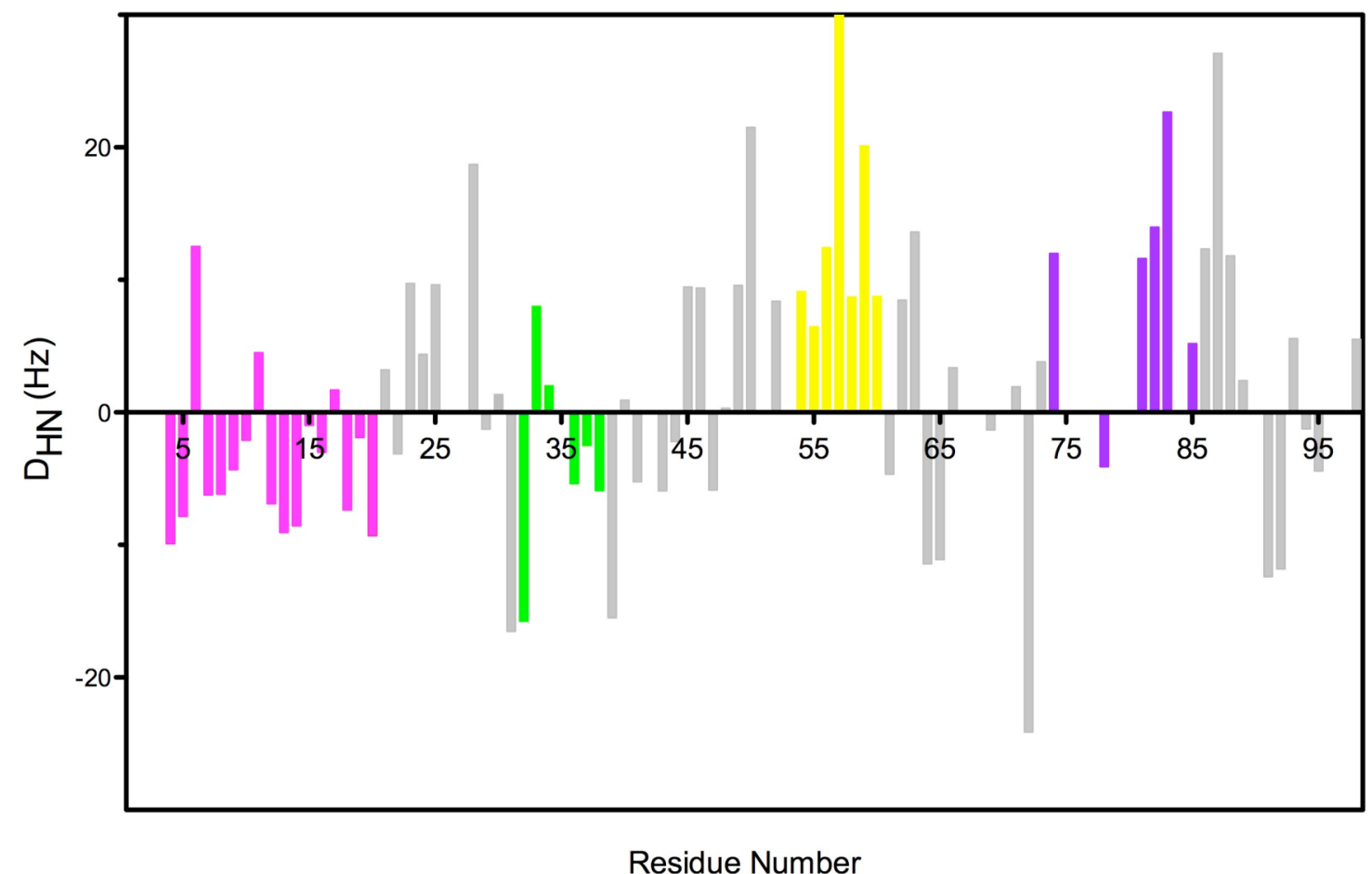

Figure 4.16 Structural effects of substitution in loop II in apo-S100A11 as evidenced by RDCs.

Dipolar couplings are plotted as the difference in magnitude between couplings of S100A11 ${ }^{\mathrm{LII}}$ and apo-S100A11 vs. residue number. Helical regions are coloured as follows helix I (pink), helix II (green), helix III (yellow) and helix IV (blue). The $\mathrm{D}_{\mathrm{HN}}$ values for apo-S100A11 and apo-S100A11 ${ }^{\mathrm{LII}}$ are distinct, particularly along helix III, suggesting conformational differences in the chimaeric protein as a result of modified calcium-binding loop 2. 
Figure 4.17 represents the correlation of experimental residual dipolar couplings of S100A11 ${ }^{\mathrm{LII}}$ with calculated dipolar couplings of the refined apo-S100A11 structure and $\mathrm{Ca}^{2+}-\mathrm{S} 100 \mathrm{~A} 11$ and the results are summarized in Table 4.1. Q and $\mathrm{R}_{\text {dip }}$ are qualitative measures of the agreement with a lower value indicative of better correlation.

The correlation of the $\mathrm{D}_{\mathrm{HN}}$ for apo-S100A11 ${ }^{\mathrm{LII}}$ with apo-S100A11 and $\mathrm{Ca}^{2+}$ S100A11 are poor, with Q values with apo-S100A11 higher than correlation of apoS100A11 and $\mathrm{Ca}^{2+} \mathrm{S} 100 \mathrm{~A} 11$, and slightly lower when correlated with $\mathrm{Ca}^{2+}-\mathrm{S} 100 \mathrm{~A} 11$. The degree of quality in terms of linear regression are consistent with correlation coefficients and quality factors that also show the low agreement of the RDCs for the structures compared. Overall the residual dipolar coupling results show that the structure of $\mathrm{S} 100 \mathrm{~A} 11^{\mathrm{LII}}$ has been altered as a result of the substitutions introduced in site II. It is interesting that the residual dipolar couplings of $\mathrm{S} 100 \mathrm{~A} 11^{\mathrm{LII}}$, do not agree with the refined couplings for apo-S100A11 nor for $\mathrm{Ca}^{2+}-\mathrm{S} 100 \mathrm{~A} 11$, indicating that the orientation of the helices in S100A1 $1^{\mathrm{LII}}$ are not identical to either structure.

Overall, structural studies using NMR spectroscopy suggest that the chimaeric $\mathrm{S} 100 \mathrm{~A} 11^{\mathrm{LII}}$ protein displays intermediate properties between apo-S100A11 and $\mathrm{Ca}^{2+}$ S100A11 and does not fit either the closed or the open structure of S100A11. This could result from S100A11 ${ }^{\mathrm{LII}}$ adopting a different structure from those either apo-S100A11 or Ca2+-S100A11, or most likely, considering the equilibrium that exist between open/closed states in the EF-hand proteins, from fast exchange between multiple conformations. 

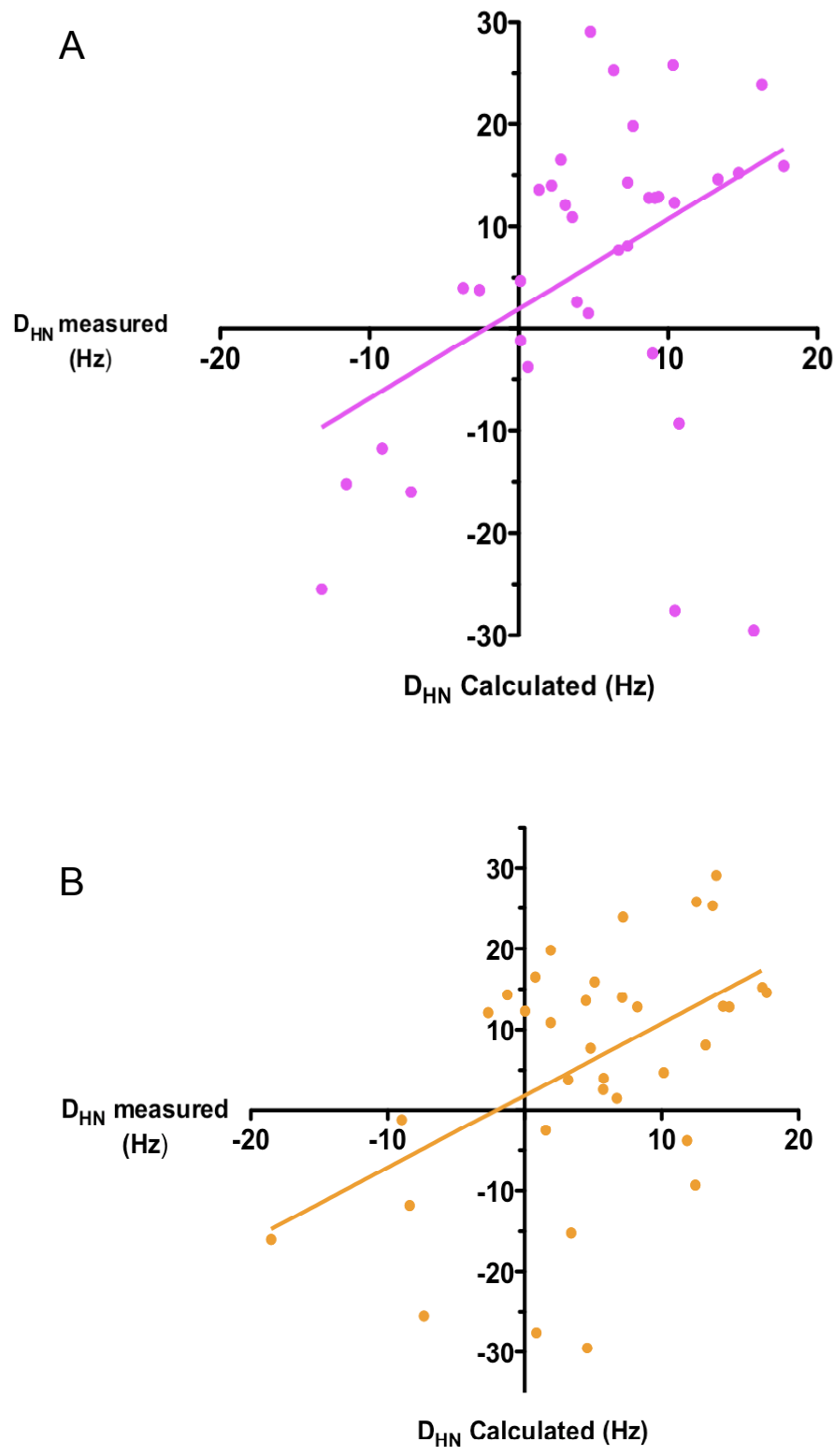

Figure 4.17 Correlation between measured and calculated ${ }^{1} \mathrm{H}-{ }^{15} \mathrm{~N}$ dipolar couplings for S100A11 and S100A11 ${ }^{\mathrm{LII}}$.

Correlation of backbone ${ }^{1} \mathrm{H}-{ }^{15} \mathrm{~N}$ dipolar couplings for apo-S100A11 ${ }^{\mathrm{LII}}$ with the refined apo-S100A11 (A) and $\mathrm{Ca}^{2+}-\mathrm{S} 100 \mathrm{~A} 11$ (B) structures. Comparison involves only the alpha helical regions. The S100A1 $1^{\mathrm{LII}}$ couplings fit poorly to both apo- and $\mathrm{Ca}^{2+}$-bound RDCs of S100A11 with RMS $\sim 14 \mathrm{~Hz}$ and high Q values close to 1 . 
Table 4.1. Correlation of experimental RDCs of S100A11 ${ }^{\mathrm{LII}}$ and calculated S100A11.

\begin{tabular}{|c|c|c|c|c|c|c|}
\hline Structure & $\mathbf{D}_{\mathrm{HN}}$ & ${ }^{\mathbf{a}} \mathbf{R M S}$ & $\mathrm{b}, \mathbf{c}^{\mathrm{Q}} \mathbf{Q}$ & ${ }^{\mathbf{b}} \mathbf{R}$ dip & ${ }^{\mathrm{d}}$ Slope & ${ }^{d} \mathbf{r}^{2}$ \\
\hline $\begin{array}{l}\text { apo-S100A11 } \\
\text { (refined) } \\
\mathrm{Ca}^{2+}-\mathrm{S} 100 \mathrm{~A} 11\end{array}$ & $\begin{array}{l}\text { apo-S100A11 } \\
\text { apo-S100A } 11^{\text {LII }}\end{array}$ & 14.19 & 0.86 & 0.421 & 0.88 & 0.17 \\
\hline $\mathrm{Ca}^{2+}-\mathrm{S} 100 \mathrm{~A} 11$ & apo-S100A11 & $\begin{array}{r}13.97 \\
6.38\end{array}$ & 0.63 & 0.45 & 0.89 & 0.20 \\
\hline
\end{tabular}

${ }^{a}$ Root-mean-square deviation between input and calculated RDCs

${ }^{\mathrm{b}}$ Q-factor and Rdip factor are each calculated using Dynamo program

Rdip $=$ Pearson's linear correlation coefficient between input and calculated RDCs (range: [-1, 1])

${ }^{\mathrm{c}} \mathrm{Q}$ factor is computed as $\sqrt{ } \sum\left(\mathrm{D}_{\mathrm{HN}}\right.$ meas $-\mathrm{D}_{\mathrm{HN}}$ calc $\left.) / \mathrm{N} *\left(\mathrm{Da}^{2} *\left(4+3 \mathrm{Rh}^{2}\right) / 5\right)\right]$

Where DHN meas $=$ DHN measured; DHN calcu $=$ DHN calculated; $\mathrm{N}=$ number of measured couplings; $\mathrm{Rh}=$ rombicity

${ }^{d}$ parameters of linear regression 


\subsection{Conclusion}

Seven chimaeric proteins where regions from calcium-binding sites I and II, and helices II-IV in S100A11 were replaced with the corresponding regions of S100A10 were characterized in terms of increased hydrophobic surface area, calcium-insensitive target binding and structural effects. The chimaeric proteins having substitutions in calciumbinding site II displayed increased hydrophobic surface exposure as assessed by ANS fluorescence and phenyl Sepharose binding in the absence of calcium. This response is similar to that observed for $\mathrm{Ca}^{2+}-\mathrm{S} 100 \mathrm{~A} 11$ and calcium-free S100A10. Further, this substitution resulted in calcium-insensitive binding to annexin A2. These results indicate that residues within site II contribute significatively to the conformation of S100A10 and presentation of its target-binding site. In contrast, S100A11 chimaeric proteins with helical substitutions displayed poorer hydrophobic surface exposure and consequently, unobservable annexin A2 binding. In addition, NMR studies showed significant structural differences of S100A11 ${ }^{\text {LII }}$ compared with S100A11 particularly in the orientation of helices II and III in partially aligned samples respect to the magnetic field.

In summary, the results suggest that residues in site II are important contributors towards the open state in S100A10. Further refinement of these chimaeric proteins should allow the design of an S100A11 protein that favours the open conformation and allow its interaction with target proteins in a calcium-independent manner. 


\subsection{References}

1 Tjandra, N., Kuboniwa, H., Ren, H. and Bax, A. (1995) Rotational dynamics of calcium-free calmodulin studied by $15 \mathrm{~N}-\mathrm{NMR}$ relaxation measurements. Eur J Biochem. 230, 1014-1024

2 Malmendal, A., Evenas, J., Forsen, S. and Akke, M. (1999) Structural dynamics in the C-terminal domain of calmodulin at low calcium levels. J Mol Biol. 293, 883-899

3 Evenas, J., Malmendal, A. and Akke, M. (2001) Dynamics of the transition between open and closed conformations in a calmodulin C-terminal domain mutant. Structure. 9, 185-195

4 Evenas, J., Forsen, S., Malmendal, A. and Akke, M. (1999) Backbone dynamics and energetics of a calmodulin domain mutant exchanging between closed and open conformations. J Mol Biol. 289, 603-617

5 Ababou, A., Shenvi, R. A. and Desjarlais, J. R. (2001) Long-range effects on calcium binding and conformational change in the N-domain of calmodulin. Biochemistry. 40, 12719-12726

6 Bunick, C. G., Nelson, M. R., Mangahas, S., Hunter, M. J., Sheehan, J. H., Mizoue, L. S., Bunick, G. J. and Chazin, W. J. (2004) Designing sequence to control protein function in an EF-hand protein. J Am Chem Soc. 126, 5990-5998

7 Nelson, M. R., Thulin, E., Fagan, P. A., Forsen, S. and Chazin, W. J. (2002) The EFhand domain: a globally cooperative structural unit. Protein Sci. 11, 198-205

8 Rety, S., Sopkova, J., Renouard, M., Osterloh, D., Gerke, V., Tabaries, S., RussoMarie, F. and Lewit-Bentley, A. (1999) The crystal structure of a complex of p11 with the annexin II N-terminal peptide. Nat Struct Biol. 6, 89-95

9 Rasband, W. S. (1997-2008) ImageJ, U.S. National Institutes of Health, Bethesda, Maryland, USA, http://rsb.info.nih.gov/ij/,

10 Kay, L. E., Ikura, M., Tschudin, R. and Bax, A. (1990) Three-dimensional tripleresonance NMR spectroscopy of isotopically enriched proteins. J. Magn. Reson. 89, 496-514

11 Wittekind, M. and Mueller, L. (1993) HNCACB, A high sensitivity 3D NMR experiment to correlate amide proton and nitrogen resonances with the $\{$ alpha $\}$ carbon and ß-carbon resonances in proteins. J. Magn. Reson. Series B. 101, 171-180

12 Grzesiek, S. and Bax, A. (1992) Correlating backbone amide and side chain resonances in larger proteins by multiple relayed triple resonance NMR. J. Am. Chem. Soc. 114, 6291-6293

13 Delaglio, F., Grzesiek, S., Vuister, G. W., Zhu, G., Pfeifer, J. and Bax, A. (1995) NMRPipe: A multidimensional spectral processing system based on UNIX pipes. J. Biomol. NMR. 6, 277-293

14 Johnson, B. A. and Belvins, R. A. (1994) NMRView: A computer program for the visualization and analysis of NMR data. J. Biomol. NMR. 4, 603-614

15 Ottiger, M., Delaglio, F. and Bax, A. (1998) Measurement of J and dipolar couplings from simplified two-dimensional NMR spectra. J Magn Reson. 131, 373-378

16 Dempsey, A. C., Walsh, M. P. and Shaw, G. S. (2003) Unmasking the annexin I interaction from the structure of Apo-S100A11. Structure (Camb). 11, 887-897 
17 Baudier, J., Labourdette, G. and Gerard, D. (1985) Rat brain S100b protein: purification, characterization, and ion binding properties. A comparison with bovine S100b protein. J Neurochem. 44, 76-84

18 Steiner, R. F. and Norris, L. (1987) Fluorescence dynamics studies of troponin C. Biopolymers. 26, 1189-1204

19 Steiner, R. F. and Sternberg, H. (1982) Properties of the complexes formed by 1anilinonaphthalene-8-sulfonate with phosphorylase kinase and calmodulin. Biopolymers. 21, 1411-1425

20 Semisotnov, G. V., Rodionova, N. A., Razgulyaev, O. I., Uversky, V. N., Gripas, A. F. and Gilmanshin, R. I. (1991) Study of the "molten globule" intermediate state in protein folding by a hydrophobic fluorescent probe. Biopolymers. 31, 119-128

21 Shaw, G. S., Marlatt, N. M., Ferguson, P. L., Barber, K. R. and Bottomley, S. P. (2008) Identification of a dimeric intermediate in the unfolding pathway for the calcium-binding protein S100B. J Mol Biol. 382, 1075-1088

22 Rintala-Dempsey, A. C., Santamaria-Kisiel, L., Liao, Y., Lajoie, G. and Shaw, G. S. (2006) Insights into S100 target specificity examined by a new interaction between S100A11 and annexin A2. Biochemistry. 45, 14695-14705

23 Tjandra, N. and Bax, A. (1997) Direct measurement of distances and angles in biomolecules by NMR in a dilute liquid crystalline medium. Science. 278, 1111-1114

24 Tjandra, N., Omichinski, J. G., Gronenborn, A. M., Clore, G. M. and Bax, A. (1997) Use of dipolar $1 \mathrm{H}-15 \mathrm{~N}$ and $1 \mathrm{H}-13 \mathrm{C}$ couplings in the structure determination of magnetically oriented macromolecules in solution. Nat Struct Biol. 4, 732-738

25 Hansen, M. R., Mueller, L. and Pardi, A. (1998) Tunable alignment of macromolecules by filamentous phage yields dipolar coupling interactions. Nat Struct Biol. 5, 1065-1074

26 de Alba, E. and Tjandra, N. (2004) Residual dipolar couplings in protein structure determination. Methods Mol Biol. 278, 89-106

27 Marlatt, N. M. and Shaw, G. S. (2007) Amide exchange shows calcium-induced conformational changes are transmitted to the dimer interface of S100B. Biochemistry. 46, 7478-7487

28 Malik, S., Revington, M., Smith, S. P. and Shaw, G. S. (2008) Analysis of the structure of human apo-S100B at low temperature indicates a unimodal conformational distribution is adopted by calcium-free S100 proteins. Proteins. 73, $28-42$

29 Drohat, A. C., Tjandra, N., Baldisseri, D. M. and Weber, D. J. (1999) The use of dipolar couplings for determining the solution structure of rat apo-S100B(betabeta). Protein Sci. 8, 800-809

30 Maler, L., Potts, B. C. and Chazin, W. J. (1999) High resolution solution structure of apo calcyclin and structural variations in the S100 family of calcium-binding proteins. J Biomol NMR. 13, 233-247

31 Drohat, A. C., Baldisseri, D. M., Rustandi, R. R. and Weber, D. J. (1998) Solution structure of calcium-bound rat $\mathrm{S} 100 \mathrm{~B}$ (betabeta) as determined by nuclear magnetic resonance spectroscopy. Biochemistry. 37, 2729-2740 
32 Smith, S. P. and Shaw, G. S. (1998) A novel calcium-sensitive switch revealed by the structure of human S100B in the calcium-bound form. Structure. 6, 211-222

33 Kilby, P. M., Van Eldik, L. J. and Roberts, G. C. (1996) The solution structure of the bovine S100B protein dimer in the calcium-free state. Structure. 4, 1041-1052

34 Sastry, M., Ketchem, R. R., Crescenzi, O., Weber, C., Lubienski, M. J., Hidaka, H. and Chazin, W. J. (1998) The three-dimensional structure of $\mathrm{Ca}(2+)$-bound calcyclin: implications for $\mathrm{Ca}(2+)$-signal transduction by $\mathrm{S} 100$ proteins. Structure. 6, 223-231

35 Otterbein, L. R., Kordowska, J., Witte-Hoffmann, C., Wang, C. L. and Dominguez, R. (2002) Crystal structures of S100A6 in the $\mathrm{Ca}(2+)$-free and $\mathrm{Ca}(2+)$-bound states: the calcium sensor mechanism of $\mathrm{S} 100$ proteins revealed at atomic resolution. Structure. 10, 557-56 


\section{Chapter 5}

\section{ENERGETICS OF S100-PEPTIDE INTERACTIONS ${ }^{1}$}

\subsection{Introduction}

NMR spectroscopy and X-ray crystallography have been used to determine threedimensional structures of several S100 proteins in complex with peptides corresponding to binding regions of diverse target molecules. These include S100A10 in complex with the N-terminus of the phospholipid-binding protein annexin $\mathrm{A} 2[1], \mathrm{Ca}^{2+}-\mathrm{S} 100 \mathrm{~A} 11\left(\mathrm{Ca}^{2+}-\right.$ S100A11) bound to the N-terminal portion of annexin A1 [2]; $\mathrm{Ca}^{2+}-\mathrm{S} 100 \mathrm{~B}$ (calciumbound S100B) interacting with peptides derived from the C-terminal region of p53 [3], the actin-capping protein CapZ (TRTK12) [4], and the N-terminal regulatory domain from Ndr Kinase [5]; calcium-bound S100A1 $\left(\mathrm{Ca}^{2+}-\mathrm{S} 100 \mathrm{~A} 1\right)$ in complex with segments from the ryanodine receptor (Ryr) [6] and CapZ; and $\mathrm{Ca}^{2+} \mathrm{S} 100 \mathrm{~A} 6$ in complex with the C-terminal portion from Siah-1 interacting protein (SIP) [7]. Structural and sequence comparisons show that S100 proteins have similar patterns of hydrophobic residues on their binding surfaces and selectivity and specificity is conferred in part by differences in side chains at the binding surfaces as well as in the less conserved regions of the S100 proteins (linker and helix IV). In general the detailed structures have shown how some S100 proteins interact with a target protein. However much less is known about the forces driving these biomolecular associations in solution.

\footnotetext{
${ }^{1}$ Parts of this chapter have been extracted from a published manuscript: Rintala-Dempsey AC, Santamaria-Kisiel L, Liao Y, Lajoie G, Shaw GS. (2006). Insights into S100 target specificity examined by a new interaction between S100A11 and annexin A2. Biochemistry 45(49): 14695705.
} 
During S100-target complex formation a series of energetically costly events occur including the burial of solvent-exposed surfaces on both the S100 protein and the peptide and formation and breaking of bonds due to new side chain interactions and solvation effects. To gain insights into the specificity and energy contributions that stabilize S100-target complex formation, the crossreactivity and thermodynamics of the interactions of S100A11 and S100A10 with members of the annexin family are described in this chapter. Peptide array assays were used to investigate the interaction of annexin A2 with S100A11. Given the sequence and structural similarity between S100A11 and S100A10 it is possible that they can form complexes with the same target proteins. Further, the thermodynamics contributions of the interactions are studied using Isothermal Titration Calorimetry (ITC).

\subsection{Materials and Methods}

\subsubsection{Protein Expression and Purification:}

The expression and purification of rabbit S100A11 and rabbit S100A10 followed those described in Chapter 3. GST-tagged S100A11, used for peptide arrays, was overexpressed in Escherichia coli strain BL21(DE3). Cells were grown in 1L of 2xYT medium to $\mathrm{OD}_{595}$ of 0.7 and expressed using $0.4 \mathrm{mM}$ IPTG. The lysed cells were centrifuged at $38000 \mathrm{rpm}$ and purified by hydrophobic interaction chromatography as outlined in Chapter 2. 


\subsubsection{Synthesis of Target Peptides}

Annexin A1 (AMVSEFLKQAWFIDNEERR) and annexin A2 (STVHEILSKLSLEGD) peptides were synthesized using solid-phase peptide synthesis employing the F-moc chemistry strategy. The N-termini of both peptides was acetylated and a C-terminal NEERR sequence was added to annexin A1 to improve solubility. All peptides were purified by $\mathrm{C}_{18}$ reversed-phase HPLC using 0-100\% acetonitrile gradient in the presence of $0.1 \%$ trifluoroacetic acid, lyophilized and their molecular weights verified by electrospray mass spectroscopy. The calculated molecular masses for acetylated annexin A1 and annexin A2 were 2411.68 and 1669.81 Da respectively, which corresponds to the observed values 2411.49 and 1668.86 Da. All other peptides were commercially synthesized. The purity of all peptides utilized in the studies was $>95 \%$ as judged by analytical high-pressure liquid chromatography and used without further purification.

\subsubsection{Peptide Array Assays}

Peptides corresponding to the sequences of annexin A1 (AMVSEFLKQAWFID) and annexin A2 (STVHEILSKLSLEG), with an alanine scan of each amino acid, and their reverse sequences were synthesized on a cellulose membrane using an Auto-Spot Robot ASP 222 (Abimed) based on F-moc chemistry [8]. The N-terminal end of both annexin peptides was acetylated using acetic anhydride and the $\mathrm{C}$-terminal portion was covalently attached to an alanine anchor group. Figure 5.1 contains the sequences synthesized on the membrane. 


$\begin{array}{lc} & \text { AnnexinA1 } \\ \text { WT } & \text { AMVSEFLKQAWFI D } \\ \text { WT } & \text { AMVSEFLKQAWFI D } \\ \text { WT } & \text { AMVSEFLKQAWFI D } \\ 2 & \text { AAVSEFLKQAWFI D } \\ 3 & \text { AMASEFLKQAWFI D } \\ 4 & \text { AMVAEFLKQAWFI D } \\ 5 & \text { AMVSAFLKQAWFI D } \\ 6 & \text { AMVSEALKQAWFI D } \\ 7 & \text { AMVSEFAKQAWFI D } \\ 8 & \text { AMVSEFLAQAWFI D } \\ 9 & \text { AMVSEFLKAAWFI D } \\ 11 & \text { AMVSEFLKQAAFI D } \\ 12 & \text { AMVSEFLKQAWAI D } \\ 13 & \text { AMVSEFLKQAWFAD } \\ 14 & \text { AMVSEFLKQAWFI A } \\ \text { REV DIFWAQKLFESVWA }\end{array}$

\begin{tabular}{ll}
\multicolumn{3}{c}{ AnnexinA2 } \\
WT & STVHEILSKLSLEG \\
WT & STVHEILSKLSLEG \\
WT & STVHEILSKLSLEG \\
1 & ATVHEILSKLSLEG \\
2 & SAVHEILSKLSLEG \\
3 & STAHEILSKLSLEG \\
4 & STVAEILSKLSLEG \\
5 & STVHAILSKLSLEG \\
6 & STVHEALSKLSLEG \\
7 & STVHEIASKLSLEG \\
8 & STVHEILAKLSLEG \\
9 & STVHEILSALSLEG \\
10 & STVHEILSKASLEG \\
11 & STVHEILSKLALEG \\
12 & STVHEILSKLSAEG \\
13 & STVHEILSKLSLAG \\
14 & STVHEILSKLSLEA \\
REV GELSLKSLIEHVTS
\end{tabular}

Figure 5.1 Amino acid sequences used for peptide array experiments.

Wild type (WT) sequences corresponding to the N-terminal region of annexin A1 and annexin A2 were synthesized, followed by alanine scanning mutagenesis of each peptide. The numbering in the left column corresponds to the position of the alanine substitution. Reverse sequences (REV) were used as negative controls. 
The dried membrane was incubated in anhydrous methanol and slowly hydrated with addition of water. The membranes were then rinsed with water and wash buffer containing $20 \mathrm{mM}$ Tris- $\mathrm{HCl}(\mathrm{pH} 7.6), 137 \mathrm{mM} \mathrm{NaCl} \mathrm{0.1 \% (v/v),} \mathrm{Tween} 20$ and $1 \mathrm{mM}$ $\mathrm{CaCl}_{2}$, followed by incubation in binding buffer (wash buffer with $5 \%(\mathrm{w} / \mathrm{v})$ sucrose) $\mathrm{CaCl}_{2}$, followed by $1 \mathrm{~h}$ incubation in binding buffer (wash buffer with $5 \%$ (w/v) sucrose) containing $10 \mathrm{nM}$ GST-S100A11. The binding buffer was removed and the membrane rinsed with wash buffer (twice for $5 \mathrm{~min}$ and once for $10 \mathrm{~min}$ ). The membrane was then blocked overnight at $4^{\circ} \mathrm{C}$ in blocking buffer (wash buffer with $1 \%$ BSA). Rabbit antiGST antibody (sigma) (1:2000) in blocking buffer was incubated with the membrane for 1h, washed and incubated with anti-rabbit IgG alkaline phosphatase linked antibody (1:10000). After washing, the antibodies were detected by ECF reagents following the manufacturers protocol (Amersham). Spots were visualized using a Bio-Rad Fluor-S MultiImager $(\lambda=530 \mathrm{~nm}$, aperture $=4$, exposure $=1.5 \mathrm{~s})$

\subsubsection{Isothermal Titration Calorimetry}

Thermodynamic parameters $\left(\mathrm{K}_{\mathrm{d}}, \Delta \mathrm{H}, \Delta \mathrm{S}, \Delta \mathrm{G}\right)$ were determined by measuring the heat of reaction during the titration of selected peptides into solutions of S100 protein or vice versa. All ITC experiments were performed on a MicroCal VP-ITC microcalorimeter (Microcal, Inc., Northampton MA). Proteins were dissolved in $20 \mathrm{mM}$ Pipes (pH 7.2), $50 \mathrm{mM} \mathrm{NaCl}, 0.2 \mathrm{mM}$ EDTA, $5 \mathrm{mM} \mathrm{CaCl}_{2}$ and $1 \mathrm{mM}$ TCEP, and then exhaustively dialyzed with three buffer exchanges at $4^{\circ} \mathrm{C}$. The binding peptides were dissolved in the final dialysis buffer. Protein and peptide concentrations were determined by using the alanine and leucine concentrations determined from triplicate amino acid 
analyses (Amino Acid Analysis Facility, Hospital of Sick Children, Toronto). The majority of ITC titrations were performed using $10 \mu \mathrm{L}$ additions of peptide solutions ranging in concentration between $0.10-0.80 \mathrm{mM}$. The aliquots were injected into the ITC cell containing the appropriate S100 binding partner. The concentration of the S100 protein in the cell varied between $0.01-0.150 \mathrm{mM}$ depending on the magnitude of the heat effects observed. In the case of annexin A1 the titrations were reversed (annexin A1 was in the ITC cell and S100A11 was used in the titration syringe), due to the low solubility of the peptide. Heats of dilution were measured in separate experiments in which the peptide was injected into buffer alone. This heat of dilution or the average of data points after saturation was subtracted before performing the curve fitting [8] and was found to be less than $1 \%$ of the heat effect for the S100 interaction. The stoichiometry $(\mathrm{N})$, association constant $(\mathrm{Ka})$, and change in enthalpy $(\Delta \mathrm{H})$ were determined from each data set. The change in entropy $(\Delta \mathrm{S})$ and Gibbs free energy $(\Delta \mathrm{G})$ were calculated according to equations 1 and 2.

$$
\begin{aligned}
& \Delta \mathrm{G}=-\mathrm{RT} \ln \mathrm{Ka} \\
& \Delta \mathrm{G}=\Delta \mathrm{H}-\mathrm{T} \Delta \mathrm{S}
\end{aligned}
$$

The titrations were performed at multiple temperatures in order to determine the change in heat capacity $(\Delta \mathrm{Cp})$ associated with the binding using equation 3.

$$
\Delta \mathrm{Cp}=\mathrm{d} \Delta \mathrm{H} / \mathrm{dT}
$$

The heat of reaction, (Q), was obtained by integrating the peak after each injection of ligand using Microcal Origin Software provided by the manufacturer. Data were fit to either one-site or two independent site models using Microcal Origin Software [9]. 


\subsubsection{Structure-Based Calculation of $\triangle C p$}

The changes in surface area upon S100-peptide partner complex formation were used to estimate the expected heat capacity change using the empirical relationship in equation 4 , where $\Delta \mathrm{ASA}_{\text {nonpolar, }} \Delta \mathrm{ASA}_{\text {polar and }} \Delta \mathrm{ASA}_{\mathrm{bb}}$ are the changes in accessible surface area between the peptide-bound and peptide-free forms of the complex for nonpolar, polar and backbone portions of the proteins respectively.

$$
\Delta \mathrm{Cp}=2.14 \Delta \mathrm{ASA}_{\text {nonpolar }}+0.88 \Delta \mathrm{ASA}_{\text {polar }}-1.81 \Delta \mathrm{ASA}_{\mathrm{bb}}
$$

Accessible surface areas were calculated using the three-dimensional structures for each complex using the program VADAR [10] and estimates for the surface area for the unfolded peptides $[11,12]$ as in equation 5 ,

$$
\Delta \mathrm{ASA}=\Delta \mathrm{ASA}_{\mathrm{S} 100-\text { pep }}-\Delta \mathrm{ASA}_{\mathrm{S} 100}-\Delta \mathrm{ASA}_{\text {pep }}
$$

where $\Delta \mathrm{ASA}_{\mathrm{S100-pep}}$ is the change in water accessible surface of the $\mathrm{S} 100$ protein-target peptide complex, $\Delta \mathrm{ASA}_{\mathrm{S} 100}$ is the change in water accessible surface area of the $\mathrm{S} 100$ protein and $\triangle \mathrm{ASA}_{\text {pep }}$ is the change in water accessible surface area for the unfolded peptide.

\subsection{Results and Discussion}

\subsubsection{Binding specificity of annexins $\mathrm{Al}$ and annexin $\mathrm{A2}$ to $\mathrm{Ca}^{2+}-S 100 \mathrm{Al} 1$}

Three-dimensional structures of the N-terminal peptide regions of annexin A1 and annexin A2 complexed to $\mathrm{Ca}^{2+}-\mathrm{S} 100 \mathrm{~A} 11$ (PDB Code 1QLS) [2] and S100A10 (1BT6) [1]respectively, reveal a great degree of similarity (rmsd $0.87 \AA$ ) between the two complexes (Figure 1.11). In both structures the annexin peptide bridges the two S100 
monomers, interacting with residues from helices III, IV and the linker region using the C-terminal portion and contacting the N-terminus of helix I' in the partner monomer via its N-terminal region. In addition, the annexin peptides form amphipathic helices upon complex formation and present three identical residues at positions Val3, Glu5 and Leu7 and conserved hydrophobic residues at positions Phe/Ile6 and Phe/Leu12 (Figure 5.2).

Despite similarities in structure and sequence, previous fluorescence experiments showed that the N-terminal annexin A1 peptide is unable to interact with S100A10 and that annexin $\mathrm{A} 2$ is a specific partner for $\mathrm{S} 100 \mathrm{~A} 10[2,13]$.

To determine if $\mathrm{Ca}^{2+}-\mathrm{S} 100 \mathrm{~A} 11$ is able to interact with the $\mathrm{N}$-terminal peptide of annexin A1 and A2 peptide array experiments were conducted. In these experiments peptides corresponding to $\mathrm{N}$-terminal sequences of annexin A1 or A2 were synthesized on a cellulose membrane and then probed for interaction with $\mathrm{Ca}^{2+}-\mathrm{S} 100 \mathrm{~A} 11$. Peptides were identical lengths and were acetylated at their N-termini, as the acetyl group is essential for the S100-annexin interaction. In addition, alanine scans of each amino acid were used to identify residues important in the interaction. It was expected that residues that are important in the interaction present modified affinity when substituted by alanine with respect to the wild-type interaction.

The annexin A1 and A2 peptide membranes are shown in Figure 5.3, with each square containing an individual peptide sequence. The $\mathrm{Ca}^{2+}-\mathrm{S} 100 \mathrm{~A} 11$ interaction with the peptide arrays was probed with GST-S100A11 in the presence of calcium and detected using anti-GST antibody and ECF fluorescence. Comparison of the spot intensities for the wild type sequences of annexin A1 and A2 showed moderate fluorescence of similar 


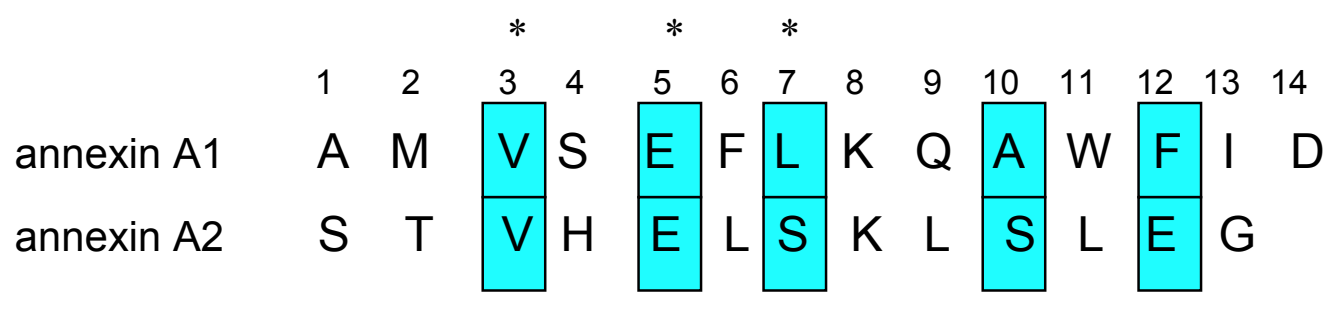

Figure 5.2 Sequence alignments of the $\mathrm{N}$-terminal sequences of annexin A1 and A2.

Identical residues are indicated with asterisks and hydrophobic residues at equivalent positions are highlighted in blue. Residues at positions 3, 6, 7 and 10 have been shown to be important for interactions with $\mathrm{Ca}^{2+}-\mathrm{S} 100 \mathrm{~A} 11$ (annexin A1) and S100A10 (annexin A2). 
intensity for both peptides in all three replicates. This result suggests that $\mathrm{Ca}^{2+}-\mathrm{S} 100 \mathrm{~A} 11$ binds to both annexin A1 and annexin A2 with a similar affinity. In Figure 5.3B the average intensities of the wild type annexin A1 and A2 spots (solid lines) and the average \pm one standard deviation (broken lines) are shown. When comparing binding efficiency, only intensities outside the standard deviation were considered significantly different form wild type.

The alanine substituted peptides arrays for both annexin A1 and A2 showed diverse degrees of binding as evident by differences in the spot intensities. In both peptide arrays, poor interaction was seen with the peptide sequence in the reverse order. This indicates that the direction of the sequence is essential for interaction with the binding surface of $\mathrm{Ca}^{2+}-\mathrm{S} 100 \mathrm{~A} 11$. Increased binding to $\mathrm{Ca}^{2+}-\mathrm{S} 100 \mathrm{~A} 11$ was observed for peptides M2A and S4A in annexin A1 compared to wild type. In the $\mathrm{Ca}^{2+}$ S100A11/annexin A1 crystal structure, Met2 and Ser4 make contacts with residues in helix I' (Ile14') and helix IV (Phe91) and the linker region (Ala45, Asn49) respectively. Alanine substitutions at positions 2 and 4 in the annexin A2 peptide also presented stronger intensity, suggesting similarities in the interactions. Similarly, alanine substitutions in residues 6 and 7 of both annexins presented decreased (F6A, I6A) or eliminated (L7A) binding to $\mathrm{Ca}^{2+}-\mathrm{S} 100 \mathrm{~A} 11$.

Examining the structure of $\mathrm{Ca}^{2+}-\mathrm{S} 100 \mathrm{~A} 11$ with annexin A1 it is evident that $\mathrm{Leu}^{7}$ makes several contacts with helix I' (Glu7' and Ile10') of one protomer and the linker (Leu43, Ala45 and Phe46) and helix IV (Leu83 and Cys87) of the other protomer. 
Interactions are seen also for Phe6 and side chains of residues in helix IV (Leu83 and

Ser90). Comparison with the S100A10/annexin A2 structure shows that Ile6 and Leu7
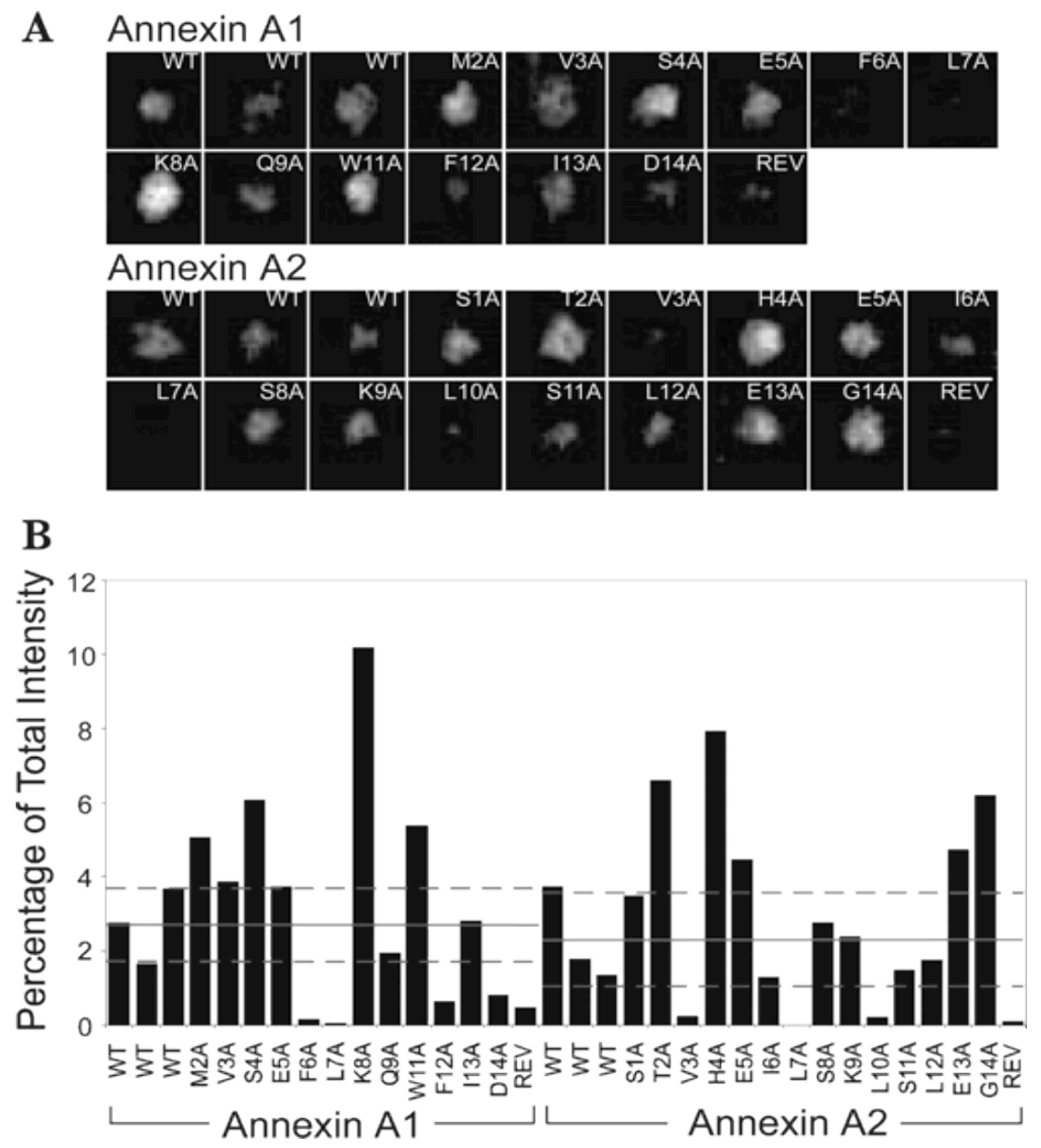

Figure 5.3 $\mathrm{Ca}^{2+}-\mathrm{S100A} \mathrm{A1}$ binding to annexin peptides shown by peptide arrays.

(A) Representative peptide array of 14-mer sequences of annexin A1 (AMVSEFLKQAWFID) and annexin A2 (STVHEILSKLSLEG) covalently bound to a cellulose membrane. Each square on the membrane contains an individual peptide sequence and it is labeled WT for the native sequence, the residue and the position of the alanine substitution, and REV for the native sequence in reverse orientation. The filter was incubated with GST-S100A11 in the presence of calcium and bound protein was detected by anti GST-antibody and chemifluorescence. (B) Histogram of the intensities measured using ImageJ software . Each bar represents the intensity of an individual spot 
as a fraction of the intensity of all the spots. The solid line corresponds to the average intensities, and broken lines are shown at one standard deviation from the average of the WT annexin A1 and A2 peptides $(2.70 \pm 1.02$ and $2.30 \pm 1.27)$. Experiments were performed in duplicate with similar results (data not shown).

make nearly identical contacts. The V3A and L10A peptides also presented decreased affinity for $\mathrm{Ca}^{2+}-\mathrm{S} 100 \mathrm{~A} 11$. These residues in addition to Leu7 make several contacts with the protein that are no longer possible with the shorter side chain present in alanine.

Previous work from Becker and coworkers [14] showed that replacement of the hydrophobic residues at positions 3,6, 7 and 10 in annexin A2 caused significant decreases in the affinity for S100A10. In a similar manner the peptide array studies provide evidence of the importance of these hydrophobic residues for the interaction of annexins $\mathrm{A} 1$ and $\mathrm{A} 2$ with $\mathrm{Ca}^{2+}-\mathrm{S} 100 \mathrm{~A} 11$. This indicates that the $\mathrm{N}$-termini of annexinsA1 and A2 use a similar motif to bind both S100A10 and $\mathrm{Ca}^{2+}-\mathrm{S} 100 \mathrm{~A} 11$.

From the peptide array experiments it is clear that both N-termini of annexins A1 and A2 interact with S100A11 in a calcium dependent manner. This places S100A11 in a group of S100 proteins that includes S100A6 that have been shown to interact with multiple members of the annexin family. It is interesting that previous fluorescence experiments reported only non-specific interactions for $\mathrm{Ca}^{2+}-\mathrm{S} 100 \mathrm{~A} 11$ and an annexin A2 peptide labeled with Prodan at Cys8. It is possible that the fluorescent Prodan tag contributed to the lack of interaction in these previous studies.

S100-annexin complexes are proposed to facilitate membrane fusion events. This process requires not only the S100-annexin complex formation but also involves a calcium-sensitive binding of annexin to phospholipid on the membrane surface. The ability of $\mathrm{Ca}^{2+}-\mathrm{S} 100 \mathrm{~A} 11$ to associate with both annexins A1 and A2 could imply a certain 
level of diversity to the membrane fusion process, as one S100 would be able to recruit different annexin molecules.

\subsubsection{Annexin A1 and A2 interactions with $\mathrm{Ca}^{2+}-S 100 \mathrm{Al} 1$ are thermodynamically distinct}

$\mathrm{Ca}^{2+}-\mathrm{S} 100 \mathrm{~A} 11$ has been shown two interact with relatively high affinity $(\mu \mathrm{M}$ range) with the N-terminal sequences of annexin A1 and annexin A2 ([15] and preceding sections of this chapter) using a similar binding surface. Because of the similarity of the $\mathrm{Ca}^{2+}-\mathrm{S} 100 \mathrm{~A} 11$ interaction with annexins $\mathrm{A} 1$ and $\mathrm{A} 2$, it is possible that the relative contributions to binding (burial of hydrophobic residues, solvation effects and small conformational changes) could provide insight for the modes of interaction of each annexin peptide. Thus, a detailed calorimetric investigation of the binding of annexins A1 and $\mathrm{A} 2$ to $\mathrm{Ca}^{2+}-\mathrm{S} 100 \mathrm{~A} 11$ was conducted.

Isothermal titration calorimetry (ITC) examines the binding equilibrium by measuring the heat absorbed or released upon ligand binding. This allows direct measurement the enthalpy of binding, the association constant and the stoichiometry of the binding interaction. Figure 5.3 shows representative ITC profiles for the titration of annexin A2 into $\mathrm{Ca}^{2+}-\mathrm{S} 100 \mathrm{~A} 11$ and $\mathrm{Ca}^{2+}-\mathrm{S} 100 \mathrm{~A} 11$ into annexin A1. Table 5.1 summarizes the thermodynamic parameters of the $\mathrm{Ca}^{2+}-\mathrm{S} 100 \mathrm{~A} 11$ interactions with the annexin peptides. S100A10-annexin A2 was also included for comparison.

ITC experiments confirmed the interaction between $\mathrm{Ca}^{2+}-\mathrm{S} 100 \mathrm{~A} 11$ and annexin peptides A1 and A2 and also showed that the additional C-terminal residues (NEERR) in the annexin A1 peptide do not impair binding (Figure 5.4A). The interaction of $\mathrm{Ca}^{2+}$ S100A11 with the N-terminal peptides from annexins A1 and A2 exhibited similar 
affinities $\left(\mathrm{K}_{\mathrm{d}}=6.6 \mu \mathrm{M}\right.$ and $4.12 \mu \mathrm{M}$ respectively) and little temperature dependence (Table 5.1). Therefore similar binding free energies changes were obtained over the temperature range studied. In both cases the interaction of $\mathrm{Ca}^{2+}-\mathrm{S} 100 \mathrm{~A} 11$ with annexin peptides was best fit to a single site-binding model with one molecule of peptide binding per monomer of $\mathrm{Ca}^{2+}-\mathrm{S} 100 \mathrm{~A} 11$. This can be seen in the calorimetric traces in Figure 5.4 where the heat responses gradually decrease until saturation is reached, after this point the effects are due solely to dilution of the peptide. In addition, the fitted stoichiometries for the association were all near 1.0 indicating the correct binding model was used.

A recent calorimetric study [16] which screened some S100 proteins for annexin A1 and A2 peptide binding reported a 1:1 stoichiometry as well for the interactions, but found a better fit with a sequential two-site binding model (2:2, peptide:S100 protomer). One important difference from the current work is that the experiments reported by Streicher et al. were conducted in $20 \mathrm{mM}$ Tris at $\mathrm{pH}$ 7.5. This buffer has a high enthalpy of ionization $\left(\Delta \mathrm{H}_{\mathrm{ion}}=-47.45 \mathrm{~kJ} / \mathrm{mol}\right)$, which leads to greater effects linked to protonation and can complicate data fitting. The experimentally measured $\Delta \mathrm{H}$ consists of enthalpy of binding $(\Delta \mathrm{Hb})$ and the enthalpy linked to protonation effects $(\Delta \mathrm{Hp})$, resulting from buffer containing $10 \mathrm{mM}$ Tris $\mathrm{pH} 7.2$ the heat signal was cancelled for the reaction due to the endothermic nature of the heat of dilution for annexin A2. (Figure 5.5). This could explain why ITC measurements for $\mathrm{Ca}^{2+}-\mathrm{S} 100 \mathrm{~A} 11$ and annexin A2 by Streicher et. al. [16] were performed below $25^{\circ} \mathrm{C}$. The $\Delta \mathrm{H}$ values reported by Streicher and coworkers correspond to the enthalpy of binding with a high contribution from the protonation effects. In the studies described here the buffer (20 mM Pipes) was chosen, which has a 

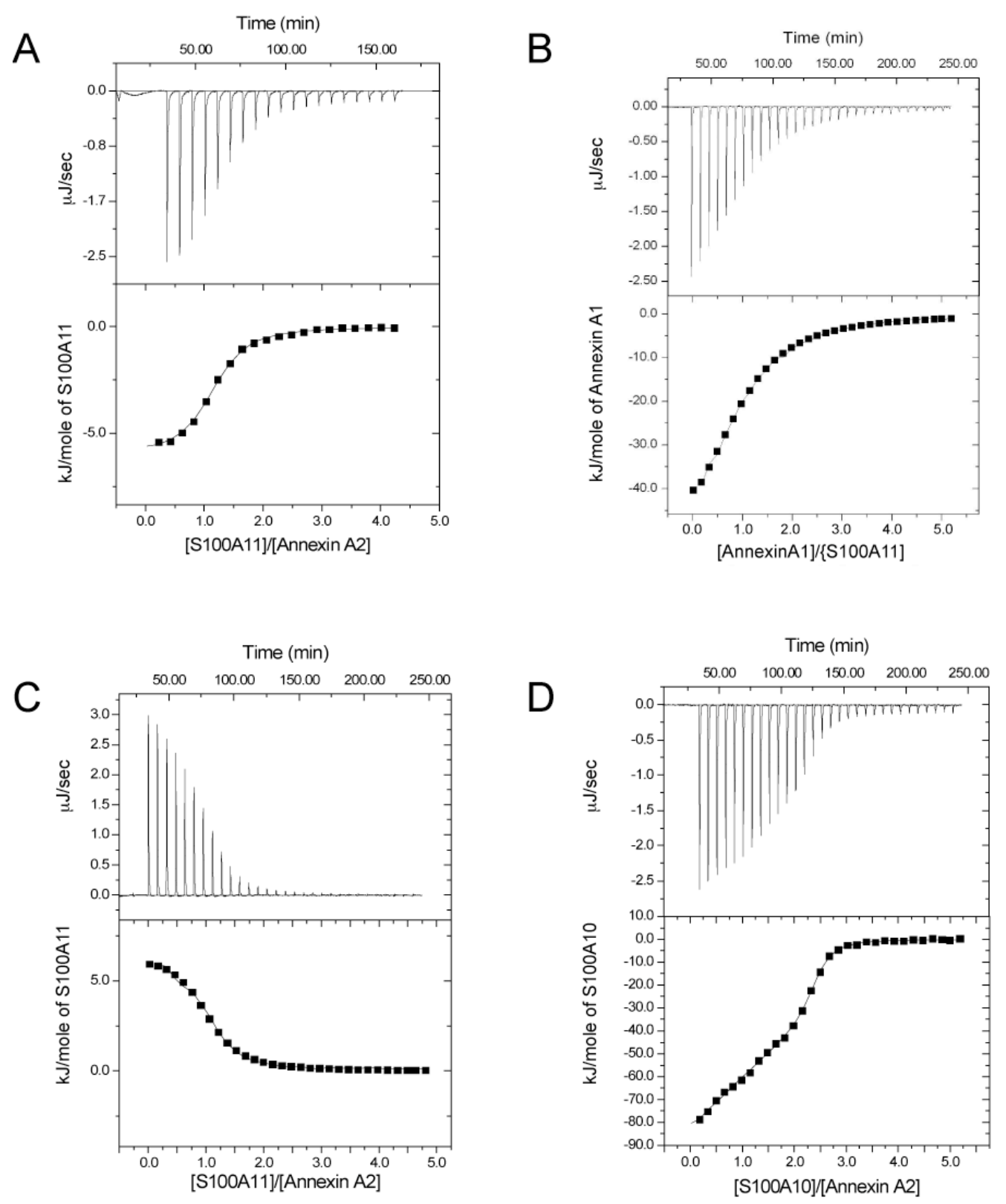

Figure 5.4 ITC experiments for annexin peptide binding to $\mathrm{Ca}^{2+}-\mathrm{S} 100 \mathrm{~A} 11$.

(A) Annexin A1 titrated with $\mathrm{Ca}^{2+}-\mathrm{S} 100 \mathrm{~A} 11$ at $25^{\circ} \mathrm{C}$ (B) $\mathrm{Ca}^{2+}-\mathrm{S} 100 \mathrm{~A} 11$ titrated with annexin $\mathrm{A} 2$ at $25^{\circ} \mathrm{C}(\mathrm{C})$ annexin $\mathrm{A} 1$ titrated with $\mathrm{Ca}^{2+}-\mathrm{S} 100 \mathrm{~A} 11$ at $17^{\circ} \mathrm{C}$ (D) S100A10 titrated with annexin $\mathrm{A} 2$ at $25^{\circ} \mathrm{C}$. Experiments were performed in $20 \mathrm{mM}$ Pipes (pH 7.2), $50 \mathrm{mM} \mathrm{NaCl}, 0.2 \mathrm{mM}$ EDTA, $5 \mathrm{mM} \mathrm{CaCl}_{2}$ and $1 \mathrm{mM}$ TCEP. The top panels display the 
baseline corrected calorimetric data while the bottom panels display the derived binding isotherm for each experiment.

Table 5.1Thermodynamic parameters for Titrations of $\mathrm{Ca}^{2+}-\mathrm{S} 100 \mathrm{~A} 11$ with annexin A1 and $A 2$ peptides

\begin{tabular}{|c|c|c|c|c|c|c|c|}
\hline Protein & Target & $\begin{array}{c}\text { Temp } \\
\left({ }^{\circ} \mathrm{C}\right)\end{array}$ & $\mathbf{N}$ & $\begin{array}{c}\mathbf{K d} \\
(\mu \mathrm{M})\end{array}$ & $\begin{array}{c}\Delta \mathbf{H} \\
(\mathrm{kJ} / \mathrm{mol})\end{array}$ & $\begin{array}{c}T \Delta S \\
(\mathrm{~kJ} / \mathrm{mol})\end{array}$ & $\underset{(\mathbf{k J} / \mathbf{m o l})}{\Delta G}$ \\
\hline \multirow[t]{4}{*}{ S100A11 } & annexin A1 & 17 & 1.04 & 4.26 & -54.81 & -25.24 & -29.82 \\
\hline & & 25 & 1.05 & 6.94 & -65.27 & -36.89 & -29.92 \\
\hline & & 30 & 1.01 & 12.39 & -69.04 & -39.65 & -27.99 \\
\hline & & 35 & 1.04 & 2.82 & -80.75 & -48.20 & -32.73 \\
\hline \multirow[t]{4}{*}{ S100A11 } & annexin A2 & 17 & 1.02 & 7.25 & 6.50 & 35.07 & -28.53 \\
\hline & & 25 & 1.12 & 4.63 & -6.03 & 24.44 & -30.43 \\
\hline & & 30 & 0.99 & 1.45 & -23.70 & 9.77 & -33.86 \\
\hline & & 35 & 1.03 & 3.14 & -30.72 & 1.74 & -32.44 \\
\hline \multirow[t]{2}{*}{ S100A10 } & annexin A2 & 25 & 0.75 & 0.06 & -232.63 & -190.77 & -41.38 \\
\hline & & & 1.56 & 0.98 & 27.87 & 68.20 & -34.28 \\
\hline
\end{tabular}



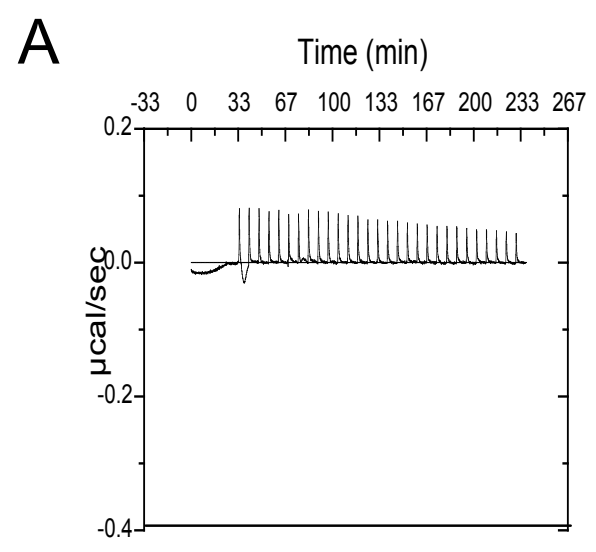

Time (min)

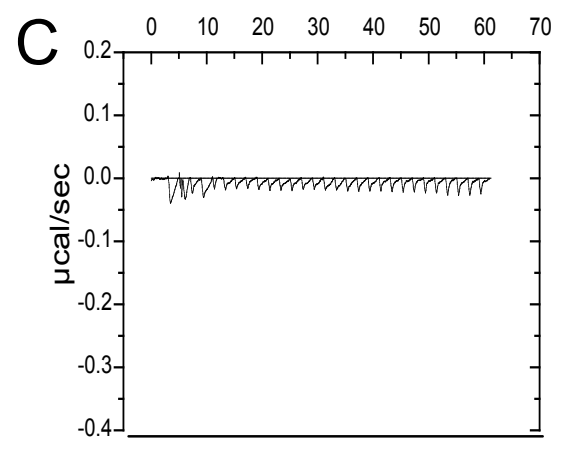

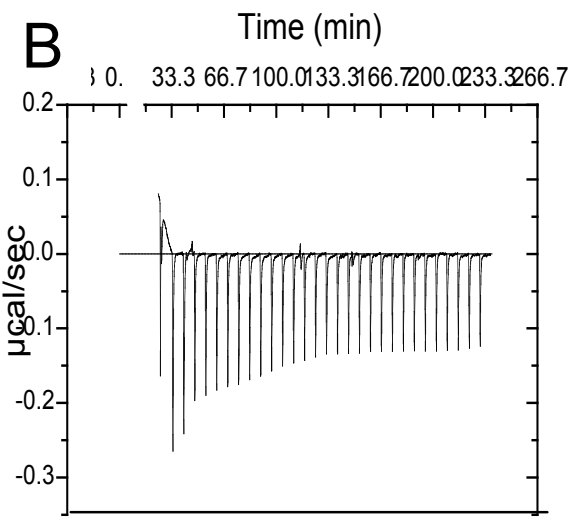

Figure 5.5 ITC experiments for annexin $\mathrm{A} 1$ and $\mathrm{A} 2$ binding to $\mathrm{Ca}^{2+}-\mathrm{S} 100 \mathrm{~A} 11$ in buffer containing 20mM Tris (pH 7.5)

Calorimetric traces of (A) annexin A2 into buffer alone (B) Buffer titrated into $\mathrm{Ca}^{2+}$ S100A11 and (C) $\mathrm{Ca}^{2+}-\mathrm{S} 100 \mathrm{~A} 11$ titrated with annexin A2 peptide. 
much smaller enthalpy of ionization $\left(\Delta \mathrm{H}_{\mathrm{ion}}=12 \mathrm{~kJ} / \mathrm{mol}\right)$ and minimizes the protonation effects.

The complex formation of $\mathrm{Ca}^{2+}-\mathrm{S} 100 \mathrm{~A} 11$ with annexin $\mathrm{A} 1$ under all conditions tested was exothermic. In contrast, binding of annexin $\mathrm{A} 2$ with $\mathrm{Ca}^{2+}-\mathrm{S} 100 \mathrm{~A} 11$ at $17^{\circ} \mathrm{C}$ shows a calorimetric trace representative of an endothermic reaction, suggesting a different contribution of the enthalpy in the complex formation of S100A11 and the annexins in the presence of calcium. In fact, as seen in Table 5.1 the interaction of annexin $\mathrm{A} 1$ and annexin $\mathrm{A} 2$ with $\mathrm{Ca}^{2+}-\mathrm{S} 100 \mathrm{~A} 11$ are thermodynamically distinct, with change in entropy (Figure 5.6). The X-ray structure of $\mathrm{Ca}^{2+}-\mathrm{S} 100 \mathrm{~A} 11$ in complex with annexin A1 shows that upon binding the annexin A1 acquires an $\alpha$-helical fold. Peptides are substantially more disordered in the unbound state, with annexin A1 existing as random coil in solution and taking on an ordered helical conformation upon binding. This coil to helix transition upon binding is accompanied by a large entropic cost, compensated by a large negative enthalpic contribution.

The three dimensional structure of the complex $\mathrm{Ca}^{2+}-\mathrm{S} 100 \mathrm{~A} 11$-annexin A2 has not yet been reported, but given the similarity between the target sequences (Figure 5.2) and the structural similarities between S100A10 and $\mathrm{Ca}^{2+}-\mathrm{S} 100 \mathrm{~A} 11$, a similar coil-helix transition for annexin A2 upon binding to $\mathrm{Ca}^{2+}-\mathrm{S} 100 \mathrm{~A} 11$ could be expected. However, the thermodynamics for this complex suggest that the transition does not take place when annexin A2 binds to S100A11 in the presence of calcium. This complex formation is entropically favourable with a small negative contribution from the enthalpy to the free 
energy of binding suggesting either the peptide binds in a different conformation or a larger rearrangement of some side chains occurs in S100A11.

For comparison S100A10 was titrated with annexin A2 (Figure 5.4D). The main observation is that complex formation proceeded with a large release of heat, suggesting an important enthalpic contribution to free energy of binding. In contrast, to the interaction of S100A11 with the annexin peptides, a two-site model best described the complex of S100A10 with annexin A2. Despite the homodimeric nature of the S100 proteins previous work has suggested a possible allosteric effect upon ligand binding such as that evident in the ITC data for the complex of S100A10-annexin A2. Thermodynamically S100A10-annexin A2 is more similar to the complex formation between $\mathrm{Ca}^{2+}-\mathrm{S} 100 \mathrm{~A} 11$ and annexin A1, characterized by a coil to helix transition for the annexin peptide and a large entropic cost compensated by a large negative enthalpic contribution.

Overall the thermodynamic binding for the annexin peptides to both $\mathrm{Ca}^{2+}$ S100A11 and S100A10 displayed string enthalpy-entropy compensation. Therefore, the changes in $\Delta \mathrm{H}$ and $\mathrm{T} \Delta \mathrm{S}$ offset to give similar binding free energy changes over the entire temperature range studied ( $\Delta \mathrm{G} \sim 30 \mathrm{~kJ} / \mathrm{mol}$ ). The slope of the enthalpy change with temperature was used to calculate the change in heat capacity $(\Delta \mathrm{Cp})$ for binding of each peptide to $\mathrm{Ca}^{2+}-\mathrm{S} 100 \mathrm{~A} 11$, showing the strong dependence of enthalpy with temperature (Table 5.1). Both complexes exhibited a negative change in heat capacity $(\Delta \mathrm{Cp}=-2.2 \pm$ $0.16 \mathrm{~kJ} / \mathrm{mol}{ }^{\circ} \mathrm{K}$ for $\mathrm{Ca}^{2+}-\mathrm{S} 100 \mathrm{~A} 11$-annexin $\mathrm{A} 2$ and $-1.33 \mathrm{~kJ} / \mathrm{mol}{ }^{\circ} \mathrm{K}$ for $\mathrm{Ca}^{2+}-\mathrm{S} 100 \mathrm{~A} 11-$ annexin A1), which is an indicator of the burial of hydrophobic residues upon complex 


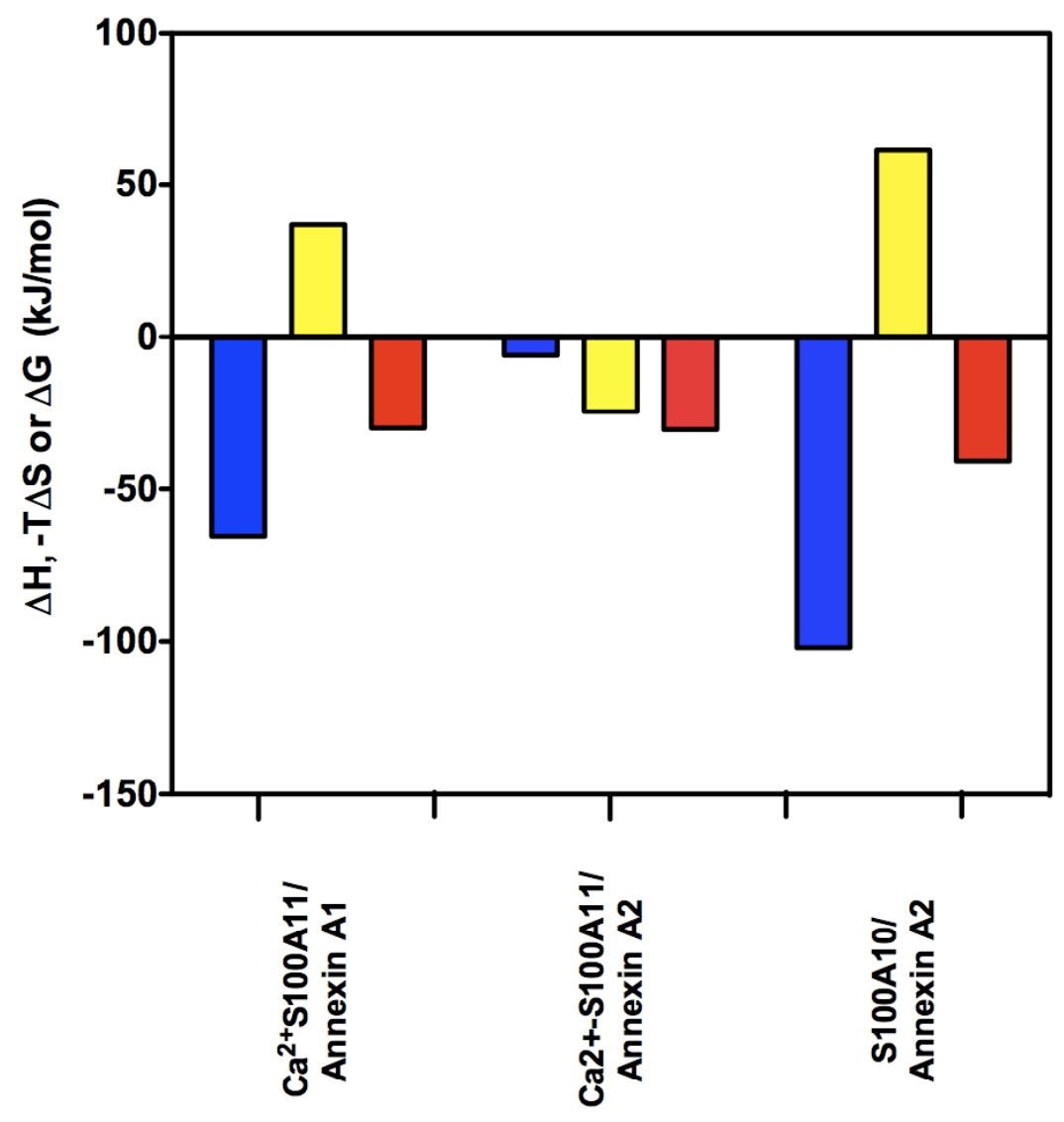

Figure 5.6 Comparison of thermodynamic parameters for S100-target interactions

Comparison of the enthalpic $(\Delta \mathrm{H}$, blue bars) and entropic (-T $\Delta \mathrm{S}$, yellow bars) to the Gibbs free energy (red bars) of peptide/S100 proteins at $25{ }^{\circ} \mathrm{C}$. 
formation inferred form three-dimensional structure data $(\Delta \mathrm{Cp}=-2.03 \mathrm{~kJ} / \mathrm{mol} \mathrm{K}$ for $\mathrm{Ca}^{2+}-\mathrm{S} 100 \mathrm{~A} 11$-annexin $\mathrm{A} 2$ and $-2.6 \mathrm{~kJ} / \mathrm{mol} \mathrm{K}$ for $\mathrm{Ca}^{2+}-\mathrm{S} 100 \mathrm{~A} 11$-annexin A1). The $\Delta \mathrm{Cp}$ experimental for $\mathrm{Ca}^{2+}-\mathrm{S} 100 \mathrm{~A} 11 /$ annexin $\mathrm{A} 2$ is slightly more negative than for the complex in the presence with annexin A1, which could suggest that the hydrophobic pocket for binding to annexin A2 is slightly larger.

\subsection{Conclusions}

A new interaction between $\mathrm{Ca}^{2+}-\mathrm{S} 100 \mathrm{~A} 11$ and annexin $\mathrm{A} 2$ was shown by peptide array assays and isothermal titration calorimetry. In the presence of calcium both annexins $\mathrm{A} 1$ and $\mathrm{A} 2$ both form tight complexes with $\mathrm{S} 100 \mathrm{~A} 11(\mathrm{Kd} \sim 5 \mu \mathrm{M})$. ITC data shows that upon binding of $\mathrm{Ca}^{2+}-\mathrm{S} 100 \mathrm{~A} 11$, annexin $\mathrm{A} 1$ adopts a helical conformation, but annexin A2 appears to retain a different conformation. The results show an important enthalpy/entropy balance for the stabilization of S100-annexin protein complexes. 


\subsection{References}

1 Rety, S., Sopkova, J., Renouard, M., Osterloh, D., Gerke, V., Tabaries, S., RussoMarie, R. and Lewit-Bentley, A. (1999) The crystal structure of a complex of p11 with the annexin II N-terminal peptide. Nature Struct. Biol. 6, 89-95

2 Rety, S., Osterloh, D., Arie, J. P., Tabaries, S., Seeman, J., Russo-Marie, F., Gerke, V. and Lewit-Bentley, A. (2000) Structural basis of the $\mathrm{Ca}(2+)$-dependent association between S100C (S100A11) and its target, the N-terminal part of annexin I. Structure Fold Des. 8, 175-184

3 Rustandi, R. R., Baldisseri, D. M. and Weber, D. J. (2000) Structure of the negative regulatory domain of p53 bound to $\mathrm{S} 100 \mathrm{~B}(\mathrm{bb})$. Nat. Struct. Biol. 7, 570-574

4 McClintock, K. A., Van Eldik, L. J. and Shaw, G. S. (2002) The C-terminus and linker region of S100B exert dual control on protein-protein interactions with TRTK12. Biochemistry. 41, 5421-5428

5 Bhattacharya, S., Large, E., Heizmann, C. W., Hemmings, B. and Chazin, W. J. (2003) Structure of the Ca2+/S100B/NDR kinase peptide complex: insights into S100 target specificity and activation of the kinase. Biochemistry. 42, 14416-14426

6 Wright, N. T., Prosser, B. L., Varney, K. M., Zimmer, D. B., Schneider, M. F. and Weber, D. J. (2008) S100A1 and calmodulin compete for the same binding site on ryanodine receptor. J Biol Chem. 283, 26676-26683

7 Lee, Y. T., Dimitrova, Y. N., Schneider, G., Ridenour, W. B., Bhattacharya, S., Soss, S. E., Caprioli, R. M., Filipek, A. and Chazin, W. J. (2008) Structure of the S100A6 complex with a fragment from the C-terminal domain of Siah-1 interacting protein: a novel mode for S100 protein target recognition. Biochemistry. 47, 10921-10932

8 Pierce, M. M., Raman, C. S. and Nall, B. T. (1999) Isothermal titration calorimetry of protein-protein interactions. Methods. 19, 213-221

9 Wiseman, T., Williston, S., Brandts, J. F. and Lin, L. N. (1989) Rapid measurement of binding constants and heats of binding using a new titration calorimeter. Anal Biochem. 179, 131-137

10 Willard, L., Ranjan, A., Zhang, H., Monzavi, H., Boyko, R. F., Sykes, B. D. and Wishart, D. S. (2003) VADAR: a web server for quantitative evaluation of protein structure quality. Nucleic Acids Res. 31, 3316-3319

11 Creamer, T. P., Srinivasan, R. and Rose, G. D. (1995) Modeling unfolded states of peptides and proteins. Biochemistry. 34, 16245-16250

12 Creamer, T. P., Srinivasan, R. and Rose, G. D. (1997) Modeling unfolded states of proteins and peptides. II. Backbone solvent accessibility. Biochemistry. 36, 28322835

13 Naka, M., Qing, Z. X., Sasaki, T., Kise, H., Tawara, I., Hamaguchi, S. and Tanaka, T. (1994) Purification and characterization of a novel calcium-binding protein, S100C, from porcine heart. Biochim Biophys Acta. 1223, 348-353

14 Becker, T., Weber, K. and Johnsson, N. (1990) Protein-protein recognition via short amphiphilic helices; a mutational analysis of the binding site of annexin II for p11. Embo J. 9, 4207-4213 
15 Rintala-Dempsey, A. C., Santamaria-Kisiel, L., Liao, Y., Lajoie, G. and Shaw, G. S. (2006) Insights into S100 target specificity examined by a new interaction between S100A11 and annexin A2. Biochemistry. 45, 14695-14705

16 Streicher, W. W., Lopez, M. M. and Makhatadze, G. I. (2009) Annexin I and annexin II N-terminal peptides binding to S100 protein family members: specificity and thermodynamic characterization. Biochemistry. 48, 2788-2798 


\section{Chapter 6 \\ SUMMARY AND PERSPECTIVES}

\subsection{Introduction}

The S100 proteins comprise a group of 25 members within the EF-hand calciumbinding protein family. Most of these dimeric proteins have been shown to act as calcium-signaling molecules, in a manner similar to that of calmodulin or troponin- $\mathrm{C}$, whereby the protein binds calcium and undergoes a conformational change [1-3]. Each S100 protein can interact with a diverse array of target proteins giving rise to a wide range of biological responses [4]. S100 proteins are proposed to be involved in the calcium dependent regulation of a variety of intracellular processes such as protein phosphorylation, cell growth and motility, cell cycle regulation, transcription and differentiation [5], however, their precise biological roles remain uncertain. The interest in S100 proteins has increased rapidly in the past few years due to their association with several human diseases including cancer, Alzheimer's, neurodegenerative disorders and cardiomyopathies, usually due to modified levels of expression of the S100 members [6] [7]. Also, a potential use in diagnosis and as drug targets is being explored.

S100A11 is one member of the S100 protein family proposed to facilitate membrane vesicle fusion events, such as those found in endocytosis and membrane repair, through calcium-dependent recruitment of annexins A1 and A2 [8-11]. The threedimensional structures of apo-S100A11 and $\mathrm{Ca}^{2+}-\mathrm{S} 100 \mathrm{~A} 11$ are representative of most other S100 proteins showing a calcium-induced conformational change essential for target recognition $[12,13]$. In contrast to the calcium-dependent conformational change 
for S100A11, S100A10 is a unique member of the S100 family that is unable to bind calcium due to substitutions and deletions of calcium-coordinating residues in both EFhands. Despite this deficiency, S100A10 in its calcium-free state has a similar "open" conformation to the structures of other S100 proteins in their calcium-bound forms, particularly $\mathrm{Ca}^{2+}-\mathrm{S} 100 \mathrm{~A} 11$ [12-14]. Thus, S100A10 interactions with target molecules are calcium insensitive and can be monitored in vivo.

Understanding the biological functions of S100 proteins depends critically on the identification of their target partners under physiological conditions, as seen for S100A10. Understanding the molecular basis of the active form in S100A10 would allow the design of S100 proteins and would serve as useful probes for the identification of in vivo targets and to elucidate their functional roles in $\mathrm{Ca}^{2+}$-signaling.

\subsection{Previous work}

Attempting to understand the connection between the sequence specific residueresidue interactions and the conformation of EF-hand proteins has been a goal of researchers for many years. The majority of efforts have been placed on identifying the residues that contribute most strongly to calcium binding within a calcium-binding loop. Using sequence conservation information and the precise geometry for calciumcoordination it has been possible to engineer or modulate the affinity of calcium-binding sites in a variety of proteins including lysozyme [15], CD2 [16] and kinase-inducible domains [17]. Further, site-directed mutations have elegantly shown how calcium-binding sites such as those in calbindin $\mathrm{D}_{9 \mathrm{k}}$ or troponin $\mathrm{C}$ act to modulate calcium-affinity [18-20] and its impact on the conformational change [21-24]. 
However, fewer examples exist where the overall conformation of an EF-hand calcium-binding protein has been altered. Bunick and co-workers showed that substitution of roughly $20 \%$ of the protein sequence in calbindin $\mathrm{D}_{9 \mathrm{k}}$ produced a modified protein (calbindomodulin) that was folded and adopted a more open calmodulin-like structure having a larger exposed hydrophobic surface upon binding of calcium, not present in the parent protein [25]. More recently, substitutions in S100P have allowed this protein to interact with one of its targets (ezrin) in the absence of calcium suggesting this modified protein has stabilized the open conformation [26]. However, this work examined only one set of substitutions making the contributions of different residues and regions towards the open conformation and target binding difficult to assess.

\subsection{Comparative studies of S100A10 and S100A11}

To help identify the sequences and regions responsible for the open conformation of S100A10 a combination of $\mathrm{C} \alpha$ distances, residue-residue contacts, hydrophobic surface area exposure and three-dimensional structure examination was used. The main dissimilarities in sequence were observed in the calcium binding loops where S100A10 carries deletions and substitutions of calcium coordinating residues. Moreover, the packing of interhelical faces involving helix III (II/III and III/IV) are significantly different in apo-S100A11 and S100A10. In apo-S100A11 the C-terminus of helix III comes into close proximity with the N-terminus of helix II, disrupting the interactions at the top of the helices. On the other face, the near perpendicular orientation of helix III with respect to helix IV in S100A10 disrupts a large number of hydrophobic residues along the helical interface that held together theses helices in the apo-form and are lost 
upon binding of calcium. It is possible that some of the residues forming the interhelical faces (II/III and III/ N-terminus of helix IV) contribute to the open conformation seen in S100A10. Of particular interest could be the presence of a unique salt bridge in S100A10, between D52 in helix III and R31 (L in S100A11) in helix II. Moreover an extensive network of hydrogen bonds was identified in both calcium-binding loops that seem to stabilize the loop in the open conformation of S100A10. For example, in the N-terminal hand EF-hand of S100A10 the presence of K22 allows the formation of hydrogen bonds with D29 and Y24 that are not formed in apo-S100A11. In site II, new hydrogen bonds are formed between the side chains of D59 and D63 and the main chains of S70 and G67, amongst others. In S100A10 unique hydrogen bonds are formed between the EF-hand loops and helix III (R31-K27; D52,K27; D57-Q60) and between helix III and II (D52R31) and the linker (K53-D47). These interactions could be critical in the stabilization of the open conformation in S100A10.

\subsection{Engineering of chimaeric S100A11/S100A10 proteins}

The sequence and structure of S100A10, $\mathrm{Ca}^{2+}$-S100A11 and apo-S100A11 were used to identify regions that could allow apo-S100A11 to assume a more open conformation, as for S100A10. Based on the regions where larger differences were found a set of seven chimaeric proteins that incorporated distinct regions of the S100A10 sequence into the S100A11 protein were engineered.

Changes in calcium-binding loop I (three amino acid deletion) and loop II (N66C, E75S) were made, as in S100A10 these substitutions impair calcium binding. These were not expected to impact the packing of helix III/IV, where multiple contacts between the 
N-terminus of helix III (V55, L56) and the C-terminus of helix IV (L83, A86, C87) were observed in apo-S100A11 but absent in the open forms of S100A10 and $\mathrm{Ca}^{2+}-\mathrm{S} 100 \mathrm{~A} 11$. These long-range contacts involving helix III suggested that interconnected substitutions would be required to reach an active conformation. A set of seven chimaeric proteins that incorporated distinct regions of the S100A10 sequence into the S100A11 protein were engineered. In general the chimaeric proteins designed could be divided into two groups those that had substitutions made in helices II, III and IV and those that had substitutions in calcium-binding sites I or II. All proteins were expressed and purified after the development of new methods since the chimaeric S100A11 proteins presented altered properties compared to the parental protein.

\subsection{Characterization of chimaeric proteins}

A goal of the chimaeric protein design was to examine how different regions of S100A10 (calcium-binding loops, helices II, III and IV) might modify the tertiary (ie. relative conformation of helices III and IV) structure when substituted into S100A11. As an initial step, the structural integrity of the chimaeric proteins was evaluated since several of these proteins had large sections of their sequences replaced that could potentially disrupt their folding. Dynamic light scattering and sedimentation equilibrium experiments confirmed that all chimaeric proteins remained as dimers similar to S100A11 and S100A10. Further, circular dichroism (CD) spectropolarimetry probed the proper folding and secondary structure of the seven chimaeric proteins. All proteins displayed characteristic $\alpha$-helical spectra with two minima near $208 \mathrm{~nm}$ and $222 \mathrm{~nm}$, indicating that substitutions did not disrupt the overall helical structure. Moreover, NMR 
spectroscopy and hydrophobicity assays for the chimaeric proteins in the presence of calcium showed that the proteins with helical regions $\left(\mathrm{S} 100 \mathrm{~A} 11^{\mathrm{H} 2, \mathrm{H} 3}, \mathrm{~S} 100 \mathrm{~A} 11^{\mathrm{H} 2 \mathrm{~L}, \mathrm{H} 3}\right.$, S100A $11^{\mathrm{H} 3, \mathrm{H} 4}$ ) substituted are able to adopt a calcium-induced open conformation, implying that large sequence changes in the helices of S100A11 do not prevent it from binding calcium nor undergoing a large conformational change. In contrast inactivation of the calcium binding loops in S100A11 (S100A11 ${ }^{\mathrm{LI}}, \mathrm{S} 100 \mathrm{~A} 11^{\mathrm{N} 66 \mathrm{C}, \mathrm{E} 75 \mathrm{~S}}, \mathrm{~S} 100 \mathrm{~A} 11^{\mathrm{LII}}$, S100A $11^{\text {LI,LII }) ~ l a r g e l y ~ r e d u c e s ~ t h e ~ c a l c i u m ~ a f f i n i t y ~ a n d ~ c o n s e q u e n t l y ~ i m p a i r s ~ t h e ~ o p e n i n g ~}$ of the structure.

\subsection{Evidence for a calcium-independent hydrophobic surface in loop II chimaeric proteins}

Since exposure of hydrophobic residues is characteristic of the open conformations in $\mathrm{S} 100 \mathrm{~A} 10$ and $\mathrm{Ca}^{2+}-\mathrm{S} 100 \mathrm{~A} 11$, the chimaeric S100A11 proteins were probed for exposed hydrophobicity using the hydrophobic probe ANS and a phenyl Sepharose matrix. Increased ANS fluorescence and phenyl Sepharose binding for the apo-S100A11 proteins having substitutions in their calcium binding sites was observed, suggesting a greater ability to occupy an open conformation in these proteins. Further, calcium independent binding to annexin A2 (GST-annexin A2) was used to elucidate if the increase in hydrophobic surface area lead to the exposure of the annexin A2 binding site. Apo-S100A $11^{\text {LII }}$ was the only chimaeric protein tested that exhibited increased hydrophobicity compared to apo-S100A11, and the ability to bind to annexin A2. This suggests that substitutions in site II may increase the population of the open state in S100A $11^{\text {LII }}$ similar to either S100A10 or $\mathrm{Ca}^{2+}-\mathrm{S} 100 \mathrm{~A} 11$. 


\subsection{Structural effects of substitutions in S100A11}

NMR spectroscopy experiments using chemical shift mapping and residual dipolar coupling revealed important structural differences of S100A11 ${ }^{\mathrm{LII}}$ in the absence of calcium respect to apo-S100A11. Residual dipolar coupling profiles for the helices of apo-S100A1 ${ }^{\mathrm{LII}}$ and apo-S100A11 identified the major structural differences in the helices II and III. The RDCs of S100A11 ${ }^{\mathrm{LII}}$ the chimaeric protein did not fit either structures of apo-S100A11 or $\mathrm{Ca}^{2+}-\mathrm{S} 100 \mathrm{~A} 11$. This could be suggestive of a structure intermediate or of an averaging of structures between the closed and open forms.

\subsection{Importance of calcium-binding site II to the open conformation of S100A10}

Of the seven S100A11 hybrid proteins designed, only apo-S100A11 ${ }^{\mathrm{N} 66 \mathrm{C}, \mathrm{E} 75 \mathrm{~S}}$ and apo-S100A11 ${ }^{\text {LII }}$ consistently showed significant increases in hydrophobic surface exposure similar to S100A10 and $\mathrm{Ca}^{2+}-\mathrm{S} 100 \mathrm{~A} 11$. However, binding of these two chimaeric proteins to phenyl Sepharose was less efficient than the wild type proteins. These observations suggest that the substitutions in site II of S100A11 act to stabilize the open conformation relative to the wild-type protein in the calcium-free state, altering the populations of closed and open state observed. In addition, NMR studies of S100A11 ${ }^{\mathrm{LI}}$ show evidence for structural differences between the chimera and both forms of S100A11, suggesting that the fully open state was not reached by the substitutions. This could be consistent with a shift in the equilibrium or an increase in the population for the open conformation. This concept has been well established for other EF-hand calciumbinding proteins such as calbindin $\mathrm{D}_{9 \mathrm{k}}$ and calmodulin where the conformations of these proteins has been altered by substitution [19]. NMR studies of the E104Q/E140Q mutant 
of the C-terminal domain of calmodulin by Akke and coworkers have elegantly shown an equilibrium exchange in the calcium-saturated state similar to the closed and open conformations of wild type calmodulin. Their findings provide evidence of the presence of a small but finite population of the open, calcium-bound protein, even though the closed conformation is favoured $[21-24,27]$. In apo-S100A $11^{\text {N66C,E75S }}$ and apoS100A $11^{\text {LII }}$ the substitutions likely act to stabilize and populate of the open state to a greater extent than in apo-S100A11. Such an effect implies either an increase in the free energy of the apo- closed form or a decrease in the free energy of the apo-closed state (Figure 6.1). However, more complex models can also be considered.

The increase in hydrophobicity in S100A $11^{\text {LII }}$ and S100A $11^{\text {N66C,E75S }}$ and the limited number of substitutions in each protein provides insights into residues that could have an important role in stabilizing the open states of S100A10 and $\mathrm{Ca}^{2+}-\mathrm{S} 100 \mathrm{~A} 11$. In S100A11, E75 occupies the last position (-Z) in the calcium-binding loop. As a result, substitution of this highly conserved residue in S100A $11^{\text {N66C,E75S }}$ and S100A1 $1^{\text {LII }}$ reduces their calcium binding abilities, similar to other EF-hand proteins. However, even in the presence of a functional $-\mathrm{Z}$ position, this residue contributes little to the conformational change towards the open state. Upon calcium binding in S100A11 the distance $(\sim 6.5 \AA)$ between E75 $(\mathrm{C} \beta)$ and the calcium ligand Q70 (CO) changes by less than $0.5 \AA$. Further, superposition of the C-terminal portion of site II and helix IV (K65-T79) from S100A10 which resides in the open state, reveals modest differences with the same region of the calcium-free forms (closed) for S100A2 (0.37 $\AA), \operatorname{S100A3}(0.63 \AA), \operatorname{S100A6}(0.63 \AA)$, S100B $(0.83 \AA)$ and S100A11 (0.96 $\AA)$. This indicates that despite being a critical residue 


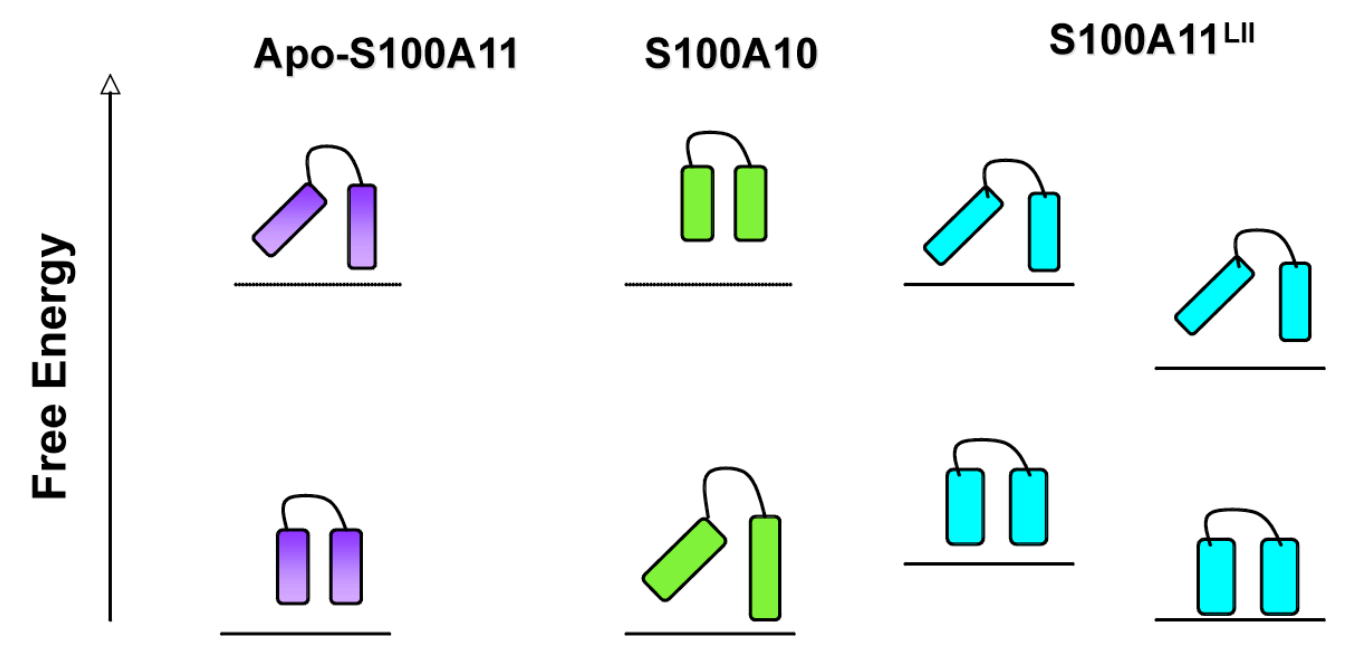

Figure 6.1 Possible models for free energy changes due to substitutions in loop II.

A shift in the equilibrium of the calcium-free S100A1 $1^{\mathrm{LII}}$ toward the open form, can be explained by an increase in the free energy of the apo/closed states or a decrease in free energy for the apo/open forms. 
for calcium binding in most other S100 proteins, E75 in S100A11 (S70 in S100A10) appears to have little influence on the open/closed conformations. This is in contrast with experiments for calmodulin and troponin- $\mathrm{C}$ that show substitution at the $-\mathrm{Z}$ coordinating position not only deters calcium binding but also favours a closed state [23, 24, 29, 30]. The structural comparison described for S100A10, shows this does not occur in the S100 proteins indicating the E75S replacement alone in S100A11 is not a strong determinant in favouring the open conformation in S100A10.

Unlike the structurally similar C-terminal portions of the site II calcium-binding loop in the closed (apo-S100A11) and open states (S100A10 and $\left.\mathrm{Ca}^{2+}-\mathrm{S} 100 \mathrm{~A} 11\right)$, the Nterminus of the loop (D59-D63) adopts very different conformations in these two states. In S100A10 the conformation is similar to that found in the calcium-bound forms of most S100 proteins. However in of calcium-free S100A2, S100A3, S100A11 and S100A6 it portion adopts an alternate conformation ("inverted", Figure 2.11), centered near the Y coordinating position, site of the N66C substitution used in both S100A1 $1^{\mathrm{N} 66 \mathrm{C}, \mathrm{E} 75 \mathrm{~S}}$ and S100A $11^{\text {LII }}$. Upon calcium binding this residue and the following $\mathrm{Y}+1$ residue reorient $\left(\Delta \phi \sim 30^{\circ}, \Delta \psi \sim 33^{\circ} ; \Delta \phi \sim 101^{\circ}, \Delta \psi \sim 80^{\circ}\right.$ respectively) leading to the large repositioning of helix III observed in $\mathrm{Ca}^{2+}-\mathrm{S} 100 \mathrm{~A} 11$ (or S100A10) and the exposed hydrophobic surface used for target binding. In the present studies, increased binding of S100A11 ${ }^{\mathrm{N} 66 \mathrm{C}, \mathrm{E} 75 \mathrm{~S}}$ to ANS and phenyl Sepharose were noted, suggesting that the N66C substitution might be a contributing factor to the increased hydrophobicity. In apoS100A11 the Y position occupied by N66 forms a salt bridge to K32 in site I. In contrast, in S100A10 the $\mathrm{Y}$ position (C61) is exposed and the analogous residue to $\mathrm{K} 32$ (K27) 
forms a salt bridge with D52 in helix III. This implies that substitution of N66 (N66C) in apo-S100A11 might favour the exposure of this residue and contribute to the stabilization of the open conformation as it does in S100A10. It is noteworthy that substitutions at N66 and E75 of S100A11 were sufficient for the protein to acquire an increased hydrophobic surface, but not sufficient for annexin A2 binding. Further, although five additional substitutions used in S100A11 ${ }^{\mathrm{LII}}$ enhanced annexin A2 binding in the calcium-free state, target binding was less efficient compared to either S100A10 or $\mathrm{Ca}^{2+}-\mathrm{S} 100 \mathrm{~A} 11$. These findings are similar to those for a modified S100P protein having three substitutions in site II that exhibited phenyl Sepharose binding in the absence of calcium and partial interaction with ezrin, one of its protein targets [26]. The S100P protein also contained a three-residue deletion in site I but it is unclear how this deletion contributes to the open conformation. In S100A11, a chimaeric protein possessing the site I sequence of S100A10 (S100A11 $\left.{ }^{\mathrm{LI}}\right)$ displays little increase in hydrophobicity in the absence of calcium and NMR experiments indicate that $\mathrm{S} 100 \mathrm{~A} 11^{\mathrm{LI}}$ is unable to adopt the open conformation even in the presence of calcium. When combined with the complete site II sequence from S100A10 (S100A11 ${ }^{\mathrm{LI}, \mathrm{LII})}$ the exposed hydrophobic surface is reduced compared to $\mathrm{S} 100 \mathrm{~A} 11^{\mathrm{LII}}$ alone indicating there is communication between the two calcium binding sites.

In summary the studies presented in this thesis have shown that substitutions in the C-terminal loop of S100A11 with those in S100A10 lead to changes in conformation and hydrophobicity that are consistent with a shift in the equilibrium towards the open state for apo-S100A11. This work suggests that the C-terminal loop important role for the 
unique active conformation of S100A10.

\subsection{Conclusions}

The work presented in this thesis has contributed valuable information to the field of the S100 proteins. The details of the regions in S100A10 most responsible for its open form and the differences between this protein and other calcium-activated S100 proteins have aided an understanding for the molecular basis of the unique conformation of S100A10. More importantly, it constitutes an important step towards re-engineering a calcium-free protein into a constitutively active form that would make identification and functional studies of in vivo targets possible.

\subsection{Future Directions}

The first set of chimaeric S100A11/S100A10 proteins showed that residues in site II is important contributors towards the open state in S100A10. An increase in the population of the open form was evident, however the open state was not fully reached suggesting that further refinement of this chimaeric protein is required. Inter-residue contact analysis showed that S100A10 is the only protein that has lysine residue at the $-\mathrm{y}$ position in the calcium binding loop (K65) which forms a unique hydrogen bond with a tyrosine residue in site I, (Y24, analogous toT29 in S100A11). Moreover, hydrophobic surface area experiments suggested that at least part of the shift in the equilibrium towards the open form takes place in S100A1 $1^{\mathrm{N} 66 \mathrm{C}, \mathrm{E} 75 \mathrm{~S}}$. Based on these results a good starting point for the next set of chimaeric proteins would incorporate a lysine residue at position $-\mathrm{y}$ as in S100A10 and the interacting tyrosine residue in site I: $\mathrm{S} 100 \mathrm{~A} 11^{\mathrm{T} 29 \mathrm{~K} / \mathrm{N} 66 \mathrm{C}, \mathrm{Q} 70 \mathrm{~K}, \mathrm{E} 75 \mathrm{~S}}$. It would also be necessary to investigate which deletions 
present in site I allow the formation of the hydrogen bond between K65/Y24. The results in these studies showed that S100A1 $1^{\mathrm{LI}}$ has decreased calcium affinity and is no longer is able to adopt the open conformation in the presence of calcium. Interesting studies would include a set of substitutions in site I to identify which residues influences the open conformation of S100A11 $1^{\mathrm{N} 66 \mathrm{C}, \mathrm{E} 75 \mathrm{~S}}$, as postulated in the work reported for S100P [26]. Once an apo-S100A11 protein reaching the open conformation in the absence of calcium has been designed it could be used in yeast two hybrid experiments to determine in vivo target protein interactions. It is envisaged that similar approaches could be used to identify in vivo protein targets for other members of the S100 protein family. 


\subsection{References}

1 Ikura, M., Clore, G. M., Gronenborn, A. M., Zhu, G., Klee, C. B. and Bax, A. (1992) Solution structure of a calmodulin-target peptide complex by multidimensional NMR. Science. 256, 632-638

2 Smith, S. P. and Shaw, G. S. (1998) A novel calcium-sensitive switch revealed by the structure of human S100B in the calcium-bound form. Structure. 6, 211-222

3 Herzberg, O., Moult, J. and James, M. N. G. (1986) A model for the $\mathrm{Ca}^{2+}$-induced conformational transition of troponin C. J. Biol. Chem. 261, 2638-2644

4 Santamaria-Kisiel, L., Rintala-Dempsey, A. C. and Shaw, G. S. (2006) Calciumdependent and -independent interactions of the S100 protein family. Biochem J. 396, 201-214

5 Wilder, P. T., Rustandi, R. R., Drohat, A. C. and Weber, D. J. (1998) S100B( $\beta \beta$ ) inhibits the protein kinase $\mathrm{C}$-dependent phosphorylation of a peptide derived from p53 in a $\mathrm{Ca}^{2+}$-dependent manner. Protein Sci. 7, 794-798

6 Odink, K., Cerletti, N., Bruggen, J., Clerc, R. G., Tarcsay, L., Zwadlo, G., Gerhards, G., Schlegel, R. and Sorg, C. (1987) Two calcium-binding proteins in infiltrate macrophages of rheumatoid arthritis. Nature. 330, 80-82

7 Van Eldik, L. J. and Griffin, W. S. T. (1994) S100b expression in Alzheimer's disease: Relation to neuropathology in brain regions. Biochim. Biophys. Acta. 1223, 398-403

8 Bianchi, R., Giambanco, I., Arcuri, C. and Donato, R. (2003) Subcellular localization of S100A11 (S100C) in LLC-PK1 renal cells: calcium- and protein kinase C-dependent association of S100A11 with S100B and vimentin intermediate filaments. Microsc Res Tech. 60, 639-651

9 Rintala-Dempsey, A. C., Santamaria-Kisiel, L., Liao, Y., Lajoie, G. and Shaw, G. S. (2006) Insights into S100 target specificity examined by a new interaction between S100A11 and annexin A2. Biochemistry. 45, 14695-14705

10 Seemann, J., Weber, K. and Gerke, V. (1996) Structural requirements for annexin IS100C complex-formation. Biochem. J. 319, 123-129

11 Seemann, J., Weber, K. and Gerke, V. (1997) Annexin I targets S100C to early endosomes. FEBS Lett. 413, 185-190

12 Dempsey, A. C., Walsh, M. P. and Shaw, G. S. (2003) Unmasking the annexin I interaction from the structure of Apo-S100A11. Structure (Camb). 11, 887-897

13 Rety, S., D., O., Arie, J.-P., Tabaries, S., Seeman, J., Russo-Marie, F., Gerke, V. and Lewit-Bentley, A. (2000) Structural basis of the $\mathrm{Ca}^{2+}$ - dependent association between S100C (S100A11) and its target, the N-terminal part of annexin I. Structure. 8, 175-184

14 Rety, S., Sopkova, J., Renouard, M., Osterloh, D., Gerke, V., Tabaries, S., RussoMarie, F. and Lewit-Bentley, A. (1999) The crystal structure of a complex of p11 with the annexin II N-terminal peptide. Nat Struct Biol. 6, 89-95

15 Kuroki, R., Taniyama, Y., Seko, C., Nakamura, H., Kikuchi, M. and Ikehara, M. (1989) Design and creation of a Ca2+ binding site in human lysozyme to enhance structural stability. Proc Natl Acad Sci U S A. 86, 6903-6907 
16 Yang, W., Jones, L. M., Isley, L., Ye, Y., Lee, H. W., Wilkins, A., Liu, Z. R., Hellinga, H. W., Malchow, R., Ghazi, M. and Yang, J. J. (2003) Rational design of a calcium-binding protein. J Am Chem Soc. 125, 6165-6171

17 Balakrishnan, S. and Zondlo, N. J. (2006) Design of a protein kinase-inducible domain. J Am Chem Soc. 128, 5590-5591

18 Linse, S., Brodin, P., Drakenberg, T., Thulin, E., Sellers, P., Elmden, K., Grundstrom, T. and Forsen, S. (1987) Structure-function relationships in EF-hand $\mathrm{Ca} 2+-$ binding proteins. Protein engineering and biophysical studies of calbindin D9k. Biochemistry. 26, 6723-6735

19 Linse, S., Bylsma, N. R., Drakenberg, T., Sellers, P., Forsen, S., Thulin, E., Svensson, L. A., Zajtzeva, I., Zajtsev, V. and Marek, J. (1994) A calbindin D9k mutant with reduced calcium affinity and enhanced cooperativity. Metal ion binding, stability, and structural studies. Biochemistry. 33, 12478-12486

20 Marsden, B. J., Hodges, R. S. and Sykes, B. D. (1989) A ${ }^{1}$ H NMR determination of the solution conformation of a synthetic peptide analogue of calcium-binding site III of rabbit skeletal troponin C. Biochemistry. 28, 8839-8847

21 Evenas, J., Forsen, S., Malmendal, A. and Akke, M. (1999) Backbone dynamics and energetics of a calmodulin domain mutant exchanging between closed and open conformations. J Mol Biol. 289, 603-617

22 Evenas, J., Malmendal, A. and Akke, M. (2001) Dynamics of the transition between open and closed conformations in a calmodulin C-terminal domain mutant. Structure. 9, 185-195

23 Evenas, J., Malmendal, A., Thulin, E., Carlstrom, G. and Forsen, S. (1998) Ca2+ binding and conformational changes in a calmodulin domain. Biochemistry. 37, 13744-13754

24 Evenas, J., Thulin, E., Malmendal, A., Forsen, S. and Carlstrom, G. (1997) NMR studies of the E140Q mutant of the carboxy-terminal domain of calmodulin reveal global conformational exchange in the $\mathrm{Ca} 2+$-saturated state. Biochemistry. 36, 34483457

25 Bunick, C. G., Nelson, M. R., Mangahas, S., Hunter, M. J., Sheehan, J. H., Mizoue, L. S., Bunick, G. J. and Chazin, W. J. (2004) Designing sequence to control protein function in an EF-hand protein. J Am Chem Soc. 126, 5990-5998

26 Austermann, J., Nazmi, A. R., Heil, A., Fritz, G., Kolinski, M., Filipek, S. and Gerke, V. (2009) Generation and characterization of a novel, permanently active S100P mutant. Biochim Biophys Acta. 1793, 1078-1085

27 Lundstrom, P. and Akke, M. (2004) Quantitative analysis of conformational exchange contributions to $1 \mathrm{H}-15 \mathrm{~N}$ multiple-quantum relaxation using fielddependent measurements. Time scale and structural characterization of exchange in a calmodulin C-terminal domain mutant. J Am Chem Soc. 126, 928-935

28 Ababou, A., Shenvi, R. A. and Desjarlais, J. R. (2001) Long-range effects on calcium binding and conformational change in the $\mathrm{N}$-domain of calmodulin. Biochemistry. 40, 12719-12726

29 McKay, R. T., Saltibus, L. F., Li, M. X. and Sykes, B. D. (2000) Energetics of the induced structural change in a $\mathrm{Ca} 2+$ regulatory protein: $\mathrm{Ca} 2+$ and troponin I peptide 
binding to the E41A mutant of the N-domain of skeletal troponin C. Biochemistry. 39, 12731-12738

30 Gagne, S. M., Li, M. X. and Sykes, B. D. (1997) Mechanism of direct coupling between binding and induced structural change in regulatory calcium binding proteins. Biochemistry. 36, 4386-4392 


\section{Appendix A}

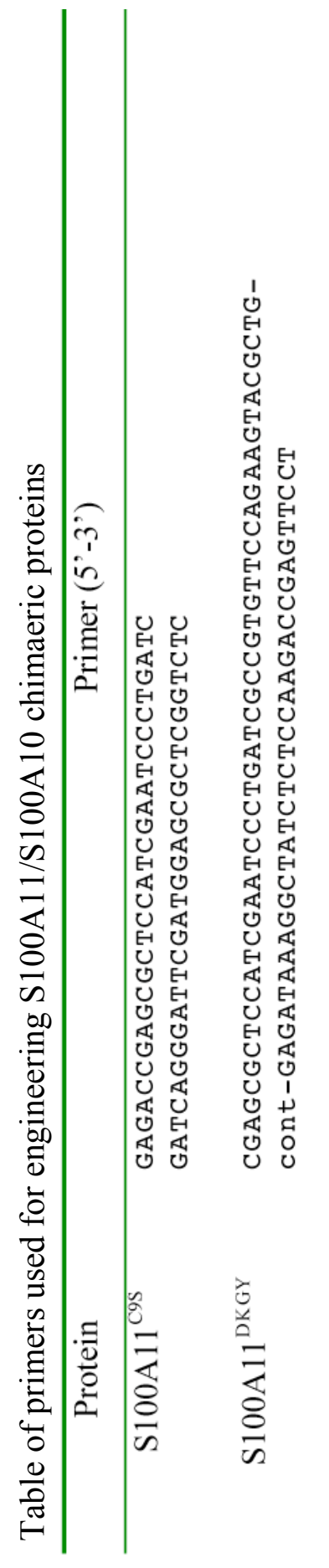

U

U

遮

U U

넙

鼠

텅

才ֶ㑔

要议䀝质

更电

U质芯

䊆唨

번에

ک 0

OU U

버업

论

U 近芯

y 00

或

还更记

प ब

버업

乐记

试舁订

兒讧芯舅

번

U瓜远

近出乐

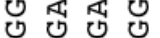

법법

瓜昏

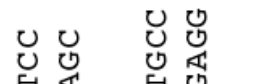

旡㫐

串 U

प ब

才 夰

过

य ठ 己 ठ ग

U

瓜

ह

呢百记

保

《

证

近

采

Uु

U D

论

O O

记

प

บ 更

U

氒

법

o $\mathrm{H}$

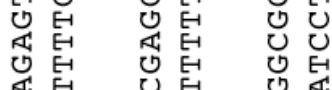

证 0

开

讨

促

U

Oํㅓㅁ

䒴芯

芯记芯

近

式 质

번 U U

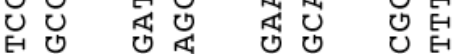

后芯讨

尫记

Uુ

オ̛̃

Уण

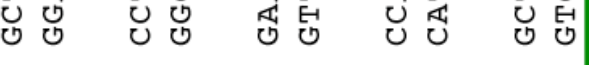




\section{Appendix B}

Chimaeric S100A11/S100A1 proteins engineered

\begin{tabular}{|c|c|c|}
\hline Chimaeric Protein & Modified Sequence & Schematic \\
\hline $\mathrm{S} 100 \mathrm{~A} 11^{\mathrm{LI}}$ & ${ }^{21}$ AGDKGYLTKED $^{34}$ & \\
\hline $\mathrm{S} 100 \mathrm{~A} 11^{\mathrm{N} 66 \mathrm{C}, \mathrm{E} 75 \mathrm{~S}}$ & ${ }^{65} \mathrm{LNSDGQLDFQE}^{75}$ & \\
\hline S100A11 LII & ${ }^{65} \mathrm{QCRDGKVGFQS}^{75}$ & \\
\hline S100A1 $11^{\text {LI, LII }}$ & $\begin{array}{l}{ }^{21} \mathrm{AGDKGYLTKED}^{34} \\
{ }^{65} \mathrm{QCRDGKVGFQS}^{75}\end{array}$ & \\
\hline $\mathrm{S} 100 \mathrm{~A} 11^{\mathrm{H} 2, \mathrm{H} 3}$ & $\begin{array}{l}{ }^{35} \text { LRVLMEKE }^{42}, \\
{ }^{53} \text { PLAVDKIMKDL }^{63}\end{array}$ & \\
\hline $\mathrm{S} 100 \mathrm{~A} 11^{\mathrm{H} 2-\mathrm{L}-\mathrm{H} 3}$ & $\begin{array}{l}{ }^{35} \text { LRVLMEKEFPGFLENQKD } \\
\text { PLAVDKIMKDL }\end{array}$ & \\
\hline $\mathrm{S} 100 \mathrm{~A} 11^{\mathrm{H} 3, \mathrm{H} 4}$ & 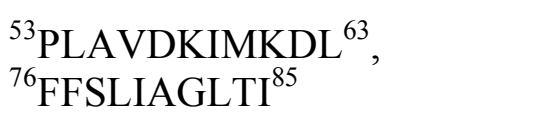 & \\
\hline
\end{tabular}




\section{Appendix C}

Differences in surface area exposure ${ }^{\mathrm{a}}$ for side chain residues in S100A10 and S100A11

\begin{tabular}{|c|c|c|c|c|}
\hline $\begin{array}{l}\text { Residue } \\
\text { Number }\end{array}$ & $\begin{array}{c}\text { Residue } \\
\text { (rabbit } \\
\text { S100A11) }\end{array}$ & $\begin{array}{c}\Delta \text { ASA } \\
\text { S100A10 vs } \\
\text { Apo-S100A11 }\end{array}$ & $\begin{array}{c}\Delta \text { ASA } \\
\mathrm{Ca}^{2+}-\mathrm{S} 100 \mathrm{~A} 11 \mathrm{vs} \\
\mathrm{Apo}-\mathrm{S} 100 \mathrm{~A} 11\end{array}$ & $\begin{array}{c}\Delta \text { ASA } \\
\text { S100A10 vs } \mathrm{Ca}^{2+}- \\
\text { S100A11 }\end{array}$ \\
\hline 1 & SER & & & \\
\hline 2 & ARG & & & \\
\hline 3 & PRO & 0.37 & 0.41 & -0.04 \\
\hline 4 & THR & -0.21 & -0.03 & -0.18 \\
\hline 5 & GLU & -0.29 & -0.45 & 0.16 \\
\hline 6 & THR & -0.01 & -0.01 & 0.00 \\
\hline 7 & GLU & 0.02 & -0.03 & 0.05 \\
\hline 8 & ARG & -0.27 & -0.51 & 0.24 \\
\hline 9 & CYS & 0.01 & -0.01 & 0.02 \\
\hline 10 & ILE & 0.05 & 0.06 & -0.01 \\
\hline 11 & GLU & -0.06 & 0.16 & -0.22 \\
\hline 12 & SER & -0.02 & -0.18 & 0.16 \\
\hline 13 & LEU & -0.01 & 0.01 & -0.02 \\
\hline 14 & ILE & 0.00 & 0.06 & -0.06 \\
\hline 15 & ALA & -0.02 & 0.15 & -0.17 \\
\hline 16 & VAL & -0.02 & -0.01 & -0.01 \\
\hline 17 & PHE & -0.01 & -0.02 & 0.01 \\
\hline 18 & GLN & -0.19 & -0.18 & -0.01 \\
\hline 19 & LYS & -0.05 & -0.06 & 0.01 \\
\hline 20 & TYR & -0.17 & -0.26 & 0.09 \\
\hline 21 & ALA & -0.01 & -0.01 & 0.00 \\
\hline 22 & GLY & 0.12 & -0.41 & 0.53 \\
\hline 23 & LYS & 0.15 & -0.43 & 0.58 \\
\hline 24 & ASP & ---- & -0.44 & \\
\hline 25 & GLY & ---- & 0.05 & \\
\hline 26 & HIS & -0.48 & -0.52 & 0.04 \\
\hline 27 & SER & -0.19 & -0.15 & -0.04 \\
\hline 28 & VAL & ---- & -0.10 & \\
\hline 29 & THR & 0.21 & 0.21 & 0.00 \\
\hline 30 & LEU & -0.02 & -0.02 & 0.00 \\
\hline 31 & SER & 0.00 & -0.01 & 0.01 \\
\hline 32 & LYS & -0.31 & -0.23 & -0.08 \\
\hline 33 & THR & 0.05 & 0.15 & -0.10 \\
\hline 34 & GLU & -0.22 & -0.44 & 0.22 \\
\hline 35 & PHE & 0.00 & 0.00 & 0.00 \\
\hline 36 & LEU & -0.06 & 0.08 & -0.14 \\
\hline 37 & SER & 0.13 & 0.01 & 0.12 \\
\hline 38 & PHE & 0.00 & 0.00 & 0.00 \\
\hline 39 & MET & 0.00 & 0.00 & 0.00 \\
\hline 40 & ASN & -0.05 & -0.11 & 0.06 \\
\hline 41 & THR & 0.09 & -0.07 & 0.16 \\
\hline
\end{tabular}




\section{Appendix C}

Differences in surface area exposure ${ }^{\mathrm{a}}$ for side chain residues in S100A10 and S100A11

\begin{tabular}{|c|c|c|c|c|}
\hline $\begin{array}{l}\text { Residue } \\
\text { Number }\end{array}$ & $\begin{array}{c}\text { Residue } \\
\text { (rabbit } \\
\text { S100A11) } \\
\end{array}$ & $\begin{array}{c}\Delta \text { ASA } \\
\text { S100A10 vs } \\
\text { Apo-S100A11 }\end{array}$ & $\begin{array}{c}\Delta \mathbf{A S A} \\
\mathrm{Ca}^{2+}-\mathrm{S} 100 \mathrm{~A} 11 \mathrm{vs} \\
\text { Apo-S100A11 }\end{array}$ & $\begin{array}{c}\Delta \text { ASA } \\
\text { S100A10 vs } \mathrm{Ca}^{2+}- \\
\text { S100A11 }\end{array}$ \\
\hline 42 & GLU & -0.36 & -0.33 & -0.03 \\
\hline 43 & LEU & 0.04 & 0.04 & 0.00 \\
\hline 44 & ALA & 0.22 & 0.05 & 0.17 \\
\hline 45 & ALA & 0.36 & 0.30 & 0.06 \\
\hline 46 & PHE & 0.30 & 0.24 & 0.06 \\
\hline 47 & THR & 0.02 & 0.05 & -0.03 \\
\hline 48 & LYS & -0.08 & 0.10 & -0.18 \\
\hline 49 & ASN & -0.08 & -0.07 & -0.01 \\
\hline 50 & GLN & 0.05 & -0.16 & 0.21 \\
\hline 51 & LYS & -0.01 & 0.04 & -0.05 \\
\hline 52 & ASP & 0.01 & 0.26 & -0.25 \\
\hline 53 & PRO & 0.28 & 0.27 & 0.01 \\
\hline 54 & GLY & -0.10 & -0.35 & 0.25 \\
\hline 55 & VAL & 0.11 & 0.24 & -0.13 \\
\hline 56 & LEU & -0.21 & -0.15 & -0.06 \\
\hline 57 & ASP & -0.18 & -0.10 & -0.08 \\
\hline 58 & ARG & 0.28 & 0.39 & -0.11 \\
\hline 59 & MET & 0.20 & 0.18 & 0.02 \\
\hline 60 & MET & -0.10 & -0.16 & 0.06 \\
\hline 61 & LYS & 0.06 & -0.10 & 0.16 \\
\hline 62 & LYS & 0.13 & 0.29 & -0.16 \\
\hline 63 & LEU & -0.14 & -0.09 & -0.05 \\
\hline 64 & ASP & -0.91 & -0.98 & 0.07 \\
\hline 65 & LEU & 0.61 & 0.39 & 0.22 \\
\hline 66 & ASN & -0.15 & -0.31 & 0.16 \\
\hline 67 & SER & -0.12 & -0.29 & 0.17 \\
\hline 68 & ASP & -0.22 & -0.31 & 0.09 \\
\hline 69 & GLY & -0.18 & -0.25 & 0.07 \\
\hline 70 & GLN & 0.00 & -0.20 & 0.20 \\
\hline 71 & LEU & -0.01 & -0.01 & 0.00 \\
\hline 72 & ASP & 0.27 & 0.07 & 0.20 \\
\hline 73 & PHE & -0.13 & -0.16 & 0.03 \\
\hline 74 & GLN & 0.56 & 0.36 & 0.20 \\
\hline 75 & GLU & 0.28 & 0.01 & 0.27 \\
\hline 76 & PHE & 0.00 & 0.00 & 0.00 \\
\hline 77 & LEU & -002 & 0.03 & -0.05 \\
\hline 78 & ASN & 0.40 & 0.24 & 0.16 \\
\hline 79 & LEU & 0.11 & 0.03 & 0.08 \\
\hline 80 & ILE & 0.00 & 0.02 & -0.02 \\
\hline 81 & GLY & 0.20 & 0.27 & -0.07 \\
\hline
\end{tabular}




\section{Appendix C}

Differences in surface area exposure ${ }^{a}$ for side chain residues in S100A10 and S100A11

\begin{tabular}{ccrrr}
\hline $\begin{array}{l}\text { Residue } \\
\text { Number }\end{array}$ & $\begin{array}{c}\text { Residue } \\
\text { (rabbit } \\
\text { S100A11) }\end{array}$ & $\begin{array}{c}\Delta \text { ASA }^{\mathrm{a}} \\
\text { S100A10 vs } \\
\text { Apo-S100A11 }\end{array}$ & $\begin{array}{c}\Delta \text { ASA }^{\mathrm{a}} \\
\text { Ca }^{2+}-\text { S100A11 vs } \\
\text { Apo-S100A11 }\end{array}$ & $\begin{array}{r}\Delta \text { ASA }^{\mathrm{a}} \\
\text { S100A10 vs Ca }{ }^{2+} \\
\text { S100A11 }\end{array}$ \\
\hline 82 & GLY & 0.64 & 0.57 & 0.07 \\
83 & LEU & 0.15 & 0.19 & -0.04 \\
84 & ALA & -0.02 & -0.02 & 0.00 \\
85 & VAL & 0.23 & 0.28 & -0.05 \\
86 & ALA & 0.49 & 0.49 & 0.00 \\
87 & CYS & 0.39 & 0.39 & 0.00 \\
88 & HIS & -0.19 & -0.17 & -0.02 \\
89 & GLU & 0.29 & 0.11 & 0.18 \\
90 & SER & 0.59 & 0.69 & -0.10 \\
91 & PHE & -0.29 & -0.02 & -0.27 \\
92 & VAL & 0.09 & -0.15 & 0.24 \\
93 & LYS & 0.02 & 0.15 & -0.13 \\
94 & ALA & 0.02 & 0.11 & -0.09 \\
95 & ALA & 0.41 & 0.23 & 0.18 \\
96 & PRO & 0.15 & 0.39 & 0.31 \\
\hline
\end{tabular}

${ }^{a}$ Difference in fractional accessible surface area $(\triangle A S A)$ for the side chains of $S 100 A 10$ and apo-S100A $1^{\mathrm{b}}, \mathrm{Ca}^{2+}-\mathrm{bound}$ and free forms of S100A11 $\left(\mathrm{Ca}^{2+}-\mathrm{S} 100 \mathrm{~A} 11 \mathrm{vs}\right.$ apo-S100A11) ${ }^{\mathrm{C}}$, and S100A10 and $\mathrm{Ca}^{2+}-\mathrm{S} 100 \mathrm{~A} 11$ (S100A10 vs Ca ${ }^{2+}$ S100A11). 


\section{Appendix D}

Summary of $\alpha$-carbon distances in apo-S100A11, $\mathrm{Ca}^{2+}$ loaded S100A11 and S100A11

\begin{tabular}{|c|c|c|c|c|c|c|}
\hline \multirow{2}{*}{$\begin{array}{l}\text { Helices } \\
\mathrm{H} 1 / \mathrm{H} 2\end{array}$} & \multicolumn{2}{|c|}{ Apo-S100A11 } & \multicolumn{2}{|c|}{$\mathrm{Ca}^{2+}-\mathrm{S} 100 \mathrm{~A} 11$} & \multicolumn{2}{|c|}{ S100A10 } \\
\hline & Y20/F35 & 7.20 & H20/F37 & 7.62 & F18/L30 & 7.91 \\
\hline & Y20/F38 & 7.30 & $\mathrm{H} 22 / \mathrm{F} 40$ & 6.41 & F18/L33 & 6.48 \\
\hline & & & $\mathrm{H} 22 / \mathrm{I} 39$ & 7.92 & F18/V32 & 7.86 \\
\hline $\mathrm{H} 1 / \mathrm{H} 3$ & - & - & - & - & - & - \\
\hline \multirow[t]{5}{*}{$\mathrm{H} 1 / \mathrm{H} 4$} & I13/L77 & 6.45 & & & & \\
\hline & I14/L77 & 7.44 & & & & \\
\hline & I17/L77 & 7.98 & & & & \\
\hline & V16/F76 & 7.97 & & & & \\
\hline & F17/F76 & 5.89 & F19/F78 & 7.61 & F15/F71 & 7.64 \\
\hline \multirow[t]{3}{*}{$\mathrm{H} 2 / \mathrm{H} 3$} & - & - & F37/M63 & 7.95 & L30/M55 & 7.76 \\
\hline & & & L38/L58 & 7.38 & R31/V51 & 6.17 \\
\hline & & & & & L30/V51 & 7.57 \\
\hline $\mathrm{H} 2 / \mathrm{H} 4$ & - & - & - & - & - & - \\
\hline \multirow[t]{16}{*}{$\mathrm{H} 3 / \mathrm{H} 4$} & $59 \mathrm{M} / \mathrm{L} 79$ & 5.74 & & & & \\
\hline & $55 \mathrm{~V} / 83 \mathrm{~L}$ & 5.77 & & & & \\
\hline & $55 \mathrm{~V} / 86 \mathrm{~A}$ & 5.8 & & & & \\
\hline & M59/L83 & 7.30 & & & & \\
\hline & M59/G82 & 6.76 & & & & \\
\hline & M59/N78 & 7.98 & & & & \\
\hline & M60/L79 & 6.14 & & & & \\
\hline & V55/C87 & 7.09 & & & & \\
\hline & L63/E75 & 6.58 & & & & \\
\hline & L63/N78 & 7.38 & L65/N80 & 7.32 & & \\
\hline & L63/L79 & 6.78 & & & L58/L74 & 7.61 \\
\hline & L56/L83 & 6.39 & & & & \\
\hline & V55/G82 & 7.98 & & & & \\
\hline & L56/L79 & 7.68 & & & & \\
\hline & & & L65/F78 & 7.50 & L58/F71 & 7.74 \\
\hline & & & L65/L79 & 7.05 & & \\
\hline
\end{tabular}

${ }^{a}$ Helices are defined as follows: Helix 1: 5-20 (apo-S100A11), 7-22 ( $\left.\mathrm{Ca}^{2+}-\mathrm{S} 100 \mathrm{~A} 11\right), 3-18$ (S100A10); Helix II 35-42 (apoS100A11), 37-44 (Ca $\left.{ }^{2+}-\mathrm{S} 100 \mathrm{~A} 11\right), 30-37$ (S100A10); Helix III 53-63 (apo-S100A11), 55-65 (Ca $\left.{ }^{2+}-\mathrm{S} 100 \mathrm{~A} 11\right), 48-58$ (S100A10); Helix IV 76-85 (apo-S100A11), 78-87 (Ca $\left.{ }^{2+}-\mathrm{S} 100 \mathrm{~A} 11\right), 71-80$ (S100A10).

${ }^{\mathrm{b}} \mathrm{PDB}$ code: $1 \mathrm{NSH}$

${ }^{\mathrm{C}}$ PDB code: $1 \mathrm{QLS}$

${ }^{\mathrm{d}}$ PDB code: 1BT6 


\section{CURRICULUM VITAE}

\section{LILIANA SANTAMARIA-KISIEL}

Department of Biochemistry

Faculty of Medicine and Dentistry

\section{Education}

PhD. Degree in Biochemistry

2011

Thesis: "Chimaeric S100A11/S100A10 proteins"

University of Western Ontario. London, ON

Bachelor of Science Honours Chemistry

Thesis: "Characterization of preliminary stages prior to encystations in Giardia lamblia"

(Meritorious Mention Award. Top 2\% students).

National University of Colombia. Bogotá. Colombia

\section{Research Experience}

\section{Research and Development Technician}

Biomolecular Interactions and Conformations Facility

University of Western Ontario. London, ON

Research Technician

Department of Microbiology and Immunology

University of Western Ontario. London, ON

Laboratory Attendant

London Public Health Laboratory. London, ON

Research Student (Honor's Thesis Award)

Full time research. $>40 \mathrm{~h} /$ week for 16 months

National University of Colombia. Bogotá, Colombia
June 2010-May 2011

Supervisor: Dr. Stan Dunn

2002-2003

Supervisor: Dr. Miguel Valvano

Supervisor: Dr. Abdul Chagla

2002

2000-2001

Supervisor: Dr. Moises Wasserman

\section{Honors and Awards}

Western Graduate Research Scholarship (WGRS)

Ontario Graduate Scholarship

2007-2008

Western Graduate Research Scholarship (WGRS)

2007-2008

Schulich Scholarship for Medicine Research

Graduate Thesis Research Award

2007

Ontario Graduate Scholarship in Science and Technology

2006-2007

Western Graduate Research Scholarship (WGRS)

2006-2007

Departmental Nominee for Nellie L. Farthing Memorial Fellowship

(Recognition Excellence in Research for Senior PhD. Students in Medical Sciences) 


\section{Honors and Awards Cont'd}

Ontario Graduate Scholarship in Science and Technology

2004-2005

Special University Scholarship (SUS). University of Western Ontario

2003-2004

Meritorious Mention Bachelor's thesis award. National University of Colombia

(Recognition novelty and excellence in research development. Top 2\% students)

2000

Tuition Scholarship. "Hijas de Cristo Rey" High School. Bogotá, Colombia

(Private high school full tuition. Top 5\% students)

$1991-1994$

\section{Publications}

\section{Refereed:}

1. Santamaria-Kisiel, L., and Shaw, G. (2006) Calcium dependent and independent interaction of the S100 protein family. Biochem. J. 396 (201-214).

2. Dempsey-Rintala, A.C., Santamaria-Kisiel, L., Liao Y., Lajoie, G. and Shaw, G Insights into S100 Target Specificity Examined by a New Interaction between S100A11 and Annexin A2. Biochemistry (2006) 49 14695-14705.

3. Santamaria-Kisiel, L., Rintala-Dempsey, A., and Shaw, G. (2011) Identification of regions responsible for the open conformation of S100A10 using chimaeric S100A11-S100A10

4. proteins. Biochem. J. 434 (37-48).

5. Santamaria-Kisiel, L., and Shaw, G. Protein-Protein interaction of the S100 proteins. A Thermodynamic approach. Manuscript in preparation.

\section{Non-Refereed:}

1. Santamaria-Kisiel, L., Bachelor's Honours Thesis (150 pages). (requirement to obtain a Bachelor's degree in Colombia). (2000-2001). "Characterization of preliminary stages prior to encystations in Giardia lamblia".

2. Santamaria, L., Fierro, R., and Calle, M.L. Polymers in dentistry. From Theory to Practice. Laboratory Manual Guide. (1999) School of Medicine. National University of Colombia.

\section{Conference Presentations}

1. Santamaria-Kisiel, L. and Shaw, G. Towards the design of a functionally active apo-S100 protein. (2007). 15 $5^{\text {th }}$ International Symposium on Calcium and Calcium Binding Proteins in Health and Disease. La Palma, Spain.

2. Santamaria-Kisiel, L. and Shaw, G Structural insights into the permanently open form of S100A10 (2006). The $20^{\text {th }}$ Symposium of the Protein Society. San Diego, California.

3. Santamaria-Kisiel, L. and Shaw, G. Engineering of "calcium-ready" S100A11. (2005). 14 International Symposium on Calcium and Calcium Binding Proteins in Health and Disease. Banff, Alberta, Canada 


\section{Poster Presentations}

1. Santamaria-Kisiel, L. and Shaw, G. Understanding the open form of S100A10. (2006). Margaret P. Moffat Graduate Research Day. University of Western Ontario.

2. Santamaria-Kisiel, L. and Shaw, G. Understanding the permanently open form of S100A10. (2006). Dept. of Biochemistry. University of Western Ontario.

3. Santamaria-Kisiel, L. and Shaw, G. Engineering of "calcium-ready" S100A11. (2005). Margaret P. Moffat Graduate Research Day. University of Western Ontario.

4. Santamaria, $\mathrm{L}$. Characterization of preliminary stages prior to encystations in Giardia lamblia (2000). Dept. of Chemistry. National University of Colombia.

\section{Oral Presentations}

1. Santamaria-Kisiel, L. and Shaw, G. Protein-Protein interaction of the S100 proteins. A Thermodynamic approach (2007). Department of Biochemistry. University of Western Ontario.

2. Santamaria-Kisiel, L. and Shaw, G. Towards the design of a functionally active apo-S100 protein (2006). Department of Biochemistry. University of Western Ontario.

3. Santamaria-Kisiel, L. and Shaw, G. Engineering of "calcium-ready" S100A11. (2005). Department of Biochemistry. University of Western Ontario.

4. Santamaria L. Characterization of preliminary stages prior to encystations in Giardia lamblia. (2001). Department of Chemistry, National University of Colombia.

\section{Teaching Assistantships}

Protein structure, analysis and design. Biochemistry 440a 2006

Faculty of Medicine and Dentistry. The University of Western Ontario. 5h/week

Protein structure, analysis and design. Biochemistry 440a

Highly evaluated for a group of 29 students.

Faculty of Medicine and Dentistry. The University of Western Ontario. 5h/week

Second year General Chemistry Course

Faculty of Medicine. University of "El Bosque". Colombia-Colombia

Fourth year Polymers Course

$1999-2000$

Faculty of Dentistry. National University of Colombia. Colombia-Colombia

\section{Committees and Activities}

Mentorship Grade 11 student

Faculty of Medicine and Dentistry. Dept of Biochemistry UWO.

The Protein Society Member

Faculty of Graduate Studies Showcase

Faculty of Medicine and Dentistry. Dept of Biochemistry UWO.

Area, Safety and Equipment Committee member

2006-2007

Faculty of Medicine and Dentistry. Dept of Biochemistry UWO.

Nominating Committee member

2005-2006

Faculty of Medicine and Dentistry. Dept of Biochemistry UWO.

Social Committee member.

2004-2005

Faculty of Medicine and Dentistry. Dept of Biochemistry UWO.

Secretary Chemistry Students Organization.

1998-1999

National University of Colombia 
